\title{
Synthesis of Complex Boron-Nitrogen Heterocycles Comprising Borylated Triazenes and Tetrazenes Under Mild Conditions.
}

Dominic Prieschl,, ${ }^{+1,2}$ Guillaume Bélanger-Chabot, ${ }^{+, 1,2}$ Xueying Guo, ${ }^{+, 3}$ Maximilian Dietz, ${ }^{1,2}$ Marcel Müller, ${ }^{1,2}$ Ivo Krummenacher, ${ }^{1,2}$ Zhenyang Lin, ${ }^{*, 3}$ Holger Braunschweig, ${ }^{*, 1,2}$

\footnotetext{
${ }^{+}$These authors contributed equally to this work

${ }^{1}$ Institute for Inorganic Chemistry, Julius-Maximilians-Universität Würzburg, Am Hubland, 97074 Würzburg, Germany

${ }^{2}$ Institute for Sustainable Chemistry \& Catalysis with Boron, Julius-Maximilians-Universität Würzburg, Am Hubland, 97074 Würzburg, Germany

${ }^{3}$ Department of Chemistry, The Hong Kong University of Science and Technology, Clear Water Bay, Kowloon, Hong Kong, China
}

\section{$\underline{\text { Table of contents }}$}

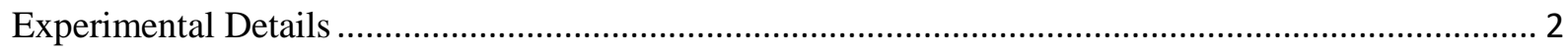

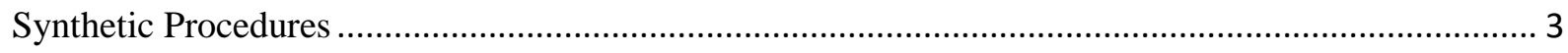

NMR Spectra

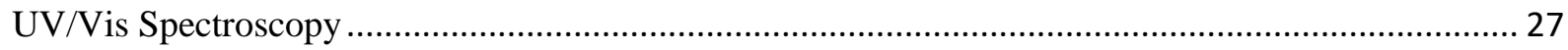

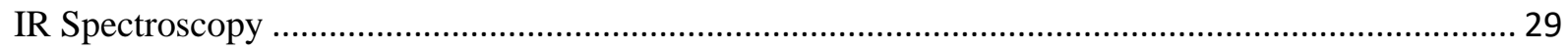

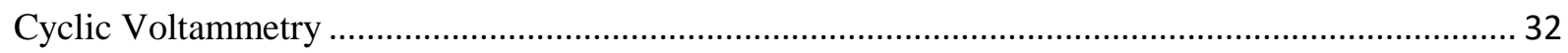

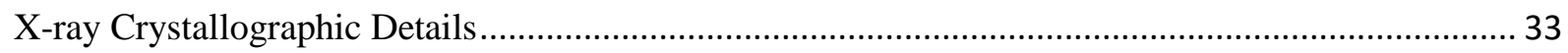

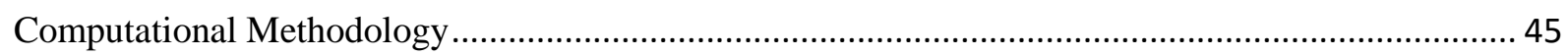

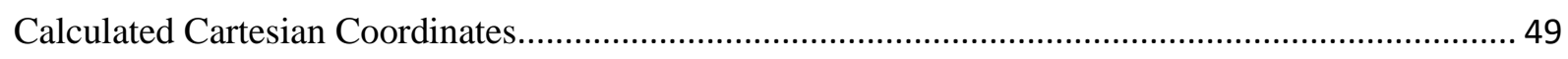

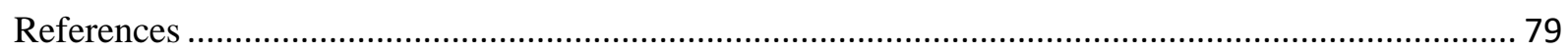




\section{Experimental Details}

\section{General Information}

All manipulations were performed either under an atmosphere of dry argon or in vacuo using standard Schlenk line or glovebox techniques. Deuterated solvents were dried over molecular sieves and degassed by three freeze-pump-thaw cycles prior to use. All other solvents were distilled and degassed from appropriate drying agents. Solvents (both deuterated and non-deuterated) were stored under argon over activated 4 A molecular sieves. NMR spectra were acquired on a Bruker Avance 500 NMR spectrometer $\left({ }^{1} \mathrm{H}: 500.1 \mathrm{MHz},{ }^{11} \mathrm{~B}: 160.5 \mathrm{MHz},{ }^{13} \mathrm{C}\left\{{ }^{1} \mathrm{H}\right\}: 125.8 \mathrm{MHz},{ }^{19} \mathrm{~F}\left\{{ }^{1} \mathrm{H}\right\}: 470.6 \mathrm{MHz}\right)$ or on a Bruker Avance $400 \mathrm{NMR}$ spectrometer $\left({ }^{1} \mathrm{H}: 400.1 \mathrm{MHz},{ }^{11} \mathrm{~B}: 128.4 \mathrm{MHz},{ }^{13} \mathrm{C}\left\{{ }^{1} \mathrm{H}\right\}: 100.6 \mathrm{MHz},{ }^{14} \mathrm{~N}\right.$ : $\left.28.915 \mathrm{MHz},{ }^{19} \mathrm{~F}\left\{{ }^{1} \mathrm{H}\right\}: 376.5 \mathrm{MHz}\right)$ at $298 \mathrm{~K}$ unless otherwise stated. Chemical shifts $(\delta)$ are given in ppm and internally referenced to the carbon nuclei $\left({ }^{13} \mathrm{C}\left\{{ }^{1} \mathrm{H}\right\}\right)$ or residual protons $\left({ }^{1} \mathrm{H}\right)$ of the solvent. ${ }^{11} \mathrm{~B}$ NMR spectra were referenced to $\left[\mathrm{BF}_{3} \cdot \mathrm{OEt}_{2}\right],{ }^{19} \mathrm{~F}$ to $\mathrm{CFCl}_{3}$ and ${ }^{14} \mathrm{~N}$ to nitromethane as external standards. UV/Vis spectra were acquired on a JASCO-V660 UV/Vis spectrometer under inert conditions inside a glovebox. Solid-state IR spectra were acquired on a Bruker Alpha spectrometer using a setup with a Bruker diamond crystal single reflection ATR system. High-resolution mass spectrometry data was obtained from a Thermo Scientific Exactive Plus spectrometer in ASAP or LIFDI mode. Elemental analysis was conducted on an Elementar vario MICRO cube elemental analyser. Solvents and $\mathrm{TMSN}_{3}$ were purchased from Sigma-Aldrich or Alfa Aesar. $\mathrm{PhN}_{3},{ }^{1}$ $\mathrm{B}_{2} \mathrm{Cl}_{2} \mathrm{Mes}_{2},{ }^{2-3} \mathrm{~B}_{2} \mathrm{Br}_{2} \mathrm{Dur}_{2},{ }^{4} \mathrm{~B}_{2} \mathrm{Br}_{2} \mathrm{An}_{2}{ }^{5}$ and $\mathrm{F}_{2} \mathrm{~B}-\mathrm{BAn}_{2}{ }^{6}$ were synthesized by literature procedures. 


\section{Synthetic Procedures}

Synthesis of 1a

$$
\text { Dur- }-N^{-N}-\mathrm{Ph}
$$

$\operatorname{Dur}_{2} \mathbf{B}_{2} \mathbf{B r}_{2}$ (84.5 mg, $0.19 \mathrm{mmol}$ ) was dissolved in toluene (2.0 mL) and an excess of phenyl azide (35 $\mathrm{mg}, 0.29 \mathrm{mmol}$ ) was added. The solution turned from colorless to orange within a few minutes. After stirring for $4 \mathrm{~d}$ at room temperature, the resulted precipitate was isolated and washed twice with hexane $(2 \times 1.5 \mathrm{~mL})$ to yield compound $1 \mathrm{a}$ as an orange solid (55.3 mg, $0.10 \mathrm{mmol}, 51 \%)$. Crystals suitable for single-crystal X-ray diffraction were obtained by slow evaporation of a saturated benzene solution.

${ }^{1} \mathrm{H}$ NMR (400.1 MHz, $\left.\mathrm{C}_{6} \mathrm{D}_{6}\right): \delta=8.22-8.19\left(\mathrm{~m}, 2 \mathrm{H}, \mathrm{C}_{\text {phenyl- }}-H\right), 7.00-6.94\left(\mathrm{~m}, 3 \mathrm{H}, \mathrm{C}_{\text {phenyl- }}-H\right), 6.75$ (s, $1 \mathrm{H}, \mathrm{C}_{\text {Duryl- }} H$ ), $6.68\left(\mathrm{~s}, 1 \mathrm{H}, \mathrm{C}_{\text {Duryl }}-H\right), 2.48\left(\mathrm{~s}, 6 \mathrm{H}, \mathrm{CH}_{3}\right), 1.95$ (s, $\left.6 \mathrm{H}, \mathrm{CH}_{3}\right), 1.93\left(\mathrm{~s}, 6 \mathrm{H}, \mathrm{CH}_{3}\right), 1.81$ (s, $\left.6 \mathrm{H}, \mathrm{CH}_{3}\right) \mathrm{ppm}$.

${ }^{11} \mathrm{~B}$ NMR (128.4 MHz, $\left.\mathrm{C}_{6} \mathrm{D}_{6}\right): \delta=61.7$ (br s, $B$-Dur), $-2.2\left(\mathrm{~s}, B \mathrm{Br}_{2}\right) \mathrm{ppm}$.

${ }^{13} \mathrm{C}\left\{{ }^{1} \mathrm{H}\right\}$ NMR $\left(125.8 \mathrm{MHz}, \mathrm{C}_{6} \mathrm{D}_{6}\right): \delta=140.8\left(\mathrm{~s}, C_{\mathrm{q}}\right), 140.0\left(\mathrm{~s}, C_{\mathrm{q}}\right), 136.7\left(\mathrm{~s}, C_{\mathrm{q}}\right), 135.2\left(\mathrm{~s}, C_{\mathrm{q}}\right), 133.8$ $\left(\mathrm{s}, C_{\mathrm{q}}\right.$ ), 133.6 (s, duryl- $\mathrm{CH}$ ), 132.3 (s, duryl- $\mathrm{CH}$ ), 130.9 (s, $C_{\mathrm{q}}$ ), 130.8 (s, phenyl- $\mathrm{CH}$ ), 129.4 (phenyl$\mathrm{CH}$ ), 123.7 (phenyl- $\mathrm{CH}), 22.6\left(\mathrm{~s}, \mathrm{CH}_{3}\right), 19.9$ (s, $\left.\mathrm{CH}_{3}\right), 19.6$ (s, $\left.\mathrm{CH}_{3}\right), 15.9$ (s, $\left.\mathrm{CH}_{3}\right)$ ppm. Note: The resonance of one quaternary carbon atom could not be detected.

LIFDI-MS for [ $\left.\mathrm{C}_{26} \mathrm{H}_{31} \mathrm{~B}_{2} \mathrm{Br}_{2} \mathrm{~N}_{3}\right]$ : calcd: 567.1045; found: 567.1034 .

$\underline{\text { Synthesis of } \mathbf{1 b}}$

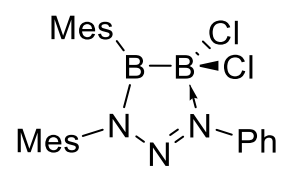

$\mathbf{M e s}_{2} \mathbf{B}_{2} \mathbf{C l}_{2}$ (78.5 mg, $0.24 \mathrm{mmol}$ ) and phenylazide (30.5 mg, $0.26 \mathrm{mmol}$ ) were dissolved in toluene $\left(1.5 \mathrm{~mL}\right.$ each) separately and cooled to $-50{ }^{\circ} \mathrm{C}$. After dropwise addition of phenyl azide to the diborane the reaction mixture was stirred at $-50{ }^{\circ} \mathrm{C}$ for $2 \mathrm{~h}$, at which point a color change to orange was observed. Afterwards the mixture was allowed to warm to ambient temperature and stirred for another $15 \mathrm{~h}$. After removal of all volatiles in vacuo the residue was dissolved in hexane and stored at $-30{ }^{\circ} \mathrm{C}$ overnight. The resulting crystals were isolated and dried in vacuo to yield compound $\mathbf{1 b}$ as orange crystals $(58.9 \mathrm{mg}, 0.13 \mathrm{mmol}, 55 \%)$, which were directly used for X-ray diffraction experiments. The compound was found to be very moisture sensitive, and signs of replacement of one 
chloride by one $\mathrm{OH}^{-}$group were found by IR spectroscopy and mass spectrometry in samples that were not freshly prepared.

${ }^{1} \mathrm{H}$ NMR (500.1 MHz, $\left.\mathrm{C}_{6} \mathrm{D}_{6}\right): \delta=8.14-8.11\left(\mathrm{~m}, 2 \mathrm{H}, \mathrm{C}_{\text {phenyl }}-H\right), 6.98-6.91\left(\mathrm{~m}, 3 \mathrm{H}, \mathrm{C}_{\text {phenyl }}-H\right), 6.59$ (s, $\left.2 \mathrm{H}, \mathrm{C}_{\text {Mesityl }}-H\right), 6.51\left(\mathrm{~s}, 2 \mathrm{H}, \mathrm{C}_{\text {Mesityl- }}-H\right), 2.48\left(\mathrm{~s}, 6 \mathrm{H}, \mathrm{CH}_{3}\right), 2.02\left(\mathrm{~s}, 3 \mathrm{H}, \mathrm{CH}_{3}\right), 2.00\left(\mathrm{~s}, 6 \mathrm{H}, \mathrm{CH}_{3}\right), 1.92$ (s, $\left.3 \mathrm{H}, \mathrm{CH}_{3}\right) \mathrm{ppm}$.

${ }^{11} \mathrm{~B}$ NMR $\left(160.5 \mathrm{MHz}, \mathrm{C}_{6} \mathrm{D}_{6}\right): \delta=63.4$ (br s, $\left.B-\mathrm{Mes}\right), 5.9\left(\mathrm{~s}, B \mathrm{Cl}_{2}\right) \mathrm{ppm}$.

${ }^{13} \mathrm{C}\left\{{ }^{1} \mathrm{H}\right\} \operatorname{NMR}\left(125.8 \mathrm{MHz}, \mathrm{C}_{6} \mathrm{D}_{6}\right): \delta=140.8\left(\mathrm{~s}, C_{\mathrm{q}}\right), 140.2\left(\mathrm{~s}, C_{\mathrm{q}}\right), 139.3\left(\mathrm{~s}, C_{\mathrm{q}}\right), 138.5\left(\mathrm{~s}, C_{\mathrm{q}}\right), 137.8$ $\left(\mathrm{s}, C_{\mathrm{q}}\right), 134.4\left(\mathrm{~s}, C_{\mathrm{q}}\right), 132.2$ (br s, $C_{\mathrm{q}}$ ), 131.0 (s, phenyl- $C \mathrm{H}$ ), 130.1 (s, mesityl- $C \mathrm{H}$ ), 129.5 (s, phenyl$\mathrm{CH}), 128.3$ (mesityl- $\mathrm{CH}), 123.2$ (phenyl- $\mathrm{CH}), 23.5\left(\mathrm{~s}, \mathrm{CH}_{3}\right), 21.2\left(\mathrm{~s}, \mathrm{CH}_{3}\right), 20.8\left(\mathrm{~s}, \mathrm{CH}_{3}\right), 18.9\left(\mathrm{~s}, \mathrm{CH}_{3}\right)$ ppm.

LIFDI-MS for $\left[\mathrm{C}_{24} \mathrm{H}_{27} \mathrm{~B}_{2} \mathrm{Cl}_{2} \mathrm{~N}_{3}\right]: \mathrm{m} / \mathrm{z}$ (calculated) $=449.1763 ; \mathrm{m} / \mathrm{z}$ (found): 449.1765 .

Synthesis of 2<smiles></smiles>

$\mathbf{A} \mathbf{n}_{2} \mathbf{B}_{2} \mathbf{F}_{2}$ (73.0 mg, $\left.0.18 \mathrm{mmol}\right)$ was dissolved in benzene (10 mL) and an excess of phenyl azide (105 $\mathrm{mg}, 0.89 \mathrm{mmol}$ ) was added, which yielded a color change of the reaction mixture from red to orange. After stirring for $4 \mathrm{~h}$ at room temperature all volatiles were removed in vacuo and the resulting orange residue was washed with benzene ( $3 \times 2 \mathrm{~mL})$ and hexane $(3 \times 2 \mathrm{~mL})$ to yield compound 2 as a yellow solid (57.6 mg, $0.09 \mathrm{mmol}, 51 \%)$. Single crystals suitable for X-ray diffraction analysis were obtained by slow evaporation of a saturated benzene solution at room temperature.

${ }^{1} \mathrm{H}$ NMR (400.1 MHz, d8-THF): $\delta=8.75$ (s, $1 \mathrm{H}$, aryl-CH), 8.30 (s, $1 \mathrm{H}$, aryl-CH), 8.28-8.25 (m, $4 \mathrm{H}$, aryl-CH), 8.17-8.15 (m, $2 \mathrm{H}$, aryl-CH), 7.86-7.84 (m, $2 \mathrm{H}$, aryl-CH), 7.72-7.68 (m, $2 \mathrm{H}$, aryl-CH), 7.60-7.53 (m, $4 \mathrm{H}$, aryl-CH), 7.38-7.34 (m, 2 H, aryl-CH), 7.18-7.16 (m, 2 H, aryl-CH), 7.03-7.00 (m, 2 H, aryl-CH), 6.78-6.65 (m, 6 H, aryl-CH) ppm.

${ }^{11} \mathrm{~B}$ NMR (128.4 MHz, d8 $\left.-\mathrm{THF}\right): \delta=31.5$ (br s, $\left.B-\mathrm{An}\right), 1.0\left(\mathrm{~s}, B \mathrm{~F}_{2}\right) \mathrm{ppm}$.

${ }^{13} \mathrm{C}\left\{{ }^{1} \mathrm{H}\right\}$ NMR (125.8 MHz, d 8 -THF): $\delta=143.9\left(\mathrm{~s}, C_{\mathrm{q}}\right), 143.9\left(\mathrm{~s}, C_{\mathrm{q}}\right), 135.2\left(\mathrm{~s}, C_{\mathrm{q}}\right), 134.9\left(\mathrm{~s}, C_{\mathrm{q}}\right)$, $132.6\left(\mathrm{~s}, C_{\mathrm{q}}\right), 131.8\left(\mathrm{~s}, C_{\mathrm{q}}\right), 129.8(\mathrm{~s}, 3 \mathrm{C}$, aryl- $\mathrm{CH}), 129.2(\mathrm{~s}, 2 \mathrm{C}$, aryl- $\mathrm{CH}), 129.2$ (s, $1 \mathrm{C}$, aryl- $\mathrm{CH}$, detected by DEPT135), 128.9 (s, $2 \mathrm{C}$, aryl- $\mathrm{CH}$ ), 128.7 (s, $2 \mathrm{C}$, aryl- $\mathrm{CH}$ ), 128.6 (s, $1 \mathrm{C}$, aryl- $\mathrm{CH}$ ), 128.3 (s, 2 C, aryl- $C H$ ), 128.1 (s, 2 C, aryl- $C H$ ), 126.9 (s, 2 C, aryl- $C H$ ), 126.7 (s, 2 C, aryl- $C H$ ), 126.5 (s, 2 
$\mathrm{C}$, aryl- $\mathrm{CH}), 125.7$ (s, $2 \mathrm{C}$, aryl- $C \mathrm{H}), 125.5$ (s, $1 \mathrm{C}$, aryl- $\mathrm{CH}), 125.5$ (s, $2 \mathrm{C}$, aryl-CH), 124.3 (m, $2 \mathrm{C}$, aryl-CH) ppm. Note: Some quaternary carbon resonances could not be detected due to broadness.

${ }^{19} \mathrm{~F}\left\{{ }^{1} \mathrm{H}\right\}$ NMR (376.5 MHz, $\mathrm{d}_{8}$-THF): $\delta=-136.96(\mathrm{~s}),-137.04$ (s) ppm.

LIFDI-MS [ $\left.\mathrm{C}_{40} \mathrm{H}_{28} \mathrm{~B}_{2} \mathrm{~N}_{4} \mathrm{~F}_{2}\right]: \mathrm{m} / \mathrm{z}$ (calculated) $=624.2463 ; \mathrm{m} / \mathrm{z}$ (found): 624.2469 .

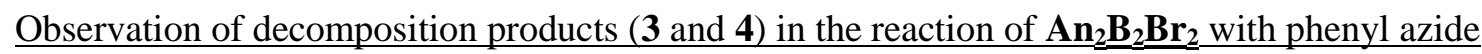

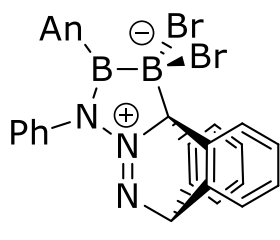

3

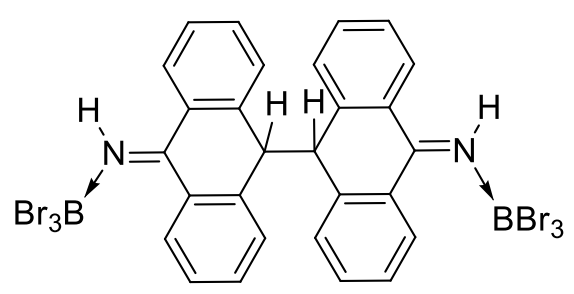

4

$\mathbf{A n}_{2} \mathbf{B}_{2} \mathbf{B} \mathbf{r}_{2}$ was suspended in $\mathrm{C}_{6} \mathrm{D}_{6}$ and an excess of phenyl azide was added without stirring. Upon addition the color of the solution as well as of the undissolved solid turned from orange to red and evolution of dinitrogen was observed by ${ }^{14} \mathrm{~N}$ NMR spectroscopy. $\mathrm{A}{ }^{11} \mathrm{~B}$ NMR spectrum of the reaction mixture showed complete consumption of the starting material and displayed multiple resonances at $\delta$ $=27.2,23.2,21.2$ (small), $-11.7,-11.9,-12.3$ and $-13.1 \mathrm{ppm}$. The ${ }^{1} \mathrm{H}$ NMR spectrum proved too complex to interpret. Upon standing for two days, the reaction mixture displayed new weak resonances by ${ }^{11} \mathrm{~B}$ NMR spectroscopy at $\delta=52.9$ and $40.0 \mathrm{ppm}$ along with the previously observed signals at $\delta=27.2,23.2$ and 21.3 (more intense now) and a new weak resonance at $\delta=-1.0 \mathrm{ppm}$ (all signals at ca. -12 ppm had disappeared).

In a subsequent experiment $\mathrm{B}_{2} \mathrm{Br}_{2} \mathrm{An}_{2}(23.0 \mathrm{mg} ; 0.04 \mathrm{mmol})$ was suspended in benzene $(0.9 \mathrm{~mL})$ and $\mathrm{PhN}_{3}$ (20 mg; $0.17 \mathrm{mmol}$ ) were added. Upon addition, crystals suitable for single-crystal X-ray diffraction precipitated as red needles which were found to be compound $\mathbf{3}$ (see X-ray Crystallographic Details below).

When $\mathrm{B}_{2} \mathrm{Br}_{2} \mathrm{An}_{2}$ was treated with $\mathrm{PhN}_{3}$ in toluene at $-50{ }^{\circ} \mathrm{C}$ new ${ }^{11} \mathrm{~B}$ NMR resonances were observed at $\delta=64.1,39.9,27.3$ and $-1.76 \mathrm{ppm}$. After drying in vacuo the ${ }^{11} \mathrm{~B}$ NMR spectrum at room temperature showed resonances at $\delta=40.4,29.1$ and sharp signals at $-6.71,-9.70$ and $-10.4 \mathrm{ppm}$. The ${ }^{1} \mathrm{H}$ NMR spectrum however, predominantly showed resonances corresponding to free anthracene (i.e. decomposition).

When $\mathrm{B}_{2} \mathrm{Br}_{2} \mathrm{An}_{2}(20.0 \mathrm{mg} ; 0.04 \mathrm{mmol})$ was treated with $\mathrm{PhN}_{3}(5.5 \mathrm{mg} ; 0.05 \mathrm{mmol})$ in benzene $(0.8$ $\mathrm{mL}$ ) and the reaction mixture was left standing in a glovebox for 11 days, the removed supernatant solution showed ${ }^{11} \mathrm{~B}$ resonances at $\delta=63.4,39.8,27.7,22.7,-12.2,-13.0 \mathrm{ppm}$. Evaporation of this 
solution in a glovebox yielded orange block crystals, which were found to be 4 ( $\mathrm{BBr}_{3}$ adduct) by $\mathrm{X}$-ray diffraction (see X-ray Crystallographic Details below).

The high resolution mass spectrometry showed peaks corresponding to meaningful products and intermediates:

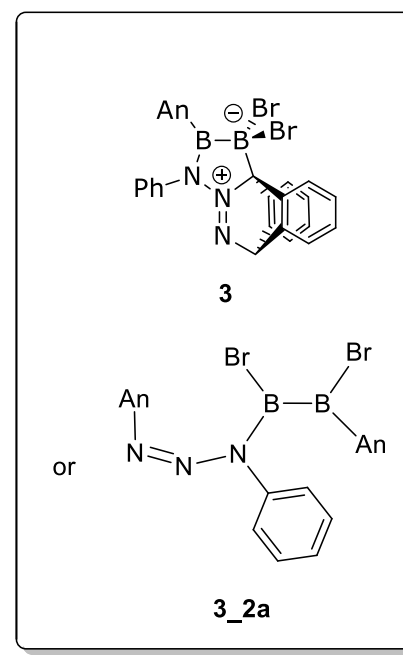

LIFDI-MS : $\mathrm{m} / \mathrm{z}$ (calculated) = 655.0419; $\mathrm{m} / \mathrm{z}$ (found):655.0414.

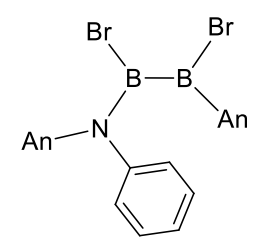

$$
\begin{gathered}
\text { LIFDI-MS }: \mathrm{m} / \mathrm{z} \text { (calculated) }=627.0363 \\
\mathrm{~m} / \mathrm{z} \text { (found) } 627.0349
\end{gathered}
$$

3_2c

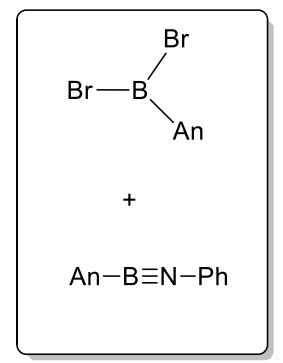

LIFDI-MS : $\mathrm{m} / \mathrm{z}$ (calculated) $=347.9144$; $\mathrm{m} / \mathrm{z}$ (found): 347.9137

3_2b

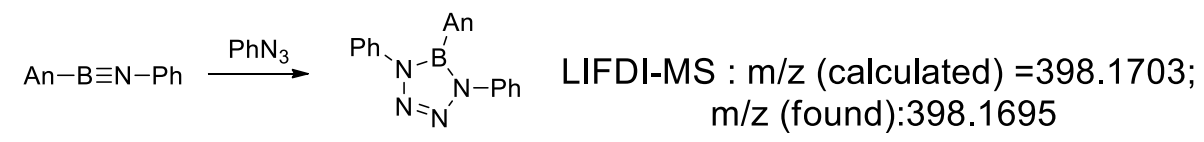

Figure S1: Mass spectrometry evidence for the presence of several proposed products and intermediates in the reaction of $\mathbf{A} \mathbf{n}_{2} \mathbf{B}_{2} \mathbf{B} \mathbf{r}_{2}$ with $\mathrm{PhN}_{3}$ (see Scheme S3).

\section{$\underline{\text { Isolation of } 6 \mathbf{a}(\mathbf{8})}$}


<smiles>C[SiH2]N1N=NN(B(C)[AsH3])B1C</smiles>

$6 a$

Not observed in the solid state

Observed by ${ }^{11} \mathrm{~B}$ NMR and MS

$\mathbf{M e s}_{2} \mathbf{B}_{2} \mathbf{C l}_{2}(48 \mathrm{mg}, 0.14 \mathrm{mmol})$ and trimethylsilyl azide $(50 \mathrm{mg}, 0.43 \mathrm{mmol})$ were dissolved in deuterated benzene and the reaction monitored by NMR spectroscopy. After $c a$. two weeks at room temperature, the mixture was evaporated to dryness, yielding a yellow oil from which a white solid formed. The mixture was washed with pentane, yielding the tetrazaborole- $\mathrm{B}\left(\mathrm{N}_{3}\right) \mathrm{Mes}(14 \mathrm{mg}, 0.032$ mmol, 23\%).

${ }^{1} \mathrm{H}$ NMR (400.1 MHz, $\left.\mathrm{C}_{6} \mathrm{D}_{6}\right): \delta=6.79(\mathrm{~s}, 2 \mathrm{H}, \mathrm{Mes}-H), 6.69$ (s, $\left.2 \mathrm{H}, \mathrm{Mes}-H\right), 2.21(\mathrm{~s}, 6 \mathrm{H}, \mathrm{Mes} o-$ $\mathrm{CH}_{3}$ ), 2.18 (s, $3 \mathrm{H}$, Mes $p$ - $\mathrm{CH}_{3}$ ), 2.15 (s, $6 \mathrm{H}$, Mes $o$-CH$H_{3}$ ), 2.12 (s, $3 \mathrm{H}$, Mes $p$ - $\mathrm{CH}_{3}$ ), 0.12 (s, $9 \mathrm{H}$, $\left.\mathrm{Si} M e_{3}\right) \mathrm{ppm}$.

${ }^{11} \mathrm{~B}$ NMR (128.4 MHz, $\left.\mathrm{C}_{6} \mathrm{D}_{6}\right): \delta=43.3\left(-B\left(\mathrm{~N}_{3}\right) \mathrm{Mes}\right), 32.9(\mathrm{~N}-B(\mathrm{Mes})-\mathrm{N}) \mathrm{ppm}$.

${ }^{13} \mathrm{C}\left\{{ }^{1} \mathrm{H}\right\}$ NMR (100.6 MHz, $\left.\mathrm{C}_{6} \mathrm{D}_{6}\right): \delta=140.18$ (Aryl- $C_{\mathrm{q}}$ ), 139.01 (Aryl- $C_{\mathrm{q}}$ ), 138.71 (Aryl- $C_{\mathrm{q}}$ ), 138.60 (Aryl- $C_{\mathrm{q}}$ ), 127.89 (Aryl- $\mathrm{CH}$ ), 127.64 (Aryl- $\mathrm{CH}$ ), $23.08\left(\mathrm{Mes}-\mathrm{CH}_{3}\right), 22.08\left(\mathrm{Mes}-\mathrm{CH}_{3}\right), 21.41$ (Mes$\left.\mathrm{CH}_{3}\right), 21.33\left(\right.$ Mes- $\left.\mathrm{CH}_{3}\right),-0.52\left(\mathrm{Si}\left(\mathrm{CH}_{3}\right)_{3} \mathrm{ppm}\right.$. The resonances of two expected B-C $\mathrm{C}_{\mathrm{q}}$ nuclei were not observed, presumably due to their broadness.

ASAP-MS $\left[\mathrm{C}_{21} \mathrm{H}_{31} \mathrm{~B}_{2} \mathrm{~N}_{7} \mathrm{Si}(\mathbf{6 a})+\mathrm{H}^{+}\right]: \mathrm{m} / \mathrm{z}$ (calculated) $=432.2669 ; \mathrm{m} / \mathrm{z}$ (found): 432.2660 .

\section{Isolation of $\mathbf{6 b}$}<smiles>C[SiH]1N=NN(B(N)Br)B1Br</smiles>

6b

$\operatorname{Dur}_{2} \mathbf{B}_{2} \mathbf{B r}_{2}(196 \mathrm{mg}, 0.44 \mathrm{mmol})$ and trimethylsilyl azide $(517 \mathrm{mg}, 4.50 \mathrm{mmol})$ were dissolved in dichloromethane. After $c a$. one week at room temperature, the mixture was evaporated to dryness, yielding a pale yellow glassy material. This was washed with pentane, yielding $\mathbf{6 b}$ as a white powder. More white material could be obtained by cooling the pentane washings at $-30{ }^{\circ} \mathrm{C}$, from which colorless crystals formed (combined yields $96 \mathrm{mg}, 48 \mathrm{~mol} \%$ based on $\operatorname{Dur}_{2} \mathbf{B}_{2} \mathbf{B r} \mathbf{r}_{2}$ ), which were used for X-ray diffraction experiments (see X-ray Crystallographic Details below).

${ }^{1} \mathrm{H}$ NMR (400.1 MHz, $\left.\mathrm{C}_{6} \mathrm{D}_{6}\right): \delta=6.82(\mathrm{~s}, 1 \mathrm{H}, \mathrm{Dur}-H), 6.78$ (s, $\left.1 \mathrm{H}, \mathrm{Dur}-H\right), 2.11$ (s, $\left.6 \mathrm{H}, \mathrm{Dur}-\mathrm{CH}_{3}\right)$, 2.06 (s, $6 \mathrm{H}$, Dur-CH ), 2.01 (s, $6 \mathrm{H}$, Dur- $\mathrm{CH}_{3}$ ), 1.96 (s, $6 \mathrm{H}, \mathrm{Mes}$ - $-\mathrm{CH}_{3}$ ), 0.13 (s, $9 \mathrm{H},-\mathrm{SiMe}$ ) ppm. 
${ }^{11} \mathrm{~B}$ NMR (128.4 MHz, $\left.\mathrm{C}_{6} \mathrm{D}_{6}\right): \delta=43.8\left(-B\left(\mathrm{~N}_{3}\right) \mathrm{Mes}\right), 33.5(\mathrm{~N}-B(\mathrm{Mes})-\mathrm{N}) \mathrm{ppm}$.

${ }^{13} \mathrm{C}\left\{{ }^{1} \mathrm{H}\right\}$ NMR (100.6 MHz, $\mathrm{C}_{6} \mathrm{D}_{6}$ ): 135.88 (aryl-C), 134.21 (aryl-C), 133.52 (aryl-C), 133.14 (aryl-C), 132.82 (aryl-C), 132.53 (aryl-C), 20.68 (Duryl- $\mathrm{CH}_{3}$ ), 19.68 (Duryl- $\mathrm{CH}_{3}$ ), 19.64 (Duryl- $\mathrm{CH}_{3}$ ), 19.39 (Duryl- $\left.\mathrm{CH}_{3}\right),-0.52\left(\mathrm{Si}\left(\mathrm{CH}_{3}\right)_{3}\right) \mathrm{ppm}$. The two expected B-C $\mathrm{C}_{\mathrm{q}}$ were not observed, presumably due to their broadness.

ASAP-MS $\left[\mathrm{C}_{23} \mathrm{H}_{35} \mathrm{~N}_{7} \mathrm{~B}_{2} \mathrm{Si}+\mathrm{H}^{+}\right]: \mathrm{m} / \mathrm{z}$ (calculated): 460.2982; m/z (found) 460.2976 .

\section{Detection of $\mathbf{5 a}$ and $\mathbf{8}$}

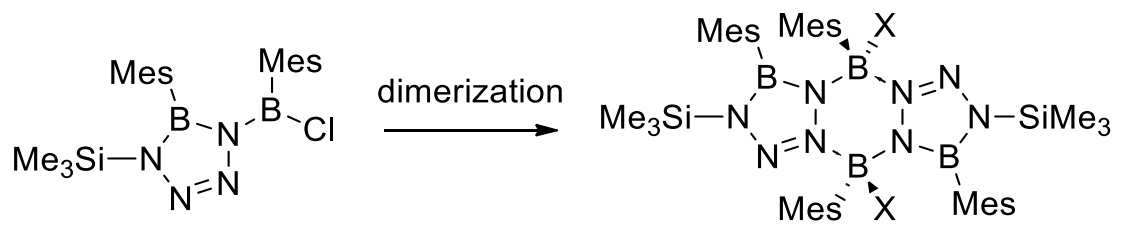

$5 \mathbf{a}$

Not observed in the solid state
Observed by X-ray diffraction with $\mathrm{X}=\mathrm{Cl} /\left(\mathrm{N}_{3}\right)$ Possibly small amounts observed by ${ }^{11} \mathrm{~B}$ NMR in solution

$\mathbf{M e s}_{2} \mathbf{B}_{2} \mathbf{C l}_{2}(22 \mathrm{mg}, 0.066 \mathrm{mmol})$ and trimethylsilyl azide $(18 \mathrm{mg}, 0.16 \mathrm{mmol})$ were dissolved in deuterated benzene and the reaction was monitored by NMR spectroscopy. The reaction appeared to be complete within a few days at room temperature. The volatile components were removed in vacuo, leaving behind a pale yellow oil from which a white solid formed. Combined extracts in pentane, toluene and dichloromethane were slowly evaporated in the inert atmosphere of a glovebox, yielding crystalline material that X-ray diffraction experiments showed to be the dimer of a mixed -B(Mes)Cl/ $\mathrm{B}(\mathrm{Mes})\left(\mathrm{N}_{3}\right)$-substituted tetrazaborole (see X-ray Crystallographic Details below).

In addition to the typical signals for 5a and 6a $\left({ }^{11} \mathrm{~B}\right.$ NMR: $50.7(-B(\mathrm{Cl}) \mathrm{Mes}, \mathbf{5 a}), 43.0\left(-B\left(\mathrm{~N}_{3}\right) \mathrm{Mes}, \mathbf{6 a}\right)$, $34.0(\mathrm{~N}-B(\mathrm{Mes})-\mathrm{N}$ ppm, 5a/6a)), an additional weak and broad signal at $6.1 \mathrm{ppm}$ was observed, which is consistent with the expected signal of the bridging $\operatorname{MesB}(\mathrm{X})$ moieties in $\mathbf{8}$.

\section{Isolation and detection of 7 by X-ray crystallography}

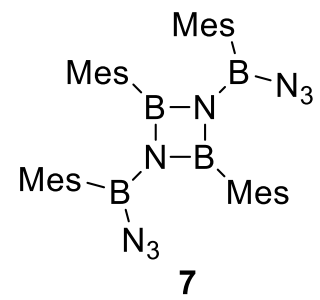


$\mathbf{M e s}_{2} \mathbf{B}_{2} \mathbf{B r}_{2}(23 \mathrm{mg}, 0.055 \mathrm{mmol})$ and trimethylsilyl azide $(70 \mathrm{mg}, 0.61 \mathrm{mmol})$ were dissolved in benzene. After $c a .24 \mathrm{~h}$ at room temperature, a mixture with the following spectroscopic properties was obtained:

${ }^{11} \mathrm{~B}$ NMR (128.4 MHz, $\left.\mathrm{C}_{6} \mathrm{D}_{6}\right): \delta=43.0\left(-B\left(\mathrm{~N}_{3}\right) \mathrm{Mes}\right), 36.5(\mathrm{~N}-B(\mathrm{Mes})-\mathrm{N}), 37.6$ (sharp, possibly a signal belonging to a diazadiboretidine derivative), 5.4 (small, consistent with the expected chemical shift of the tetrazaborole dimer $\mathbf{8}$ ).

These spectroscopic features (as well as ${ }^{1} \mathrm{H}$ NMR signals) are consistent with a mixture containing mostly $\mathrm{B}\left(\mathrm{N}_{3}\right)$ Mes-tetrazaborole $(\mathbf{6 a})$ and a second species. The mixture was evaporated to dryness, yielding a pale yellow glassy material, which was recrystallized from pentane. The crystallized solid was found to be significantly richer in the secondary product and a crystal structure obtained by X-ray diffraction data proved the presence of $(\mathrm{B}(\mathrm{X}) \mathrm{Mes})_{2}$-diazadiboretidine (7) in the mixture (see X-ray Crystallographic Details below). After washing the recrystallized material with pentane, the secondary product, which we assign to the diazadiboretidine (7), was isolated in small amounts:

${ }^{1} \mathrm{H}$ NMR (400.1 MHz, $\left.\mathrm{C}_{6} \mathrm{D}_{6}\right): \delta=6.59(\mathrm{~s}, 2 \mathrm{H}$, Mes- $H$ ), $6.45(\mathrm{~s}, 2 \mathrm{H}, \mathrm{Mes}-H), 2.41(\mathrm{~s}, 6 \mathrm{H}, \mathrm{Mes} o-$ $\mathrm{CH}_{3}$ ), 2.18 (s, $6 \mathrm{H}$, Mes $o-\mathrm{CH}_{3}$ ), 2.03 (s, $6 \mathrm{H}$, Mes $p$ - $\mathrm{CH}_{3}$ ), 1.95 (s, $6 \mathrm{H}$, Mes $p$-CH $\mathrm{CH}_{3}$ ) ppm.

${ }^{11} \mathrm{~B}$ NMR (128.4 MHz, $\mathrm{C}_{6} \mathrm{D}_{6}$ ): $\delta=$ broad and very weak features that would most likely be obscured in mixtures: 50.9, 40.8 (see spectrum below) ppm.

${ }^{13} \mathrm{C}\left\{{ }^{1} \mathrm{H}\right\}$ NMR (100.6 MHz, $\mathrm{C}_{6} \mathrm{D}_{6}$ ): $\delta=138.7$ (s, Mes- $C_{\mathrm{q}}$ ), 138.63 (s, Mes- $C_{\mathrm{q}}$ ), 138.16 (s, Mes- $C_{\mathrm{q}}$ ), 138.01 (s, Mes- $C_{\mathrm{q}}$ ), 127.31 (s, Mes- $C H$ ), 127.14 (s, Mes- $C H$ ), 22.71 (s, Mes $o-C_{3}$ ), 22.07 (s, Mes o$\mathrm{CH}_{3}$ ), 21.31 (s, Mes $\left.p-\mathrm{CH}_{3}\right), 21.15$ (s, Mes $\left.p-\mathrm{CH}_{3}\right) \mathrm{ppm}$. The two expected resonances for $\mathrm{B}-\mathrm{C} \mathrm{C}_{\mathrm{q}}$ nuclei were not observed, presumably due to their broadness.

ASAP-MS $\left[\mathrm{C}_{36} \mathrm{H}_{44} \mathrm{~B}_{4} \mathrm{~N}_{8}(7)+\mathrm{H}^{+}\right]: \mathrm{m} / \mathrm{z}$ (calculated): $632.4170 ; \mathrm{m} / \mathrm{z}$ (found) 632.4158 .

\section{$\underline{\text { Observation of } \mathbf{5 b}}$}<smiles>C[AsH]N1N=NN(B(C)C)B1C</smiles>

$5 \mathbf{b}$

detected by NMR and MS

$\mathbf{M e s}_{2} \mathbf{B}_{2} \mathbf{B r}_{2}(31 \mathrm{mg}, 0.074 \mathrm{mmol})$ and trimethylsilyl azide $(17 \mathrm{mg}, 0.15 \mathrm{mmol})$ were dissolved in benzene. After $c a .24 \mathrm{~h}$ at room temperature, the mixture was evaporated to dryness, yielding a pale yellow glassy material, in which the presence of a mixture of $\mathrm{B}\left(\mathrm{N}_{3}\right)$ Mes-tetrazaborole (6a) and 
$\mathrm{B}(\mathrm{Br})$ Mes-tetrazaborole (5b) was detected by ${ }^{11} \mathrm{~B}$ NMR and mass spectrometry. The ${ }^{1} \mathrm{H}$ NMR spectrum suggested the presence of a complex mixture, which was not further characterized.

${ }^{11} \mathrm{~B}$ NMR (128.4 MHz, $\left.\mathrm{C}_{6} \mathrm{D}_{6}\right): \delta=52.7$ (-B(Br)Mes, 5b), 34.8 (N-B(Mes)-N, 5b), 5.7 (traces, consistent with the expected chemical shift of the tetrazaborole dimer bridging $\mathrm{B}(\mathrm{X})$ Mes group in $\mathbf{8}$ ) ppm.

ASAP-MS $\left[\mathrm{C}_{21} \mathrm{H}_{31} \mathrm{~B}_{2} \mathrm{BrN}_{4} \mathrm{Si}(\mathbf{5 b})+\mathrm{H}^{+}\right]: \mathrm{m} / \mathrm{z}$ (calculated) $=471.1740 ; \mathrm{m} / \mathrm{z}$ (found): 471.1731.

\section{Detection of $\mathbf{5 c}$}

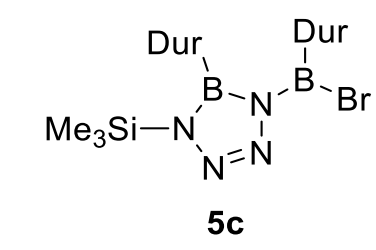

Detected by ${ }^{11} \mathrm{~B}$ NMR and MS

$\operatorname{Dur}_{2} \mathbf{B}_{2} \mathbf{B r}_{2}(62 \mathrm{mg}, 0.14 \mathrm{mmol})$ and trimethylsilyl azide $(32 \mathrm{mg}, 0.28 \mathrm{mmol})$ were dissolved in benzene. After $c a .24 \mathrm{~h}$ at room temperature, the mixture was evaporated to dryness, yielding a pale yellow glassy material, which was found to contain $\mathbf{5 c}$ by mass spectrometry and ${ }^{11} \mathrm{~B}$ NMR spectroscopy. The ${ }^{1} \mathrm{H}$ NMR spectrum of the resulting material was relatively complex, suggesting a complex mixture with one major component.

${ }^{11} \mathrm{~B}$ NMR (128.4 MHz, $\left.\mathrm{C}_{6} \mathrm{D}_{6}\right): \delta=51.5$ (-B(Br)Dur), 34.8 (N-B(Dur)-N), 5 (traces, consistent with the expected chemical shift of the tetrazaborole dimer bridging $\mathrm{B}(\mathrm{X})$ Dur group in 8-like dimers) ppm.

ASAP-MS $\left[\mathrm{C}_{23} \mathrm{H}_{35} \mathrm{~B}_{2} \mathrm{BrN}_{4} \mathrm{Si}(\mathbf{5 c})+\mathrm{H}^{+}\right]: \mathrm{m} / \mathrm{z}$ (calculated) $=499.2053 ; \mathrm{m} / \mathrm{z}$ (found): 499.2043. 


\section{$\underline{\text { NMR Spectra }}$}

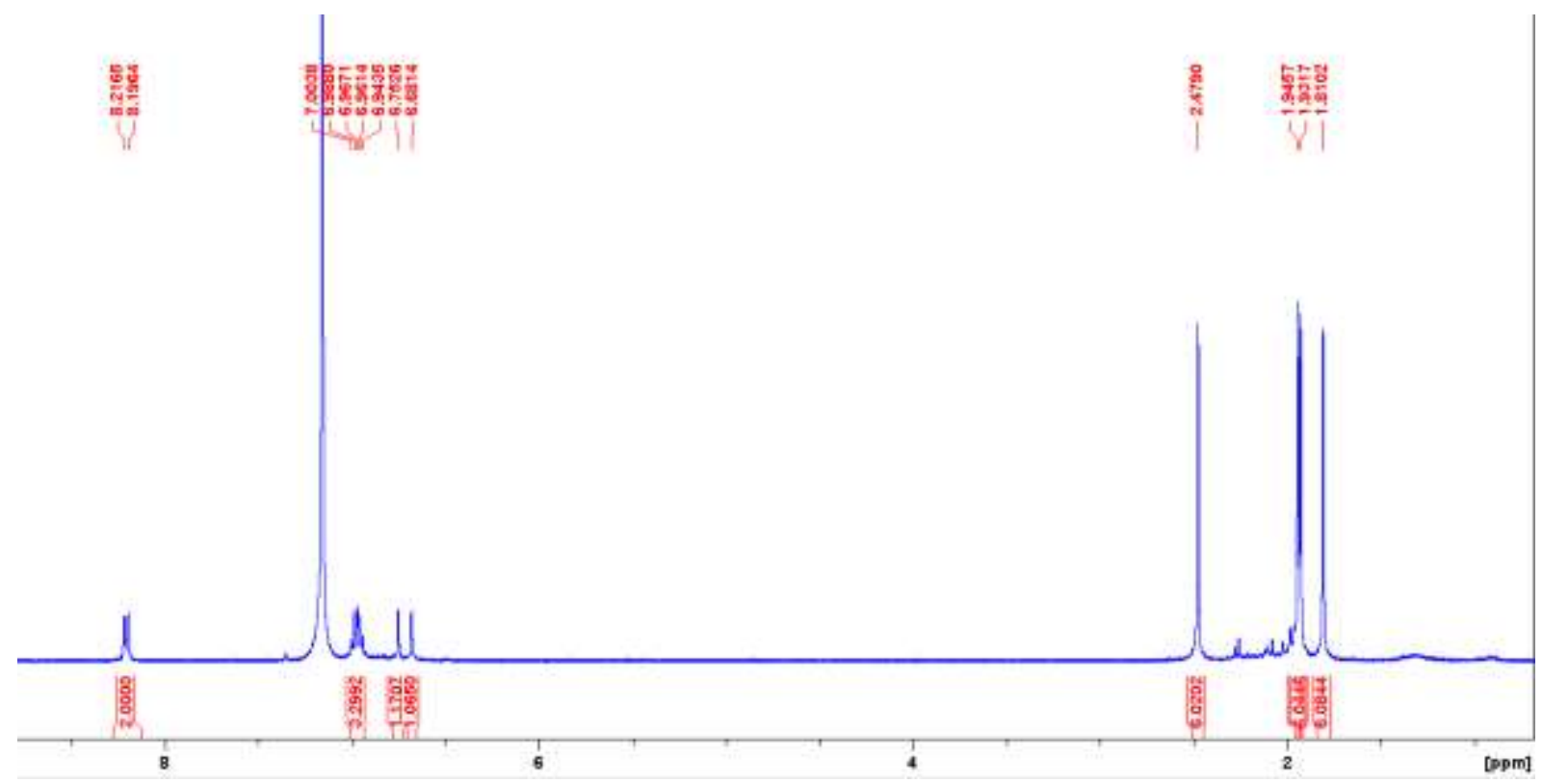

Figure S2: ${ }^{1} \mathrm{H}$ NMR spectrum of $\mathbf{1 a}$ in $\mathrm{C}_{6} \mathrm{D}_{6}$.

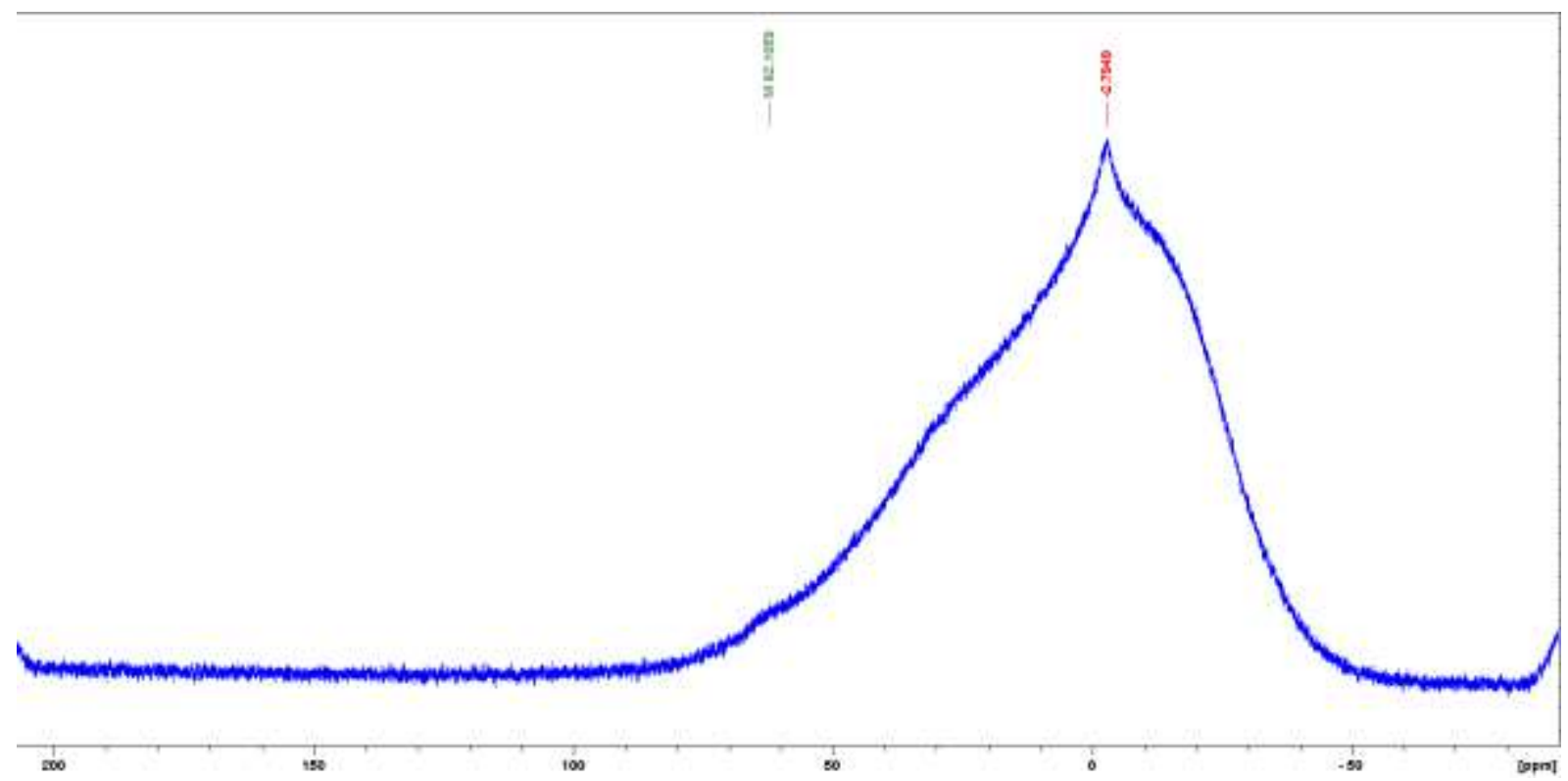

Figure S3: ${ }^{11} \mathrm{~B}$ NMR spectrum of $\mathbf{1 a}$ in $\mathrm{C}_{6} \mathrm{D}_{6}$. Note: Compound $\mathbf{1 a}$ is poorly soluble in $\mathrm{C}_{6} \mathrm{D}_{6}$ resulting in very weak resonances in the ${ }^{11} \mathrm{~B}$ NMR spectrum in conjunction with broadness of the resonance of the tricoordinate boron atom. However, deuterated non-aromatic solvents lead to decomposition. 


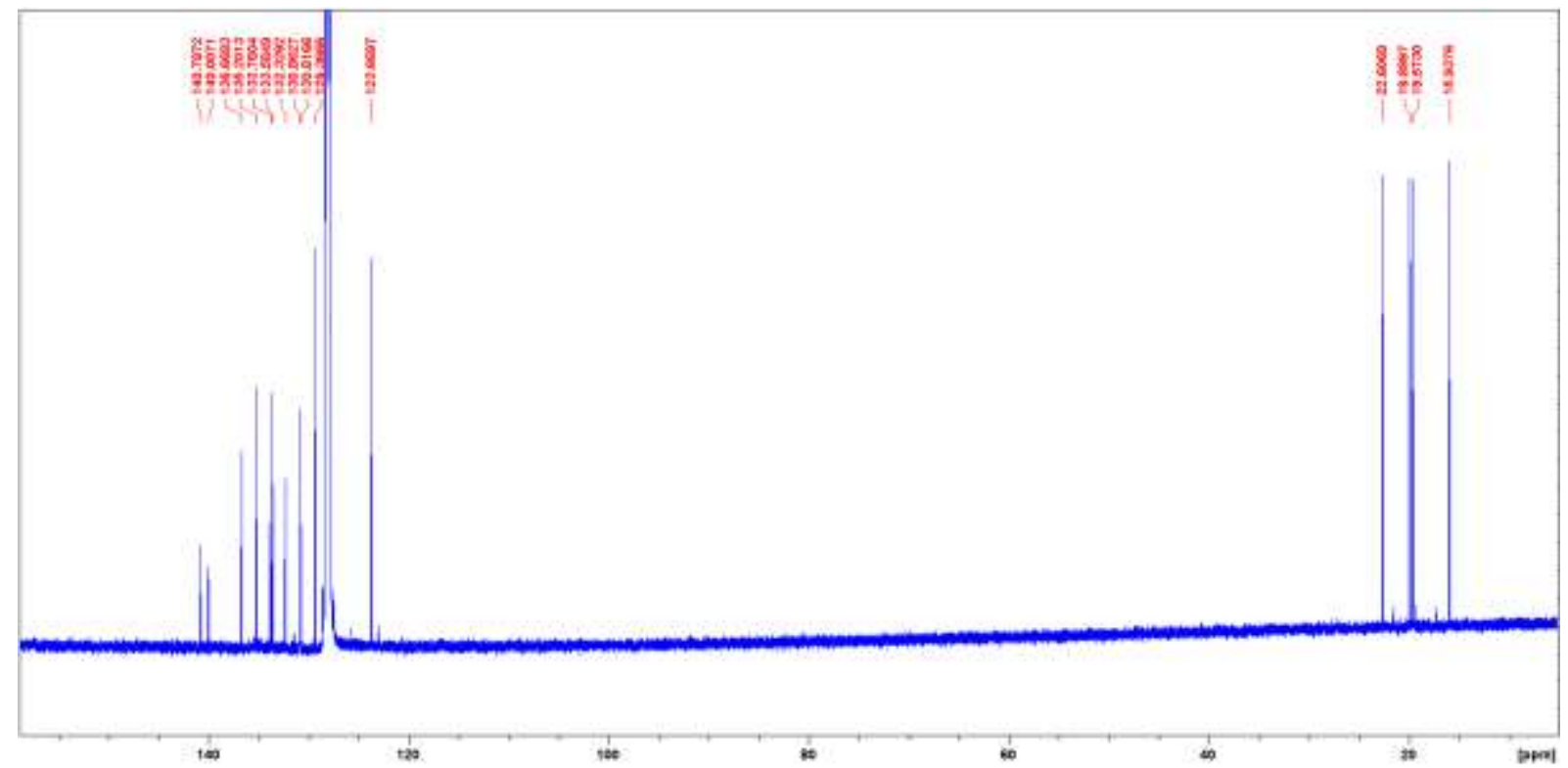

Figure $\mathrm{S} 4:{ }^{13} \mathrm{C}\left\{{ }^{1} \mathrm{H}\right\}$ NMR spectrum of $\mathbf{1 a}$ in $\mathrm{C}_{6} \mathrm{D}_{6}$.

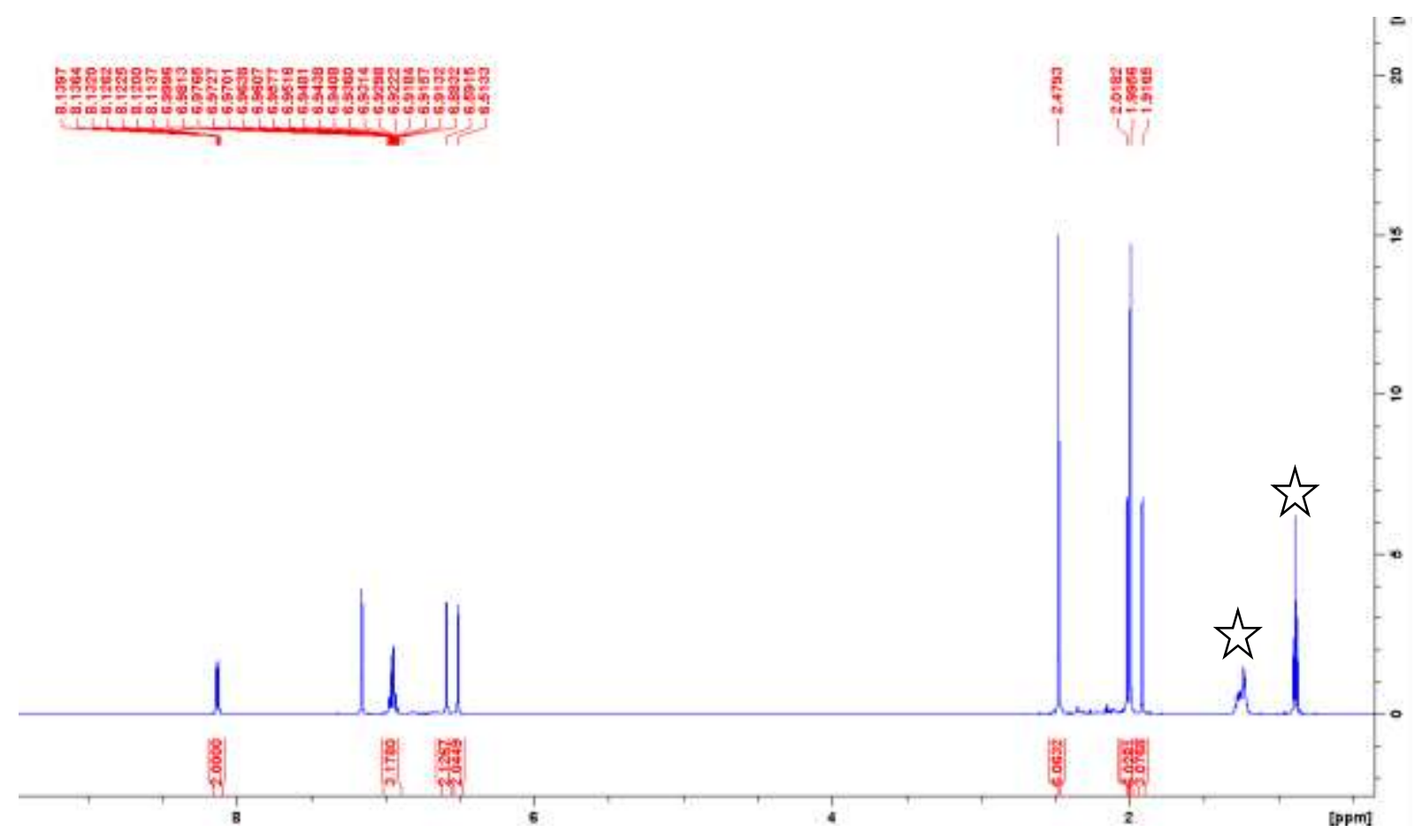

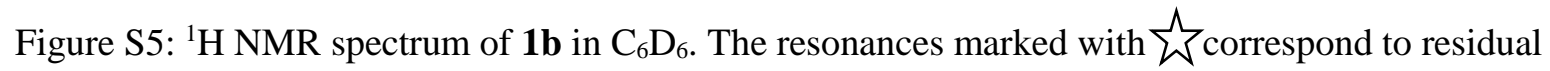
hexane. 


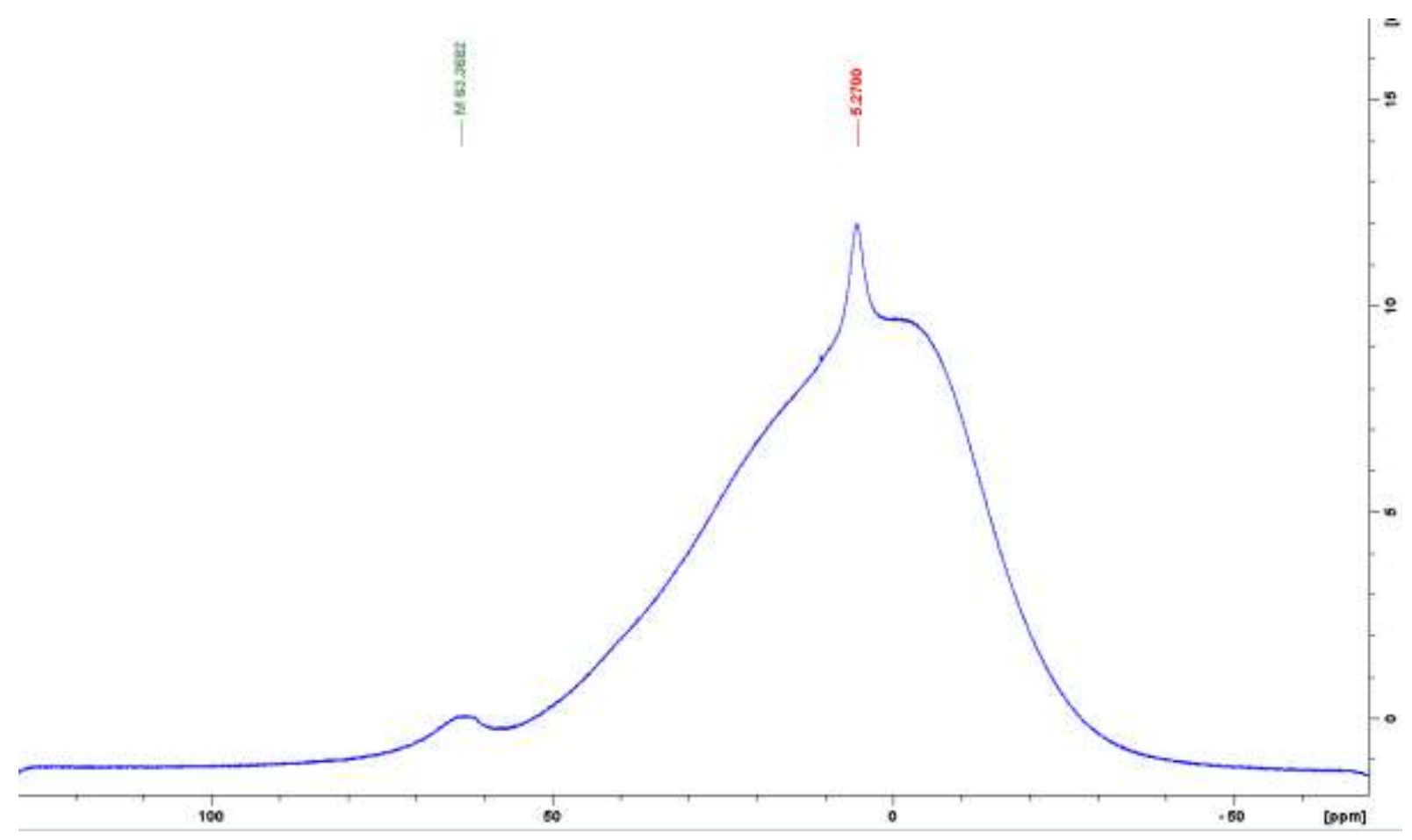

Figure S6: ${ }^{11} \mathrm{~B}$ NMR spectrum of $\mathbf{1 b}$ in $\mathrm{C}_{6} \mathrm{D}_{6}$.

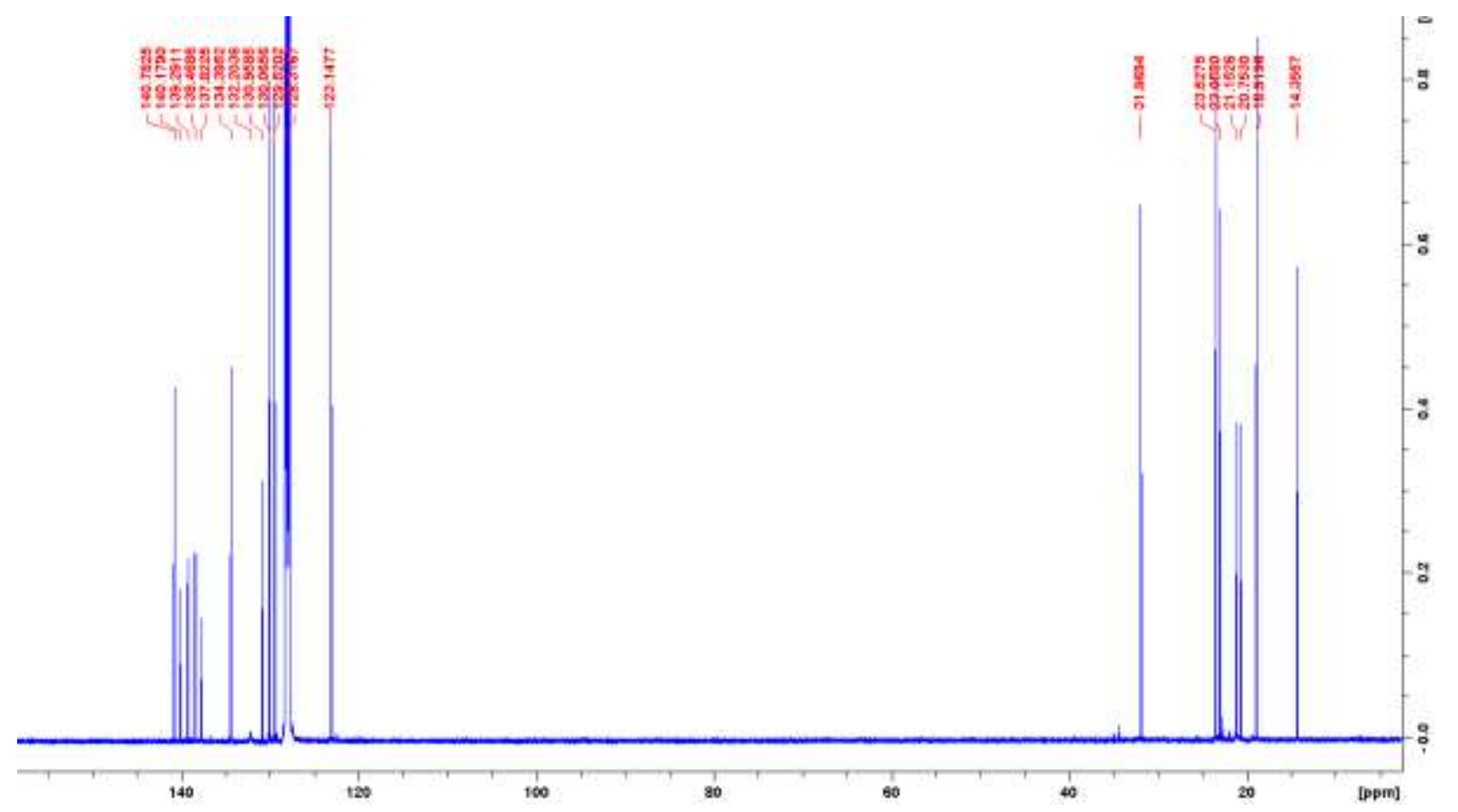

Figure S7: ${ }^{13} \mathrm{C}\left\{{ }^{1} \mathrm{H}\right\}$ NMR spectrum of $\mathbf{1 b}$ in $\mathrm{C}_{6} \mathrm{D}_{6}$. The signals at 32.0, 23.1 and $14.4 \mathrm{ppm}$ correspond to residual hexane. 


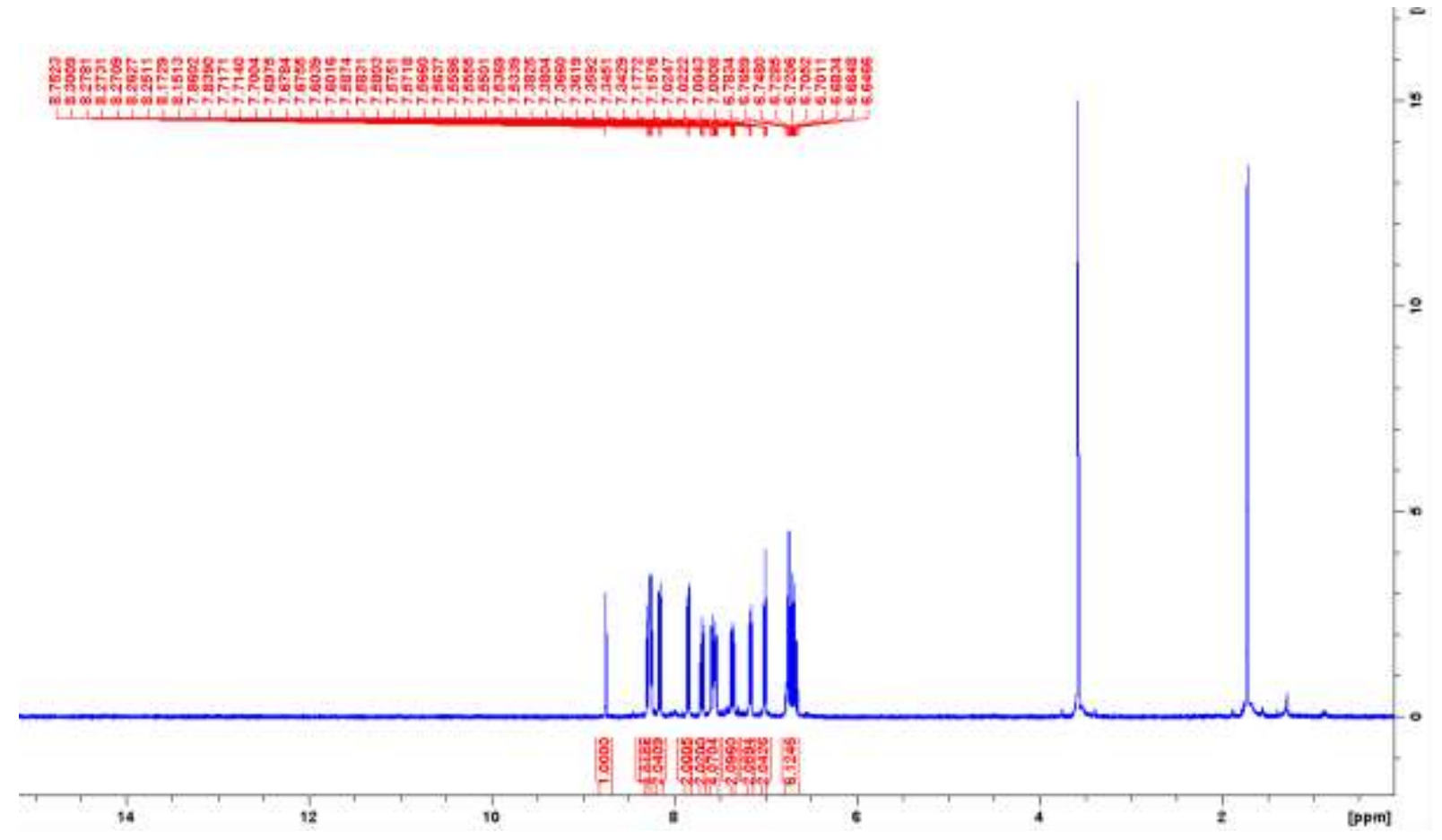

Figure S8: ${ }^{1} \mathrm{H}$ NMR spectrum of 2 in $\mathrm{d}_{8}$-THF.

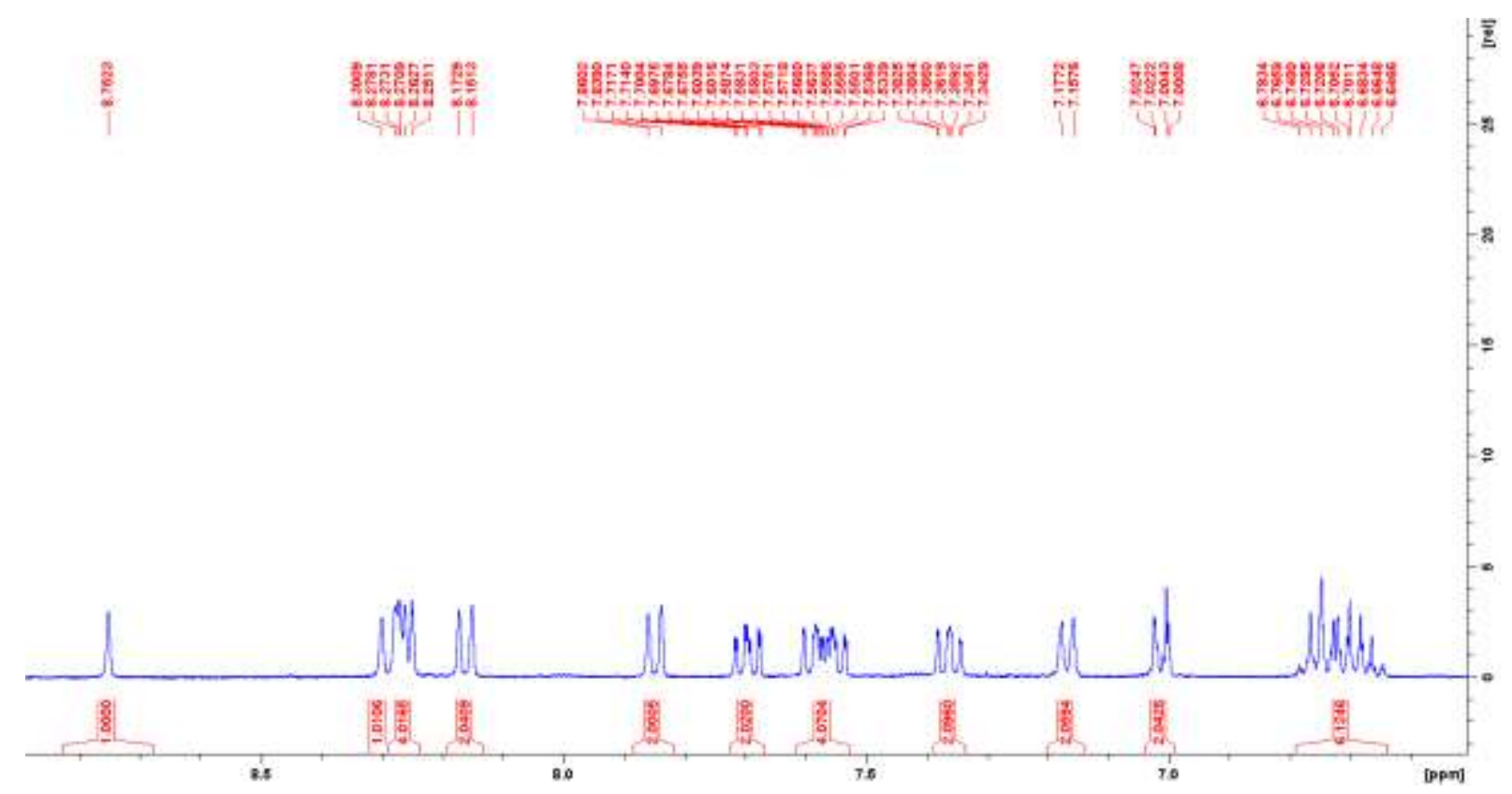

Figure S9: Downfield excerpt of the ${ }^{1} \mathrm{H}$ NMR spectrum of 2 in $\mathrm{d}_{8}$-THF. 


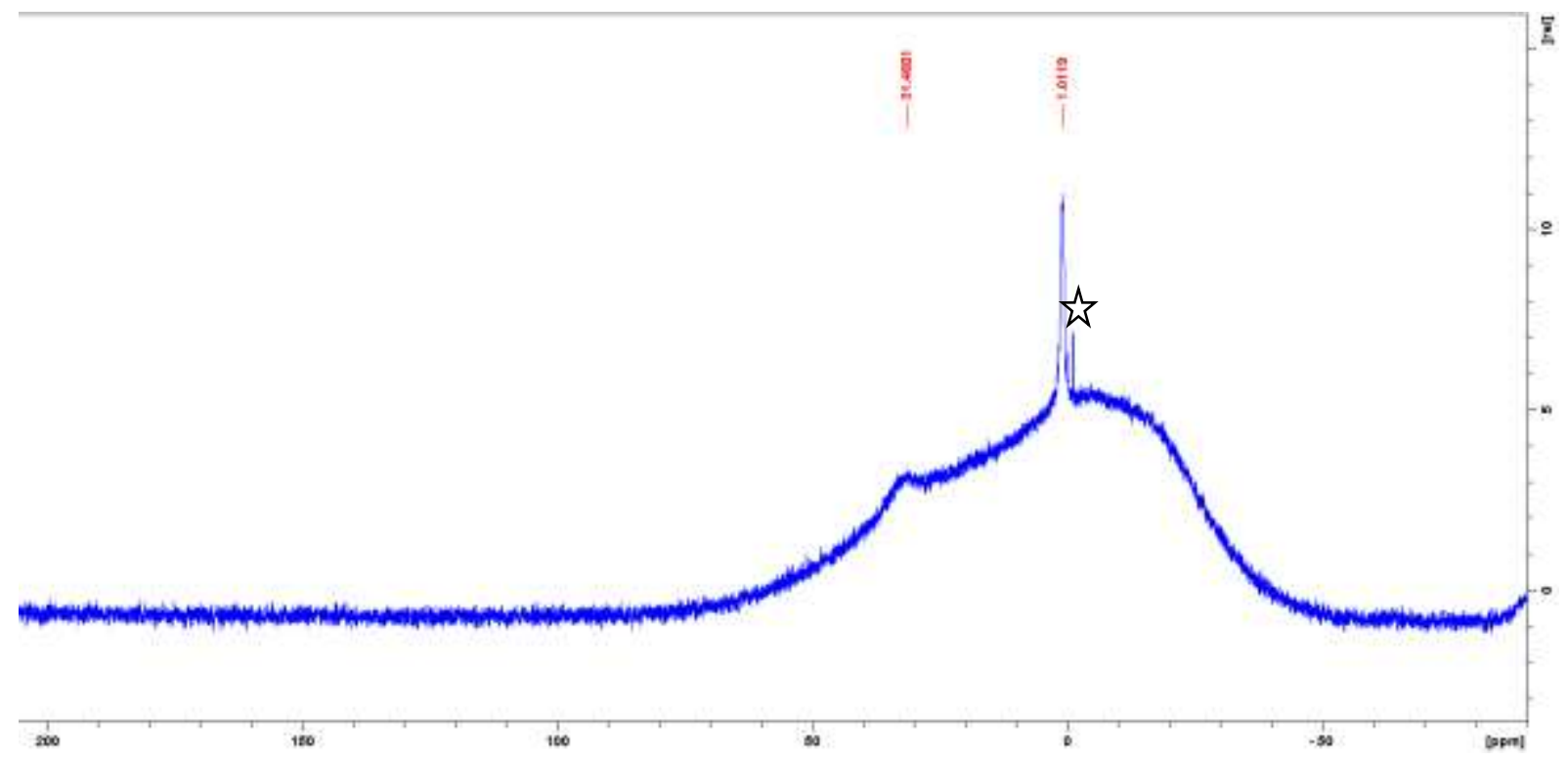

Figure S10: ${ }^{11} \mathrm{~B}$ NMR spectrum of 2 in $\mathrm{d}_{8}$-THF. The resonance marked with $2 \hat{\gamma}$ (at $-1.0 \mathrm{ppm}$ ) corresponds to an unknown impurity.

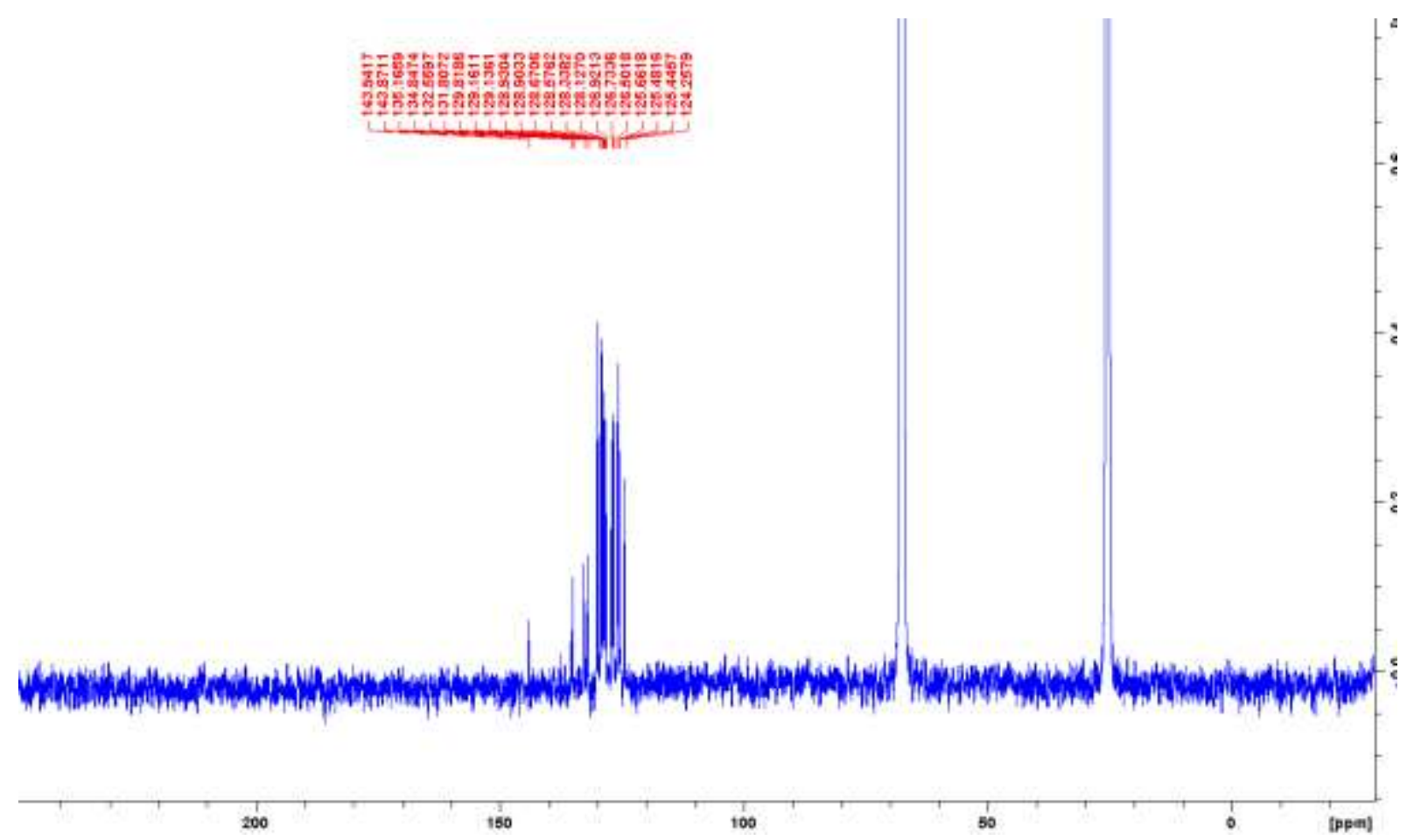

Figure S11: ${ }^{13} \mathrm{C}\left\{{ }^{1} \mathrm{H}\right\}$ NMR spectrum of 2 in $\mathrm{d}_{8}$-THF. 


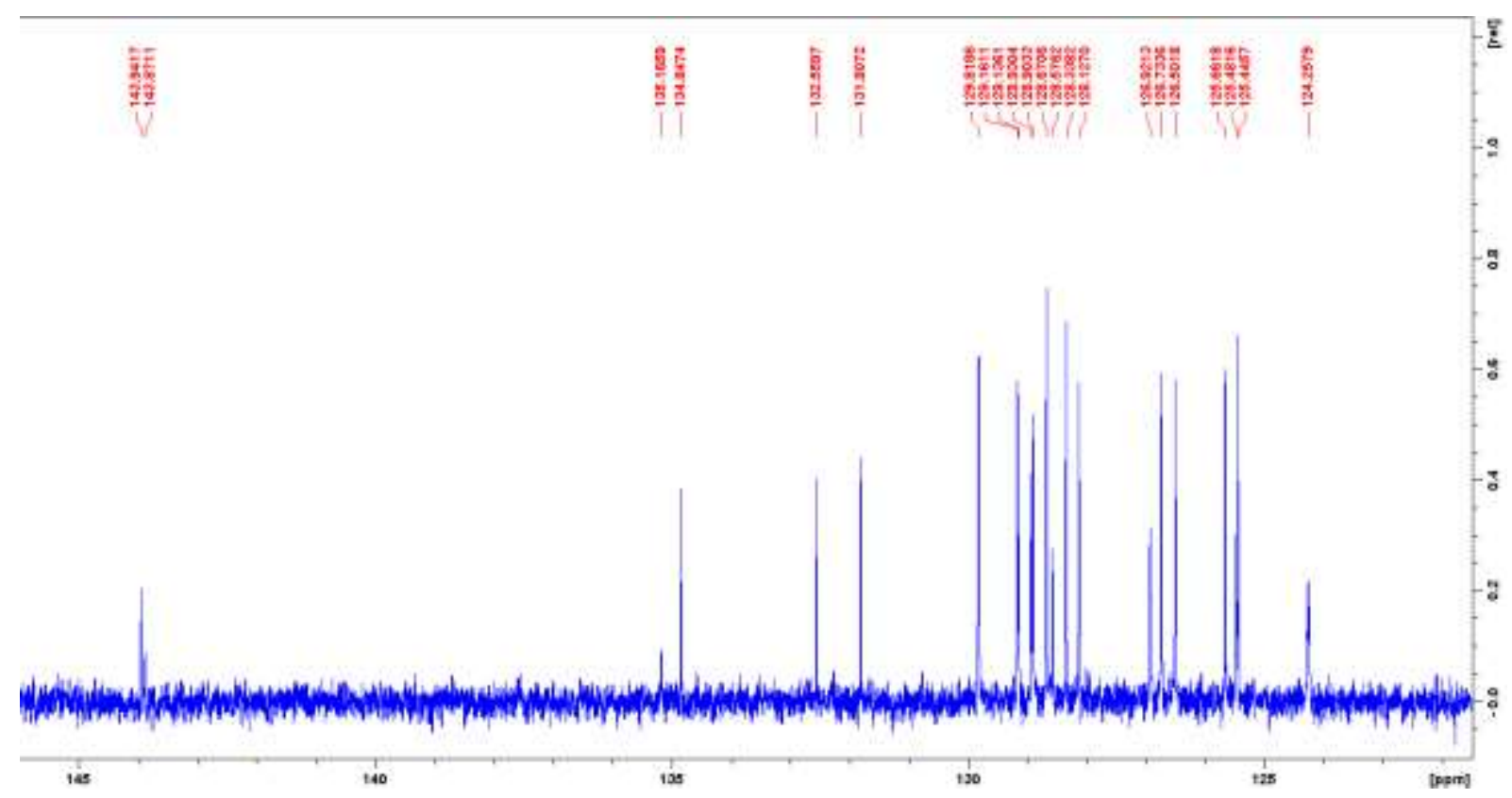

Figure S12: Downfield excerpt of the ${ }^{13} \mathrm{C}\left\{{ }^{1} \mathrm{H}\right\}$ NMR spectrum of 2 in $\mathrm{d}_{8}$-THF.

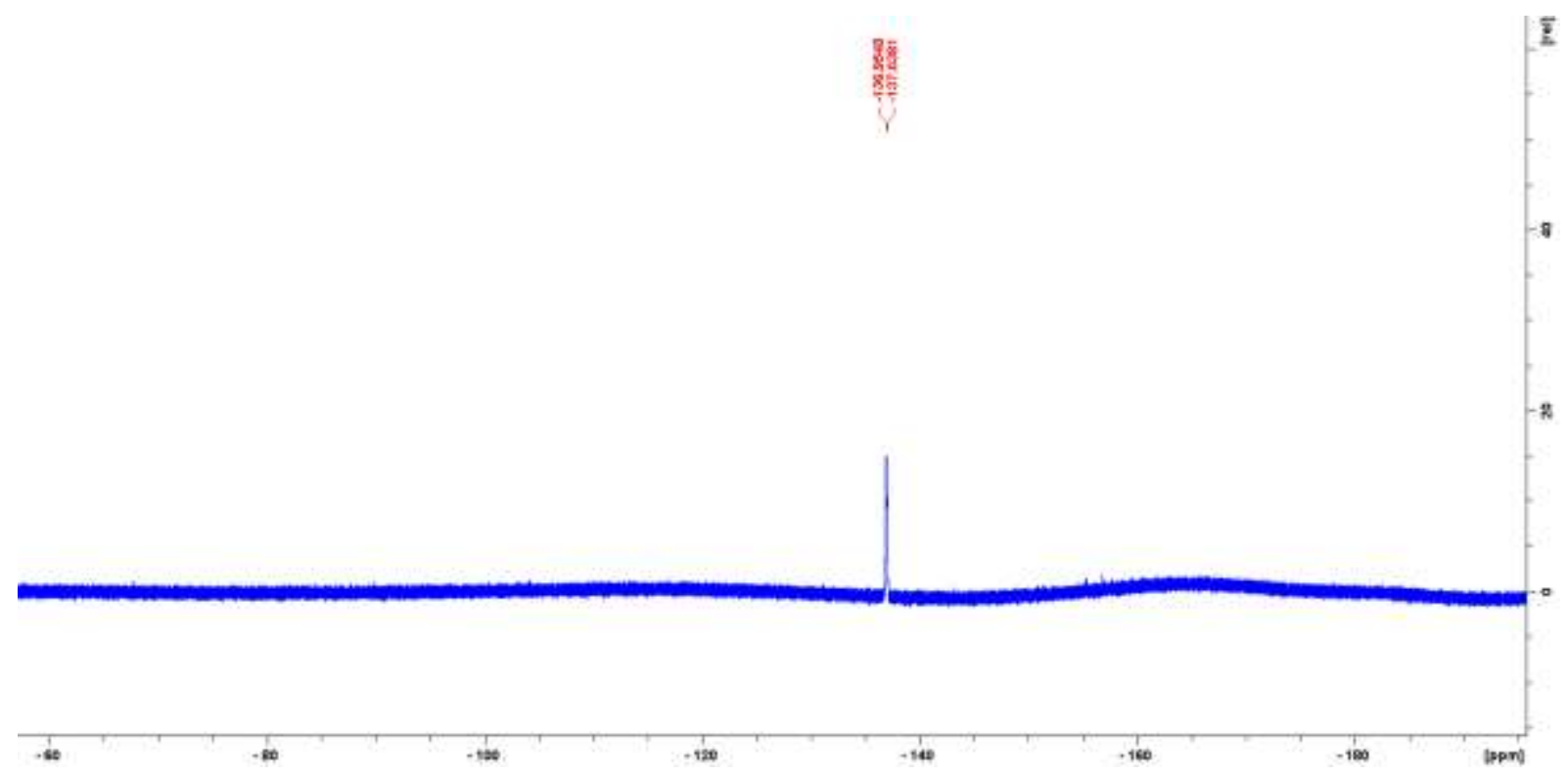

Figure S13: ${ }^{19} \mathrm{~F}\left\{{ }^{1} \mathrm{H}\right\}$ NMR spectrum of 2 in $\mathrm{d}_{8}$-THF. 


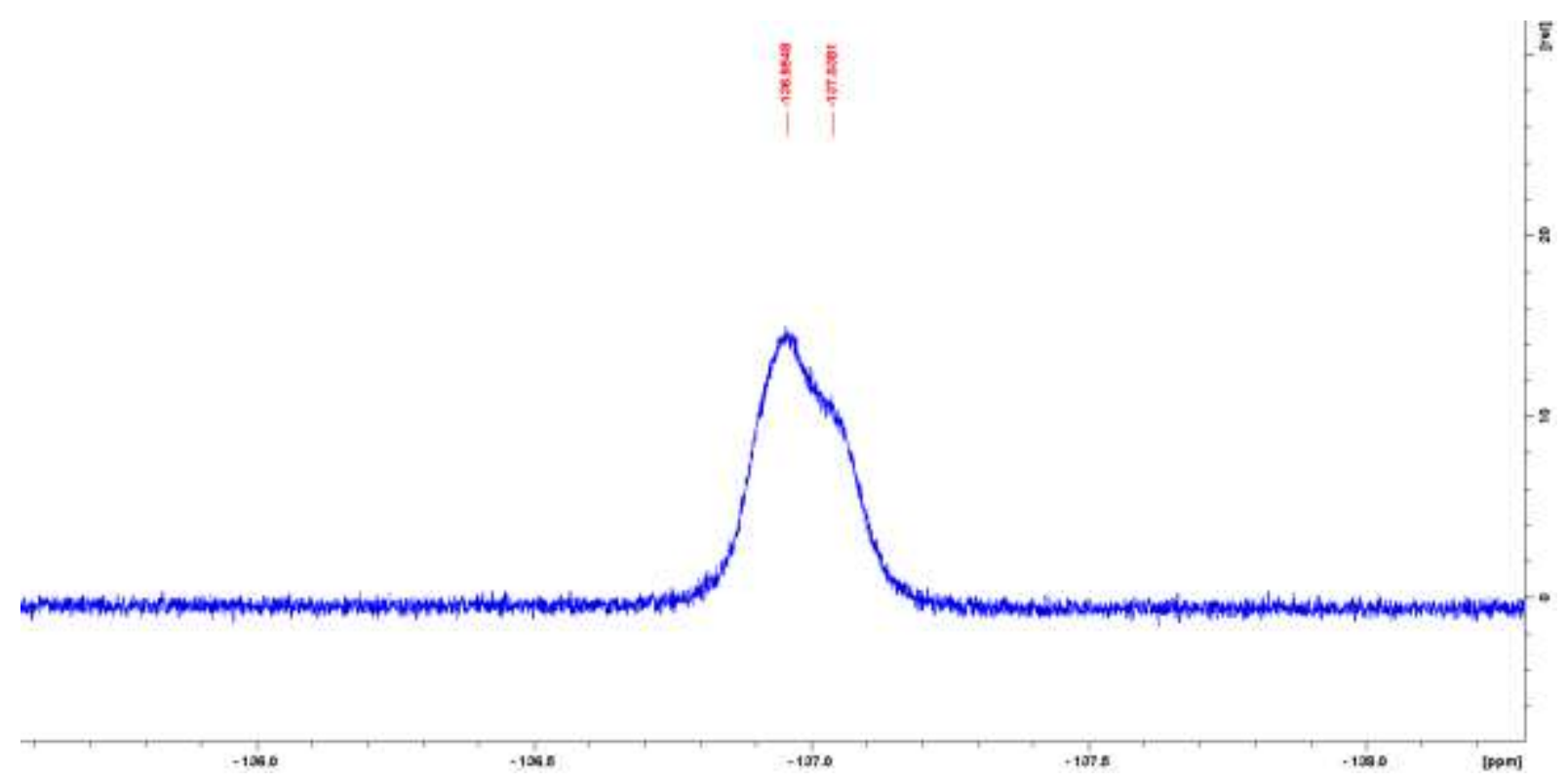

Figure S14: Excerpt of the ${ }^{19} \mathrm{~F}\left\{{ }^{1} \mathrm{H}\right\}$ NMR spectrum of $\mathbf{2}$ in $\mathrm{d}_{8}$-THF.

GBC5-81a-2_011609.10.fid

isolated $\mathrm{B} 2 \mathrm{Mes} 2 \mathrm{Cl} 2+\mathrm{xTMSN} 3$

c6d6

APROTON C6D6 \{D:|Topspin21\} User 1
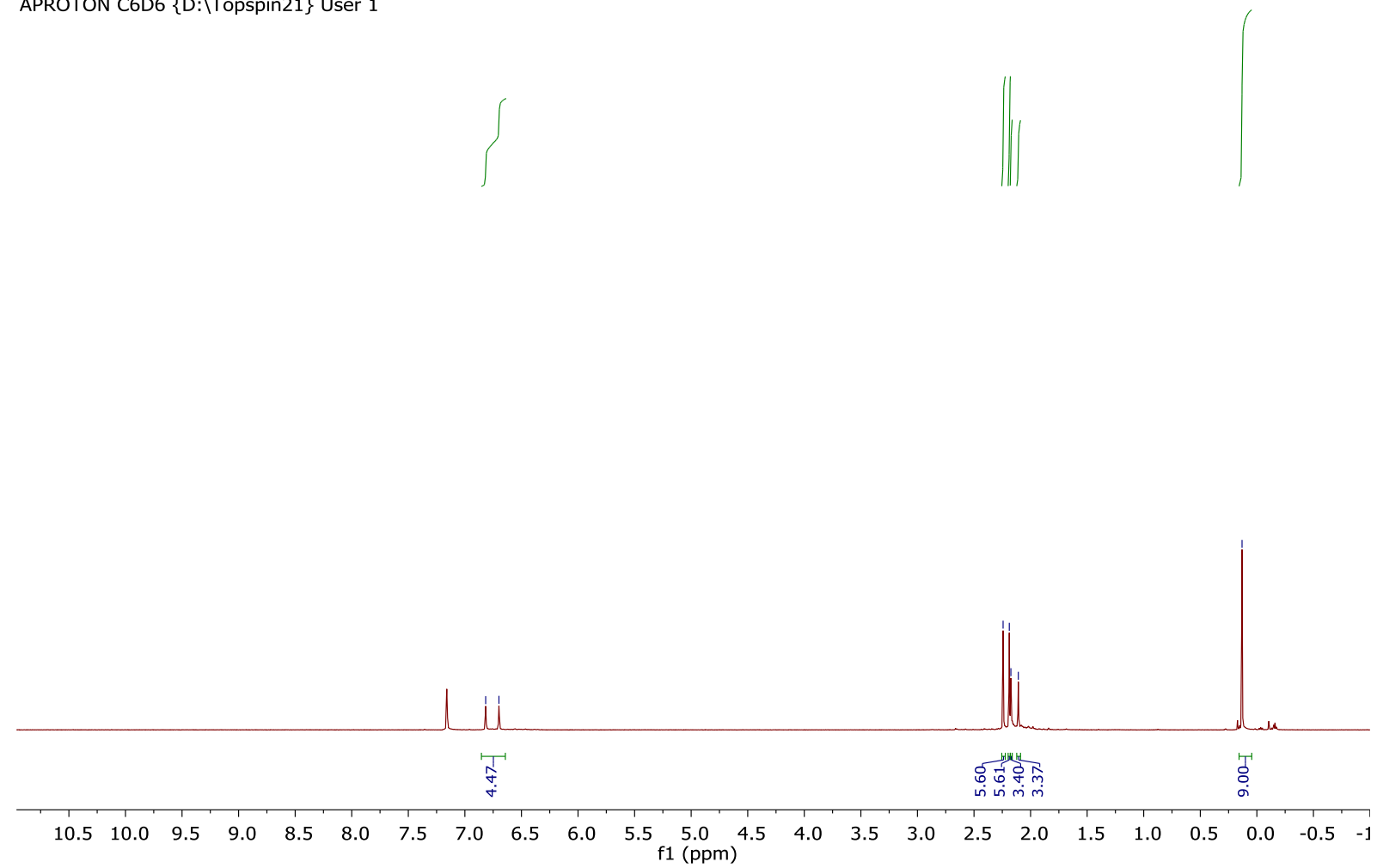

Figure S15: ${ }^{1} \mathrm{H}$ NMR spectrum of $\mathbf{6 a}$ in $\mathrm{C}_{6} \mathrm{D}_{6}$. 
A11BZG C6D6 \{D:|Topspin21\} User 1

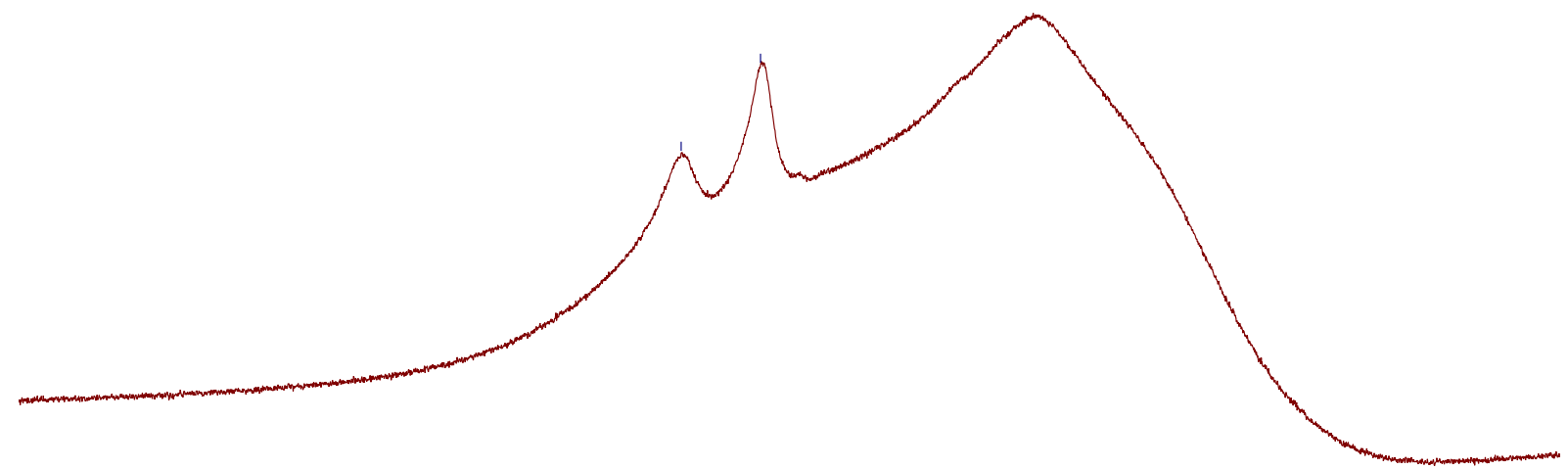

$40 \quad 30$

20

$\begin{array}{llll}10 & 0 & -10 & -20\end{array}$

$\begin{array}{llll}-30 & -40 & -50 & -60\end{array}$

Figure S16: ${ }^{11} \mathrm{~B}$ NMR spectrum of $\mathbf{6 a}$ in $\mathrm{C}_{6} \mathrm{D}_{6}$.

GBC5-81a-2_011609.14.fid

isolated $\mathrm{B} 2 \mathrm{Mes} 2 \mathrm{Cl} 2+\mathrm{xTMSN} 3$

c6d6

AC13CPD C6D6 \{D:|Topspin21\} User 1

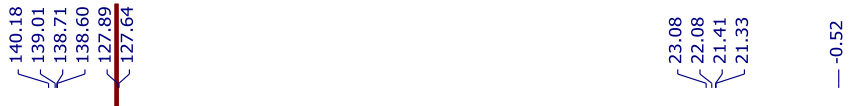

$\begin{array}{lllllllllllllllllllllllllllllll}50 & 240 & 230 & 220 & 210 & 200 & 190 & 180 & 170 & 160 & 150 & 140 & 130 & 120 & 110 & 100 & 90 & 80 & 70 & 60 & 50 & 40 & 30 & 20 & 10 & 0 & -10 & -20\end{array}$ 1 (ppm)

Figure S17: ${ }^{13} \mathrm{C}\left\{{ }^{1} \mathrm{H}\right\}$ NMR spectrum of $\mathbf{6 a}$ in $\mathrm{C}_{6} \mathrm{D}_{6}$. 


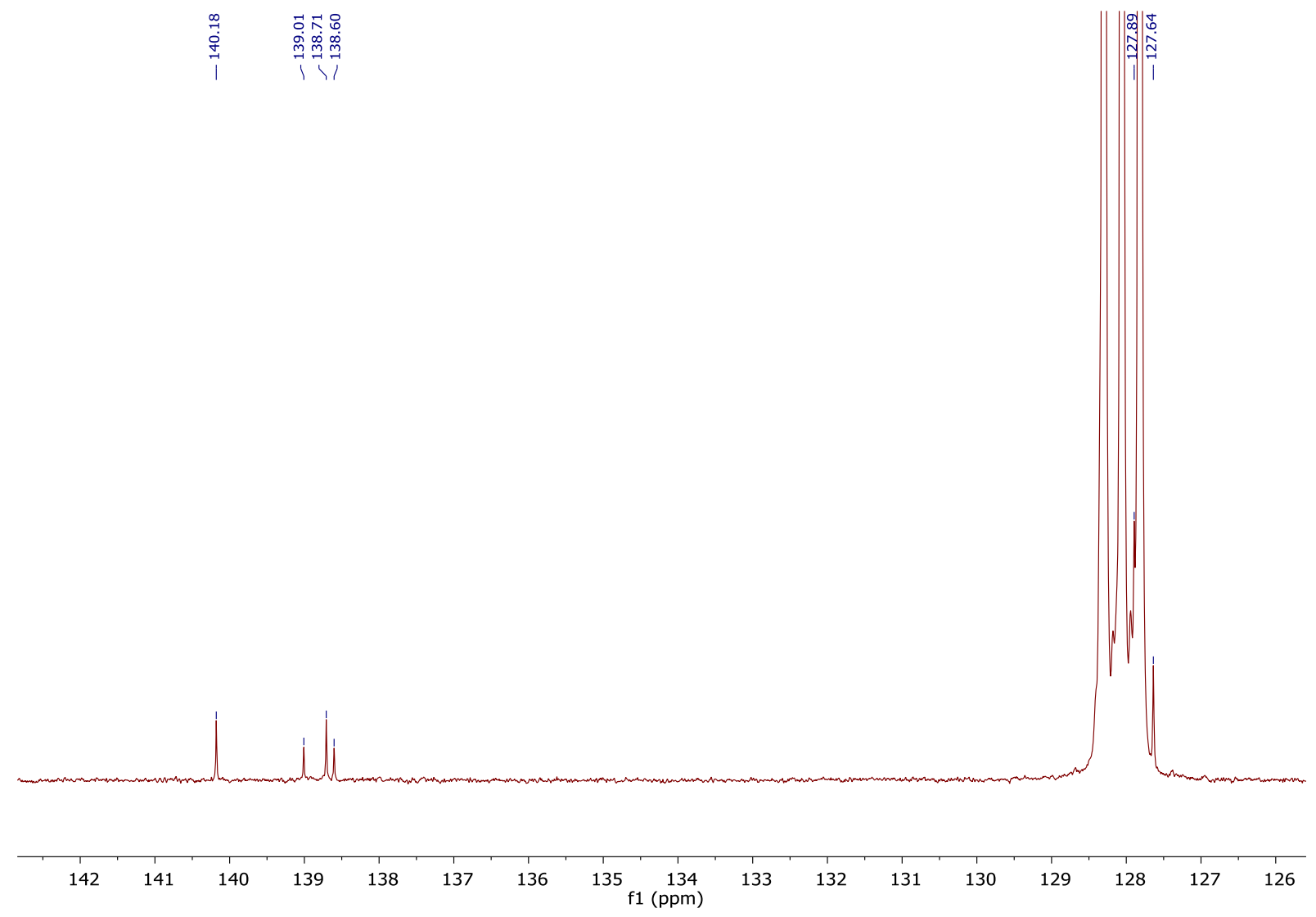

Figure $\mathrm{S} 18:{ }^{13} \mathrm{C}\left\{{ }^{1} \mathrm{H}\right\}$ NMR spectrum of $6 \mathbf{6}$ in $\mathrm{C}_{6} \mathrm{D}_{6}$ (aromatic region). 


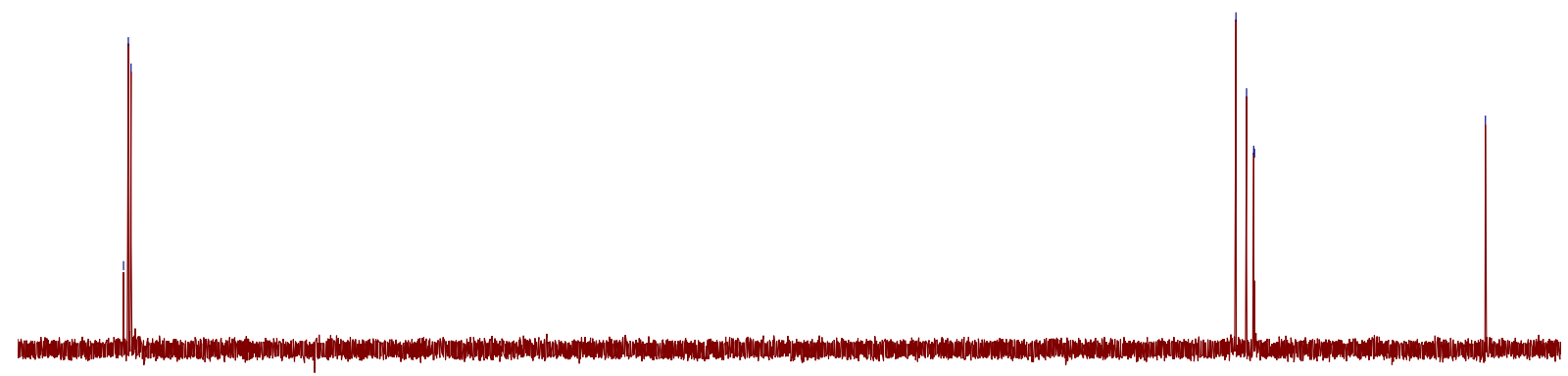

$\begin{array}{lllllllllllllllllllllllllllll}135 & 130 & 125 & 120 & 115 & 110 & 105 & 100 & 95 & 90 & 85 & 80 & 75 & 70 & 65 & 60 & 55 & 50 & 45 & 40 & 35 & 30 & 25 & 20 & 15 & 10 & 5 & 0 & -5\end{array}$

Figure S19: ${ }^{13} \mathrm{C}$ DEPT135 NMR spectrum of $\mathbf{6 a}$ in $\mathrm{C}_{6} \mathrm{D}_{6}$. The signal at 128.35 is assigned to $\mathrm{C}_{6} \mathrm{H}_{6}$.

GBC5-81a-2_011609.14.fid

isolated B2Mes2CI2 + XTMSN3

c6d6

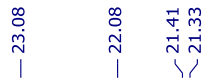

AC13CPD C6D6 \{D:|Topspin21\} User 1

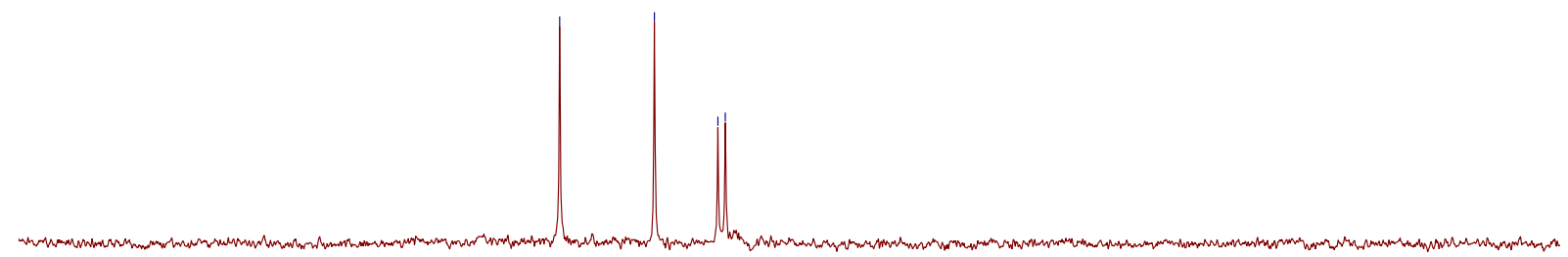

Figure $\mathrm{S} 20:{ }^{13} \mathrm{C}\left\{{ }^{1} \mathrm{H}\right\}$ NMR spectrum of $\mathbf{6 a}$ in $\mathrm{C}_{6} \mathrm{D}_{6}$ (aliphatic region). 


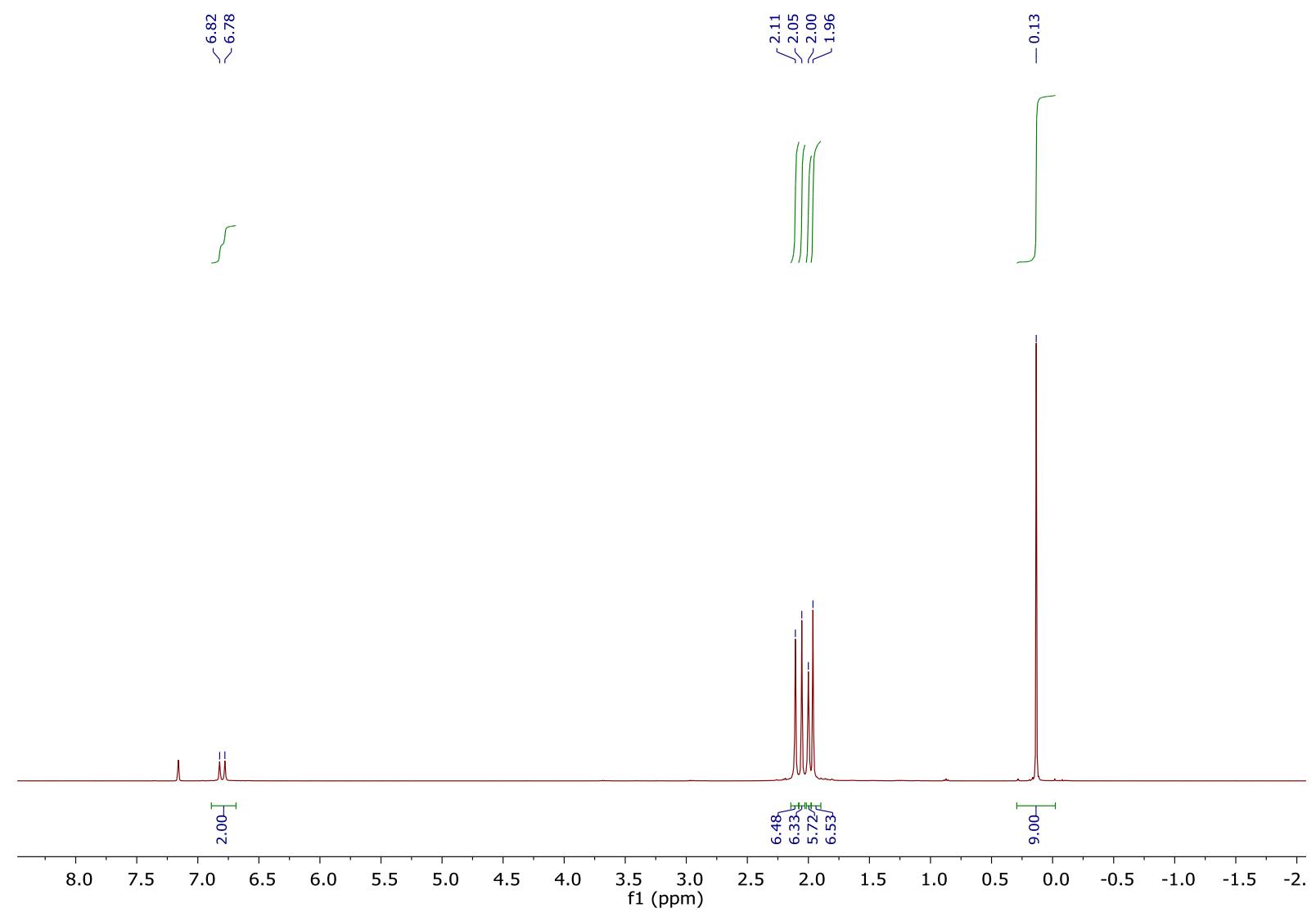

Figure S21: ${ }^{1} \mathrm{H}$ NMR spectrum of $\mathbf{6 b}$ in $\mathrm{C}_{6} \mathrm{D}_{6}$. 


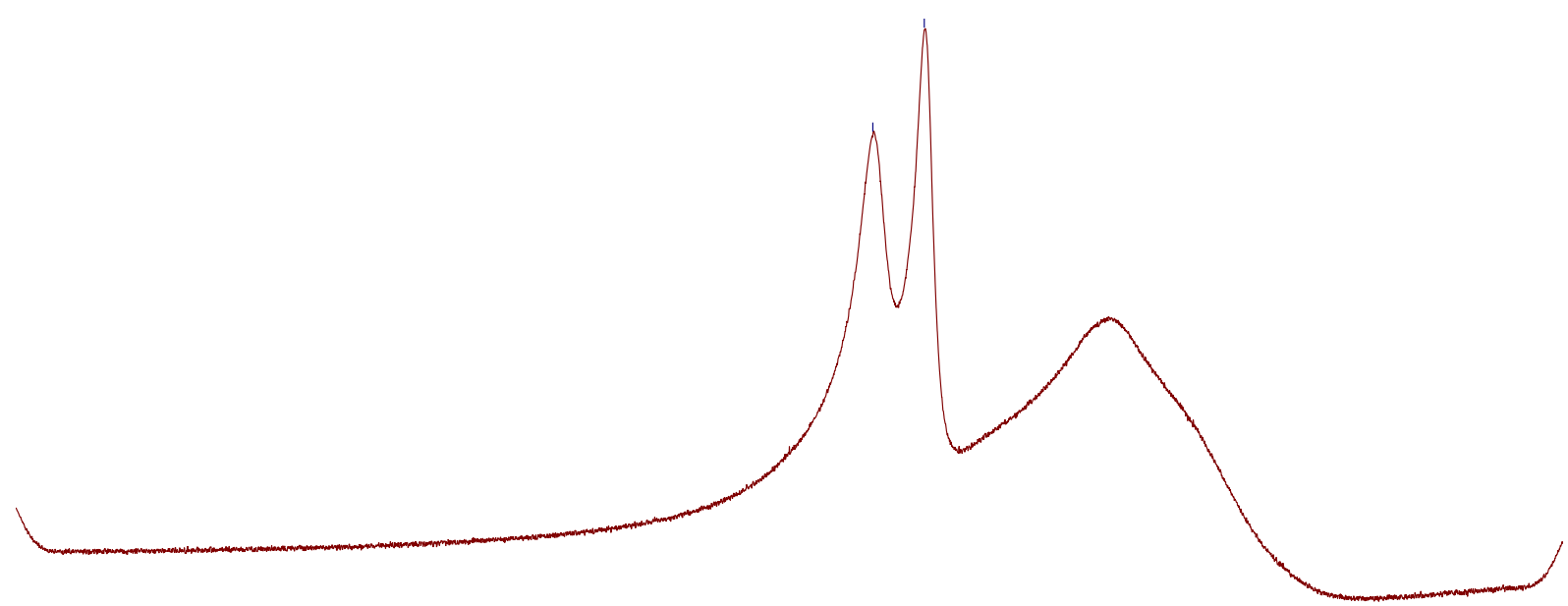

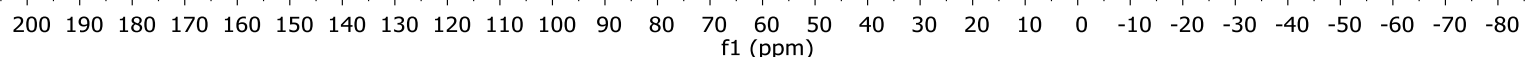

Figure S22: ${ }^{11} \mathrm{~B}$ NMR spectrum of $6 \mathbf{b}$ in $\mathrm{C}_{6} \mathrm{D}_{6}$.

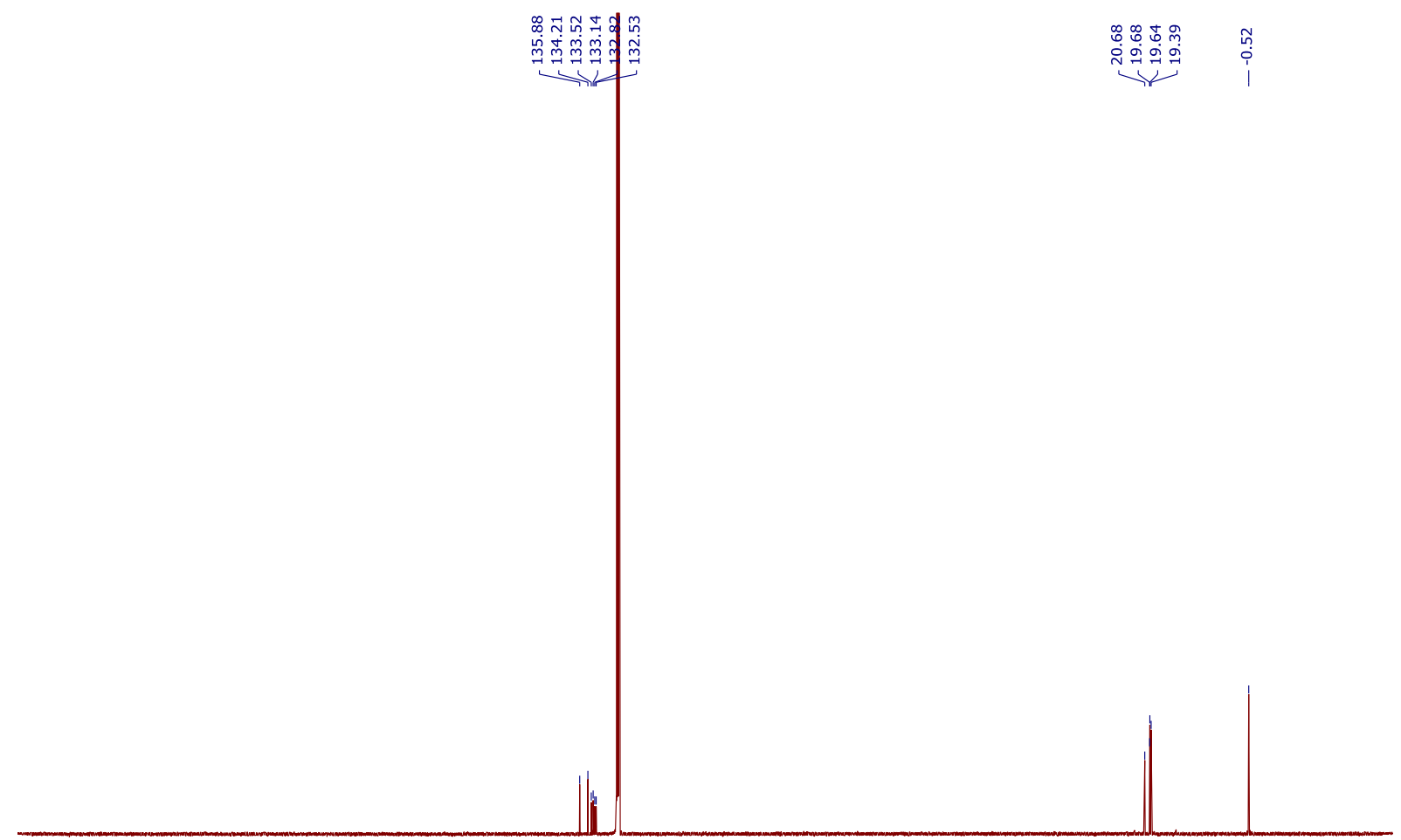

$\begin{array}{lllllllllllllllllllllllllllll}50 & 240 & 230 & 220 & 210 & 200 & 190 & 180 & 170 & 160 & 150 & 140 & 130 & 120 & 110 & 100 & 90 & 80 & 70 & 60 & 50 & 40 & 30 & 20 & 10 & 0 & -10 & -20\end{array}$

Figure S23: ${ }^{13} \mathrm{C}\left\{{ }^{1} \mathrm{H}\right\}$ NMR spectrum of $\mathbf{6 b}$ in $\mathrm{C}_{6} \mathrm{D}_{6}$. 


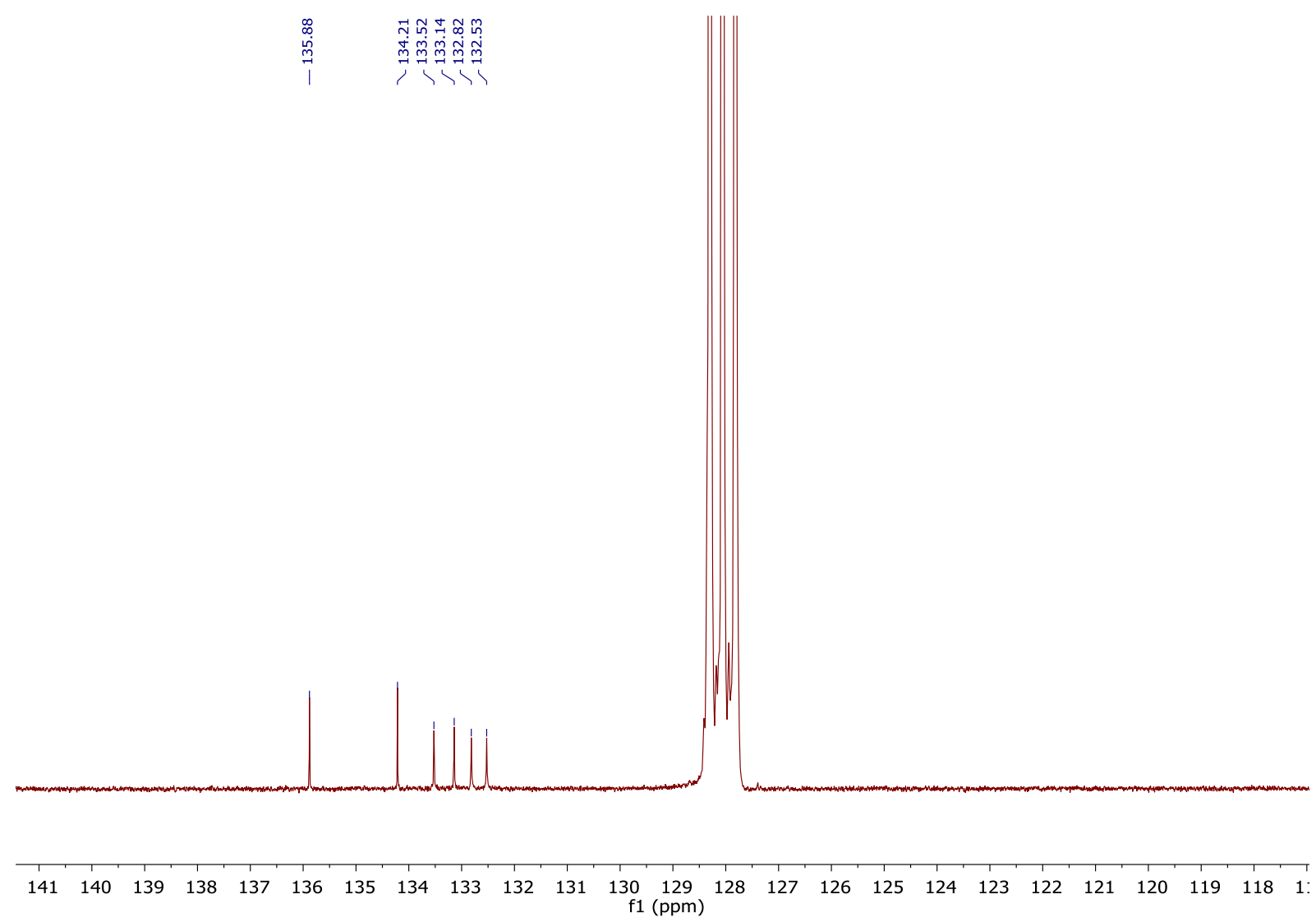

Figure S24: ${ }^{13} \mathrm{C}\left\{{ }^{1} \mathrm{H}\right\}$ NMR spectrum of $6 \mathbf{b}$ in $\mathrm{C}_{6} \mathrm{D}_{6}$ (aromatic region).

踏

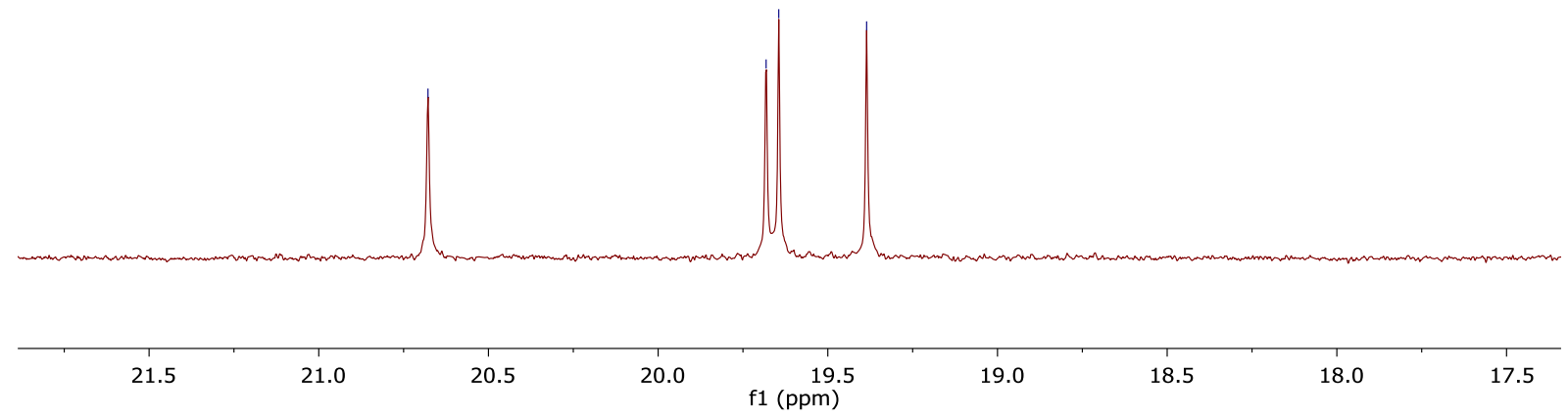

Figure $S 25:{ }^{13} \mathrm{C}\left\{{ }^{1} \mathrm{H}\right\}$ NMR spectrum of $6 \mathbf{b}$ in $\mathrm{C}_{6} \mathrm{D}_{6}$ (aliphatic region). 
$\begin{array}{lll}0 & 1 \\ 0 & 4 & 1\end{array}$
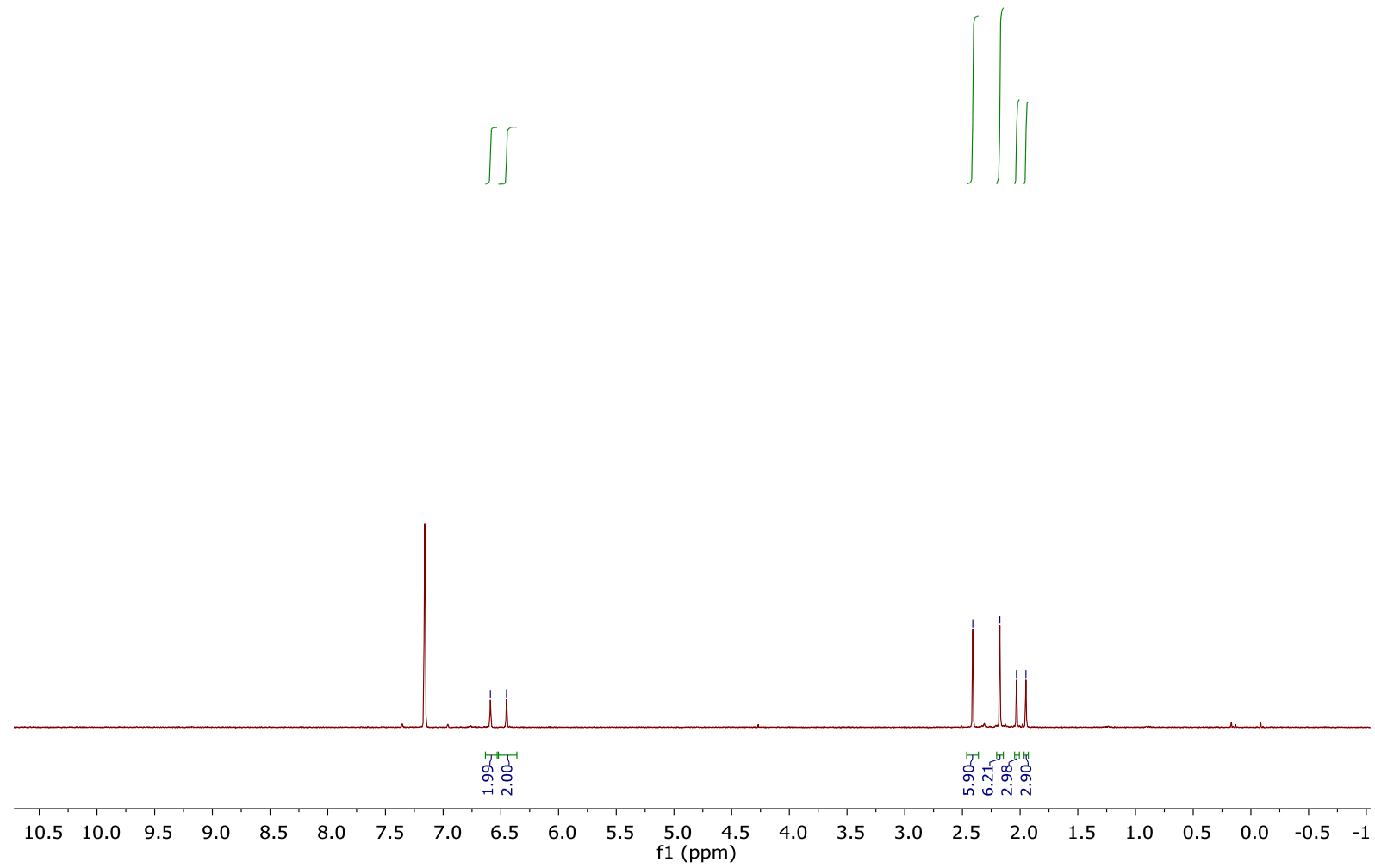

Figure S26: ${ }^{1} \mathrm{H}$ NMR spectrum of 7 in $\mathrm{C}_{6} \mathrm{D}_{6}$.

GBC5-158b-4_602309.13.fid

A11BZG C6D6 \{D:|Topspin21\} User 60

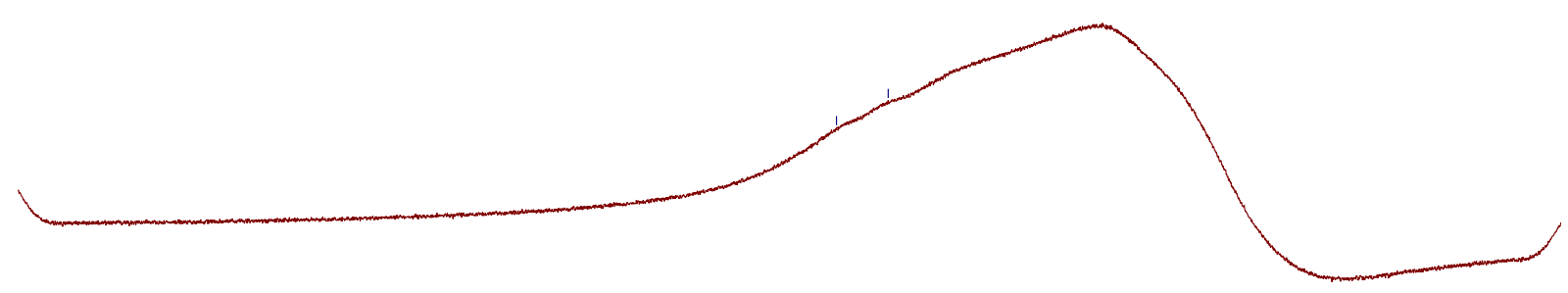

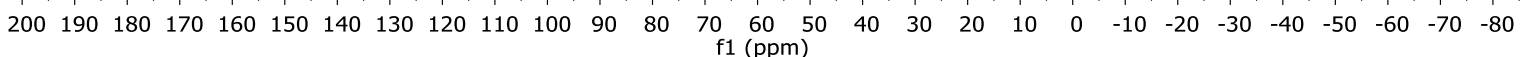

Figure S27: ${ }^{11} \mathrm{~B}$ NMR spectrum of isolated 7 in $\mathrm{C}_{6} \mathrm{D}_{6} .7$ is almost impossible to detect in the concentrations obtained. The very faint features annotated here are however inconsistent with the 
normal ${ }^{11} \mathrm{~B}$ tube/instrument borosilicate background and consistent with the expected chemical shifts of a BMes $\left(\mathrm{N}_{3}\right)$-subsituted diazadiboretidine (B3lyp/6-311+g(d,p) predicted ${ }^{11} \mathrm{~B}$ NMR shifts: 49.2 and $39.6 \mathrm{ppm})$.

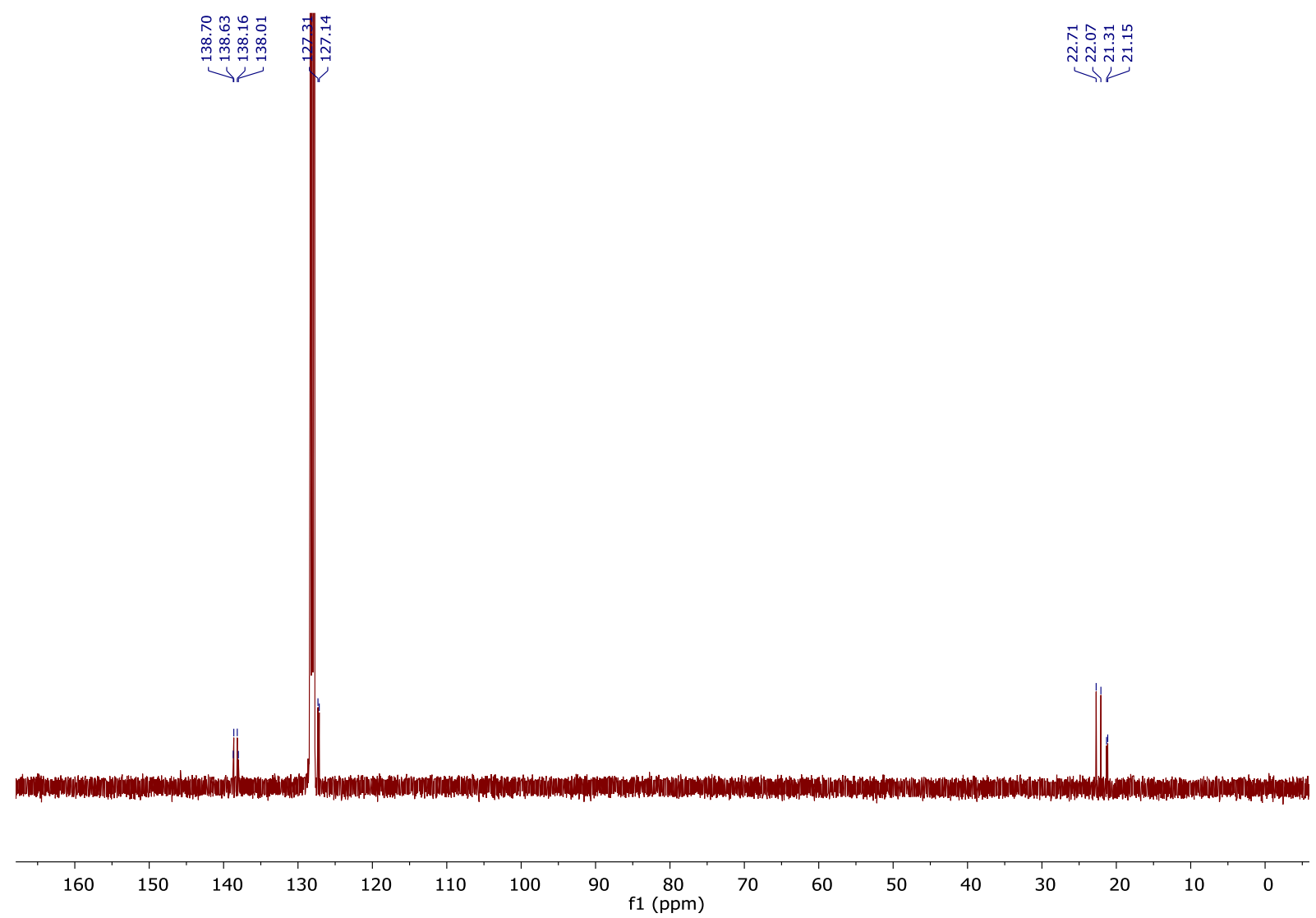

Figure S28: ${ }^{13} \mathrm{C}\left\{{ }^{1} \mathrm{H}\right\}$ NMR spectrum of 7 in $\mathrm{C}_{6} \mathrm{D}_{6}$. 
GBC5-158b-4_602309. 906 fid

AC13CPD C6D6 \{

1,11
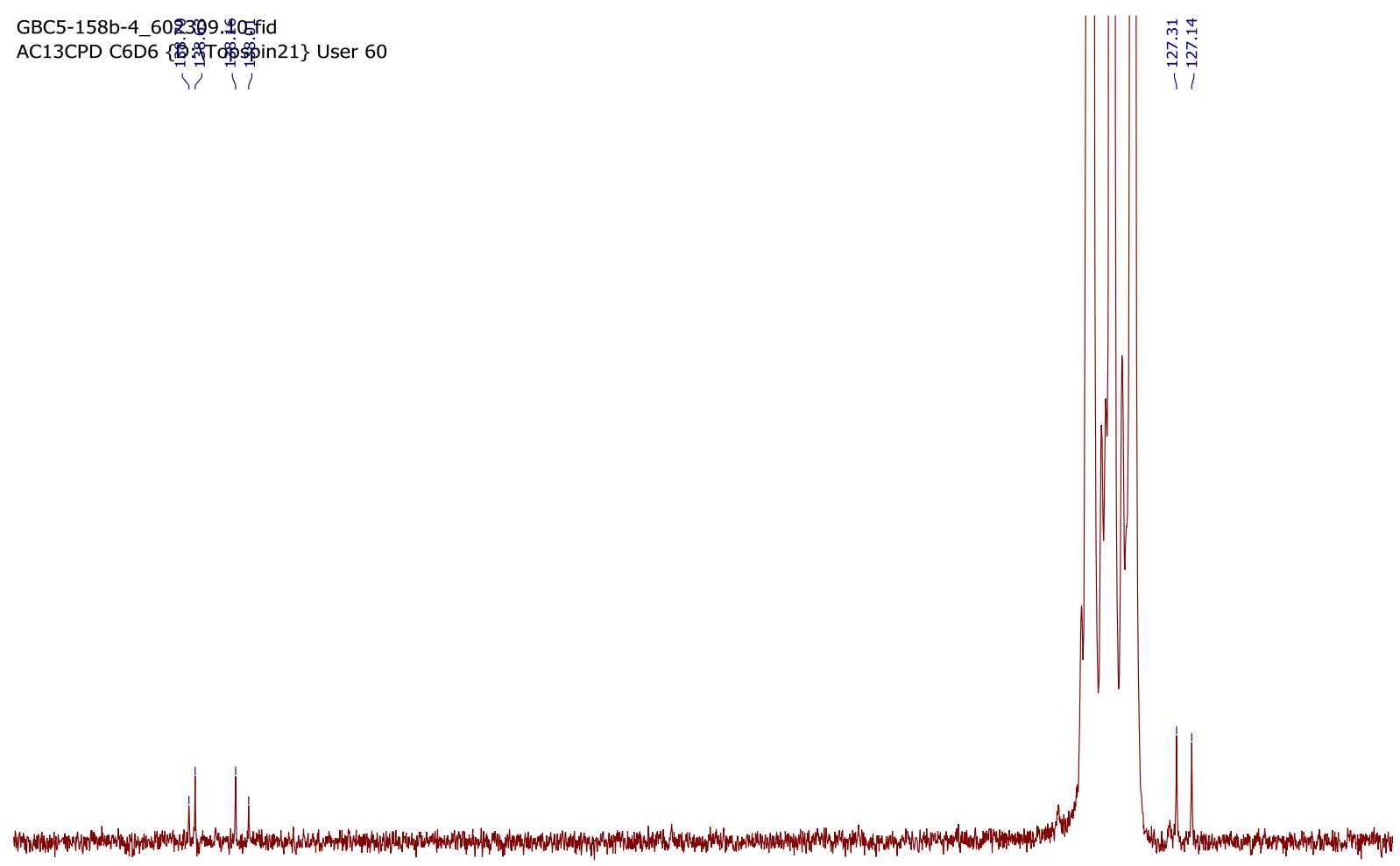

\begin{tabular}{|c|c|c|c|c|c|c|c|c|c|c|c|c|c|c|c|}
\hline 140 & 139 & 138 & 137 & 136 & 135 & 134 & $\begin{array}{l}133 \\
\text { f1 (ppm) }\end{array}$ & 132 & 131 & 130 & 129 & 128 & 127 & 126 & 125 \\
\hline
\end{tabular}

Figure S29: ${ }^{13} \mathrm{C}\left\{{ }^{1} \mathrm{H}\right\}$ NMR spectrum of 7 in $\mathrm{C}_{6} \mathrm{D}_{6}$ (aromatic region).

GBC5-158b-4_602309.10.fid

AC13CPD C6D6 \{D:|Topspin21\} User 60

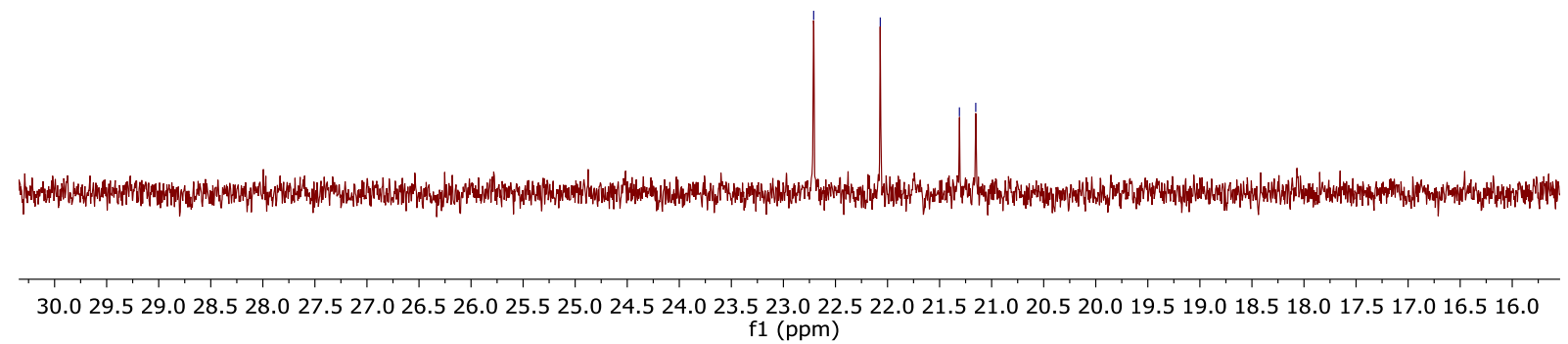

Figure S30: ${ }^{13} \mathrm{C}\left\{{ }^{1} \mathrm{H}\right\}$ NMR spectrum of 7 in $\mathrm{C}_{6} \mathrm{D}_{6}$ (aliphatic region). 


\section{$\underline{\text { UV/Vis Spectroscopy }}$}

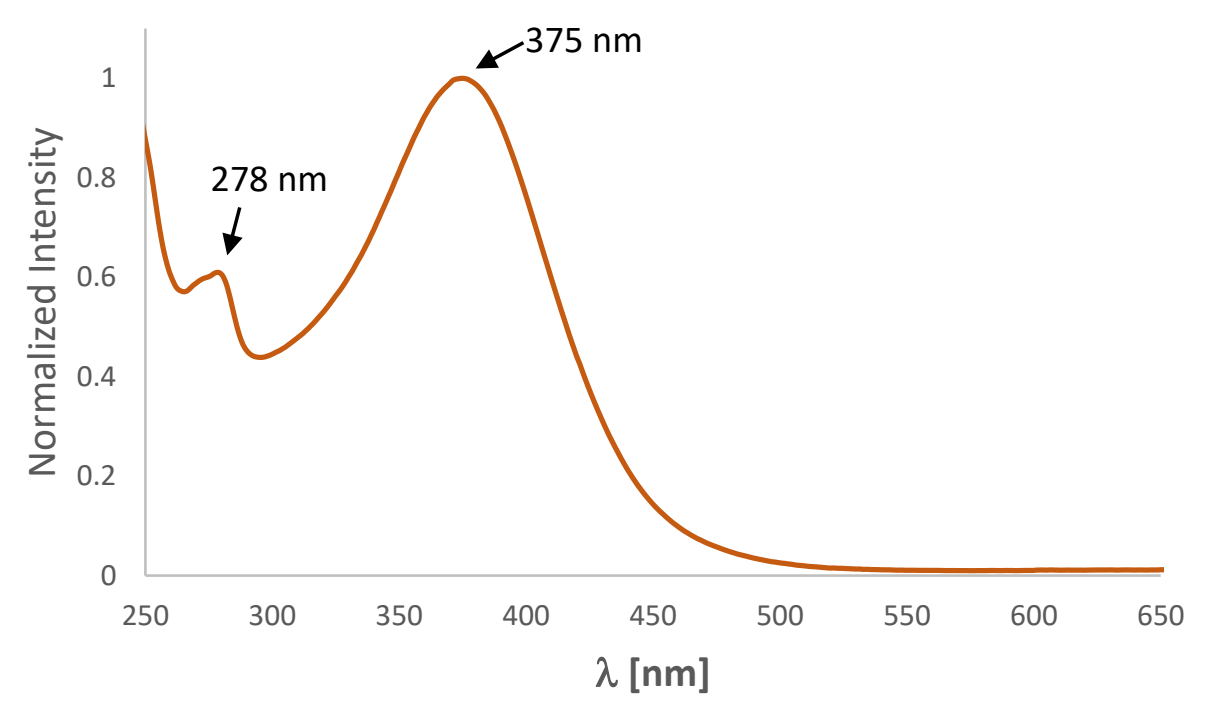

Figure S31: UV/Vis absorption spectrum of 1a in acetonitrile.

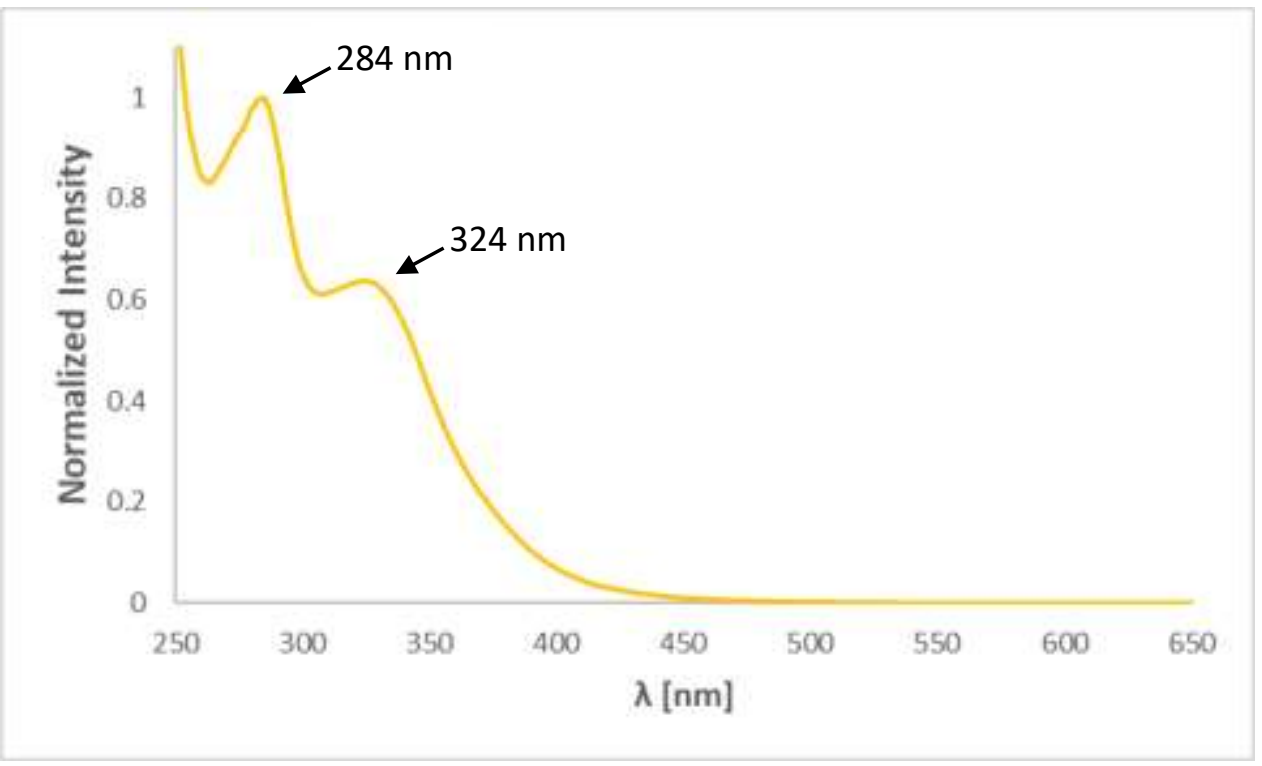

Figure S32: UV/Vis absorption spectrum of $\mathbf{1 b}$ in acetonitrile. 


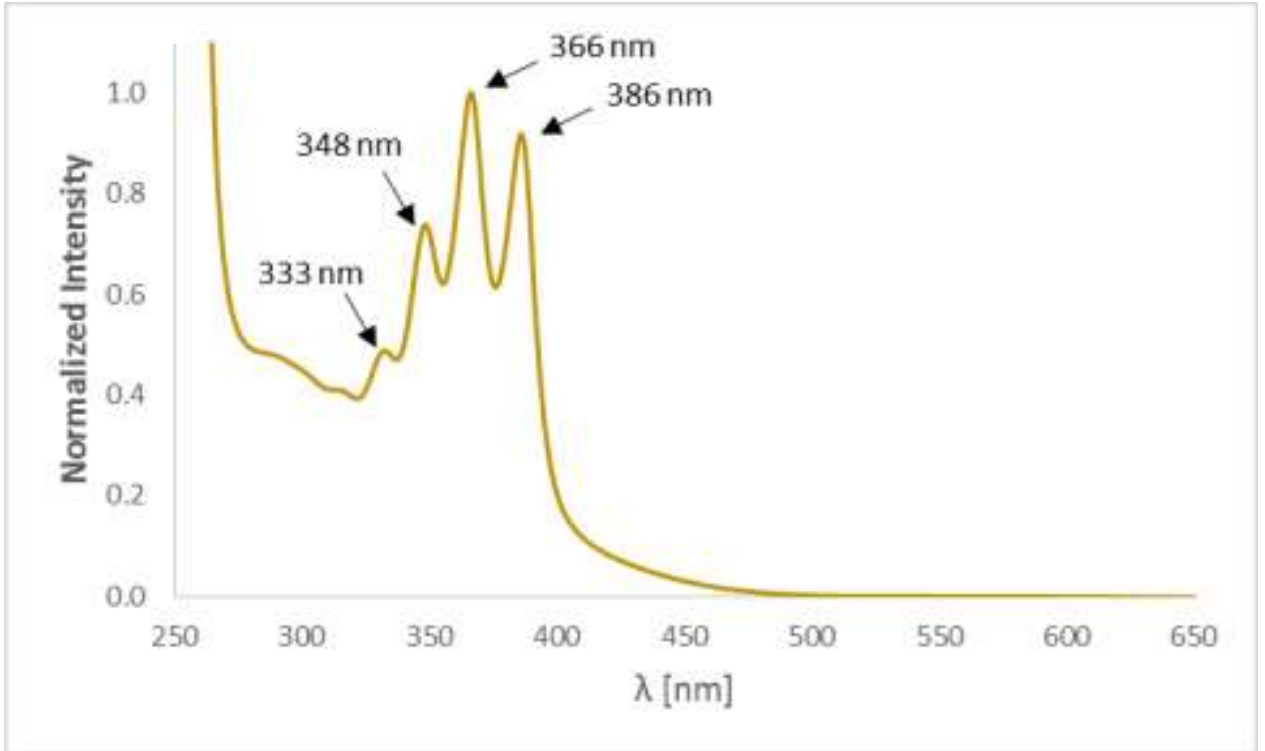

Figure S33: UV/Vis absorption spectrum of $\mathbf{2}$ in acetonitrile. 


\section{$\underline{\text { IR Spectroscopy }}$}

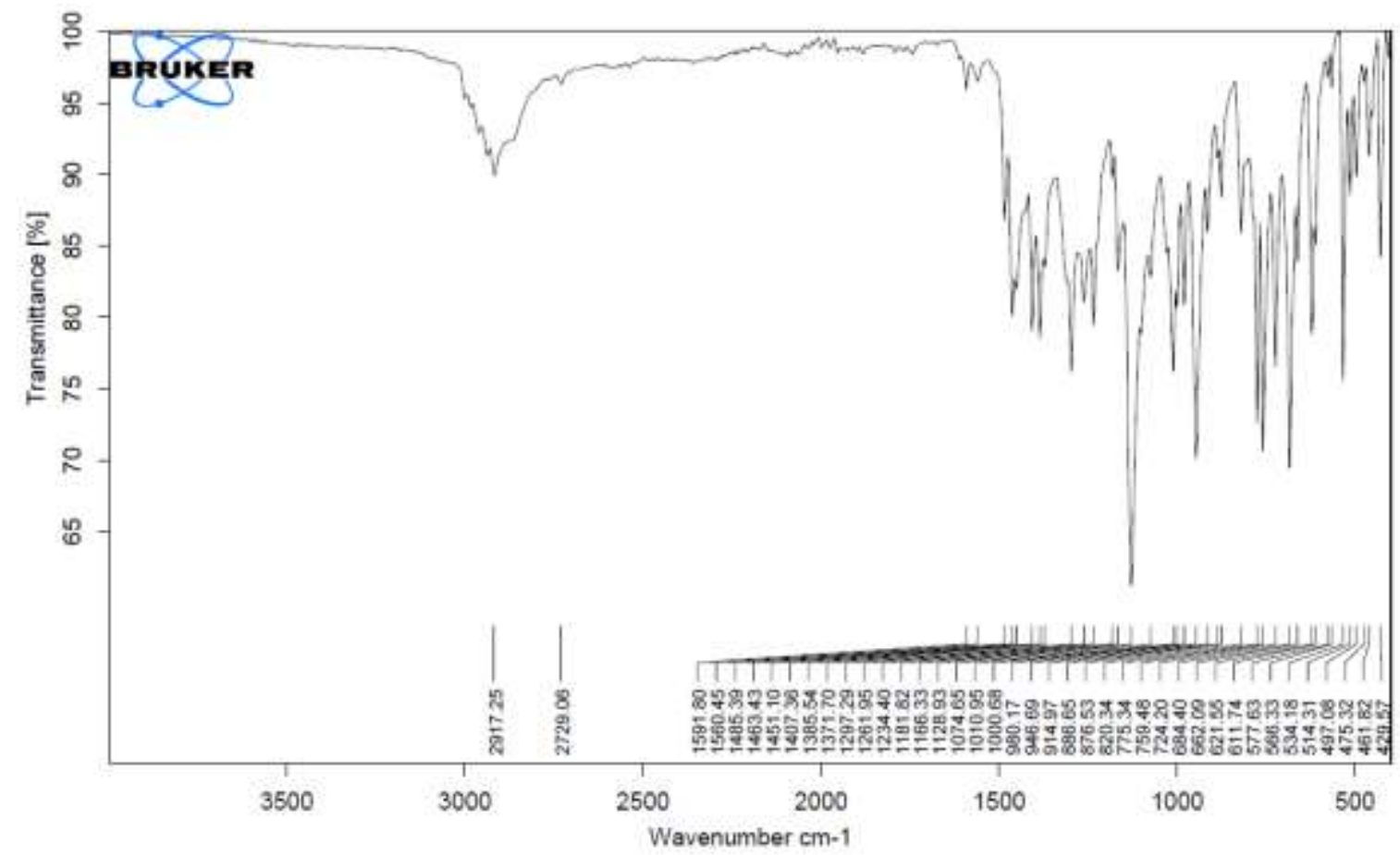

Figure S34: Solid-state IR spectrum of 1a.

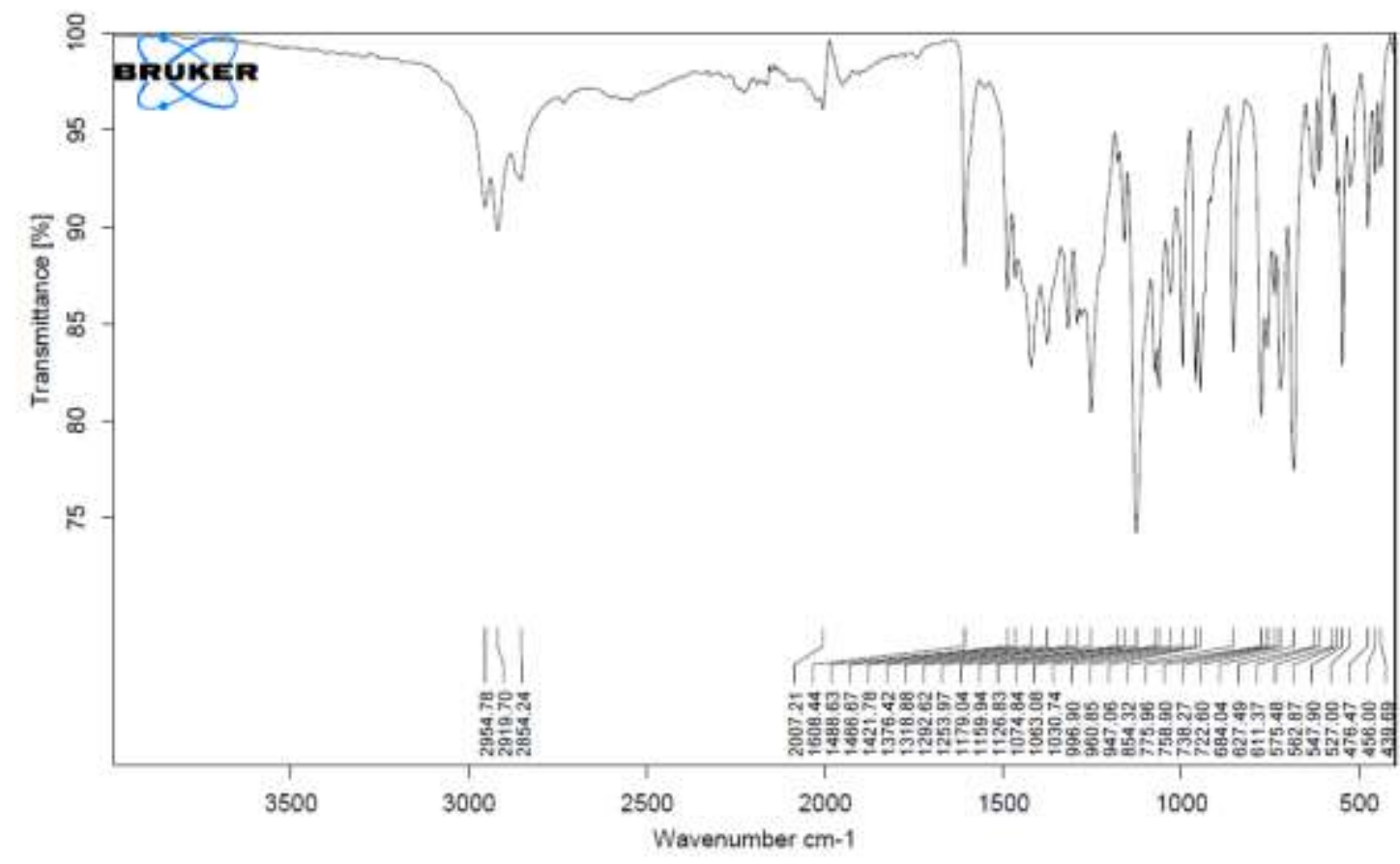

Figure S35: Solid-state IR spectrum of $\mathbf{1 b}$. 


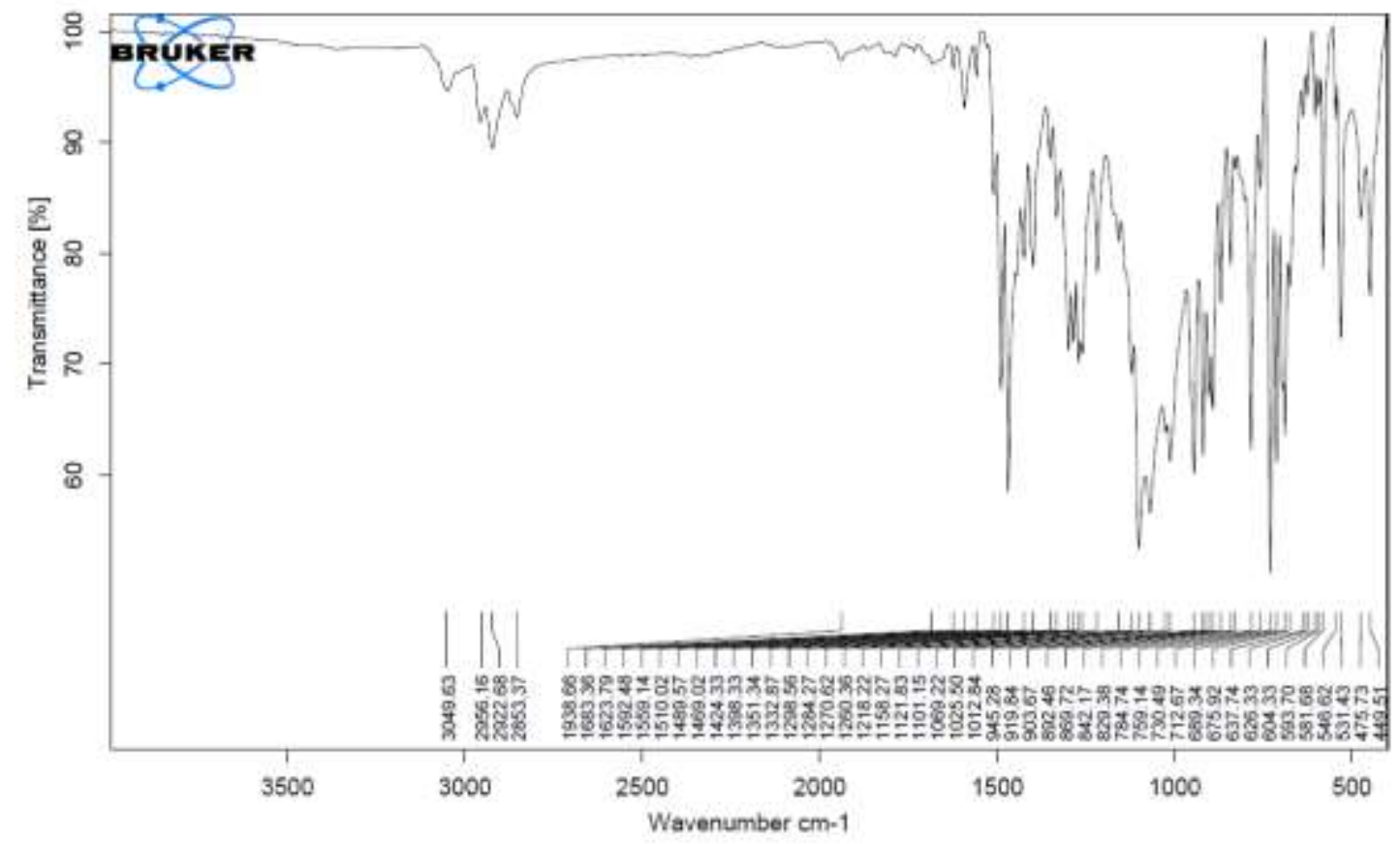

Figure S36: Solid-state IR spectrum of 2. 


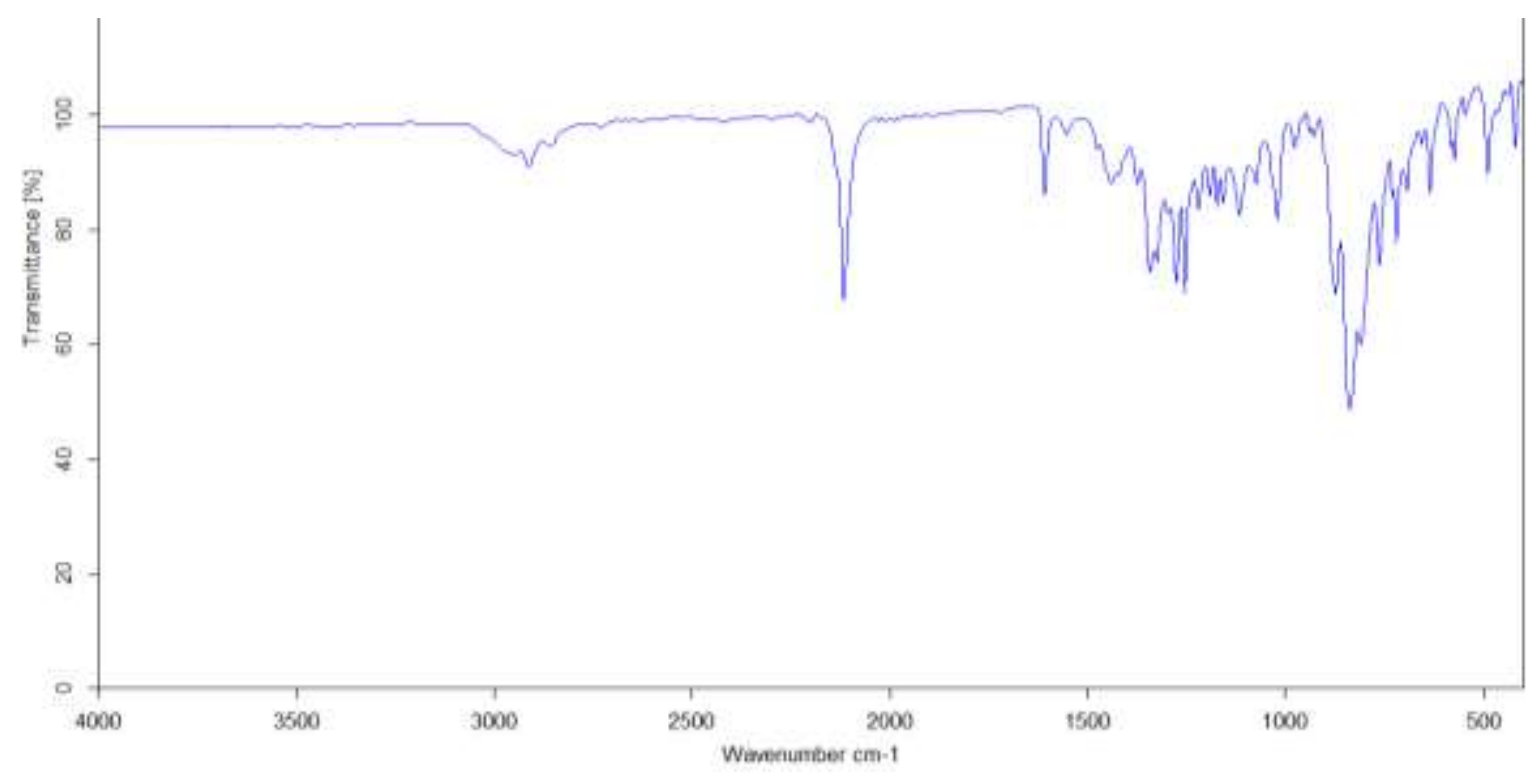

Figure S37: Solid-state IR spectrum of $\mathbf{6 a}$.

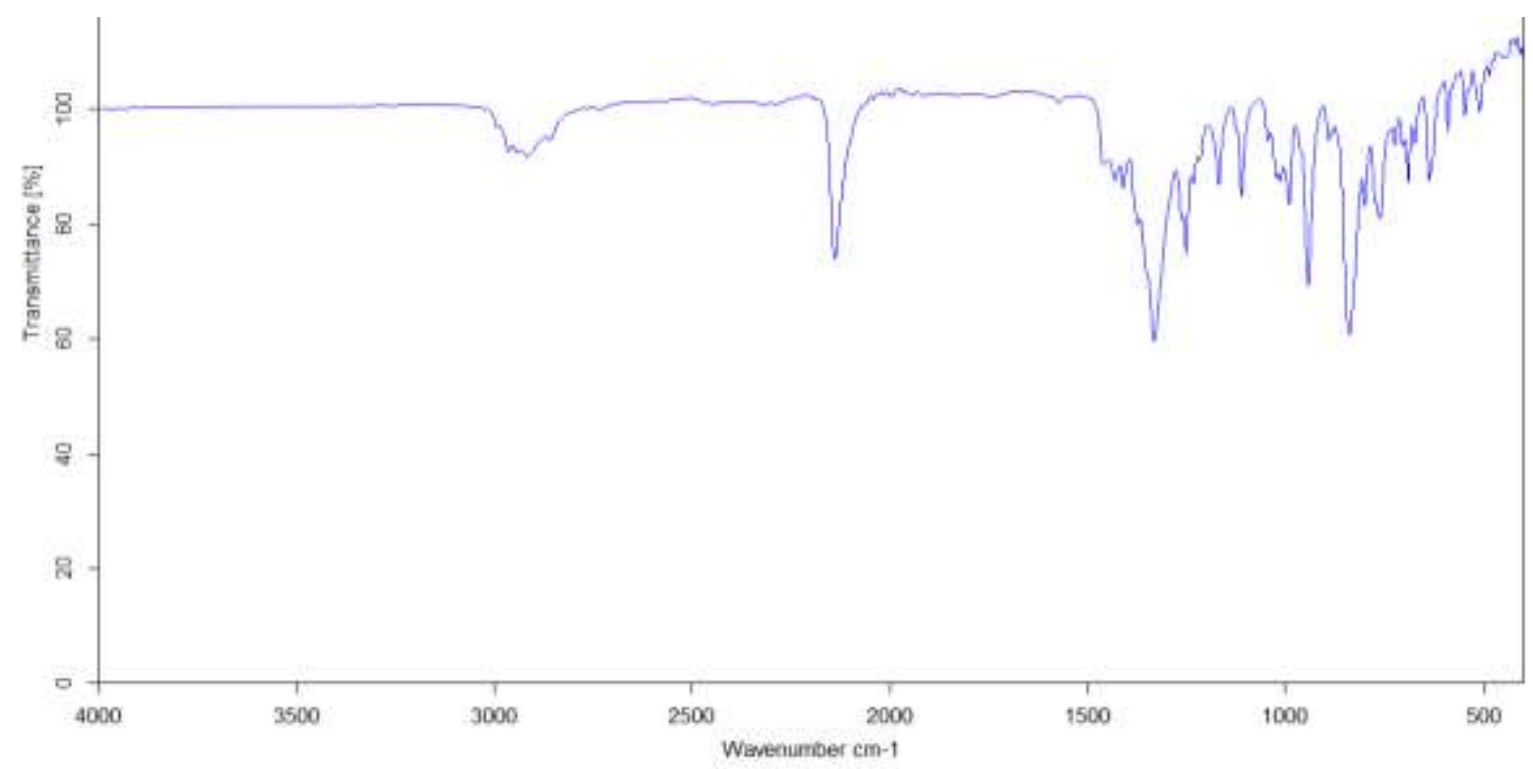

Figure S38: Solid-state IR spectrum of $\mathbf{6 b}$. 


\section{Cyclic Voltammetry}

Cyclic voltammetry experiments were performed using a Gamry Instruments Reference 600 potentiostat. A standard three-electrode cell configuration was employed using a platinum disk working electrode, a platinum wire counter-electrode, and a silver wire, separated by a Vycor tip, serving as the reference electrode. Tetra- $n$-butylammonium hexafluorophosphate $\left(\left[n \mathrm{Bu}_{4} \mathrm{~N}\right]\left[\mathrm{PF}_{6}\right]\right)$ was employed as the supporting electrolyte. Compensation for resistive losses ( $i R$ drop) was employed for all measurements. Formal redox potentials are referenced to the ferrocene/ferrocenium $\left(\left[\mathrm{Cp}_{2} \mathrm{Fe}\right]^{+/ 0}\right)$ redox couple.

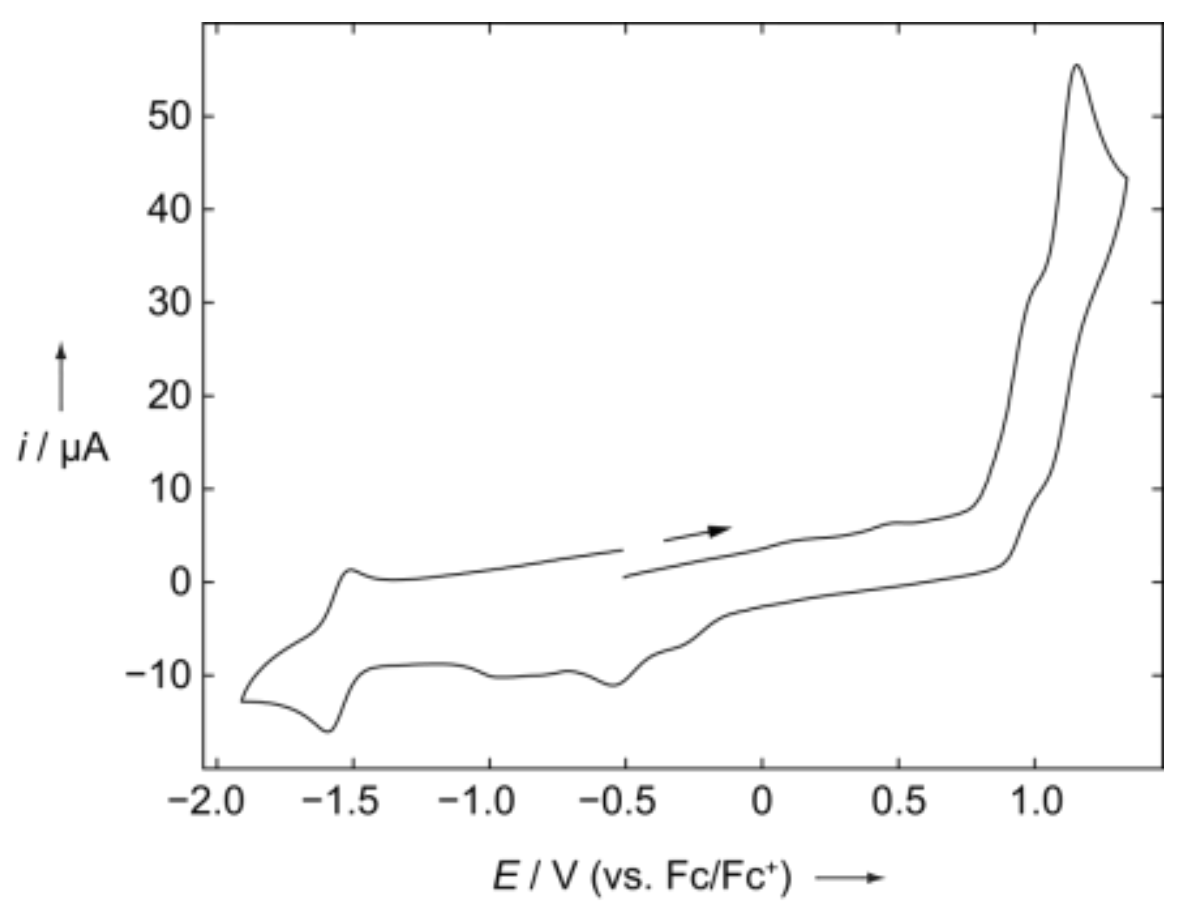

Figure S39: Cyclic voltammogram of 2 in $\mathrm{CH}_{3} \mathrm{CN} / 0.1 \mathrm{M}\left[n \mathrm{Bu}_{4} \mathrm{~N}\right]\left[\mathrm{PF}_{6}\right]$ measured at $250 \mathrm{mV} \mathrm{s}^{-1}$. Formal potentials: $\mathrm{E}_{1 / 2}=-1.55 \mathrm{~V}, \mathrm{E}_{\mathrm{pa}}=+1.00 \mathrm{~V}$ and $\mathrm{E}_{\mathrm{pa}}=+1.15 \mathrm{~V}$ (relative to the $\mathrm{Fc} / \mathrm{Fc}^{+}$couple). Additional peaks between 0 and $-1 \mathrm{~V}$ are due to electroactive products resulting from the initial oxidation. 


\section{$\underline{\text { X-ray Crystallographic Details }}$}

The crystal data of 1a were collected on a BRUKER X8-APEX II diffractometer with a CCD area detector and multi-layer mirror monochromated $\mathrm{Mo}_{\mathrm{K} \alpha}$ radiation. The crystal data of $\mathbf{1 b}, \mathbf{2}, \mathbf{3 , 4}, \mathbf{7 ,} 8$ were acquired on a BRUKER D8 QUEST diffractometer with a CMOS area detector and multi-layer mirror monochromated $\mathrm{MoK}_{\alpha}$ radiation. The crystal data of $\mathbf{6 b}$ were collected on a RIGAKU SYNERGY DUALFLEX HYPIX diffractometer with a CMOS area detector and multi-layer mirror monochromated $\mathrm{Cu}_{\mathrm{K} \alpha}$ radiation. The structures were solved using the intrinsic phasing method, ${ }^{7}$ refined with the SHELXL program ${ }^{8}$ and expanded using Fourier techniques. All non-hydrogen atoms were refined anisotropically. Hydrogen atoms were included in structure factor calculations. Unless otherwise noted, all hydrogen atoms were assigned to idealized geometric positions.

Crystallographic data have been deposited with the Cambridge Crystallographic Data Center as supplementary publication nos. CCDC-1965307-1965313 and 1965520. These data can be obtained free of charge from The Cambridge Crystallographic Data Centre via www.ccdc.cam.ac.uk/data_request/cif

\section{$\underline{1 \mathbf{a}}$}

Crystal data for 1a: $\mathrm{C}_{26} \mathrm{H}_{31} \mathrm{~B}_{2} \mathrm{Br}_{2} \mathrm{~N}_{3}, M_{\mathrm{r}}=566.98$, orange plate, $0.141 \times 0.06 \times 0.043 \mathrm{~mm}^{3}$, triclinic space group $P \overline{1}, a=9.4709(6) \AA, b=10.8616(6) \AA, c=13.3353(10) \AA, \alpha=75.983(3)^{\circ}, \beta=70.506(2)^{\circ}$, $\gamma=86.232(2)^{\circ}, \quad V=1254.48(14) \AA^{3}, \quad Z=2, \quad \rho_{\text {calcd }}=1.501 \mathrm{~g} \cdot \mathrm{cm}^{-3}, \quad \mu=3.251 \mathrm{~mm}^{-1}, \quad F(000)=576$, $T=100(2) \mathrm{K}, R_{l}=0.0644, w R^{2}=0.0758,5327$ independent reflections $\left[2 \theta \leq 53.514^{\circ}\right]$ and 306 parameters.

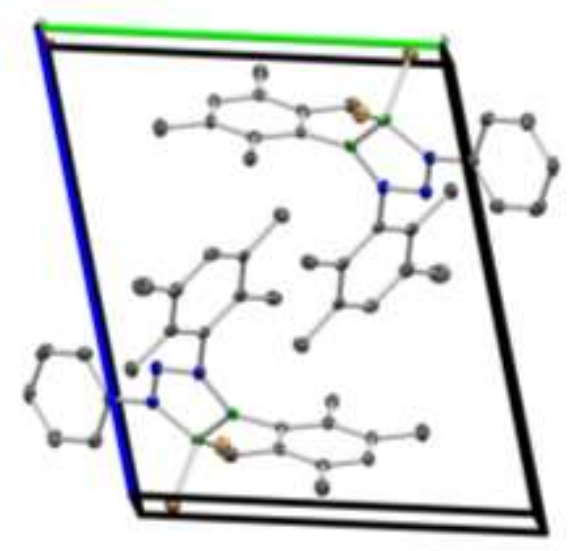

Figure S40: Packing in the solid-state structure of 1a seen from the a-axis perspective. Hydrogen atoms were removed for clarity. Orange $=$ bromine, green $=$ boron, blue $=$ nitrogen .

\section{$\underline{\mathbf{1 b}}$}

Very sensitive crystals form as polycrystalline material and recrystallizations resulted in decomposition. All crystals tended to be multi-domain and very poorly diffracting. Some signs of untreated multi-domain suggest that the sensible model obtained should be only used as a proof of connectivity. The cocrystallized hexane half-molecule was found to be disordered about a fourfold rotoinversion axis and modeled with part -1 (constrained to 0.5 occupancy). 
The distances between pairs of carbon atoms in the disordered hexane molecule were restrained during refinement to the same value $(1.54 \AA$ ) with SADI/DFIX restraints. The Uii displacement parameters of the carbon atoms in the disordered hexane molecule were restrained with the ISOR keyword to approximate isotropic behavior. The atomic displacement parameters of the carbon atoms in the disordered hexane molecule were restrained with the RIGU keyword in the ShelXL input ('enhanced rigid bond' restraint for all bonds in the connectivity list. Standard values of 0.004 for both parameters s1 and s2 were used).

Crystal data for $1 \mathbf{b}: \mathrm{C}_{27} \mathrm{H}_{34} \mathrm{~B}_{2} \mathrm{Cl}_{2} \mathrm{~N}_{3}, M_{\mathrm{r}}=493.09$, orange prism, $0.237 \times 0.222 \times 0.161 \mathrm{~mm}^{3}$, tetragonal space group $P \overline{4} 2_{1} c, a=16.7981(15) \AA, b=16.7981(15) \AA, c=19.5622(16) \AA, \alpha=90^{\circ}, \beta=90^{\circ}$, $\gamma=90^{\circ}, \quad V=5520.0(11) \AA^{3}, \quad Z=8, \quad \rho_{\text {calcd }}=1.187 \mathrm{~g} \cdot \mathrm{cm}^{-3}, \quad \mu=0.255 \mathrm{~mm}^{-1}, \quad F(000)=2088$, $T=100(2) \mathrm{K}, \quad R_{l}=0.1350, w R^{2}=0.1213,5361$ independent reflections $\left[2 \theta \leq 52.764^{\circ}\right]$ and 342 parameters.

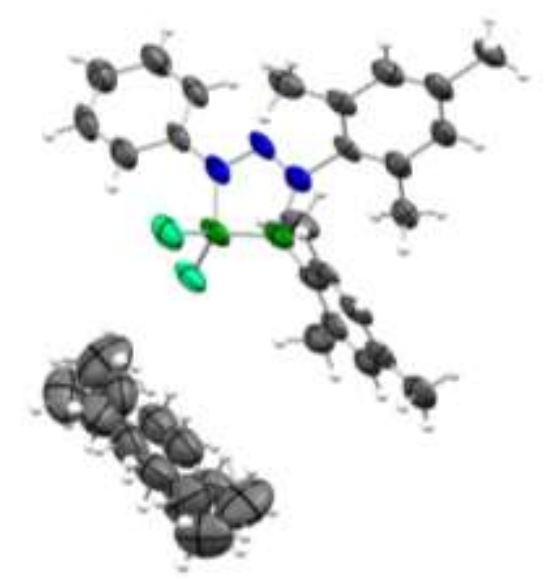

Figure S41: Solid-state structure of $\mathbf{1 b}$ showing the disordered hexane molecule in two different conformations generated by symmetry. Turquoise $=$ chlorine, green $=$ boron, blue $=$ nitrogen . 


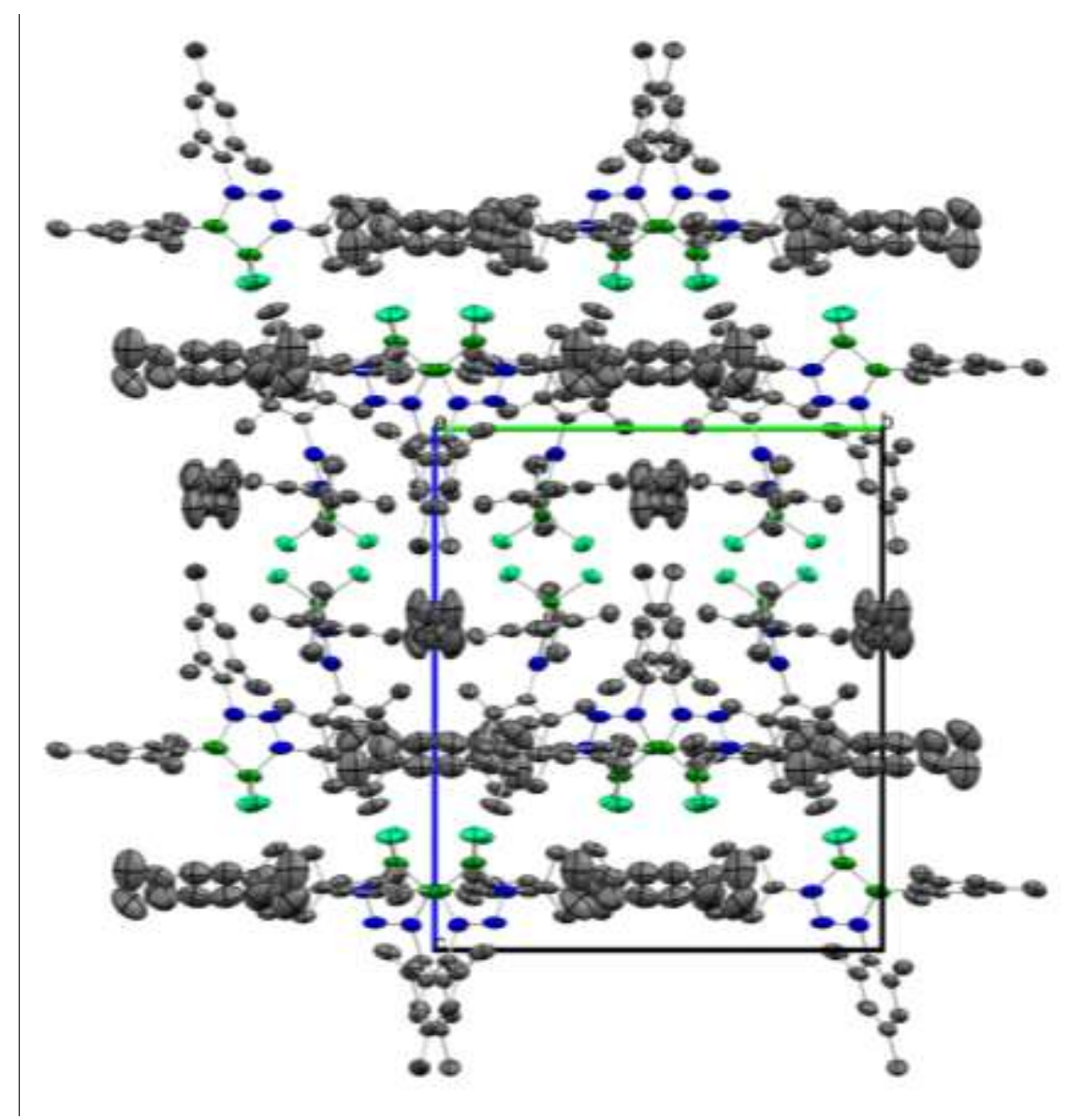

Figure S42: Packing in the solid-state structure of $\mathbf{1 b}$ seen from the b-axis perspective. Hydrogen atoms were removed for clarity. Turquoise $=$ chlorine, green $=$ boron, blue $=$ nitrogen

Crystal data for $2: \mathrm{C}_{46} \mathrm{H}_{34} \mathrm{~B}_{2} \mathrm{~F}_{2} \mathrm{~N}_{4}, M_{\mathrm{r}}=702.39$, orange block, $0.189 \times 0.134 \times 0.074 \mathrm{~mm}^{3}$, triclinic space group $P \overline{1}, a=10.182(3) \AA, \quad b=10.397(3) \AA, c=16.963(7) \AA, \alpha=81.522(14)^{\circ}, \beta=84.882(19)^{\circ}$, $\gamma=88.280(17)^{\circ}, \quad V=1768.9(10) \AA^{3}, \quad Z=2, \quad \rho_{\text {calcd }}=1.319 \mathrm{~g} \cdot \mathrm{cm}^{-3}, \quad \mu=0.084 \mathrm{~mm}^{-1}, \quad F(000)=732$, $T=100(2) \mathrm{K}, \quad R_{I}=0.0539, \quad w R^{2}=0.1183,7194$ independent reflections $\left[2 \theta \leq 52.74^{\circ}\right]$ and 487 parameters. 


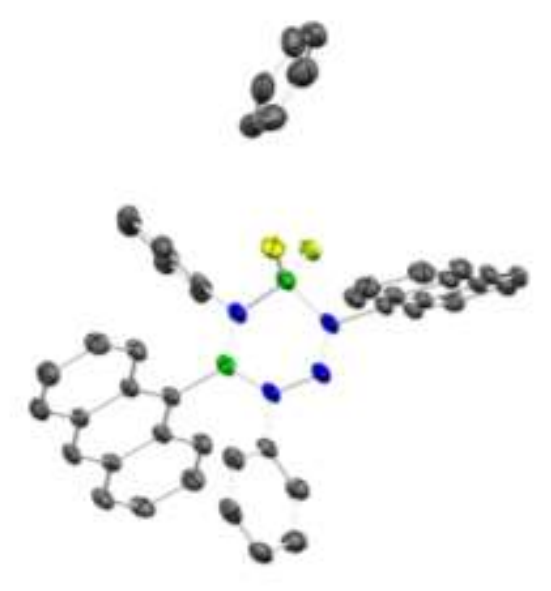

Figure S43: Solid-state structure of $\mathbf{2}$ showing the asymmetric unit, which contains one molecule of cocrystallized benzene. Hydrogen atoms were removed for clarity. Yellow-green $=$ fluorine, green $=$ boron, blue $=$ nitrogen .

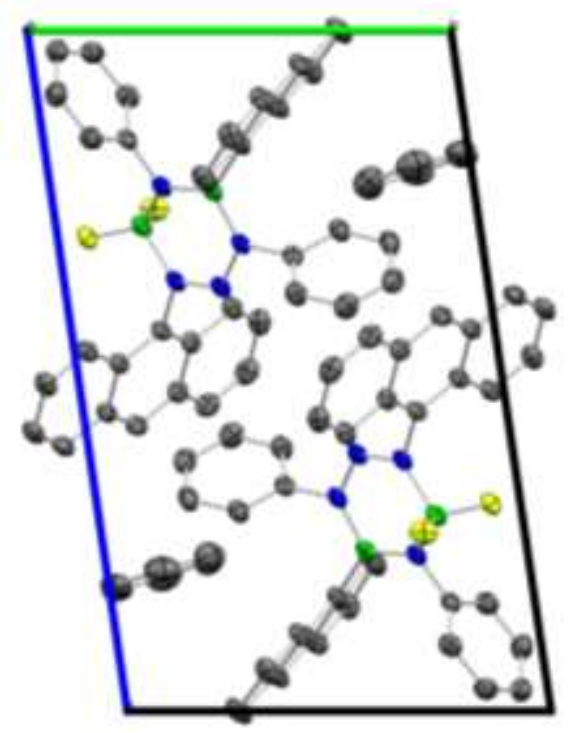

Figure S44: Packing in the solid-state structure of $\mathbf{2}$ seen from the a-axis perspective. Hydrogen atoms were removed for clarity. Yellow-green $=$ fluorine, green $=$ boron, blue $=$ nitrogen .

Reflections for this crystals were weak but nevertheless yielded a sensible model that strongly supports the proposed connectivity.

The cocrystallized benzene molecule was found to be in two positions related by a rotation along an axis going through the plane of the molecule. This was refined as a two-part disorder with a refined ratio of 0.76:0.24. The displacement parameters of atoms $\mathrm{C} 1>\mathrm{C} 6 \mathrm{a}$ of the disordered benzene moiety were restrained to the same value with similarity restraint SIMU. The Uii displacement parameters of $\mathrm{C} 1>\mathrm{C} 6 \mathrm{a}$ of the disordered benzene moiety were restrained with the ISOR keyword to approximate isotropic behavior. The atomic displacement parameters of atoms $\mathrm{C} 1>\mathrm{C} 6$ and $\mathrm{C} 1 \mathrm{a}>\mathrm{C} 6 \mathrm{a}$ of the disordered benzene moiety were restrained with the RIGU keyword in the ShelXL input ('enhanced rigid bond' restraint for all bonds in the connectivity list. Standard values of 0.004 for both parameters 
$\mathrm{s} 1$ and s2 were used). The 1-2 and 1-3 distances in $\mathrm{C} 1>\mathrm{C} 6$ of the disordered benzene moiety were restrained to the same values with SAME.

Crystal data for 3: $\mathrm{C}_{40} \mathrm{H}_{29} \mathrm{~B}_{2} \mathrm{Br}_{2} \mathrm{~N}_{3}, M_{\mathrm{r}}=733.10$, red needle, $0.158 \times 0.041 \times 0.036 \mathrm{~mm}^{3}$, monoclinic space group $P 2_{1} / c, \quad a=9.068(3) \AA, \quad b=23.484(5) \AA, \quad c=15.519(5) \AA, \quad \beta=97.70(3)^{\circ}$, $V=3274.8(16) \AA^{3}, \quad Z=4, \quad \rho_{\text {calcd }}=1.487 \mathrm{~g} \cdot \mathrm{cm}^{-3}, \quad \mu=2.510 \mathrm{~mm}^{-1}, \quad F(000)=1480, \quad T=100 \mathrm{~K}$, $R_{l}=0.1488, w R^{2}=0.1240,6153$ independent reflections $\left[2 \theta \leq 51.356^{\circ}\right]$ and 479 parameters.

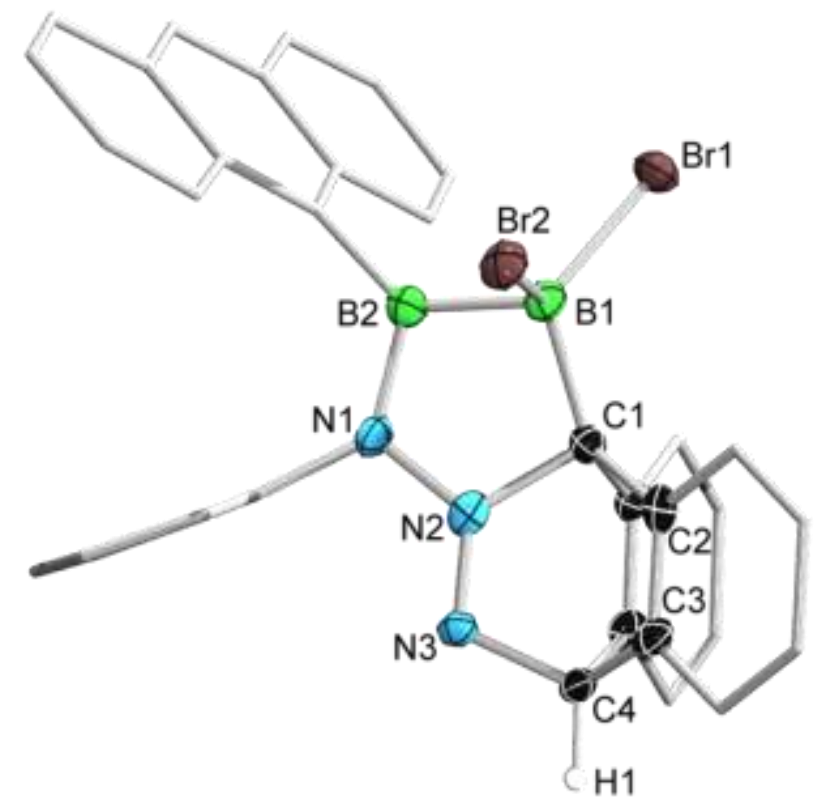

Figure S45: Structural parameters of 3. Selected bond distances (Å): N1-N2 1.389(6), N2-N3 1.254(7), N3-C4 1.515(7), N2-C1 1.563(7), C4-C3 1.506(7), C1-C2 1.530(7), N1-B2 1.456(9), B2-B1 1.66(1), $\mathrm{B} 1-\mathrm{C} 1$ 1.625(8), B1-Br1 1.987(8), B1-Br2 2.122(7). Red-brown = bromine, green = boron, blue = nitrogen. 

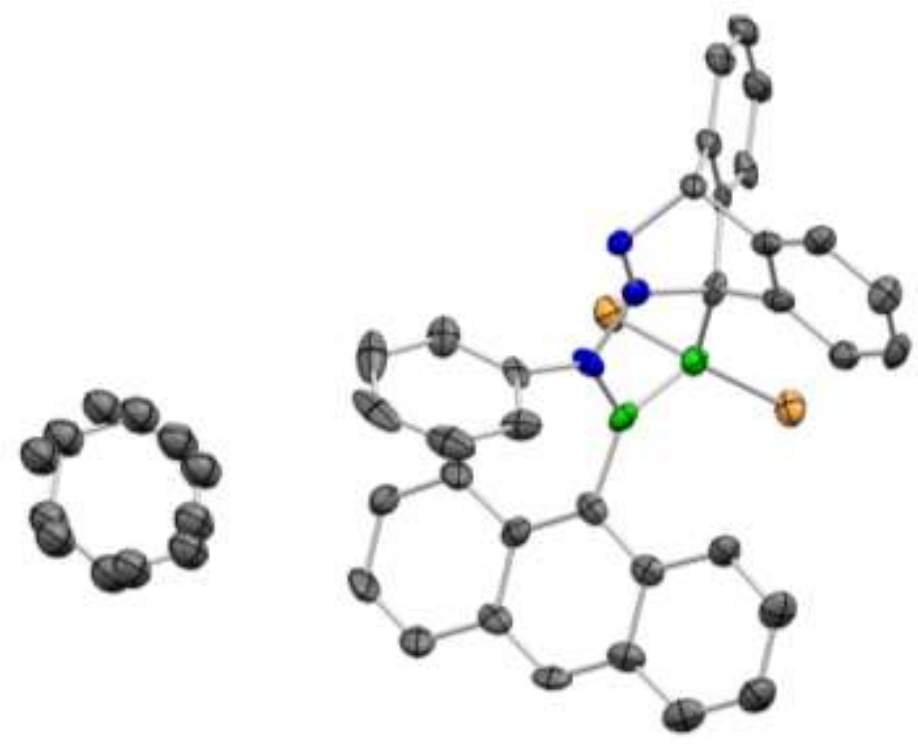

Figure S46: Solid-state structure of $\mathbf{3}$ showing the asymmetric unit and its disordered cocrystallized benzene molecule. Hydrogen atoms are omitted for clarity. Orange $=$ bromine, green $=$ boron, blue $=$ nitrogen.

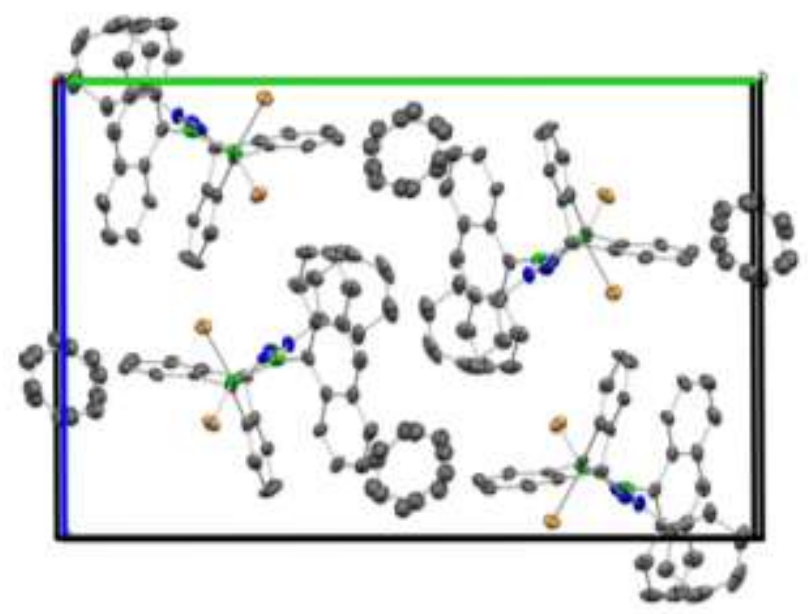

Figure S47: Packing in the solid-state structure of $\mathbf{3}$ from the b-axis perspective. Hydrogen atoms are omitted for clarity. Orange $=$ bromine, green $=$ boron, blue $=$ nitrogen .

\section{$\underline{4}$}

All hydrogen atoms except H1_4 and H1_5 were assigned to idealized positions. The coordinates of H1_4 and H1_5 were refined freely but restrained to a N-H distance of $0.88 \AA$ with a DFIX command.

Crystal data for 4: $\mathrm{C}_{34} \mathrm{H}_{26} \mathrm{~B}_{2} \mathrm{Br}_{6} \mathrm{~N}_{2}, M_{\mathrm{r}}=963.65$, orange block, $0.359 \times 0.322 \times 0.210 \mathrm{~mm}^{3}$, monoclinic space group $P 2_{1} / c, \quad a=13.475(3) \AA, \quad b=19.327(3) \AA, \quad c=14.243(3) \AA, \quad \beta=114.592(16)^{\circ}$, 
$V=3372.8(12) \AA^{3}, \quad Z=4, \quad \rho_{\text {calcd }}=1.898 \mathrm{~g} \cdot \mathrm{cm}^{-3}, \quad \mu=7.170 \mathrm{~mm}^{-1}, \quad F(000)=1856, \quad T=100(2) \quad \mathrm{K}$, $R_{l}=0.0333, w R^{2}=0.0699,6639$ independent reflections $\left[2 \theta \leq 52.04^{\circ}\right]$ and 405 parameters.

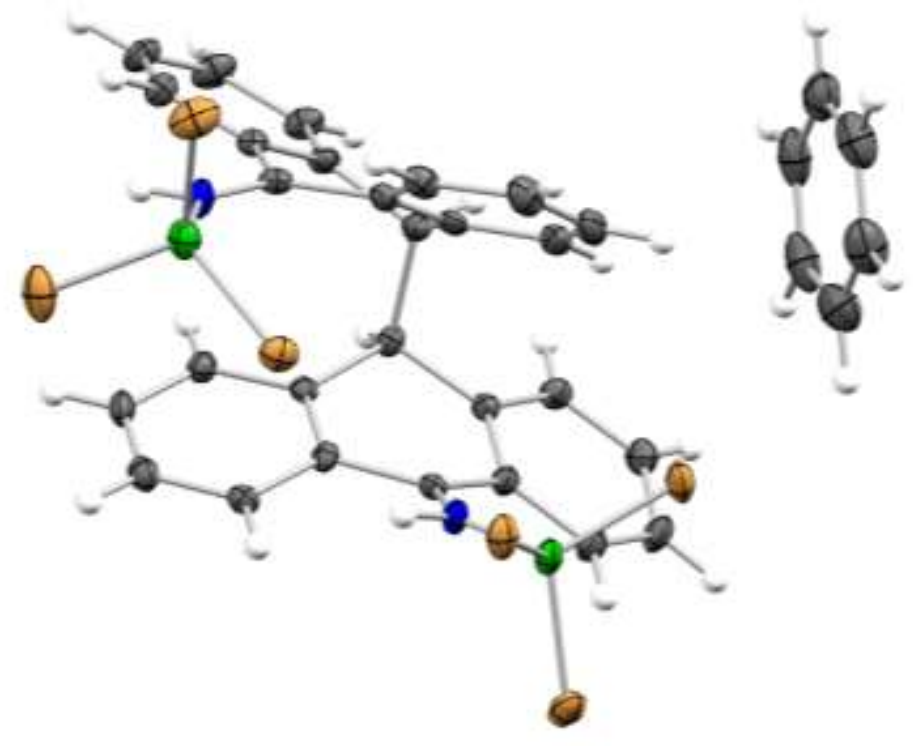

Figure S48: Solid-state structure of $\mathbf{4}$ showing the asymmetric unit and its cocrystallized benzene molecule. Hydrogen atoms are omitted for clarity. Orange $=$ bromine, green $=$ boron, blue $=$ nitrogen .

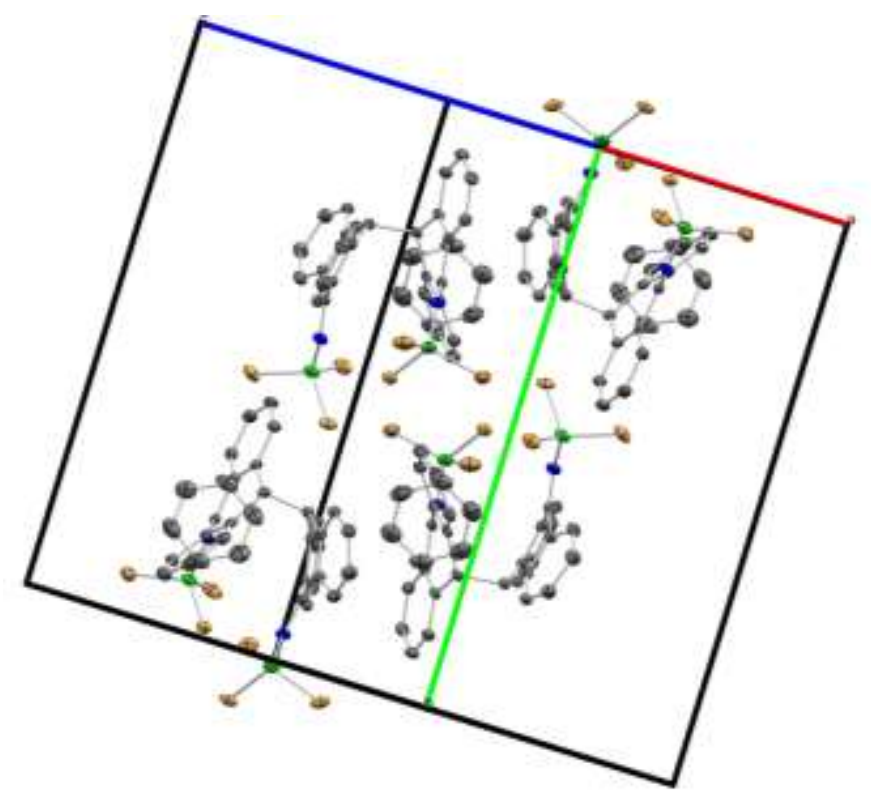

Figure S49: Packing in the solid-state structure of 4 . Hydrogen atoms are omitted for clarity. Orange = bromine, green $=$ boron, blue $=$ nitrogen . 
The azide functional group was found in two orientations approximately related by a rotation of the groups about the B-N bond. This was refined as a two-part disorder with a refined ratio of 0.61:0.39. The displacement parameters of atoms N5 > N7a of the disordered azide moiety were restrained to the same value with similarity restraint SIMU. The Uii displacement parameters of N5 > N7a of the disordered azide moiety were restrained with the ISOR keyword to approximate isotropic behavior. The atomic displacement parameters of atoms N5 > N7 and N5a > N7a of the the disordered azide moiety were restrained with the RIGU keyword in the ShelXL input ('enhanced rigid bond' restraint for all bonds in the connectivity list; standard values of 0.004 for both parameters s1 and s2 were used). The 1-2 and 1-3 distances for N5 > N7 and N5a> N7a of the disordered azide moiety were restrained to the same values with SAME.

Crystal data for 6b: $\mathrm{C}_{23} \mathrm{H}_{35} \mathrm{~B}_{2} \mathrm{~N}_{7} \mathrm{Si} . M_{\mathrm{r}}=459.29$, colorless block, $0.141 \mathrm{x} 0.111$ x $0.069 \mathrm{~mm}^{3}$, monoclinic space group $P 22_{1} / n, a=11.2213$ (2) $\AA, b=14.5864(2) \AA, c 16.2503$ (3) $\AA, \beta=103.500(2)^{\circ}$, $V=2586.33(3) \AA^{3}, Z=4, \rho_{\text {calcd }}=1.18 \mathrm{~g} \cdot \mathrm{cm}^{-3}, \mu=0.983 \mathrm{~mm}^{-1}, F(000)=984, T=100 \mathrm{~K}, R_{l}=0.0456$, $w R^{2}=0.1087,4920$ independent reflections $\left[2 \theta \leq 35.0345^{\circ}\right]$ and 337 parameters.

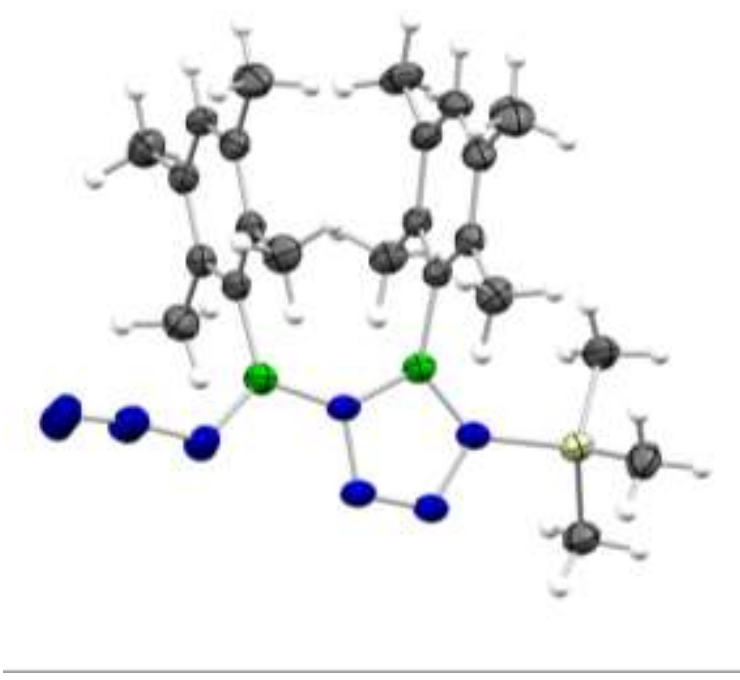

Figure S50: Solid-state structure of $\mathbf{6 b}$, showing the asymmetric unit and the disordered azide moiety. Beige $=$ silicon, green $=$ boron, blue $=$ nitrogen . 


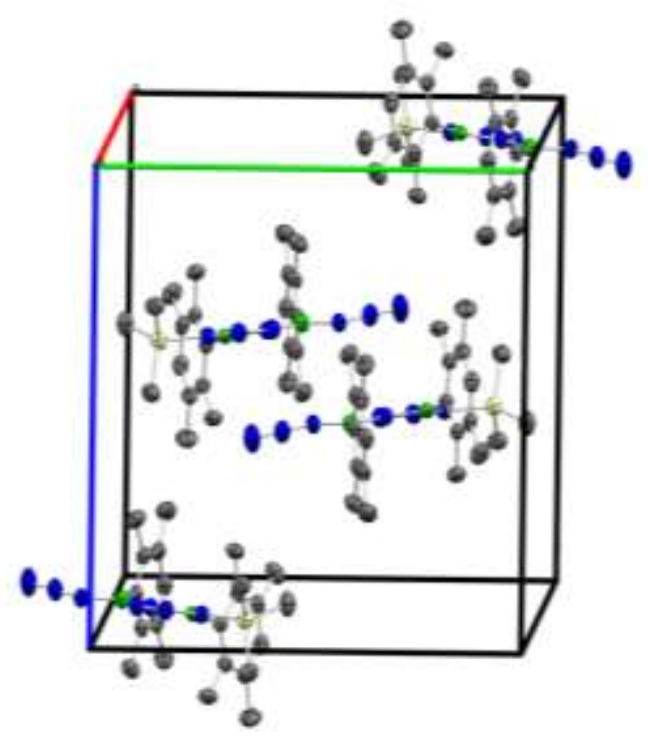

Figure S51: Solid-state structure of $\mathbf{6 b}$, showing the packing from a-axis perspective. Hydrogen atoms were omited for clarity. Beige $=$ silicon, green $=$ boron, blue $=$ nitrogen .

\section{$\underline{7}$}

The structure was refined as an inversion twin using the TWIN keyword with a refined BASF parameter of 0.37861 . The two B-N 3 sites were found to be substitutionally disordered with bromine atoms and were refined as two-part disorders with refined azide-to-bromide ratios of 0.98:0.02 and 0.96:0.04, respectively. The displacement parameters of atoms $\mathrm{N} 1$ and $\mathrm{Br} 2$ of one of the azide/bromide disordered sites were constrained to the same value with the EADP keyword. The displacement parameters of atoms $\mathrm{N} 4>\mathrm{Br} 1$ of one of the Br/azide substitutional disorders were restrained to the same value with similarity restraint SIMU.

Crystal data for 7: diazadib $\mathrm{C}_{36} \mathrm{H}_{44} \mathrm{~B}_{4} \mathrm{Br}_{0.06} \mathrm{~N}_{7.82}, M_{\mathrm{r}}=634.23$, colorless prism (rhombus), $0.349 \times 0.244 \times 0.20 \mathrm{~mm}^{3}$, orthorhombic space group Pna2 $1, a=22.0572(7) \AA, \quad b=7.7994(2) \AA$, $c=20.9401(6) \AA, V=3602.39(18) \AA^{3}, Z=4, \rho_{\text {calcd }}=1.169 \mathrm{~g} \cdot \mathrm{cm}^{-3}, \mu=0.134 \mathrm{~mm}^{-1}, F(000)=1347$, $T=105(2) \mathrm{K}, R_{I}=0.0413, w R^{2}=0.0964,7630$ independent reflections $\left[2 \theta \leq 53.496^{\circ}\right]$ and 460 parameters 


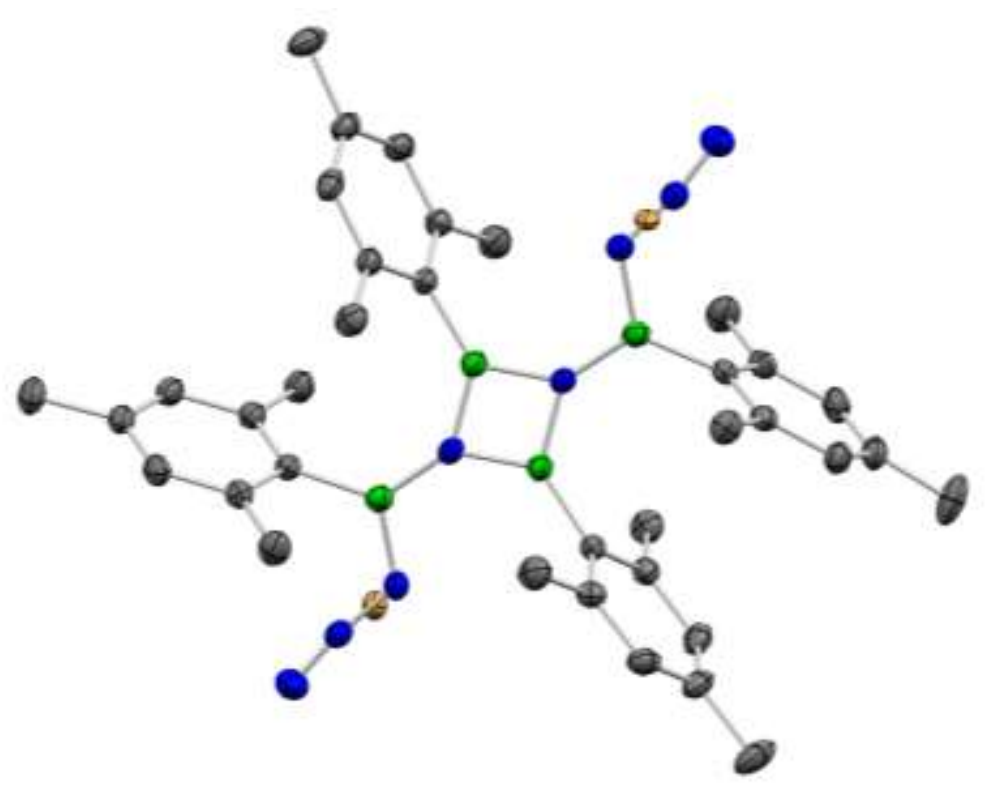

Figure S52: Solid-state structure of 7 showing the asymmetric unit with the azide/bromide substitutional disorder. Hydrogen atoms were omitted for clarity. Orange $=$ bromine, green $=$ boron, blue $=$ nitrogen .

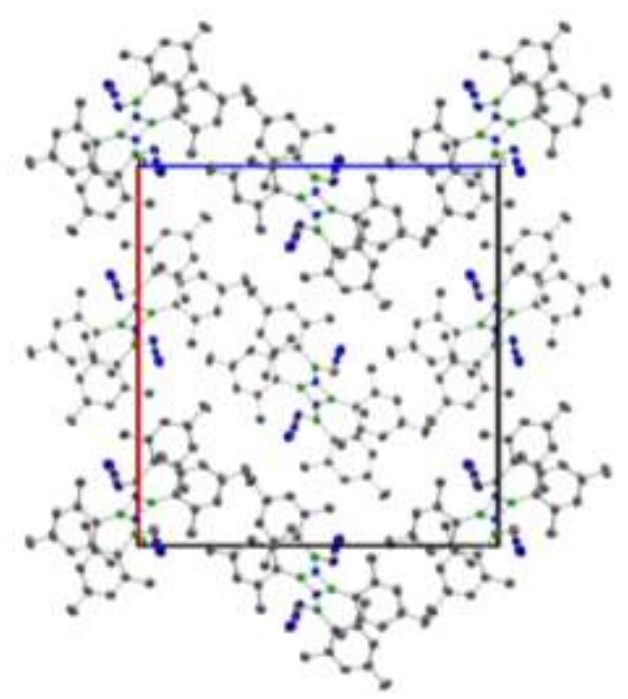

Figure S53: Packing in the solid-state structure of 7 from the b-axis perspective. The bromide/azide substitutional disorder is also shown. Hydrogen atoms were omitted for clarity. Orange = bromine, green $=$ boron, blue $=$ nitrogen . 


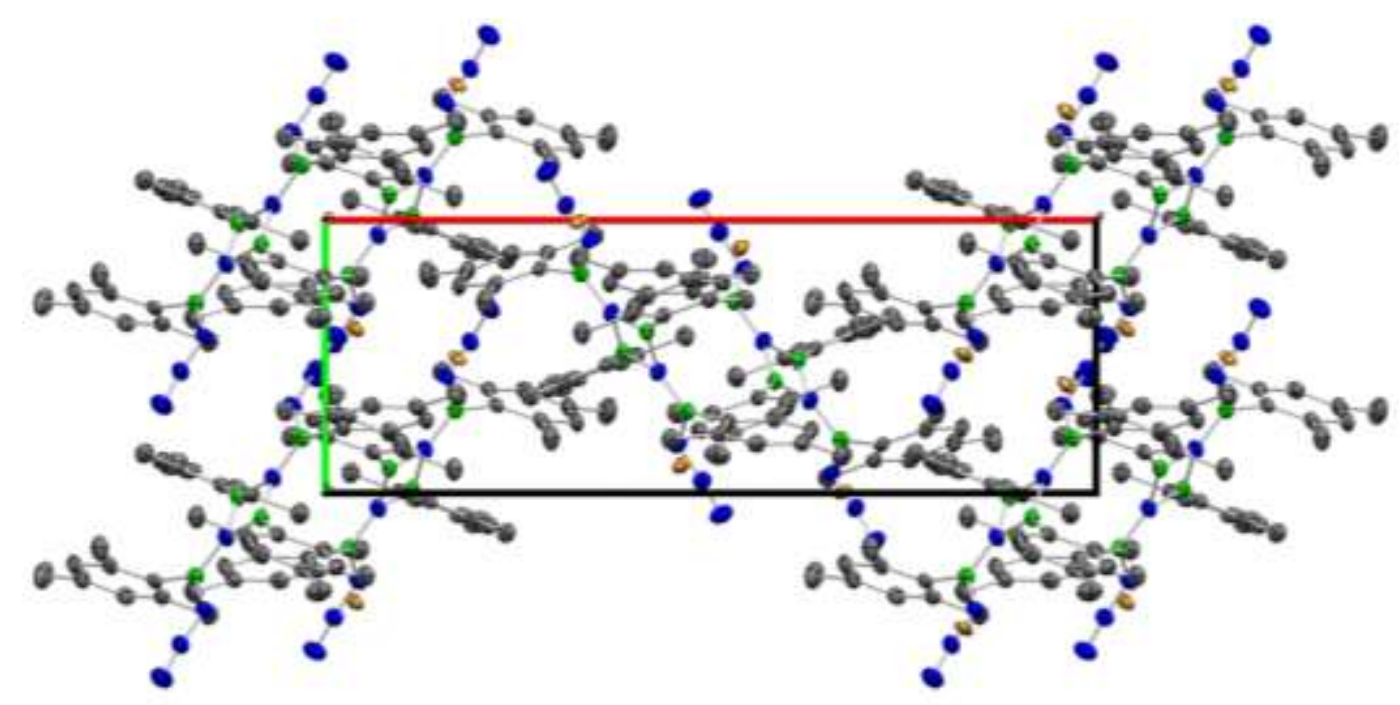

Figure S54: Packing in the solid-state structure of 7 from the c-axis perspective. The bromide/azide substitutional disorder is also shown. Hydrogen atoms were omitted for clarity. Orange = bromine, green $=$ boron, blue $=$ nitrogen .

\section{8}

The crystal was a pseudo-merohedral twin with domains rotated by $180.0^{\circ}$ around real axis $[1.000$ $0.0420 .066]$. The structure was refined using the TWIN keyword with a BASF parameter refined to 0.41 .

The compound crystallizes as small, twinned and relatively weakly diffracting crystals. The model presented a problematic chlorine ellipsoid, which indicated an (expected) substitutional disorder. With a restrained azide moiety (B2-N5 distance restrained to $1.6 \AA$, N5-N6 distance restrained to $1.22 \AA$, N6-N7 distance restrained to $1.1 \AA$ with DFIX commands), this could be modeled in a sensible manner. The refined ratio of the chloride:azide disorder was 0.77:0.23. The displacement parameters of atoms Cl1, N5, N6, N7, of the azide/chloride substitutionally disordered site were restrained to the same value with similarity restraint SIMU. The Uii displacement parameters of atoms N5, N6, N7 of the azide/chloride substitutional disordered site were restrained with the ISOR keyword to approximate isotropic behavior. The atomic displacement parameters of atoms N5, N6, N7 of the azide/chloride substitutional disordered site were restrained with RIGU keyword in ShelXL input ('enhanced rigid bond' restraint for all bonds in the connectivity list; standard values of 0.004 for both parameters s1 and s2 were used).

Crystal data for 8: $\mathrm{C}_{42} \mathrm{H}_{62} \mathrm{~B}_{4} \mathrm{Cl}_{1.53} \mathrm{~N}_{9.41} \mathrm{Si}_{2}, \quad M_{\mathrm{r}}=852.41$, colorless block, $0.83 \times 0.277 \times 0.24 \mathrm{~mm}^{3}$, triclinic space group $P \overline{1}, a=10.3817(6) \AA, b=11.2612(6) \AA, c=11.3805(5) \AA, \alpha=116.312(2)^{\circ}$, $\beta=92.980(2)^{\circ}, \quad \gamma=90.859(2)^{\circ}, \quad V=1189.96(11) \AA^{3}, \quad Z=1, \quad \rho_{\text {calcd }}=1.190 \mathrm{~g} \cdot \mathrm{cm}^{-3}, \quad \mu=0.201 \mathrm{~mm}^{-1}$, $F(000)=454, T=100(2) \mathrm{K}, R_{l}=0.0557, w R^{2}=0.1274,4477$ independent reflections $\left[2 \theta \leq 51.348^{\circ}\right]$ and 300 parameters. 


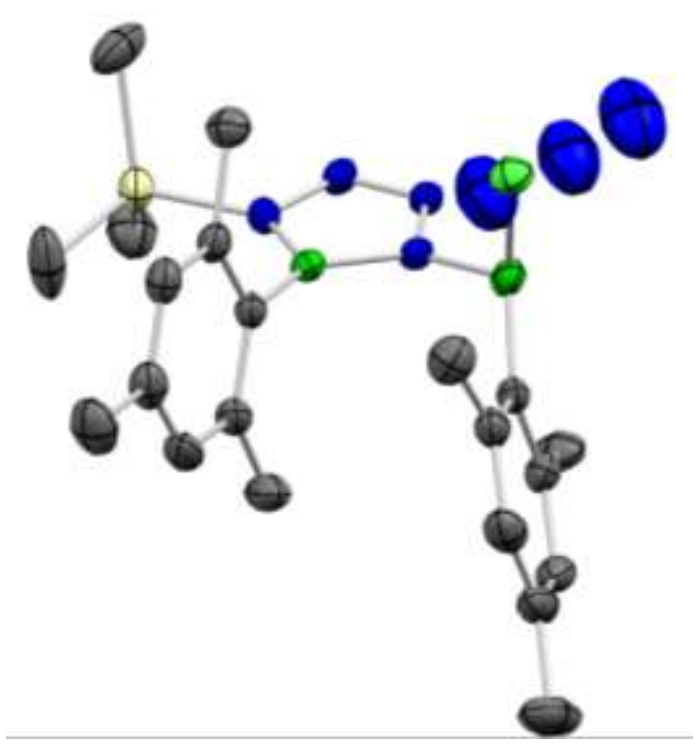

Figure S55: Solid-state structure of $\mathbf{8}$ showing the asymmetric unit. The substitutional chloride/azide disorder is shown here with the two superimposed moieties. Hydrogen atoms are omitted for clarity. Beige $=$ silicon, green $=$ boron, blue $=$ nitrogen .

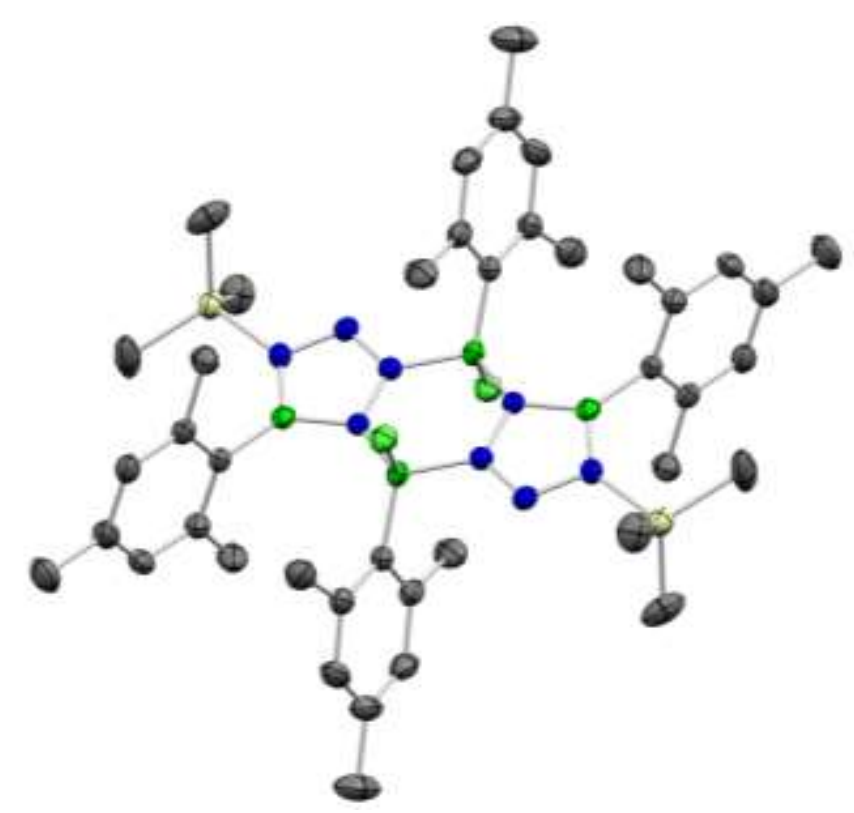

Figure S56: Solid-state structure of $\mathbf{8}$ showing the whole boryl-tetrazaborole dimer molecule generated by symmetry. The subtitutionally-disordered azide moiety (see Figure S55) is omitted. Hydrogen atoms are omitted for clarity. Beige $=$ silicon, pale green $=$ chlorine, green $=$ boron, blue $=$ nitrogen . 


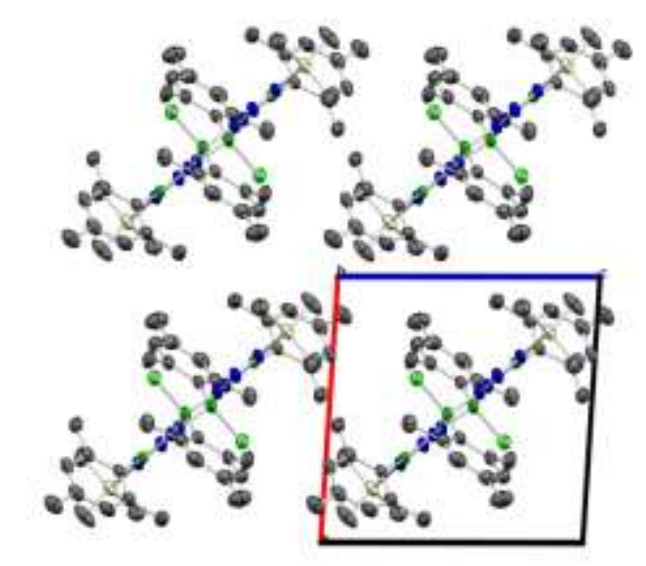

Figure S57: Packing in the solid-state structure of $\mathbf{8}$ from the b-axis perspective. The substitutionally disordered azide moiety (see Figure S55) is omitted. Hydrogen atoms are omitted for clarity. Beige = silicon, pale green $=$ chlorine, green $=$ boron, blue $=$ nitrogen .

\section{Computational Methodology}

DFT calculations were carried out at the M06-2X level of theory, ${ }^{9}$ which has been found to be good for studying main-group thermochemistry and kinetics. All structures/species studied here were fully optimized in benzene solvent employing the SMD model. ${ }^{10}$ The $6-31 \mathrm{G}^{* *}$ basis set was used to describe C, H, B, N, F, Si and Cl. The effective core potentials (ECPs) of Hay and Wadt with double- $\zeta$ valence basis sets (LanL2DZ) ${ }^{11-12}$ were chosen to describe the $\mathrm{Br}$ atom. In addition, polarization functions were added for $\operatorname{Br}\left(\zeta_{d}=0.428\right) .{ }^{13}$ Frequency calculations were performed to ensure that a transition state has only one imaginary frequency and a local minimum has no imaginary frequencies. Intrinsic reaction coordinate (IRC) ${ }^{14-15}$ calculations were run to ensure that transition states connect relevant minima. ${ }^{11} \mathrm{~B}$ NMR chemical shifts were calculated using the GIAO method, using $\mathrm{BF}_{4}{ }^{-}$as a reference $\left(\delta\left({ }^{11} \mathrm{~B}\right)=-1.4 \mathrm{ppm}\right)$. All the calculations were performed using the Gaussian 09 package. ${ }^{16}$ 


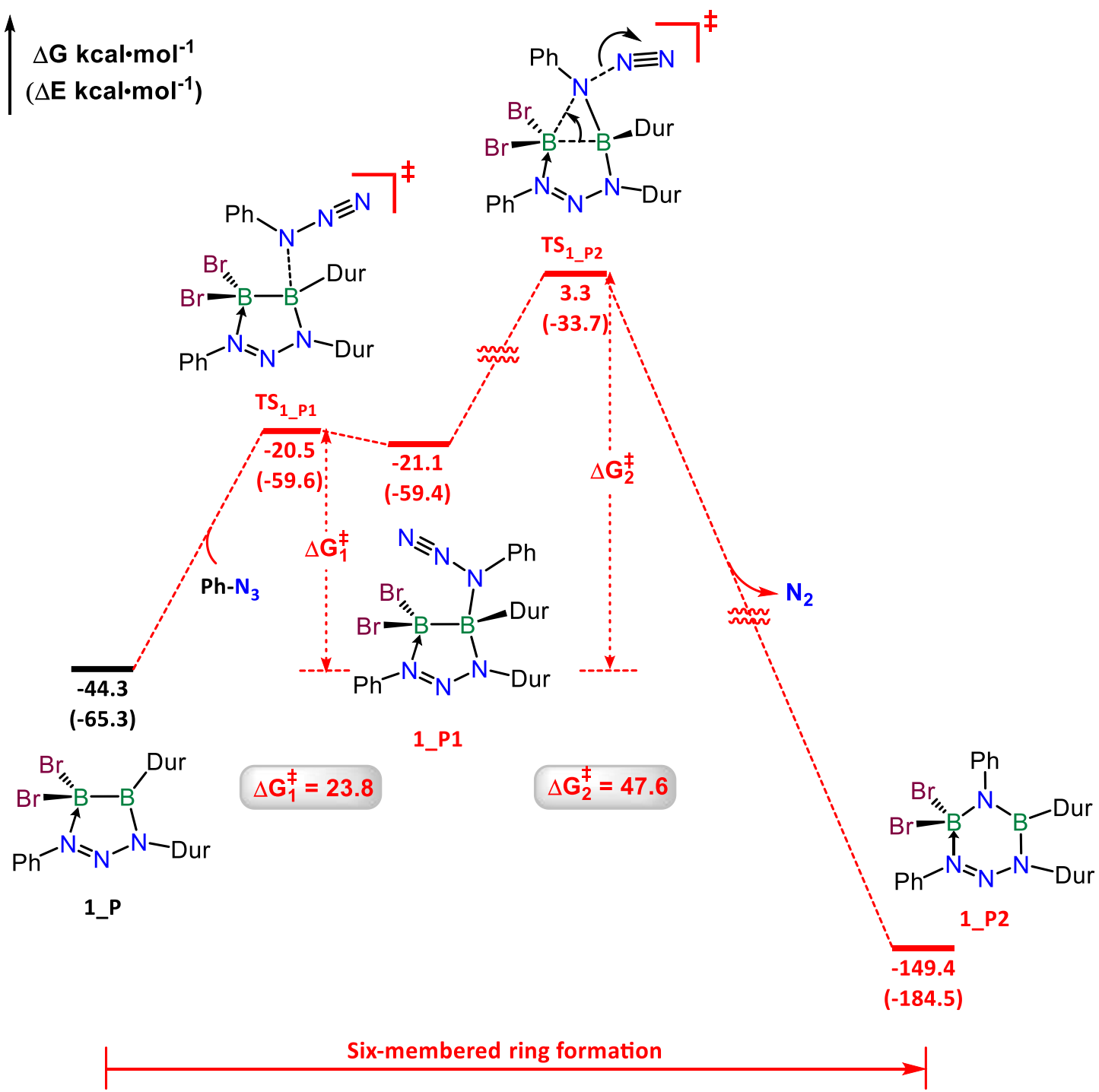

Scheme S1: Gibbs free energy profile calculated for the putative reaction of $\mathbf{1} \_\mathbf{P}$ with $\mathrm{PhN}_{3}$ leading to the six-membered ring product 1_P2. The relative free energies and electronic energies (in parentheses) are given in $\mathrm{kcal} \cdot \mathrm{mol}^{-1}$. 

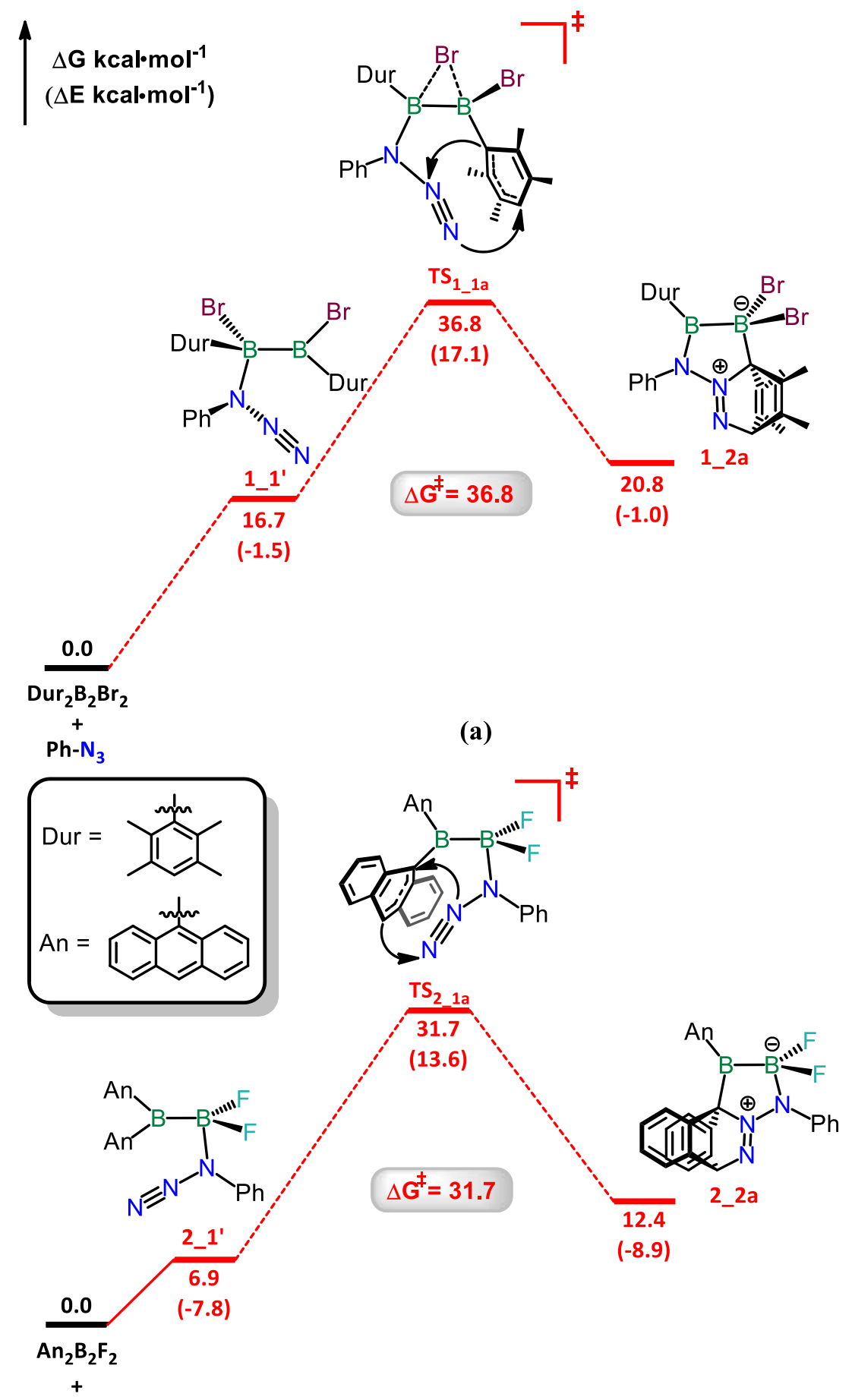

$\mathrm{Ph}-\mathrm{N}_{3}$

(b)

[4+2] Cycloaddition

Scheme S2: Gibbs free energy profile calculated for the various possible reaction pathways in the reaction of (a) Dur $\mathbf{B}_{2} \mathbf{B r}_{2}$ with phenyl azide leading to the [4+2] cycloaddition product 1_2a, and (b) $\mathbf{A n}_{2} \mathbf{B}_{2} \mathbf{F}_{2}$ with phenyl azide leading to the [4+2] cycloaddition product 2_2a. The relative free energies and electronic energies (in parentheses) are given in $\mathrm{kcal} \cdot \mathrm{mol}^{-1}$ 


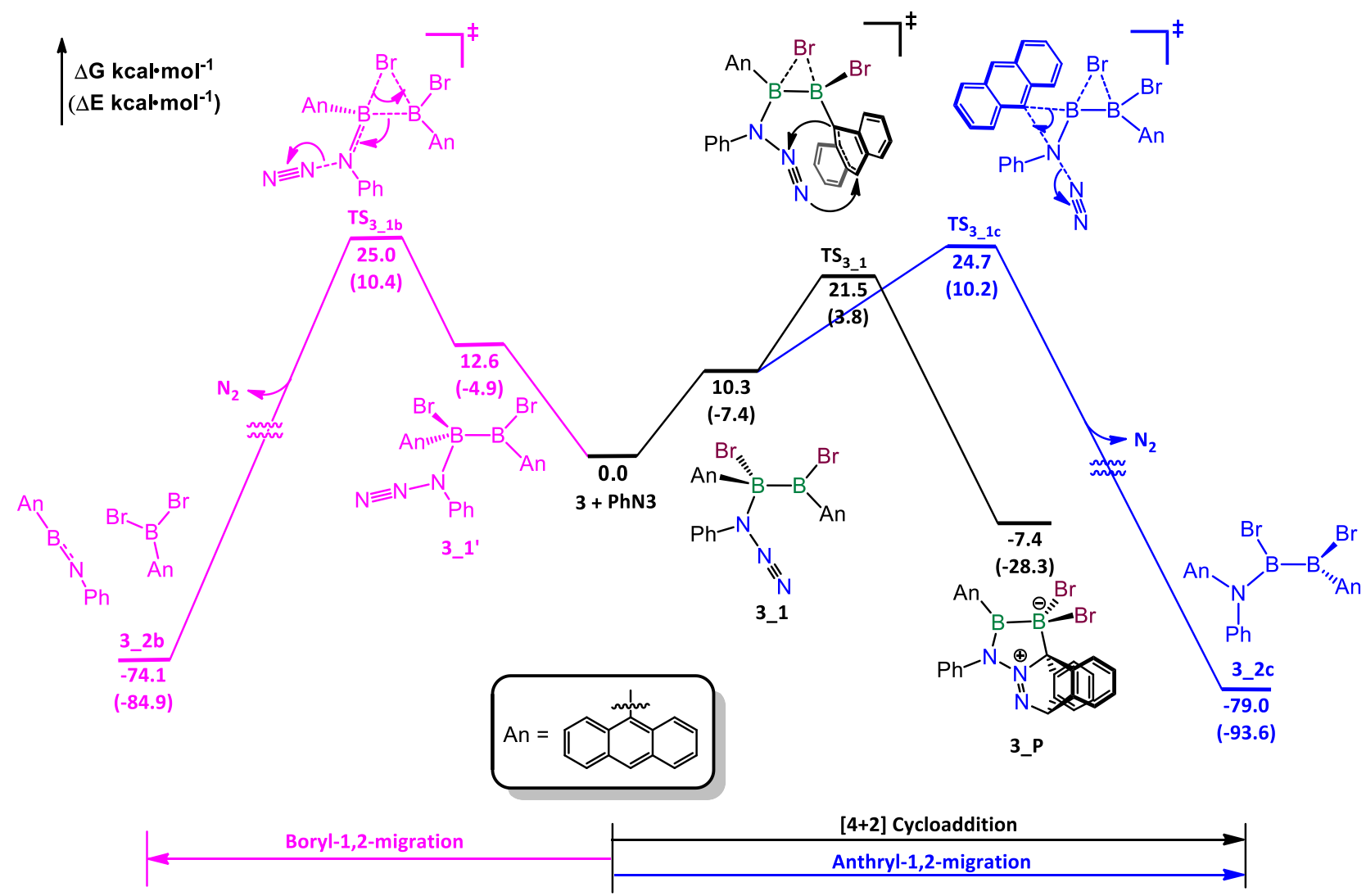

Scheme S3: Gibbs free energy profile calculated for the various possible reaction pathways in the reaction of $\mathbf{A n}_{2} \mathbf{B}_{2} \mathbf{B r} \mathbf{r}_{2}$ with $\mathrm{PhN}_{3}$. The relative free energies and electronic energies (in parentheses) are given in $\mathrm{kcal} \cdot \mathrm{mol}^{-1}$.

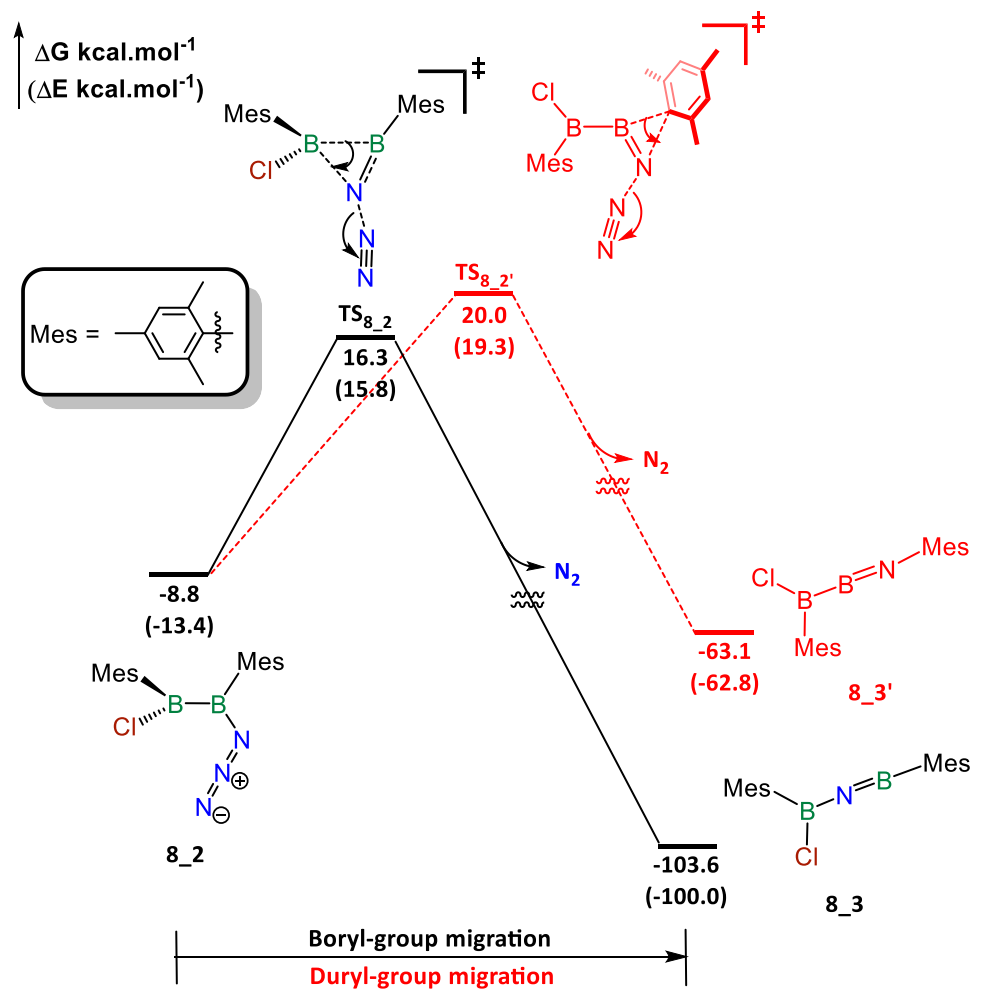


Scheme S4: Comparison of two possible pathways resulting from the formation of diborane azide 8_2. The path in black involves the insertion of the nitrene-like nitrogen of $\mathbf{T S}_{\mathbf{8}_{-} 2}$ into the boron-boron bond and leads to the final product 5a (see Main Article) while the alternative path in red involves the insertion of the nitrene-like nitrogen atom into the boron-mesityl bond and would lead to B-boryl iminoborane 8_3'.

\section{Calculated Cartesian Coordinates}

\begin{tabular}{|c|c|c|c|}
\hline \multicolumn{4}{|l|}{14} \\
\hline \multicolumn{4}{|c|}{$\mathrm{PhN3}$} \\
\hline 7 & -1.46836800 & -0.91631300 & 0.00004600 \\
\hline 7 & -2.39303200 & -0.09852700 & -0.00005500 \\
\hline 7 & -3.30788600 & 0.56264500 & -0.00005700 \\
\hline 6 & -0.15179100 & -0.37720200 & 0.00010900 \\
\hline 6 & 0.88606000 & -1.30920000 & 0.00001700 \\
\hline 6 & 0.12586900 & 0.99143400 & 0.00006200 \\
\hline 6 & 2. 20331300 & -0.86894500 & -0.00005800 \\
\hline 6 & 1. 44984500 & 1. 41799700 & 0.00000800 \\
\hline 6 & 2. 49205100 & 0.49479500 & -0.00003300 \\
\hline 1 & 0.64079900 & -2.36603800 & -0.00002200 \\
\hline 1 & -0.68310100 & 1. 71737300 & 0.00004500 \\
\hline 1 & 3. 00864200 & -1.59666900 & -0.00011700 \\
\hline 1 & 1. 66463900 & 2. 48194700 & -0.00001800 \\
\hline 1 & 3.52194400 & 0.83548100 & -0.00005600 \\
\hline \multicolumn{4}{|l|}{16} \\
\hline \multicolumn{4}{|c|}{ TMSN3 } \\
\hline 14 & 0.66188800 & -0.00222800 & 0.02827100 \\
\hline 7 & -0.82389000 & 0.09044900 & -0.96301700 \\
\hline 7 & -1.91842900 & 0.03682100 & -0.42208800 \\
\hline 7 & -2.96274800 & -0.00575700 & 0.01285900 \\
\hline 6 & 0.66876200 & -1.65976200 & 0.89484900 \\
\hline 6 & 2. 04749900 & 0.17772700 & -1.20268200 \\
\hline 6 & 0.59920600 & 1. 39661500 & 1. 26804200 \\
\hline 1 & 0.67313100 & -2.48343000 & 0.17467300 \\
\hline 1 & 1. 55118500 & -1.76542600 & 1. 53472800 \\
\hline 1 & -0.21619800 & -1.77437400 & 1. 53034800 \\
\hline 1 & 1. 98723700 & 1. 13930700 & -1.72101400 \\
\hline 1 & 3. 02236700 & 0.11955300 & -0.70844200 \\
\hline 1 & 2. 00462500 & -0.61420100 & -1.95649700 \\
\hline 1 & 0.56643000 & 2. 36842800 & 0.76639200 \\
\hline 1 & -0.28874300 & 1. 31843300 & 1. 90479300 \\
\hline 1 & 1. 47619800 & 1. 38483200 & 1. 92368300 \\
\hline \multicolumn{4}{|l|}{50} \\
\hline \multicolumn{4}{|c|}{ Dur2B2Br2 } \\
\hline 6 & 1. 85786900 & 0.13699300 & 0.37239900 \\
\hline 6 & 1. 73620800 & 1. 40203700 & 0.97592000 \\
\hline 6 & 2. 76391600 & 2. 34259200 & 0.81874900 \\
\hline 6 & 3. 88118800 & 1. 99807500 & 0.05903100 \\
\hline 1 & 4. 67652100 & 2. 73030700 & -0.06495800 \\
\hline 6 & 4. 01561400 & 0.75056700 & -0.54762900 \\
\hline 6 & 2. 99209200 & -0.19600100 & -0.38730300 \\
\hline 6 & -1.85839600 & 0.13617500 & -0.37206500 \\
\hline 6 & -2.99209200 & -0.19855600 & 0.38770700 \\
\hline 6 & -4.01679200 & 0.74660300 & 0.54851800 \\
\hline 6 & -3.88390300 & 1. 99462800 & -0.05748600 \\
\hline 1 & -4.68009300 & 2. 72583200 & 0.06702600 \\
\hline 6 & -2.76713600 & 2. 34089300 & -0.81712500 \\
\hline 6 & -1.73834800 & 1. 40161000 & -0.97498900 \\
\hline 5 & 0.67127000 & -0.86732400 & 0.50609800 \\
\hline 5 & -0.67072000 & -0.86680000 & -0.50586100 \\
\hline 35 & 0.66113500 & -2.17522800 & 1.96241100 \\
\hline 35 & -0.65774400 & -2.17235800 & -1.96447700 \\
\hline 6 & 3. 11950900 & -1.54640100 & -1.04860100 \\
\hline 1 & 3. 15147700 & -1.45466600 & -2.14040100 \\
\hline 1 & 4. 03956800 & -2.05536000 & -0.74113500 \\
\hline
\end{tabular}

\begin{tabular}{|c|c|c|c|}
\hline 1 & 2. 28712800 & -2.21342500 & -0.80588000 \\
\hline 6 & 5. 24240000 & 0.42467400 & -1.35799400 \\
\hline 1 & 5. 79177300 & -0.42164600 & -0.93086200 \\
\hline 1 & 4. 98450800 & 0.15025900 & -2.38672600 \\
\hline 1 & 5. 92235400 & 1. 27878600 & -1.39785700 \\
\hline 6 & 2. 66532900 & 3. 70135300 & 1. 46060600 \\
\hline 1 & 1. 78900100 & 4. 25355400 & 1. 10243000 \\
\hline 1 & 2.56910600 & 3. 62515600 & 2. 54941500 \\
\hline 1 & 3.55155000 & 4. 30166800 & 1. 24256700 \\
\hline 6 & 0.51740800 & 1. 76703700 & 1. 78953200 \\
\hline 1 & 0.78770600 & 1. 99056900 & 2. 82793100 \\
\hline 1 & 0.01964600 & 2. 65781700 & 1. 38894100 \\
\hline 1 & -0.23166500 & 0.97024600 & 1. 81584300 \\
\hline 6 & -3.11772800 & -1.54956500 & 1. 04819500 \\
\hline 1 & -3.15160900 & -1.45853100 & 2. 13998100 \\
\hline 1 & -4.03624000 & -2.06028900 & 0.73898600 \\
\hline 1 & -2.28362700 & -2.21479300 & 0.80639700 \\
\hline 6 & -5.24316000 & 0.41879700 & 1. 35879800 \\
\hline 1 & -5.79098600 & -0.42860900 & 0.93184100 \\
\hline 1 & -4.98496200 & 0.14512900 & 2. 38765900 \\
\hline 1 & -5.92460500 & 1. 27173000 & 1. 39834600 \\
\hline 6 & -2.67031600 & 3. 70004100 & -1.45832800 \\
\hline 1 & -1.79269900 & 4. 25184500 & -1.10273200 \\
\hline 1 & -2.57775300 & 3. 62452800 & -2.54751000 \\
\hline 1 & -3.55568500 & 4. 30041200 & -1.23701200 \\
\hline 6 & -0.52000100 & 1. 76858200 & -1.78831900 \\
\hline 1 & -0.79088600 & 1. 99530400 & -2.82586800 \\
\hline 1 & -0.02159900 & 2. 65800200 & -1.38545600 \\
\hline 1 & 0.22868500 & 0.97154100 & -1.81756800 \\
\hline \multicolumn{4}{|l|}{64} \\
\hline \multicolumn{4}{|l|}{ 1_1 } \\
\hline 6 & 1. 52635200 & 1. 43484900 & -0.56305700 \\
\hline 6 & 1. 77923300 & 2. 59808700 & 0. 18480100 \\
\hline 6 & 3. 09039000 & 3. 08307700 & 0.29858000 \\
\hline 6 & 4. 11887300 & 2. 39841000 & -0.34458700 \\
\hline 1 & 5. 13751500 & 2. 76971000 & -0.24980400 \\
\hline 6 & 3. 88501900 & 1. 26139500 & -1.11532500 \\
\hline 6 & 2.57383600 & 0.77733700 & -1.23587500 \\
\hline 6 & 0.20998400 & -1.79492200 & 0.20377800 \\
\hline 6 & -0.44628200 & -2.70116300 & -0.67264900 \\
\hline 6 & -0.00381400 & -4.03030000 & -0.79419100 \\
\hline 6 & 1. 12424600 & -4.43382300 & -0.09179100 \\
\hline 1 & 1. 45623200 & -5.46738500 & -0.16837600 \\
\hline 6 & 1. 87045600 & -3.53537300 & 0.66278700 \\
\hline 6 & 1. 43592900 & -2.20411500 & 0.78926000 \\
\hline 5 & 0.07320200 & 0.84626300 & -0.59358300 \\
\hline 5 & -0.46331200 & -0.34133600 & 0.51844200 \\
\hline 35 & -1.02201600 & 1. 44152900 & -2.13564700 \\
\hline 35 & -0.17495900 & 0.30768900 & 2.52527700 \\
\hline 6 & 2. 32710100 & -0.45625100 & -2.07141400 \\
\hline 1 & 2. 78139900 & -0.35357600 & -3.06289900 \\
\hline 1 & 1. 26375300 & -0.65764000 & -2.22238800 \\
\hline 1 & 2. 76034700 & -1.35073600 & -1.60655300 \\
\hline 6 & 5. 03010600 & 0.56331700 & -1.80195400 \\
\hline 1 & 4. 92760500 & 0.59565100 & -2.89262900 \\
\hline 1 & 5. 08493200 & -0.49403700 & -1.51966400 \\
\hline
\end{tabular}




\begin{tabular}{|c|c|c|c|}
\hline 1 & 5.98308200 & 1. 03069700 & -1.54287000 \\
\hline 6 & 3. 38572600 & 4. 32545700 & 1. 09718100 \\
\hline 1 & 3. 03580300 & 4. 23328300 & 2. 13106400 \\
\hline 1 & 2. 89036600 & 5. 20572500 & 0.67141900 \\
\hline 1 & 4. 45925000 & 4. 52695000 & 1. 12248400 \\
\hline 6 & 0.65729900 & 3. 35785400 & 0.84982500 \\
\hline 1 & 0.63337200 & 4. 39857600 & 0.50652600 \\
\hline 1 & 0.76720900 & 3. 37204000 & 1. 93923900 \\
\hline 1 & -0.32310200 & 2. 92598300 & 0.62836300 \\
\hline 6 & -1.60453000 & -2.30650000 & -1.57032000 \\
\hline 1 & -2.56534000 & -2.71679300 & -1.22580600 \\
\hline 1 & -1.44756100 & -2.71760900 & -2.57161000 \\
\hline 1 & -1.71183700 & -1.22971400 & -1.69119800 \\
\hline 6 & -0.72028400 & -5.02145200 & -1.67660500 \\
\hline 1 & -0.61534400 & -4.77317600 & -2.73910000 \\
\hline 1 & -1.79368200 & -5.06191800 & -1.46268000 \\
\hline 1 & -0.31258700 & -6.02479300 & -1.53282500 \\
\hline 6 & 3. 14250800 & -4.00796900 & 1. 32080900 \\
\hline 1 & 3. 22859000 & -5.09515400 & 1. 25346100 \\
\hline 1 & 3. 18334600 & -3.72980800 & 2. 37860800 \\
\hline 1 & 4. 03021000 & -3.57623700 & 0.84376500 \\
\hline 6 & 2. 38467100 & -1.26581800 & 1. 50184000 \\
\hline 1 & 2. 17206400 & -0.21758500 & 1. 31534000 \\
\hline 1 & 3. 40662200 & -1.45541800 & 1. 15486700 \\
\hline 1 & 2. 37929100 & -1.41933400 & 2. 58673700 \\
\hline 7 & -2.08532700 & -0.52625100 & 0.57295400 \\
\hline 7 & -2.48218200 & -1.57181200 & 1. 15654400 \\
\hline 7 & -2.75562600 & -2.52994300 & 1. 66148500 \\
\hline 6 & -3.13860000 & 0.44248000 & 0.31731500 \\
\hline 6 & -4.21714400 & 0.06750200 & -0.47550100 \\
\hline 6 & -3.01661100 & 1. 72209600 & 0.84458700 \\
\hline 6 & -5.20339200 & 1. 00980200 & -0.74913600 \\
\hline 6 & -4.00307100 & 2. 65664300 & 0.54674800 \\
\hline 6 & -5.09293300 & 2. 30377600 & -0.24598800 \\
\hline 1 & -4.27139800 & -0.93650100 & -0.88604700 \\
\hline 1 & -2.17293400 & 1.96706200 & 1. 48041700 \\
\hline 1 & -6.05118900 & 0.73390700 & -1.36691900 \\
\hline 1 & -3.92018600 & 3. 66201200 & 0.94555000 \\
\hline 1 & -5.85958300 & 3. 03822700 & -0.46944000 \\
\hline \multicolumn{4}{|c|}{ t. } \\
\hline \multicolumn{4}{|l|}{ 1_2 } \\
\hline 6 & 2. 55821700 & -0.86543300 & -0.22741300 \\
\hline 6 & 3. 62957400 & -0.05706800 & 0.19622300 \\
\hline 6 & 4. 85574300 & -0.64511400 & 0.53714200 \\
\hline 6 & 4.98670200 & -2.02813500 & 0.44562100 \\
\hline 1 & 5.93766600 & -2.48549000 & 0.71237900 \\
\hline 6 & 3. 94286800 & -2.84640300 & 0.02095900 \\
\hline 6 & 2.71364000 & -2.26309200 & -0.32321500 \\
\hline 6 & -3.30683200 & 0.45432200 & 0.66226700 \\
\hline 6 & -3.32057800 & 0.78998000 & -0.70085800 \\
\hline 6 & -3.93493900 & -0.10628100 & -1.59060200 \\
\hline 6 & -4.52157100 & -1.26928400 & -1.10010400 \\
\hline 1 & -4.97697300 & -1.96314600 & -1.80314700 \\
\hline 6 & -4.56989900 & -1.56035000 & 0.26142300 \\
\hline 6 & -3.97853800 & -0.67355200 & 1. 16820400 \\
\hline 5 & 1. 18021400 & -0.20660900 & -0.56744100 \\
\hline 5 & -0.07511000 & 0.20804100 & 0.49726400 \\
\hline 35 & 0.66596200 & -0.06483500 & -2.46635100 \\
\hline 35 & -0.71365700 & -1.39260800 & 1. 43906800 \\
\hline 6 & 1. 60108000 & -3.16993600 & -0.79159900 \\
\hline 1 & 1.88525800 & -3.68994100 & -1.71390400 \\
\hline 1 & 0.66910900 & -2.64067900 & -0.99253100 \\
\hline 1 & 1. 37915300 & -3.93853900 & -0.04328700 \\
\hline 6 & 4. 13978000 & -4.33754500 & -0.06174100 \\
\hline 1 & 3. 95213200 & -4.71592500 & -1.07246200 \\
\hline 1 & 3. 45849000 & -4.87182900 & 0.60992500 \\
\hline 1 & 5. 16142400 & -4.61002400 & 0.21286700 \\
\hline 6 & 6. 01505900 & 0.20024800 & 0.99498100 \\
\hline
\end{tabular}

\begin{tabular}{|c|c|c|c|}
\hline 1 & 5. 74437100 & 0.82596900 & 1. 85227900 \\
\hline 1 & 6. 35939600 & 0.87489300 & 0.20291600 \\
\hline 1 & 6. 86074700 & -0.42548100 & 1. 28935000 \\
\hline 6 & 3. 48809300 & 1. 44191700 & 0.28476000 \\
\hline 1 & 4. 33166200 & 1. 94976000 & -0.19396000 \\
\hline 1 & 3. 45819600 & 1. 78383000 & 1. 32604100 \\
\hline 1 & 2. 58298500 & 1. 80649200 & -0.20750600 \\
\hline 6 & -2.77575200 & 2. 08434000 & -1.23658800 \\
\hline 1 & -2.48480800 & 2. 78379500 & -0.45221900 \\
\hline 1 & -3.52723400 & 2. 57964600 & -1.85969400 \\
\hline 1 & -1.90408800 & 1.89735300 & -1.87295000 \\
\hline 6 & -3.96993700 & 0.19062200 & -3.06674800 \\
\hline 1 & -2.96588500 & 0.36566300 & -3.46760500 \\
\hline 1 & -4.56287600 & 1. 08604200 & -3.28465200 \\
\hline 1 & -4.41175600 & -0.64264400 & -3.61748100 \\
\hline 6 & -5.24900300 & -2.81259900 & 0.74812500 \\
\hline 1 & -5.57871400 & -3.42675900 & -0.09285000 \\
\hline 1 & -6.12850500 & -2.58180000 & 1. 35949100 \\
\hline 1 & -4.57863900 & -3.41812100 & 1. 36745200 \\
\hline 6 & -4.03391800 & -0.96322600 & 2. 64434200 \\
\hline 1 & -3.40730000 & -1.82463100 & 2. 90214600 \\
\hline 1 & -5.05661400 & -1.20791600 & 2. 94781200 \\
\hline 1 & -3.69262200 & -0.11405600 & 3. 23641800 \\
\hline 7 & -0.62831300 & 1. 47800800 & 0.75428700 \\
\hline 7 & -1.64341200 & 1. 79888400 & 1. 74520500 \\
\hline 7 & -2.77043000 & 1. 32435700 & 1. 66417100 \\
\hline 6 & 0.19266200 & 2. 65174300 & 0.56590400 \\
\hline 6 & 0.42490500 & 3. 16913700 & -0.70559600 \\
\hline 6 & 0.77324600 & 3. 24848000 & 1. 68741500 \\
\hline 6 & 1. 27152400 & 4. 26698700 & -0.85681500 \\
\hline 6 & 1. 60034900 & 4. 35306700 & 1. 52813600 \\
\hline 6 & 1. 85995300 & 4. 85984700 & 0.25478800 \\
\hline 1 & -0.02747000 & 2. 70305800 & -1.57138200 \\
\hline 1 & 0.57206000 & 2. 83459000 & 2. 66982800 \\
\hline 1 & 1. 46388100 & 4. 65752000 & -1.85090600 \\
\hline 1 & 2. 05491700 & 4. 81153400 & 2. 40057000 \\
\hline 1 & 2. 51633500 & 5. 71537900 & 0.13314400 \\
\hline \multicolumn{4}{|r|}{ 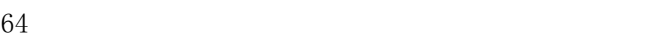 } \\
\hline \multicolumn{4}{|l|}{ 1_3 } \\
\hline 6 & 1. 31707700 & 0.11814500 & -0.45479800 \\
\hline 6 & 2. 63120400 & -0.26473600 & -0.86419100 \\
\hline 6 & 3. 75193200 & 0.39690200 & -0.33787300 \\
\hline 6 & 3. 56182900 & 1. 46646300 & 0.53217400 \\
\hline 1 & 4. 43628300 & 1.97123500 & 0.93877200 \\
\hline 6 & 2. 29973500 & 1. 92871100 & 0.88445400 \\
\hline 6 & 1. 16889400 & 1. 26171200 & 0.38724900 \\
\hline 6 & -1.64330500 & 1. 61367800 & -2.48431100 \\
\hline 6 & -0.30042200 & 1. 83092600 & -2.81862200 \\
\hline 6 & 0.30314200 & 3. 02363100 & -2.39696200 \\
\hline 6 & -0.47475400 & 3. 98428300 & -1.75522400 \\
\hline 1 & -0.00650200 & 4. 91496700 & -1.44099900 \\
\hline 6 & -1.83701800 & 3. 80405000 & -1.51770700 \\
\hline 6 & -2.44776100 & 2. 59367200 & -1.87783500 \\
\hline 5 & 0.02611400 & -0.70432700 & -0.74268200 \\
\hline 5 & -1.55839700 & -0.57581400 & -0.17674200 \\
\hline 35 & -0.02763400 & -2.50923200 & -1.57877900 \\
\hline 35 & -1.64942600 & -1.22382800 & 1.69541700 \\
\hline 6 & -0.16861600 & 1. 82225000 & 0.80461300 \\
\hline 1 & -0.46824100 & 1. 44586700 & 1. 78905100 \\
\hline 1 & -0.96579500 & 1. 60611800 & 0.09261500 \\
\hline 1 & -0.11735800 & 2. 91036400 & 0.87421000 \\
\hline 6 & 2. 18277100 & 3. 13076900 & 1. 78729700 \\
\hline 1 & 1. 54939000 & 2. 92975600 & 2. 65690100 \\
\hline 1 & 1. 74746800 & 3. 98853600 & 1. 26118300 \\
\hline 1 & 3. 16760100 & 3. 43229900 & 2. 15114300 \\
\hline 6 & 5. 16259100 & -0.01473300 & -0.68339900 \\
\hline 1 & 5. 41222700 & 0.20931900 & -1.72649500 \\
\hline 1 & 5. 32170500 & -1.08685300 & -0.53368000 \\
\hline
\end{tabular}




\begin{tabular}{|c|c|c|c|}
\hline 1 & 5. 87712300 & 0.52041000 & -0.05380800 \\
\hline 6 & 2. 90718900 & -1.35477900 & -1.87309400 \\
\hline 1 & 3. 85825400 & -1.17194800 & -2.37572400 \\
\hline 1 & 2. 14039500 & -1.41489200 & -2.64233100 \\
\hline 1 & 2. 97215100 & -2.34161600 & -1.40095900 \\
\hline 6 & 0.48281200 & 0.83292200 & -3.63126000 \\
\hline 1 & -0.02396000 & -0.13033200 & -3.71497500 \\
\hline 1 & 1. 47265000 & 0.66905300 & -3.19815300 \\
\hline 1 & 0.63170800 & 1. 21457700 & -4.64846900 \\
\hline 6 & 1. 77172600 & 3. 25202200 & -2.63022700 \\
\hline 1 & 2. 02338600 & 3. 21543700 & -3.69591000 \\
\hline 1 & 2. 36816700 & 2. 47946100 & -2.12795100 \\
\hline 1 & 2. 08320900 & 4. 22436300 & -2.24163500 \\
\hline 6 & -2.64001900 & 4. 89411300 & -0.85832800 \\
\hline 1 & -1.99687100 & 5. 72556400 & -0.56158900 \\
\hline 1 & -3.15664200 & 4. 52965400 & 0.03604400 \\
\hline 1 & -3.40771700 & 5. 29104800 & -1.53201400 \\
\hline 6 & -3.92267500 & 2. 40351500 & -1.64563000 \\
\hline 1 & -4.47752000 & 3. 27902200 & -1.99632100 \\
\hline 1 & -4.14634900 & 2. 28954200 & -0.57962500 \\
\hline 1 & -4.32460200 & 1. 53092700 & -2.16228300 \\
\hline 7 & -2.77425300 & -0.39130900 & -0.88056100 \\
\hline 7 & -2.78535400 & -0.45820000 & -2.33379700 \\
\hline 7 & -2.24161300 & 0.41463300 & -2.99800600 \\
\hline 6 & -4.05271000 & -0.84240500 & -0.39315200 \\
\hline 6 & -4.67400400 & -0.15867000 & 0.64906700 \\
\hline 6 & -4.65476600 & -1.96661300 & -0.95810800 \\
\hline 6 & -5.90130500 & -0.60533500 & 1. 13126800 \\
\hline 6 & -5.88829000 & -2.39554300 & -0.47982000 \\
\hline 6 & -6.51413500 & -1.71959600 & 0.56491800 \\
\hline 1 & -4.18415200 & 0.70366400 & 1. 09000900 \\
\hline 1 & -4.15360800 & -2.49201500 & -1.76271300 \\
\hline 1 & -6.37916600 & -0.07699500 & 1. 94999700 \\
\hline 1 & -6.35510700 & -3.27058100 & -0.92063600 \\
\hline 1 & -7.47343600 & -2.06298900 & 0.93828800 \\
\hline \multicolumn{4}{|c|}{ (1) } \\
\hline \multicolumn{4}{|l|}{$1 \_4$} \\
\hline 6 & 0.40299600 & -1.62331600 & 0.73296800 \\
\hline 6 & -0.43113100 & -0.87248400 & 1. 58705400 \\
\hline 6 & -1.76329100 & -1.26684000 & 1. 80954000 \\
\hline 6 & -2.21738600 & -2.46728100 & 1. 26351400 \\
\hline 1 & -3.23778200 & -2.78756300 & 1. 46872500 \\
\hline 6 & -1.39501800 & -3.27917100 & 0.48531500 \\
\hline 6 & -0.09148200 & -2.84037600 & 0.19134300 \\
\hline 6 & -2.70824100 & 0.57160300 & -0.71328000 \\
\hline 6 & -4.02173700 & 0.10325900 & -0.54824400 \\
\hline 6 & -4.93576900 & 1.03915300 & -0.05310700 \\
\hline 6 & -4.49821400 & 2. 33537300 & 0.22943300 \\
\hline 1 & -5.22411600 & 3. 04845800 & 0.61285000 \\
\hline 6 & -3.18344800 & 2. 76956300 & 0.04526900 \\
\hline 6 & -2.23222500 & 1. 86605000 & -0.44317800 \\
\hline 5 & 1.78974900 & -1.05251500 & 0.17758100 \\
\hline 5 & 1.88791500 & -0.18148100 & -1.24111500 \\
\hline 35 & 3. 45464900 & -2.13559700 & 0.84472900 \\
\hline 35 & 1.99902600 & 0.49446400 & -3.07472500 \\
\hline 6 & 0.77429100 & -3.66573700 & -0.72607100 \\
\hline 1 & 1. 45005400 & -4.30982900 & -0.15226900 \\
\hline 1 & 1. 40605700 & -3.01971400 & -1.34095500 \\
\hline 1 & 0.18101900 & -4.30424800 & -1.38497800 \\
\hline 6 & -1.94002600 & -4.57890500 & -0.05428100 \\
\hline 1 & -1.30159100 & -5.42659000 & 0.21347400 \\
\hline 1 & -2.01441600 & -4.57248100 & -1.14830200 \\
\hline 1 & -2.93906700 & -4.77287400 & 0.34532400 \\
\hline 6 & -2.71222500 & -0.41268500 & 2. 61093900 \\
\hline 1 & -2.71644100 & 0.62558200 & 2. 25458100 \\
\hline 1 & -2.44261000 & -0.38477600 & 3. 67270000 \\
\hline 1 & -3.73358800 & -0.79922600 & 2. 54281300 \\
\hline 6 & 0.08884600 & 0.35976300 & 2. 28738600 \\
\hline
\end{tabular}

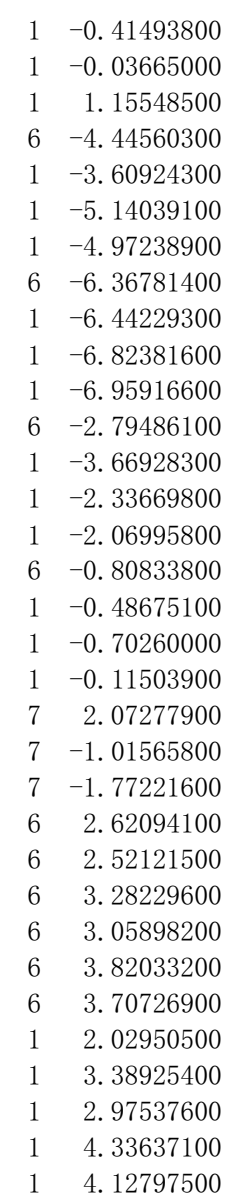

0. 51462500

1. 28011300

0. 25914100

$-1.29422100$

$-1.94814900$

$-1.27836300$

$-1.74746000$

0.64368200

$-0.12912700$

0. 23918700

1. 50158600

4. 18761900

4. 76857200

4. 67941500

4. 22331900

2. 25479700

3. 04454800

2. 63532700

1. 41845800

0. 44075800

$-1.01075600$

$-0.32937400$

1. 58603900

2. 81910400

1. 52092200

3. 96586300

2. 67300200

3. 90132300

2. 85962300

0. 55514400

4. 91515400

2. 60793300

4. 79727100

64

$6-0.46447500$

2. 07052900

1. 69237300

2. 19710300

3. 02584700

3. 40669200

3. 39474900

2. 92125200

$-2.13170000$

$-1.34691400$

$-1.34280000$

$-2.10807700$

$-2.08468500$

$-2.91215000$

$-2.93980100$

1. 54504000

0. 49369700

2. 12781000

1. 00401500

3. 32550100

4. 36664200

3. 23744100

2. 71037100

4. 29142300

5. 27139200

3. 86116100

4. 45740100

1. 87211400

0. 79218500

2. 23411300

2. 33743600

0. 75183100

1. 29090800

0.00083900
3. 24511400

1. 70228400

2. 48845500

$-0.89098100$

$-1.14155900$

$-1.73764100$

$-0.04615000$

0. 17965300

0.95222600

$-0.72940900$

0.50349300

0. 36518500

0. 66291100

$-0.49805800$

1. 18484000

$-0.71054900$

$-0.02984100$

$-1.73399800$

$-0.58512300$

$-0.03233500$

$-1.66321700$

$-1.22635200$

0. 54771200

$-0.11140800$

1. 78007900

0. 46301200

2. 34369600

1. 69479500

$-1.07922900$

2. 26450200

$-0.05825100$

3. 29697700

2. 13979300

$-0.12539600$

$-0.60357700$

0. 02600400

1. 13859400

1. 62873000

1. 63771500

0. 98892700

0. 21494200

1. 12697600

1. 02081000

0. 02670900

$-0.05922900$

$-0.85188700$

$-0.75086100$

$-0.79682300$

$-0.16247600$

$-2.62585000$

0. 07133800

1. 49602500

1. 83015900

0. 72697600

2. 34921600

2. 84525600

2. 58789400

3. 61825100

3. 28551200

$-0.49746500$

$-0.51574800$

$-1.52396300$

0. 11974300

$-1.77228300$

$-2.67494100$

$-1.55521700$ 


\begin{tabular}{|c|c|c|c|}
\hline 1 & -0.98822500 & 0.21059300 & -2.01006100 \\
\hline 6 & -1.64771100 & -0.54178200 & 2. 20577600 \\
\hline 1 & -1.54285200 & 0.50986100 & 1. 91249800 \\
\hline 1 & -0.65139900 & -0.92631600 & 2. 43161300 \\
\hline 1 & -2.23089700 & -0.57056700 & 3. 12984900 \\
\hline 6 & -4.56202800 & -0.51452100 & 1. 96436800 \\
\hline 1 & -4.18719400 & 0.51283800 & 2. 03012400 \\
\hline 1 & -4.54639600 & -0.92861100 & 2. 97930400 \\
\hline 1 & -5.60392900 & -0.47973100 & 1. 63669600 \\
\hline 6 & -4.32060600 & -3.73035300 & -1.90041400 \\
\hline 1 & -5.39241000 & -3.51998900 & -1.90112700 \\
\hline 1 & -4.19085000 & -4.80415100 & -1.72510200 \\
\hline 1 & -3.93325800 & -3.52058900 & -2.90306500 \\
\hline 6 & -1.38055300 & -3.76232400 & -1.69692300 \\
\hline 1 & -0.37645500 & -3.92324700 & -1.29936100 \\
\hline 1 & -1.27715200 & -3.26306600 & -2.66807900 \\
\hline 1 & -1.83798900 & -4.73827000 & -1.88005900 \\
\hline 7 & 1.62840200 & -0.83348300 & 0.14223800 \\
\hline 7 & 0.25774700 & -1.00074600 & 0.00701800 \\
\hline 7 & -0.17290000 & -2.12571000 & 0.27640200 \\
\hline 6 & 2. 44552900 & -1.92702400 & 0.59213500 \\
\hline 6 & 2. 72724100 & -2.05693900 & 1. 94799400 \\
\hline 6 & 2. 94225000 & -2.83614100 & -0.33694600 \\
\hline 6 & 3. 52475300 & -3.11265100 & 2. 37867900 \\
\hline 6 & 3.73815600 & -3.88952700 & 0.10140700 \\
\hline 6 & 4. 02911700 & -4.02751300 & 1. 45696300 \\
\hline 1 & 2. 32972900 & -1.32926900 & 2. 64913900 \\
\hline 1 & 2. 70538600 & -2.70654000 & -1.38864100 \\
\hline 1 & 3. 75462200 & -3.21868900 & 3. 43381800 \\
\hline 1 & 4. 13422200 & -4.60035700 & -0.61637500 \\
\hline 1 & 4. 65278000 & -4.84880300 & 1. 79509500 \\
\hline \multicolumn{4}{|l|}{64} \\
\hline \multicolumn{4}{|l|}{ 1_P } \\
\hline 6 & -1.50859800 & -1.35701800 & 0.12168200 \\
\hline 6 & -2.40388900 & -1.04616900 & 1. 16634500 \\
\hline 6 & -3.61846100 & -1.74051300 & 1. 27579100 \\
\hline 6 & -3.91472800 & -2.73429500 & 0.34666700 \\
\hline 1 & -4.85684200 & -3.27249300 & 0.43488400 \\
\hline 6 & -3.04698500 & -3.06282000 & -0.69183000 \\
\hline 6 & -1.83361500 & -2.36801600 & -0.80670600 \\
\hline 6 & -1.17510400 & 1. 80245400 & -0.44779300 \\
\hline 6 & -1.27617700 & 2.81279400 & 0.51998600 \\
\hline 6 & -2.36043700 & 3. 69847300 & 0.43494300 \\
\hline 6 & -3.29307200 & 3. 53419600 & -0.58753900 \\
\hline 1 & -4.13962000 & 4. 21539000 & -0.63810500 \\
\hline 6 & -3.17725200 & 2. 53743800 & -1.55310500 \\
\hline 6 & -2.08810300 & 1. 65432800 & -1.49920600 \\
\hline 5 & -0.15261500 & -0.59553700 & -0.03513000 \\
\hline 5 & 1.50003500 & -0.91420200 & 0.16876400 \\
\hline 35 & 2. 52689200 & -2.38783800 & -0.71011400 \\
\hline 35 & 1. 61231900 & -0.92842500 & 2. 24244000 \\
\hline 6 & -0.92807800 & -2.69538600 & -1.96854800 \\
\hline 1 & -1.45222500 & -2.56990500 & -2.92364000 \\
\hline 1 & -0.58304000 & -3.73444200 & -1.92750900 \\
\hline 1 & -0.03672800 & -2.06581300 & -1.99626200 \\
\hline 6 & -3.41530200 & -4.14372500 & -1.67428500 \\
\hline 1 & -2.65957500 & -4.93616900 & -1.70191800 \\
\hline 1 & -3.50271000 & -3.75157600 & -2.69402300 \\
\hline 1 & -4.37151700 & -4.60080900 & -1.40927300 \\
\hline 6 & -4.59122500 & -1.42417000 & 2. 38159000 \\
\hline 1 & -4.86798800 & -0.36429100 & 2. 38397000 \\
\hline 1 & -4.16622600 & -1.64342800 & 3. 36774800 \\
\hline 1 & -5.50711200 & -2.01015300 & 2. 27521100 \\
\hline 6 & -2.09457800 & 0.00910100 & 2. 19884500 \\
\hline 1 & -2.17439600 & -0.40348100 & 3. 21056100 \\
\hline 1 & -2.79838800 & 0.84791200 & 2. 13166000 \\
\hline 1 & -1.08362800 & 0.40541200 & 2. 10192600 \\
\hline 6 & -0.27192100 & 2.96854800 & 1. 63407200 \\
\hline
\end{tabular}

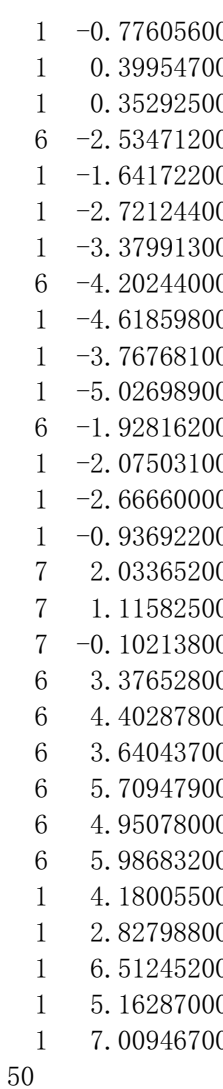

3. 16884100

1_P1

$6-1.45598900$

$\begin{array}{ll}6 & -1.45090500\end{array}$

$\begin{array}{ll}6 & -2.64840300\end{array}$

$\begin{array}{ll}6 & -3.84952000\end{array}$

$1-4.77258700$

$\begin{array}{ll}6 & -3.90937200\end{array}$

$\begin{array}{ll}6 & -2.71476300\end{array}$

$\begin{array}{ll}6 & -1.42552200\end{array}$

$\begin{array}{ll}6 & -2.30597600\end{array}$

$\begin{array}{ll}6 & -3.50840800\end{array}$

$\begin{array}{ll}6 & -3.74327300\end{array}$

$1-4.67612100$

$\begin{array}{ll}6 & -2.82229700\end{array}$

$6-1.63951300$

$5-0.10348700$

$5 \quad 1.47425400$

$35 \quad 2.94637200$

$35 \quad 1.43469700$

$\begin{array}{ll}6 & -2.86059200\end{array}$

$1-3.45763900$

$1-3.38388900$

$1-1.90211400$

$\begin{array}{ll}6 & -5.24251400\end{array}$

$1-5.44946700$

$1-5.28071700$

$1-6.05364000$

$\begin{array}{ll}6 & -2.64622300\end{array}$

$1-2.33241700$

$1-3.64457400$

$1-1.95728500$

$\begin{array}{ll}6 & -0.21935700\end{array}$

$1-0.42481500$

10.06168400

10.64365700

$\begin{array}{ll}6 & -2.01697800\end{array}$

$1-2.67661000$

$1-2.18549400$
3. 81217000

2. 08474900

4. 79661100

5. 42593700

4. 38904400

5. 43678300

2. 42017000

1. 40900100

2. 64250200

3. 11739300

0. 60200800

1. 03807100

$-0.19821400$

0. 14542300

0. 51882200

1. 36559700

0. 85010200

0.97648700

0. 34938400

2. 02043200

0. 79020200

2. 45038900

1. 83767100

$-0.46125600$

2. 47781100

0. 30763400

3. 26003700

2. 17275000

2. 58370600

1. 43699100

1. 77765600

1. 45097800

1. 52355500

2. 45107900

1. 18922400

$-2.64968300$

$-2.70489400$

$-3.63079600$

$-2.48568100$

$-2.56382900$

$-3.55663000$

$-2.44250400$

$-2.54810300$

$-0.25379400$

$-0.46598700$

$-0.32707900$

$-0.44181000$

0. 26208200

$-1.32885900$

0. 08157900

$-1.49495800$

$-0.79237900$

0.94571200

$-1.88183800$

0. 62837700

$-2.18533300$

$-0.93147400$

1. 14625900

2. 07228500

2. 40618600

1. 88169100

2. 11975200

1. 13367000

0. 79830800

$-2.04811400$

$-2.12610700$

$-2.83018800$

$-3.47781500$

$-4.02273400$

$-3.46683600$

$-2.73006100$

0. 38195400

0. 29040400

1. 57949900

0.11913500

0. 17747700

$-0.73954300$

0.88438800

$-0.04338700$

0.69117400

1. 13565600

$-0.39688700$

0. 97869900

3. 34468200

4. 35416700

3. 42008100

3. 01141900

2. 84583600

3. 91546900

2. 69829500

2. 60809900

$-1.49791500$

$-0.64292900$

$-2.22774900$
-0. 42437400

$-1.49594500$

$-2.15557500$

$-1.69053300$

$-2.21650900$

$-0.51672600$

0. 13809300

0. 30827400

$-0.77701800$

$-0.59417200$

0.61484700

0. 74333000

1. 66359800

1. 51641700

$-0.00815400$

$-0.75940500$

$-0.15905100$

$-2.82920700$

1. 50849000

1. 49225100

2. 16683600

1. 97593000

0. 03022100

1. 01076600

0. 16326400

$-0.64309700$

$-3.33416700$

$-3.04416300$

$-3.77194000$

$-4.11709600$

$-1.89296400$

$-1.75882200$

$-2.93886800$

$-1.27490000$

$-2.11285600$

$-2.30174300$

$-2.91145400$ 


\begin{tabular}{|c|c|c|c|}
\hline 1 & -0.98862300 & -1.14878800 & -2.19747700 \\
\hline 6 & -4.52970000 & -2.88330000 & -1.69912100 \\
\hline 1 & -4.16461100 & -3.45771200 & -2.55789900 \\
\hline 1 & -4.76799400 & -1.87863200 & -2.06579900 \\
\hline 1 & -5.45453400 & -3.34999300 & -1.35218700 \\
\hline 6 & -3.13978700 & -4.21276900 & 2. 93643100 \\
\hline 1 & -3.17505600 & -3.54472800 & 3. 80330700 \\
\hline 1 & -2.39508400 & -4.98643300 & 3. 15016200 \\
\hline 1 & -4.11181600 & -4.70436500 & 2. 85649500 \\
\hline 6 & -0.65874200 & -2.60453700 & 2. 65321400 \\
\hline 1 & -0.70273700 & -1.60181300 & 3. 09945300 \\
\hline 1 & 0.36819400 & -2.78513700 & 2. 32664800 \\
\hline 1 & -0.87672800 & -3.31697900 & 3. 44854200 \\
\hline 7 & 1. 85430300 & -1.17053400 & -0.28231200 \\
\hline 7 & 0.84150400 & -1.87527800 & 0.05980900 \\
\hline 7 & -0.26057800 & -1.19488600 & 0.17608400 \\
\hline 6 & 3. 10886800 & -1.85407200 & -0.27326500 \\
\hline 6 & 4. 12312000 & -1.47098900 & -1.15090900 \\
\hline 6 & 3.31972100 & -2.87868600 & 0.65200900 \\
\hline 6 & 5. 34249900 & -2.13856900 & -1.10856000 \\
\hline 6 & 4. 54122700 & -3.54094000 & 0.67725800 \\
\hline 6 & 5.55667400 & -3.17302900 & -0.20111500 \\
\hline 1 & 3.95363400 & -0.67321000 & -1.86299100 \\
\hline 1 & 2.52995400 & -3.14891500 & 1. 34476300 \\
\hline 1 & 6. 12980700 & -1.84428800 & -1.79476400 \\
\hline 1 & 4. 70038500 & -4.33731300 & 1. 39685400 \\
\hline 1 & 6.51257900 & -3.68588400 & -0.17419800 \\
\hline 7 & 0.29824800 & 0.91113300 & 1. 70325700 \\
\hline 7 & 1. 14812000 & 0.18290100 & 2. 27151200 \\
\hline 7 & 1.87694700 & -0.53290700 & 2. 73447600 \\
\hline 6 & 0.28614400 & 2. 27657800 & 2. 19935500 \\
\hline 6 & 1. 42524600 & 2. 82991900 & 2. 78301900 \\
\hline 6 & -0.88996400 & 3. 01019900 & 2. 08170300 \\
\hline 6 & 1. 37947400 & 4. 13586000 & 3. 25606900 \\
\hline 6 & -0.91343800 & 4. 32155000 & 2.54836500 \\
\hline 6 & 0.21343300 & 4. 88733500 & 3. 13614400 \\
\hline 1 & 2. 34727600 & 2. 26059700 & 2. 84547500 \\
\hline 1 & -1.76788500 & 2.56921700 & 1. 63112300 \\
\hline 1 & 2. 26700600 & 4. 56797100 & 3. 70592200 \\
\hline 1 & -1.82856600 & 4. 89669400 & 2. 45407700 \\
\hline 1 & 0.18474600 & 5. 90941400 & 3. 49851100 \\
\hline \multicolumn{4}{|c|}{78} \\
\hline \multicolumn{4}{|c|}{ 1_P2 } \\
\hline 6 & 2. 01914400 & -0.95888000 & 0.19496300 \\
\hline 6 & 2.51018100 & -1.85366600 & -0.77830200 \\
\hline 6 & 3.82993500 & -2.32611100 & -0.68655900 \\
\hline 6 & 4. 62752900 & -1.90920500 & 0.37579500 \\
\hline 1 & 5. 65175400 & -2.27240000 & 0.43903800 \\
\hline 6 & 4. 14995800 & -1.06198400 & 1. 37239000 \\
\hline 6 & 2. 83164100 & -0.59384400 & 1. 28543700 \\
\hline 6 & 1. 48888700 & 2. 01263600 & -0.35397300 \\
\hline 6 & 2.31464600 & 1. 90551300 & -1.48006200 \\
\hline 6 & 3. 41752500 & 2. 76810800 & -1.57449500 \\
\hline 6 & 3. 62659800 & 3. 71687000 & -0.57810500 \\
\hline 1 & 4. 47945600 & 4. 38661900 & -0.66266500 \\
\hline 6 & 2. 77273300 & 3.85275500 & 0.51306800 \\
\hline 6 & 1. 67676400 & 2.98552500 & 0.64069600 \\
\hline 5 & 0.56184700 & -0.37961000 & 0.04061100 \\
\hline 5 & -1.89899100 & -0.52681300 & -0.40637800 \\
\hline 35 & -3.57492500 & -1.28175200 & 0.44313500 \\
\hline 35 & -2.00033200 & -0.73499700 & -2.51343100 \\
\hline 6 & 2. 29512100 & 0.25436100 & 2. 41018300 \\
\hline 1 & 2. 84490500 & 1. 19699100 & 2. 51333700 \\
\hline 1 & 2. 38217400 & -0.27142500 & 3. 36886300 \\
\hline 1 & 1. 23821900 & 0.49652400 & 2. 27324700 \\
\hline 6 & 5. 03654900 & -0.66391100 & 2. 52278900 \\
\hline 1 & 4. 63107300 & -1.00234600 & 3. 48304300 \\
\hline 1 & 5. 14548200 & 0.42439300 & 2. 58803800 \\
\hline
\end{tabular}

$\begin{array}{lll}1 & 6.03462300 & -1.09434400 \\ 6 & 4.38510900 & -3.26797900 \\ 1 & 4.39753800 & -2.81137900 \\ 1 & 3.78588900 & -4.18149000 \\ 1 & 5.40923800 & -3.55730100 \\ 6 & 1.65732300 & -2.34566200 \\ 1 & 1.47156400 & -3.42203000 \\ 1 & 2.15505800 & -2.19454800 \\ 1 & 0.68441500 & -1.85271300\end{array}$

$\begin{array}{lll}6 & 2.04840200 & 0.92857600\end{array}$

$1 \quad 2.81126900 \quad 0.14330100$

$12.08195200 \quad 1.44196600$

$1 \quad 1.06886500 \quad 0.45254100$

$6 \quad 4.35244300 \quad 2.68473800$

$1 \quad 3.84357900 \quad 2.94179000$

$14.75661900 \quad 1.67436500$

$1 \quad 5.19158900 \quad 3.37280700$

$6 \quad 3.03548000 \quad 4.91644400$

$1 \quad 3.27829200 \quad 4.47814000$

$1 \quad 2.16374800 \quad 5.56261800$

$13.87610200 \quad 5.54666000$

$6 \quad 0.74641700 \quad 3.16167400$

$1 \quad 0.09160900 \quad 4.02615400$

$1 \quad 1.30979700 \quad 3.34780600$

$1 \quad 0.10647100 \quad 2.29862300$

$7-1.84520000 \quad 1.02920200$

$7-0.77042900 \quad 1.70794400$

$7 \quad 0.37755600 \quad 1.08512200$

$\begin{array}{lll}6 & -3.01275900 & 1.86631500\end{array}$

$6-4.09387100 \quad 1.74964900$

$\begin{array}{lll}6 & -3.01591000 & 2.78785500\end{array}$

$\begin{array}{lll}6 & -5.18974700 & 2.58538800\end{array}$

$6-4.12014600 \quad 3.61589400$

$6-5.20735400 \quad 3.51487900$

$1-4.07070700 \quad 1.02370200$

$1 \quad-2.16312900 \quad 2.84182100$

$1-6.03496700 \quad 2.50750300$

$1-4.13037300 \quad 4.33344700$

$1-6.07029300 \quad 4.15845700$

$7 \quad-0.63196700 \quad-1.11761300$

$7-1.39454200 \quad 0.34535600$

$7-1.68272700 \quad-0.49998400$

$6-0.57249700 \quad-2.48542800$

$6 \quad 0.02026600 \quad-2.79603200$

$6-1.09195800 \quad-3.50987500$

$6 \quad 0.10550300 \quad-4.11857200$

$6-1.00874800 \quad-4.82996100$

$6-0.40897000 \quad-5.14050900$

$1 \quad 0.41713400 \quad-1.99497000$

$1-1.55556500 \quad-3.26910000$

$1 \quad 0.57250900 \quad-4.34581800$

$1-1.41538400 \quad-5.61936800$

$1-0.34682300-6.17224200$ 64

\section{1_1'}

$6 \quad 2.36333200$

$6 \quad 2.88865600$

64.22350100

65.02282400

16.05849800

64.54416300

63.20347100

$6-1.90373300$

$6-2.32322800$

$6-3.68609800$

$6-4.62308400$

$1-5.67662300$

$\begin{array}{ll}1 & -4.25403300\end{array}$
2. 41347200

$-1.72300900$

$-2.71948500$

$-1.80137700$

-1. 47645900

$-1.92282500$

$-1.82629000$

$-2.88719200$

$-1.97644500$

$-2.59321300$

$-2.61549000$

$-3.55942900$

$-2.51405100$

$-2.75137600$

$-3.68716800$

$-2.87236300$

$-2.62837000$

1. 54720500

2. 52213700

1. 69363400

1. 24908700

1. 81410400

1. 65112300

2. 73260200

1. 99171800

$-0.19270300$

$-0.19565300$

$-0.23654200$

$-0.02817700$

$-0.89738900$

1. 01436400

$-0.71433900$

1. 18665400

0. 32390600

$-1.70222300$

1. 68295700

$-1.38997400$

2. 00051000

0. 46129100

0. 12757900

2. 77707200

3. 41677200

0. 55210500

1. 77652100

$-0.24184800$

2. 19938900

0. 18904200

1. 40728100

2. 39290600

-1. 19317600

3. 15243200

$-0.43518600$

1. 73809600

$-0.25229000$

$-0.49933800$

$-0.60764400$

$-0.24813200$

1. 29912100

0. 23595600

0. 43041700

0. 16237500

0. 43427000

0. 21475500

$-0.11308900$

$-1.31477400$

$-0.93067500$

$-1.97180300$

$-2.15630800$

$-1.32787900$

$-1.44820700$

$-0.39443600$
0. 45687800

$-0.40230500$

$-0.69124000$

$-0.08525200$

$-0.33087800$

0. 87904100 


\begin{tabular}{|c|c|c|c|}
\hline 6 & -2.89423800 & -0.22294800 & 1. 18486700 \\
\hline 5 & 0.83516000 & -0.54662300 & -0.74643900 \\
\hline 5 & -0.34654300 & -0.54736300 & 0.53657800 \\
\hline 35 & 0.30770800 & -0.62398800 & -2.65127200 \\
\hline 35 & 0.50361400 & -1.35815400 & 2. 25424600 \\
\hline 6 & 2. 70110400 & -2.73661500 & -0.09493900 \\
\hline 1 & 2. 90458000 & -3.22995800 & 0.86037000 \\
\hline 1 & 3. 18339000 & -3.32875800 & -0.88210300 \\
\hline 1 & 1. 62317700 & -2.79980000 & -0.26021500 \\
\hline 6 & 5. 45576600 & -2.19752000 & 0.62435300 \\
\hline 1 & 5. 49983800 & -2.97881600 & -0.14220200 \\
\hline 1 & 5. 11262500 & -2.67618300 & 1. 54824900 \\
\hline 1 & 6.47233400 & -1.83457000 & 0.79312100 \\
\hline 6 & 4. 79511300 & 2. 69178600 & -0.31180600 \\
\hline 1 & 4. 22438100 & 3. 39163900 & 0.30936300 \\
\hline 1 & 4. 78311200 & 3. 08963100 & -1.33278300 \\
\hline 1 & 5. 82992000 & 2. 70257700 & 0.03766800 \\
\hline 6 & 2. 06698700 & 2. 21709800 & -1.11056300 \\
\hline 1 & 2.58233600 & 2. 72184400 & -1.93465300 \\
\hline 1 & 1. 91333800 & 2. 98271600 & -0.33735600 \\
\hline 1 & 1. 08843700 & 1. 91973500 & -1.48996900 \\
\hline 6 & -1.36619200 & -3.00373500 & -0.94592100 \\
\hline 1 & -1.36310800 & -3.05350900 & -2.03874500 \\
\hline 1 & -1.65965000 & -3.99397100 & -0.57736400 \\
\hline 1 & -0.34385500 & -2.83811800 & -0.61040600 \\
\hline 6 & -4.13697600 & -3.24436500 & -1.63059400 \\
\hline 1 & -3.88602900 & -4.23904000 & -1.24460800 \\
\hline 1 & -3.66142400 & -3.15655200 & -2.61350400 \\
\hline 1 & -5.21922800 & -3.20796300 & -1.77633800 \\
\hline 6 & -5.32136500 & 0.40769900 & 1. 58009300 \\
\hline 1 & -5.09592600 & 1. 47937500 & 1. 57215300 \\
\hline 1 & -5.43232900 & 0.11102200 & 2. 62955900 \\
\hline 1 & -6.29006400 & 0.26459000 & 1. 09513600 \\
\hline 6 & -2.57045700 & 0.69011800 & 2. 34913100 \\
\hline 1 & -3.28670200 & 0.51622500 & 3. 15733200 \\
\hline 1 & -2.64355000 & 1. 75448400 & 2. 09191800 \\
\hline 1 & -1.58200300 & 0.49529200 & 2. 76518700 \\
\hline 7 & -0.26053700 & 1. 17699100 & 0.76666700 \\
\hline 7 & 0.61200600 & 1.59233000 & 1. 56905600 \\
\hline 7 & 1. 39657600 & 1. 89690600 & 2. 30616100 \\
\hline 6 & -1.01854900 & 2.21615000 & 0.10035200 \\
\hline 6 & -0.96856500 & 3.53288600 & 0.55717000 \\
\hline 6 & -1.79911100 & 1. 86041400 & -0.99634000 \\
\hline 6 & -1.71185700 & 4. 50611600 & -0.10164800 \\
\hline 6 & -2.53820600 & 2. 84957300 & -1.63864300 \\
\hline 6 & -2.49946000 & 4. 16905800 & -1.19897300 \\
\hline 1 & -0.37205800 & 3. 80728800 & 1. 42206500 \\
\hline 1 & -1.83759800 & 0.83509300 & -1.34106200 \\
\hline 1 & -1.67499900 & 5.53026900 & 0.25378100 \\
\hline 1 & -3.14667700 & 2. 57468900 & -2.49339400 \\
\hline 1 & -3.07839500 & 4. 93198300 & -1.70806600 \\
\hline \multicolumn{4}{|c|}{64} \\
\hline \multicolumn{4}{|c|}{ 1_2a } \\
\hline 6 & -1.99331100 & -0.27208400 & 0.15144600 \\
\hline 6 & -2.56595800 & -0.36544000 & 1. 60758400 \\
\hline 6 & -3.30632100 & 0.70150400 & 1. 91949200 \\
\hline 6 & -3.39043500 & 1. 72624700 & 0.77477500 \\
\hline 1 & -3.94250000 & 2. 62773500 & 1. 03837100 \\
\hline 6 & -3.93222600 & 1. 01307800 & -0.45705100 \\
\hline 6 & -3.21503600 & -0.06578900 & -0.76843000 \\
\hline 6 & 2. 15978400 & -0.40838200 & 0.13715600 \\
\hline 6 & 2. 61578100 & -1.16958200 & 1. 23285000 \\
\hline 6 & 3. 97819600 & -1.46710900 & 1. 36920600 \\
\hline 6 & 4. 86843400 & -0.97996300 & 0.41426200 \\
\hline 1 & 5. 92702900 & -1.21381500 & 0.51352900 \\
\hline 6 & 4. 44991800 & -0.19351700 & -0.65528000 \\
\hline 6 & 3. 08369700 & 0. 10299300 & -0.79520300 \\
\hline 5 & -0.71732900 & -1.17965100 & -0.35234400 \\
\hline
\end{tabular}

$\begin{array}{rrrr}5 & 0.60940500 & -0.20018900 & 0.00780800 \\ 35 & -0.78640800 & -3.17190700 & -0.03319900 \\ 35 & -0.44256600 & -0.84716100 & -2.44999000 \\ 6 & -3.60550500 & -1.09980700 & -1.78902400 \\ 1 & -3.36313700 & -0.78780700 & -2.80755700 \\ 1 & -4.68267100 & -1.28207900 & -1.73013100 \\ 1 & -3.08898500 & -2.04505900 & -1.60941900 \\ 6 & -5.17088900 & 1.53218200 & -1.11223000 \\ 1 & -6.01136400 & 1.54359900 & -0.40831200 \\ 1 & -5.45189500 & 0.92388100 & -1.97393200 \\ 1 & -5.02650900 & 2.56168300 & -1.45844500 \\ 6 & -4.04919600 & 1.01610200 & 3.17775900 \\ 1 & -3.68537500 & 1.95016000 & 3.62018100 \\ 1 & -3.95511800 & 0.22575300 & 3.92358700 \\ 1 & -5.11598700 & 1.15489000 & 2.96681600 \\ 6 & -2.33888400 & -1.56914700 & 2.46664900 \\ 1 & -2.74600100 & -1.40730500 & 3.46649500 \\ 1 & -1.27967500 & -1.81917200 & 2.55801000 \\ 1 & -2.82592200 & -2.44828900 & 2.03419600 \\ 6 & 1.65451100 & -1.64479100 & 2.29727700 \\ 1 & 2.05684300 & -1.46716600 & 3.30026900 \\ 1 & 1.44572600 & -2.71698900 & 2.21133200 \\ 1 & 0.69679700 & -1.12017000 & 2.23776900 \\ 6 & 4.47966200 & -2.29448300 & 2.52425300 \\ 1 & 3.94386000 & -3.24657800 & 2.60024900 \\ 1 & 4.34884800 & -1.77726700 & 3.48204300 \\ 1 & 5.54347800 & -2.51644600 & 2.41083200 \\ 6 & 5.45693000 & 0.32932300 & -1.64627400 \\ 1 & 5.45206200 & 1.42433200 & -1.68765300 \\ 1 & 5.24651200 & -0.02721000 & -2.66065900 \\ 1 & 6.46737700 & 0.00916700 & -1.38131000 \\ 6 & 2.66228100 & 0.97820700 & -1.94920800 \\ 1 & 2.96884600 & 0.54294000 & -2.90721000 \\ 1 & 3.13222700 & 1.96706800 & -1.87838600 \\ 1 & 1.58340000 & 1.11765800 & -1.99694500 \\ 7 & 0.02594300 & 1.13529600 & 0.17562000 \\ 7 & -1.33164700 & 1.12659900 & 0.24545900 \\ 7 & -1.99134600 & 2.13245600 & 0.50284300 \\ 6 & 0.68127200 & 2.40496600 & 0.28981800 \\ 6 & 0.44488300 & 3.39994200 & -0.65812600 \\ 6 & 1.61191000 & 2.58825000 & 1.30697300 \\ 6 & 1.14808900 & 4.59507500 & -0.57798900 \\ 6 & 2.31991700 & 3.78604300 & 1.37169400 \\ 6 & 2.08906200 & 4.78773000 & 0.43378900 \\ 1 & -0.27723500 & 3.22627400 & -1.45020400 \\ 1 & 1.78465300 & 1.79371600 & 2.02559900 \\ 1 & 0.97159100 & 5.37235700 & -1.31401400 \\ 1 & 3.05412500 & 3.93143800 & 2.15694300 \\ 1 & 2.64263600 & 5.71953800 & 0.48781800 \\ 64 & & & \end{array}$

\section{An2BBF2}

$\begin{array}{rrrr}6 & -1.35434700 & 0.26599300 & 0.16156300 \\ 6 & -2.57021000 & -0.40801900 & 0.44760200 \\ 6 & -2.61288600 & -1.58431300 & 1.26580500 \\ 1 & -1.69026200 & -1.99533500 & 1.66609800 \\ 6 & -3.78801200 & -2.22900400 & 1.53052900 \\ 1 & -3.78715300 & -3.11604500 & 2.15586600 \\ 6 & -5.01497100 & -1.75747500 & 0.98415400 \\ 1 & -5.93790800 & -2.28499300 & 1.20189800 \\ 6 & -5.01991800 & -0.65748100 & 0.17885600 \\ 1 & -5.94469600 & -0.29200200 & -0.25945800 \\ 6 & -3.80967800 & 0.04355100 & -0.11647200 \\ 6 & -3.80963200 & 1.15845800 & -0.95538700 \\ 1 & -4.75016500 & 1.50814200 & -1.37573300 \\ 6 & -2.63250100 & 1.83507100 & -1.26716100 \\ 6 & -2.65831700 & 2.97713100 & -2.13017200 \\ 1 & -3.61766800 & 3.29652700 & -2.52816800\end{array}$




\begin{tabular}{|c|c|c|c|}
\hline 6 & -1.51480100 & 3. 64854300 & -2.43764700 \\
\hline 1 & -1.54157100 & 4. 51472400 & -3.09085500 \\
\hline 6 & -0.27252300 & 3. 21283300 & -1.89264200 \\
\hline 1 & 0.63593400 & 3. 75386300 & -2.13796200 \\
\hline 6 & -0.20948700 & 2. 13276100 & -1.06206500 \\
\hline 1 & 0.75048400 & 1. 83476100 & -0.65948300 \\
\hline 6 & -1.38157000 & 1. 37979300 & -0.71716300 \\
\hline 6 & 1. 35206800 & -0.37707500 & 0.22198800 \\
\hline 6 & 2. 49316700 & 0.34819900 & 0.64477000 \\
\hline 6 & 2. 40923600 & 1. 38427000 & 1. 63243600 \\
\hline 1 & 1. 44780700 & 1. 62511100 & 2. 07828200 \\
\hline 6 & 3.50974800 & 2. 09732800 & 2. 01269000 \\
\hline 1 & 3. 41651100 & 2.87143000 & 2. 76766100 \\
\hline 6 & 4. 77957100 & 1.84423100 & 1. 41925000 \\
\hline 1 & 5. 64161400 & 2. 42648200 & 1. 72826000 \\
\hline 6 & 4. 90333300 & 0.88266000 & 0.46086600 \\
\hline 1 & 5. 86287200 & 0.68667000 & -0.01020500 \\
\hline 6 & 3. 77423600 & 0.11062500 & 0. 04269900 \\
\hline 6 & 3. 89163000 & -0.86355200 & -0.94971300 \\
\hline 1 & 4. 86370600 & -1.04764300 & -1.40230000 \\
\hline 6 & 2. 78972500 & -1.60434900 & -1.37433100 \\
\hline 6 & 2. 92563400 & -2.60951400 & -2.38450300 \\
\hline 1 & 3. 91061800 & -2.77758100 & -2.81171500 \\
\hline 6 & 1. 85127000 & -3.33798800 & -2.79631500 \\
\hline 1 & 1. 96387600 & -4.09928600 & -3.56146300 \\
\hline 6 & 0.56961800 & -3.10472400 & -2.21692800 \\
\hline 1 & -0.28022400 & -3.69353100 & -2.54724300 \\
\hline 6 & 0.40127500 & -2.15522200 & -1.25240100 \\
\hline 1 & -0.58337800 & -2.00315200 & -0.82260600 \\
\hline 6 & 1. 49811700 & -1.35382500 & -0.79171700 \\
\hline 5 & -0.11498900 & -0.30600900 & 2. 61867500 \\
\hline 5 & -0.03830900 & -0.13979100 & 0.91518700 \\
\hline 9 & -0.29858400 & 0.73749800 & 3. 42495000 \\
\hline 9 & 0.01052000 & -1.47667400 & 3. 24041300 \\
\hline 6 & -1.35434700 & 0.26599300 & 0.16156300 \\
\hline 6 & -2.57021000 & -0.40801900 & 0.44760200 \\
\hline 6 & -2.61288600 & -1.58431300 & 1. 26580500 \\
\hline 1 & -1.69026200 & -1.99533500 & 1. 66609800 \\
\hline 6 & -3.78801200 & -2.22900400 & 1. 53052900 \\
\hline 1 & -3.78715300 & -3.11604500 & 2. 15586600 \\
\hline 6 & -5.01497100 & -1.75747500 & 0.98415400 \\
\hline 1 & -5.93790800 & -2.28499300 & 1. 20189800 \\
\hline 6 & -5.01991800 & -0.65748100 & 0.17885600 \\
\hline 1 & -5.94469600 & -0.29200200 & -0.25945800 \\
\hline 6 & -3.80967800 & 0.04355100 & -0.11647200 \\
\hline 6 & -3.80963200 & 1. 15845800 & -0.95538700 \\
\hline 1 & -4.75016500 & 1. 50814200 & -1.37573300 \\
\hline 6 & -2.63250100 & 1. 83507100 & -1.26716100 \\
\hline 6 & -2.65831700 & 2. 97713100 & -2.13017200 \\
\hline 1 & -3.61766800 & 3. 29652700 & -2.52816800 \\
\hline 6 & -1.51480100 & 3. 64854300 & -2.43764700 \\
\hline 1 & -1.54157100 & 4. 51472400 & -3.09085500 \\
\hline 6 & -0.27252300 & 3. 21283300 & -1.89264200 \\
\hline 1 & 0.63593400 & 3. 75386300 & -2.13796200 \\
\hline 6 & -0.20948700 & 2. 13276100 & -1.06206500 \\
\hline 1 & 0.75048400 & 1. 83476100 & -0.65948300 \\
\hline 6 & -1.38157000 & 1. 37979300 & -0.71716300 \\
\hline 6 & 1. 35206800 & -0.37707500 & 0.22198800 \\
\hline 6 & 2. 49316700 & 0.34819900 & 0.64477000 \\
\hline 6 & 2. 40923600 & 1. 38427000 & 1. 63243600 \\
\hline 1 & 1. 44780700 & 1. 62511100 & 2. 07828200 \\
\hline 6 & 3. 50974800 & 2. 09732800 & 2. 01269000 \\
\hline 1 & 3. 41651100 & 2.87143000 & 2. 76766100 \\
\hline 6 & 4. 77957100 & 1. 84423100 & 1. 41925000 \\
\hline 1 & 5. 64161400 & 2. 42648200 & 1. 72826000 \\
\hline 6 & 4. 90333300 & 0.88266000 & 0.46086600 \\
\hline 1 & 5. 86287200 & 0.68667000 & -0.01020500 \\
\hline 6 & 3. 77423600 & 0.11062500 & 0.04269900 \\
\hline
\end{tabular}

$\begin{array}{rrr}6 & 3.89163000 & -0.86355200 \\ 1 & 4.86370600 & -1.04764300 \\ 6 & 2.78972500 & -1.60434900 \\ 6 & 2.92563400 & -2.60951400 \\ 1 & 3.91061800 & -2.77758100 \\ 6 & 1.85127000 & -3.33798800 \\ 1 & 1.96387600 & -4.09928600 \\ 6 & 0.56961800 & -3.10472400 \\ 1 & -0.28022400 & -3.69353100 \\ 6 & 0.40127500 & -2.15522200 \\ 1 & -0.58337800 & -2.00315200 \\ 6 & 1.49811700 & -1.35382500 \\ 5 & -0.11498900 & -0.30600900 \\ 5 & -0.03830900 & -0.13979100 \\ 9 & -0.29858400 & 0.73749800 \\ 9 & 0.01052000 & -1.47667400\end{array}$

$-0.94971300$

$-1.40230000$

$-1.37433100$

$-2.38450300$

$-2.81171500$

$-2.79631500$

$-3.56146300$

-2. 21692800

$-2.54724300$

$-1.25240100$

$-0.82260600$

$-0.79171700$

2. 61867500

0.91518700

3. 42495000

3. 24041300

21

$7 \quad 0.06121700$

$7-0.78349900$

1. 37604600

1. 45812300

1. 47197600

2. 47255200

2. 20078600

3. 77021300

3. 26590600

4. 82101500

4. 57071800

1. 18153400

3. 95606300

3. 06777800

5. 83557300

5. 39209500

$-0.82754800$

$-1.11975500$

$-1.00752900$

$-0.80656200$

$-1.24869400$

$-1.17325200$

$-1.62528400$

$-1.79853300$

$-1.79438600$

$-2.11500000$

$-1.57790200$

$-1.79069400$

$-2.09609900$

$-1.65619200$

$-1.95281800$

$-2.26717300$

$-1.85880300$

$-2.09190000$

$-1.48512000$

$-1.47018100$

$-1.17112000$

$-0.96174600$

$-1.20686600$

$-0.37056600$

0. 60976000

2. 00714500

2. 36390800

2. 93612000

3. 97334600

2. 56136700

3. 31487200

1. 25084200

0. 93193400

0. 24695000

$-1.09064800$

$-1.36100900$
0. 71470200

1. 63570600

2. 42138100

0. 70748300

0. 45691900

0. 91387900

0. 41375000

0. 88202200

0. 62914200

0. 30101900

1. 08425500

0. 21589200

1. 04352900

0. 59702800

$-0.14252700$

$-1.10085600$

$-2.51477100$

$-2.88125500$

$-3.42745600$

$-4.48789400$

$-3.00516600$

$-3.74375800$

$-1.67840200$

$-1.33636200$

$-0.69392800$

0. 65752900

0. 96936400

1. 61087700

2. 98937900

3. 27325000

3. 91882800

4. 96040300

3. 51371900

4. 24541100

2. 21203500

1. 91366800

1. 20074200

$-0.37223200$

$-0.41103200$

$-0.67074100$

$-0.89119600$

$-0.66415300$

$-0.88587900$

$-0.38872100$

$-0.37493000$

$-0.16578000$

0. 02584200

$-0.19011900$

$-0.00271300$

0. 17693800 


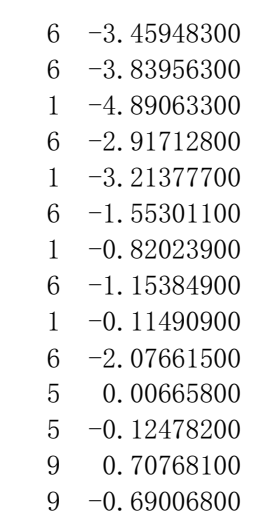

64

2_2

$\begin{array}{ll}7 & 1.99054700\end{array}$

$7 \quad 1.49880300$

$\begin{array}{ll}7 & 0.46807200\end{array}$

$\begin{array}{ll}6 & 3.41818400\end{array}$

64.08507500

64.15136400

$6 \quad 5.47507000$

6
6.53829000

$6 \quad 6.20666100$

13.51815600

13.63647300

$1 \quad 5.98081800$

$1 \quad 6.09904700$

$1 \quad 7.28963500$

$\begin{array}{ll}6 & -0.24568900\end{array}$

$\begin{array}{ll}6 & -1.00745400\end{array}$

$6-0.44852800$

$1 \quad 0.58093800$

$6-1.19558200$

$1-0.73822500$

$6-2.57717500$

$1-3.16151500$

$\begin{array}{ll}6 & -3.16077200\end{array}$

$1-4.21665700$

$6-2.39927000$

$\begin{array}{ll}6 & -2.99553600\end{array}$

$1-4.05564600$

$6-2.26120800$

$6-2.88760400$

$1-3.94689100$

$6-2.17783300$

$1-2.66067700$

$6-0.78986900$

$1-0.22747100$

$6-0.15813800$

$1 \quad 0.90327100$

$6-0.86419100$

$6-0.42985800$

$6-0.05289400$

$6 \quad 1.29840800$

$1 \quad 2.08579300$

$6 \quad 1.60819500$

$1 \quad 2.64251700$

$6 \quad 0.58872700$

$1 \quad 0.85694700$

$\begin{array}{ll}6 & -0.71498300\end{array}$

$1-1.50647900$

$6-1.08393400$

$\begin{array}{ll}6 & -2.41551800\end{array}$

$1-3.17680700$

$\begin{array}{ll}6 & -2.78989500\end{array}$

$\begin{array}{ll}6 & -4.15288500\end{array}$
$-2.08858900$

$-3.46325500$

$-3.68713600$

$-4.45914900$

$-5.49942300$

$-4.12712400$

$-4.92100800$

$-2.82699000$

$-2.61782800$

$-1.73170000$

1. 02785800

0. 11857200

2. 15551700

0. 68656200

0. 70651400

1. 27298100

0. 87723100

0.83228900

1. 29582400

0. 50941300

1. 36617000

0. 60358900

1. 01644000

1. 63588000

0. 19752500

1. 71857700

0. 34708400

1. 08052000

0. 50741900

$-0.58516700$

$-1.89453800$

$-2.08724200$

$-2.93903900$

$-3.91651100$

$-2.76828700$

$-3.61342400$

$-1.54637200$

$-1.39825300$

$-0.42328600$

0. 82561300

0.94574600

1. 92587900

3. 20066800

3. 28511900

4. 27789900

5. 24161600

4. 14174800

5. 00672900

2. 94007500

2. 86987400

1. 77303300

$-0.15859900$

$-1.50292300$

$-1.96321000$

$-1.26457300$

$-3.27354300$

$-3.59765600$

-4. 22339800

$-5.25947500$

$-3.82904800$

-4. 54160100

$-2.46520200$

$-2.05976600$

$-2.79230600$

$-0.73649800$

$-0.31408400$
$-0.07282400$

0. 05451400

0. 21722800

$-0.04044200$

0. 04952300

$-0.27852500$

$-0.38487600$

$-0.38625300$

$-0.59477400$

$-0.27152400$

$-1.96493400$

$-0.50448800$

$-2.12577400$

$-3.05686600$

$-0.39017300$

$-1.60941100$

$-2.15667100$

$-0.39095400$

0. 74582600

$-1.53588900$

0.76055800

$-1.52032200$

$-0.36995000$

1. 60827200

$-2.43867800$

1. 65360200

$-2.41333000$

$-0.36089700$

1. 05452100

1. 53910600

1. 70695700

1. 41531400

2. 17180900

2. 28794900

2. 46546100

2. 81557600

2. 29682600

2. 50822300

1. 84773100

1. 67098100

1. 88450300

1. 23110100

1. 06035300

1. 28922100

0. 62131100

0. 49384100

0. 33244600

$-0.00515000$

0. 48084100

0. 26585300

0.92450000

$-1.78206600$

$-1.59758900$

$-1.66992100$

$-1.92204300$

$-1.44211000$

$-1.49625000$

$-1.14455900$

$-0.96475300$

$-1.09800900$

$-0.88184600$

$-1.32210300$

$-1.22941200$

$-0.97158300$

$-1.46727300$

$-1.37957800$

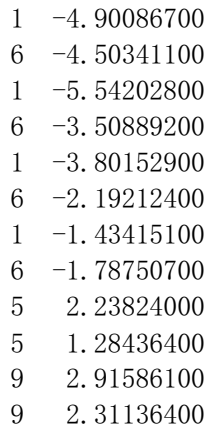

64

2_3

$7 \quad-0.30059900$

$\begin{array}{ll}7 & 0.64336200\end{array}$

$\begin{array}{ll}7 & 1.85691600\end{array}$

$6-1.46425500$

$\begin{array}{ll}6 & -2.04159000\end{array}$

$\begin{array}{ll}6 & -2.00113200\end{array}$

$6-3.16545700$

$\begin{array}{ll}6 & -3.13412500\end{array}$

$\begin{array}{ll}6 & -3.71641000\end{array}$

$1-1.60559200$

$1-1.53047700$

$1-3.61632400$

$1-3.55513500$

$1-4.59651300$

$\begin{array}{ll}6 & -1.53583800\end{array}$

$\begin{array}{ll}6 & -2.68865800\end{array}$

$6-2.76065600$

$1-1.89252200$

$\begin{array}{ll}6 & -3.88754300\end{array}$

$1-3.92004000$

$\begin{array}{ll}6 & -5.03090900\end{array}$

$1-5.92059500$

$6-5.00382000$

$1-5.86792600$

$\begin{array}{ll}6 & -3.83862800\end{array}$

$\begin{array}{ll}6 & -3.79745200\end{array}$

$1-4.67070600$

$\begin{array}{ll}6 & -2.65852700\end{array}$

$6-2.60916300$

$1-3.48584500$

$\begin{array}{ll}6 & -1.49379600\end{array}$

$1-1.46818400$

$6-0.35095600$

$1 \quad 0.53277400$

$\begin{array}{ll}1 & -0.35998300\end{array}$

$1 \quad 0.52152500$

$\begin{array}{ll}6 & -1.50894800\end{array}$

$\begin{array}{ll}6 & 2.51960700\end{array}$

$\begin{array}{ll}6 & 2.29285400\end{array}$

$6 \quad 1.34954200$

$1 \quad 0.81601000$

$6 \quad 1.13282200$

$1 \quad 0.41110800$

$6 \quad 1.84688900$

$1 \quad 1.64679100$

$6 \quad 2.79407500$

13.37115400

$6 \quad 3.06537800$

64.06083300

14.62959400

$6 \quad 4.35728900$

$6 \quad 5.40733700$

$1 \quad 5.97877300$

$6 \quad 5.68214000$

$-1.05123300$

0. 97761300

1. 28702000

1. 92727900

2. 94876200

1. 57146000

2. 30223400

0. 23038700

$-0.45089500$

0. 29190800

$-1.57864700$

0. 02469300

1. 24870000

2. 21467400

2. 01705200

1. 28514700

2. 50469000

0. 08640900

2. 51689500

0. 11017400

1. 32275300

3. 42833300

$-0.85188100$

3. 46556400

$-0.82467700$

1. 33808800

$-0.19223000$

0. 49331200

1. 92507700

2. 49636000

2. 57602200

3. 66091200

1. 84180300

2. 37751300

0. 47958600

$-0.08935800$

$-0.24021200$

$-1.63480900$

-2. 18912900

$-2.33257900$

$-3.76250400$

$-4.30405900$

$-4.42948800$

$-5.51453900$

$-3.70686400$

-4. 24776400 


\begin{tabular}{|c|c|c|c|}
\hline 1 & 6. 48073000 & 1. 54802900 & -2.65879400 \\
\hline 6 & 4. 91862800 & 2. 51010600 & -1.48900500 \\
\hline 1 & 5. 15415900 & 3. 50102800 & -1.86384100 \\
\hline 6 & 3. 89923800 & 2. 34873100 & -0.59718200 \\
\hline 1 & 3. 31429700 & 3. 19841300 & -0.26302400 \\
\hline 6 & 3. 58680800 & 1. 05169000 & -0.08221200 \\
\hline 5 & 1. 01942000 & 0.74395400 & -1.48847700 \\
\hline 5 & -0.26458200 & 0.60502300 & -0.38128300 \\
\hline 9 & 1. 34754400 & 1.91198900 & -2.03741500 \\
\hline 9 & 1. 64217100 & -0.32369300 & -1.97967300 \\
\hline \multicolumn{4}{|l|}{64} \\
\hline \multicolumn{4}{|c|}{ 2_4 } \\
\hline 7 & -0.38319200 & 0.44073700 & -0.79500900 \\
\hline 7 & 0.96793200 & 0.52477700 & -0.87020900 \\
\hline 7 & 1. 54372800 & -0.20252400 & -0.03333600 \\
\hline 6 & -1.06285200 & 1. 24685300 & -1.76552200 \\
\hline 6 & -2.19727700 & 0.73499000 & -2.38972100 \\
\hline 6 & -0.60942400 & 2. 53378700 & -2.04910900 \\
\hline 6 & -2.89341800 & 1. 52952000 & -3.29417000 \\
\hline 6 & -1.30441700 & 3. 31240100 & -2.96845100 \\
\hline 6 & -2.45002500 & 2. 81652300 & -3.58612100 \\
\hline 1 & -2.52774800 & -0.27296900 & -2.16237200 \\
\hline 1 & 0.27773400 & 2. 91473500 & -1.55267000 \\
\hline 1 & -3.78168000 & 1. 13425900 & -3.77587100 \\
\hline 1 & -0.95427500 & 4. 31465500 & -3.19326100 \\
\hline 1 & -2.99557400 & 3. 43149400 & -4.29430800 \\
\hline 6 & -2.44826400 & -0.50027100 & 0.54036500 \\
\hline 6 & -3.07825400 & -1.76270900 & 0.47415100 \\
\hline 6 & -2.34121400 & -2.96339800 & 0.20443000 \\
\hline 1 & -1.25975500 & -2.90975900 & 0.11074500 \\
\hline 6 & -2.97013200 & -4.16987500 & 0.10681200 \\
\hline 1 & -2.39136900 & -5.06792700 & -0.08484700 \\
\hline 6 & -4.38426400 & -4.26942700 & 0.26301700 \\
\hline 1 & -4.86512900 & -5.23853500 & 0.17497400 \\
\hline 6 & -5.12266200 & -3.15594100 & 0.53014200 \\
\hline 1 & -6.19987400 & -3.21935300 & 0.65937600 \\
\hline 6 & -4.50046200 & -1.87220300 & 0.64727900 \\
\hline 6 & -5.24596900 & -0.72570200 & 0.92276000 \\
\hline 1 & -6.32275500 & -0.80974400 & 1. 05422600 \\
\hline 6 & -4.63948700 & 0.52620800 & 1. 03727900 \\
\hline 6 & -5.40401800 & 1. 69757600 & 1. 34082100 \\
\hline 1 & -6.47554400 & 1. 58843000 & 1. 48602700 \\
\hline 6 & -4.80523300 & 2. 91669000 & 1. 44893200 \\
\hline 1 & -5.39350000 & 3. 79882400 & 1. 68058300 \\
\hline 6 & -3.39602800 & 3. 03859500 & 1. 27057100 \\
\hline 1 & -2.92751800 & 4. 01221800 & 1. 37301500 \\
\hline 6 & -2.63626900 & 1.94312800 & 0.98429500 \\
\hline 1 & -1.56055300 & 2. 05189600 & 0.87594000 \\
\hline 6 & -3.22168400 & 0.64258600 & 0.84358500 \\
\hline 6 & 2. 96948600 & -0.08540200 & 0.04668600 \\
\hline 6 & 3. 75652400 & -1.15868400 & -0.38991700 \\
\hline 6 & 3. 20355100 & -2.34615300 & -0.96462900 \\
\hline 1 & 2. 12671600 & -2.44505300 & -1.03717400 \\
\hline 6 & 4. 02249800 & -3.35170300 & -1.38617200 \\
\hline 1 & 3. 59340400 & -4.25202500 & -1.81380800 \\
\hline 6 & 5. 43987100 & -3.24088800 & -1.26994500 \\
\hline 1 & 6. 06847600 & -4.05633400 & -1.61274700 \\
\hline 6 & 5. 99999600 & -2.12191500 & -0.73064500 \\
\hline 1 & 7. 07809800 & -2.02617700 & -0.63723400 \\
\hline 6 & 5. 18220100 & -1.04128100 & -0.27336200 \\
\hline 6 & 5. 73709600 & 0.11686400 & 0.27321900 \\
\hline 1 & 6. 81844200 & 0.19896400 & 0.35412300 \\
\hline 6 & 4. 94032800 & 1. 17006100 & 0.72359000 \\
\hline 6 & 5. 51210200 & 2. 35122700 & 1. 29369600 \\
\hline 1 & 6. 59455100 & 2. 41775800 & 1. 35754800 \\
\hline 6 & 4. 72060000 & 3. 36132700 & 1. 75072200 \\
\hline 1 & 5. 16352100 & 4. 25184400 & 2. 18445500 \\
\hline 6 & 3. 30056800 & 3. 24845800 & 1. 67788100 \\
\hline
\end{tabular}

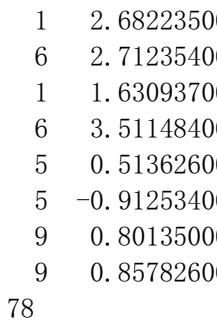

2_5

$\begin{array}{ll}7 & -0.14976300\end{array}$

$7-1.44711400$

$\begin{array}{ll}7 & -1.83200500\end{array}$

$\begin{array}{ll}6 & 0.28575100\end{array}$

$6-0.22211500$

$6 \quad 1.22077100$

$\begin{array}{ll}6 & 0.21256800\end{array}$

$6 \quad 1.65880100$

6 1. 15854200

$1-0.95238900$

$1 \quad 1.59609100$

$1-0.18547200$

$1 \quad 2.38889200$

$1 \quad 1.50084900$

$6 \quad 2.17795100$

63.15688100

$6 \quad 2.83197300$

$1 \quad 1.80341900$

63.78694500

13.49363100

65.16445300

15.91010600

$6 \quad 5.53150800$

16.57736400

$6 \quad 4.55691800$

$6 \quad 4.96119100$

16.02067300

$6 \quad 4.03636900$

64.47117000

15.53837600

$6 \quad 3.57464600$

13.91130200

62.18385900

$1 \quad 1.47092600$

$6 \quad 1.73268900$

10.67126600

$6 \quad 2.63204100$

$\begin{array}{ll}6 & -3.24468400\end{array}$

$\begin{array}{ll}6 & -3.73019900\end{array}$

$\begin{array}{ll}6 & -2.87012800\end{array}$

$1-1.79692200$

$\begin{array}{ll}6 & -3.39245400\end{array}$

$1-2.72971500$

$6-4.80336100$

$1-5.19436400$

$\begin{array}{ll}6 & -5.65188000\end{array}$

$1-6.72785900$

$6-5.14860700$

$\begin{array}{ll}6 & -6.00202600\end{array}$

$1-7.07682800$

$\begin{array}{ll}6 & -5.50981000\end{array}$

$\begin{array}{ll}6 & -6.38875000\end{array}$

$1-7.45874000$

$6-5.89552800$

$1-6.56896300$

$\begin{array}{ll}6 & -4.48663600\end{array}$

$1-4.10766700$

$\begin{array}{ll}6 & -3.61257900\end{array}$
4. 04856400

2. 14503400

2. 06343800

1. 07377700

$-1.02135200$

$-0.39556700$

$-2.36842400$

$-0.52333800$

$-1.24728600$

$-1.01945000$

$-0.07641700$

$-2.35164400$

$-2.52260600$

$-3.24459300$

$-3.59532700$

$-4.30527800$

$-4.48497200$

$-1.81892900$

$-3.10726200$

$-3.72888700$

$-4.99974600$

$-5.31682200$

$-0.52808500$

$-0.45351100$

$-0.43261700$

$-0.55696000$

$-0.31941000$

$-0.32861900$

$-0.20517000$

$-0.10535700$

$-0.24114300$

$-0.17358000$

$-0.37792600$

$-0.43456900$

$-0.36584300$

$-0.59075000$

$-0.68761300$

$-0.63926600$

$-0.84211700$

$-0.91607400$

$-0.91621700$

$-1.05520900$

$-0.82052300$

$-0.90453400$

$-0.64161200$

0. 12762600

1. 40464400

2. 49670200

2. 35563900

3. 71045700

4. 53512800

3. 91727900

4. 89073300

2. 90427700

3. 05389300

1. 61669800

0. 56738300

0. 73247600

$-0.68339800$

$-1.74448200$

$-1.55740600$

$-2.95000000$

$-3.74691800$

$-3.16725400$

$-4.12086600$

$-2.19136800$
2. 07192100

1. 13427200

1. 11411500

0. 62114000

1. 03561100

0. 28134200

0. 90153600

2. 27236100

$-0.60936600$

$-0.69124500$

0. 06643400

$-1.40926100$

$-2.69774800$

$-0.88992300$

$-3.46794000$

$-1.67682700$

$-2.96375000$

$-3.08428700$

0. 11830900

$-4.46866500$

$-1.27430800$

$-3.57056300$

0. 62916000

$-0.39935000$

$-1.79881500$

$-2.11227200$

$-2.76736100$

$-3.81293400$

$-2.42589500$

$-3.20804300$

$-1.11587800$

$-0.82791600$

$-0.07635400$

1. 25558100

1. 49383400

2. 28384400

3. 64416500

3. 84411200

4. 65713400

5. 68643100

4. 35864800

5. 16537800

3. 07403400

2. 88655300

1. 96970700

0. 12728700

$-0.19300300$

$-0.53308300$

$-0.46890900$

$-0.86978600$

$-1.11189300$

$-0.89723200$

$-1.17527600$

$-0.56501700$

$-0.57071100$

$-0.19664700$

0. 14670400

0. 13367600

0. 52069400

0. 90800000

0. 88655000

1. 30516400

1. 60369800

1. 35272800

1. 70576400

0. 97589900 


\begin{tabular}{|c|c|}
\hline 1 & -2.54481400 \\
\hline 6 & -4.09292300 \\
\hline 5 & -0.72743000 \\
\hline 5 & 0.61547600 \\
\hline 9 & -0.81656400 \\
\hline 9 & -1.24300900 \\
\hline 7 & 0.66460100 \\
\hline 7 & 0.11029900 \\
\hline 7 & -0.45206500 \\
\hline 6 & 1.57676900 \\
\hline 6 & 1. 85101200 \\
\hline 6 & 2. 20072600 \\
\hline 6 & 2. 77654600 \\
\hline 6 & 3. 11267300 \\
\hline 6 & 3. 40518200 \\
\hline 1 & 1. 33743500 \\
\hline 1 & 1. 99438500 \\
\hline 1 & 3. 00130500 \\
\hline 1 & 3. 60339100 \\
\hline 1 & 4. 12505300 \\
\hline \multicolumn{2}{|l|}{76} \\
\hline \multicolumn{2}{|l|}{ 2_P } \\
\hline 7 & -4.54573000 \\
\hline 7 & -5.84886600 \\
\hline 7 & -6.40806000 \\
\hline 6 & -4.02496500 \\
\hline 6 & -4.47447500 \\
\hline 6 & -3.06087100 \\
\hline 6 & -3.94800000 \\
\hline 6 & -2.52987600 \\
\hline 6 & -2.97272200 \\
\hline 1 & -5.22771500 \\
\hline 1 & -2.73259900 \\
\hline 1 & -4.29511800 \\
\hline 1 & -1.77310700 \\
\hline 1 & -2.55859100 \\
\hline 6 & -2.12812800 \\
\hline 6 & -1.72280100 \\
\hline 6 & -2.66453200 \\
\hline 1 & -3.72178700 \\
\hline 6 & -2.25981500 \\
\hline 1 & -2.99069200 \\
\hline 6 & -0.87812000 \\
\hline 1 & -0.57476300 \\
\hline 6 & 0.05303400 \\
\hline 1 & 1. 10928400 \\
\hline 6 & -0.33260300 \\
\hline 6 & 0.61025000 \\
\hline 1 & 1.66388800 \\
\hline 6 & 0.23110000 \\
\hline 6 & 1. 19725600 \\
\hline 1 & 2. 24546400 \\
\hline 6 & 0.81337300 \\
\hline 1 & 1. 55406400 \\
\hline 6 & -0.56628200 \\
\hline 1 & -0.85676900 \\
\hline 6 & -1.51728200 \\
\hline 1 & -2.56527500 \\
\hline 6 & -1.15969300 \\
\hline 6 & -7.82215800 \\
\hline 6 & -8.26226200 \\
\hline 6 & -7.37158700 \\
\hline 1 & -6.30843900 \\
\hline 6 & -7.85610600 \\
\hline 1 & -7.17371100 \\
\hline 6 & -9.25355100 \\
\hline 1 & -9.61304800 \\
\hline 6 & -10.12963300 \\
\hline
\end{tabular}

$\begin{array}{rr}-2.36267300 & 1.04739100 \\ -0.91885000 & 0.52693700 \\ 0.28437100 & 1.29028100 \\ -0.31120100 & 0.34599200 \\ 1.63441400 & 1.58201000 \\ -0.47797500 & 2.33901000 \\ 1.26461800 & -0.75380300 \\ 1.19327100 & -1.86267000 \\ 0.99583100 & -2.81740000 \\ 2.35878600 & -0.54904200 \\ 2.72403200 & 0.76515000 \\ 2.97875400 & -1.62869800 \\ 3.73745000 & 0.99103900 \\ 3.99913600 & -1.38232700 \\ 4.37741000 & -0.07481900 \\ 2.22947700 & 1.58082900 \\ 2.65840300 & -2.64626000 \\ 4.02882100 & 2.01158500 \\ 4.48782900 & -2.21742700 \\ 5.16718200 & 0.11248100\end{array}$

0. 32035700

0. 18496200

0.76897000

$-0.25786600$

$-1.49983400$

0. 45070200

$-2.03440100$

$-0.10122700$

$-1.34139900$

$-2.03286000$

1. 42259000

$-3.00139700$

0.44521000

$-1.76735100$

0. 34280100

$-1.00462800$

$-2.07216500$

$-1.83305400$

$-3.37373600$

$-4.16515100$

$-3.70737600$

$-4.74863700$

$-2.72441100$

$-2.96652100$

$-1.34746100$

$-0.32902900$

$-0.58657000$

1. 01352300

2. 05810000

1. 78267700

3. 36471500

4. 14949100

3. 71137000

4. 75671400

2. 74471100

3. 02330900

1. 35641800

0. 51695800

$-0.34190000$

$-0.96694600$

$-0.75326400$

$-1.80374300$

$-2.26575400$

$-2.07532300$

$-2.74557400$

$-1.49490700$
1. 61439900

1. 50544500

0. 53347600

2. 82613100

3. 26584600

3. 53606900

4. 43702100

4. 69724900

5. 15006700

2. 69505200

3. 18037400

4. 78587100

5. 25016400

6. 05807900

0. 56174400

0. 51315800

0. 34106700

0. 23925400

0. 29069000

0. 15808000

0. 40820200

0. 36680300

0. 56503100

0. 64803800

0.61962300

0. 76838400

0. 80650800

0. 95921100

1. 03923600

1. 00393900

1. 11999600

0. 89667100

0. 92920100

0. 74751800

0. 65738300

0. 69560900

0. 42888800

$-0.58444500$

$-1.51386800$

$-1.45554600$

$-2.47499100$

$-3.18114500$

$-2.57269700$

-3. 34668700

$-1.70524100$
0. 85333600

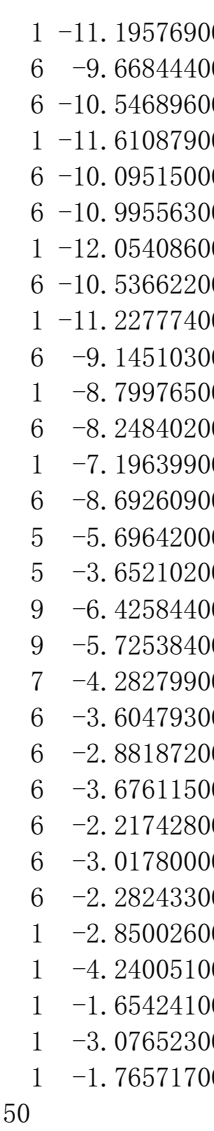

2_2a

7 2. 12579300

$7 \quad 1.96177800$

$\begin{array}{ll}7 & 2.87689800\end{array}$

$6 \quad 3.42596600$

$6 \quad 3.66914800$

$6 \quad 4.39763800$

$6 \quad 4.89046500$

$6 \quad 5.61492100$

$6 \quad 5.86636300$

$1 \quad 2.90056000$

14.20065400

15.07841800

$1 \quad 6.36933400$

$1 \quad 6.81921300$

$6 \quad 0.45305800$

$6 \quad 0.55674800$

$\begin{array}{ll}6 & -0.15186700\end{array}$

$1-0.87693000$

$\begin{array}{ll}6 & 0.07876400\end{array}$

$1-0.47842700$

$6 \quad 1.02367800$

$1 \quad 1.19419100$

61.77245500

12.53844600

$6 \quad 1.53153600$

$6 \quad 2.30744400$

13.14194200

$6 \quad 1.33275900$

$6 \quad 1.37895900$

12.16079800

$6 \quad 0.40085900$

10.41604200

$6-0.58938300$

$1-1.34301600$

$\begin{array}{ll}6 & -0.62317600\end{array}$

$\begin{array}{ll}1 & -1.39596600\end{array}$
$-1.69243400$

$-0.60813600$

$-0.00643500$

$-0.21905300$

0. 86324600

1. 48368900

1. 25302000

2. 34256600

2. 80801800

2. 64767600

3. 35239400

2. 07389600

2. 33271700

1. 15120700

1. 86980100

0. 71895700

1. 95511400

3. 05034800

1. 34350000

1. 67022300

0. 70161200

2. 97381400

1. 04051300

3. 30271700

2. 34057200

$-0.31520100$

3. 72126600

0. 28093100

4. 31798900

2. 60150900

0. 91837800

$-0.34562800$

$-1.17281700$

1. 48473200

2. 37566000

1. 25814000

3. 03442900

1. 92494800

2. 81282400

2. 53179100

0. 57275100

3. 72384500

1. 75185400

3. 32905500

$-0.80947300$

$-1.82401300$

$-1.79274200$

$-1.00663400$

$-2.79542700$

$-2.79182000$

$-3.78991600$

$-4.56137200$

$-3.78852500$

$-4.53869700$

$-2.79904900$

$-2.55001500$

$-3.23415400$

$-2.51408100$

$-3.27634700$

$-4.01649000$

$-3.07431500$

$-3.66757200$

$-2.11278400$

$-1.96082400$

$-1.33676700$

$-0.58761800$
$-1.77266100$

$-0.68190600$

0. 22116700

0. 14690000

1. 21460400

2. 13730400

2. 05615900

3. 09003000

3. 78523900

3. 17433600

3. 92384100

2. 32270500

2. 37463300

1. 32250400

$-0.40455400$

0.47186300

$-1.56013000$

0. 33177200

$-0.59960900$

$-1.80941600$

$-2.50694800$

$-2.30720500$

$-3.68115400$

$-3.48696600$

$-4.17547000$

$-2.12650500$

$-1.75677000$

$-4.21420900$

$-3.86648200$

$-5.09336500$

$-0.09492700$

0. 17927800

0. 39213100

$-0.16060700$

$-1.20891800$

0. 81717300

$-1.27768900$

0. 73422700

-0. 30907100

$-1.95709600$

1. 63258600

$-2.09440200$

1. 49495800

$-0.36705000$

0. 15515400

$-0.98571000$

$-2.17813600$

$-2.36773800$

$-3.12078900$

$-4.05150200$

$-2.88018400$

$-3.62412800$

$-1.70145700$

$-1.52921300$

$-0.76033600$

0. 52029600

0. 66770700

1. 67367500

2. 83063500

2. 97192500

3. 80599900

4. 71454800

3. 62310900

4. 38862000

2. 46300200

2. 32223200 


\begin{tabular}{|c|c|c|c|}
\hline 6 & 0.33977000 & -1.55075100 & 1. 48402000 \\
\hline 6 & -1.96795500 & 0.70075000 & 0.07266900 \\
\hline 6 & -2.47535000 & 2. 01736900 & 0.31349200 \\
\hline 6 & -1.66149700 & 3. 05975900 & 0.85654700 \\
\hline 1 & -0.63238000 & 2. 84770600 & 1. 10781800 \\
\hline 6 & -2.15745800 & 4. 31159500 & 1. 08741700 \\
\hline 1 & -1.50659700 & 5. 07372500 & 1. 50435800 \\
\hline 6 & -3.51215100 & 4. 63226100 & 0.79518100 \\
\hline 1 & -3.88216200 & 5. 63621200 & 0.97694600 \\
\hline 6 & -4.33753100 & 3. 66591500 & 0.30434400 \\
\hline 1 & -5.38260300 & 3. 87454500 & 0. 09190600 \\
\hline 6 & -3.85392400 & 2. 34064600 & 0.07353100 \\
\hline 6 & -4.71573800 & 1. 34396000 & -0.37899200 \\
\hline 1 & -5.75079500 & 1. 59504700 & -0.60302400 \\
\hline 6 & -4.29547900 & 0.02285100 & -0.50762000 \\
\hline 6 & -5.23166200 & -0.98645500 & -0.89852900 \\
\hline 1 & -6.24725800 & -0.67751000 & -1.13114800 \\
\hline 6 & -4.86696600 & -2.29652400 & -0.95956800 \\
\hline 1 & -5.58164600 & -3.05981100 & -1.24974700 \\
\hline 6 & -3.53761600 & -2.66237300 & -0.61011100 \\
\hline 1 & -3.25333100 & -3.71025900 & -0.60698100 \\
\hline 6 & -2.61285800 & -1.71746200 & -0.26687600 \\
\hline 1 & -1.62821800 & -2.05102200 & 0.01946000 \\
\hline 6 & -2.92371300 & -0.32016400 & -0.22942800 \\
\hline 5 & 0.78104100 & 1. 64108100 & -0.64933200 \\
\hline 5 & -0.41411400 & 0.53193300 & -0.04508000 \\
\hline 9 & 0.92021100 & 2. 97774300 & -0.30775300 \\
\hline 9 & 0.79052000 & 1. 50344800 & -2.05147600 \\
\hline \multicolumn{4}{|l|}{78} \\
\hline \multicolumn{4}{|c|}{ 2_2a } \\
\hline 7 & 2. 12579300 & 0.91837800 & -0.09492700 \\
\hline 7 & 1.96177800 & -0.34562800 & 0.17927800 \\
\hline 7 & 2. 87689800 & -1.17281700 & 0. 39213100 \\
\hline 6 & 3. 42596600 & 1. 48473200 & -0.16060700 \\
\hline 6 & 3. 66914800 & 2. 37566000 & -1.20891800 \\
\hline 6 & 4. 39763800 & 1. 25814000 & 0.81717300 \\
\hline 6 & 4. 89046500 & 3. 03442900 & -1.27768900 \\
\hline 6 & 5. 61492100 & 1. 92494800 & 0.73422700 \\
\hline 6 & 5.86636300 & 2.81282400 & -0.30907100 \\
\hline 1 & 2.90056000 & 2. 53179100 & -1.95709600 \\
\hline 1 & 4. 20065400 & 0.57275100 & 1. 63258600 \\
\hline 1 & 5. 07841800 & 3. 72384500 & -2.09440200 \\
\hline 1 & 6. 36933400 & 1. 75185400 & 1. 49495800 \\
\hline 1 & 6. 81921300 & 3. 32905500 & -0.36705000 \\
\hline 6 & 0.45305800 & -0.80947300 & 0.15515400 \\
\hline 6 & 0.55674800 & -1.82401300 & -0.98571000 \\
\hline 6 & -0.15186700 & -1.79274200 & -2.17813600 \\
\hline 1 & -0.87693000 & -1.00663400 & -2.36773800 \\
\hline 6 & 0.07876400 & -2.79542700 & -3.12078900 \\
\hline 1 & -0.47842700 & -2.79182000 & -4.05150200 \\
\hline 6 & 1. 02367800 & -3.78991600 & -2.88018400 \\
\hline 1 & 1. 19419100 & -4.56137200 & -3.62412800 \\
\hline 6 & 1.77245500 & -3.78852500 & -1.70145700 \\
\hline 1 & 2. 53844600 & -4.53869700 & -1.52921300 \\
\hline 6 & 1. 53153600 & -2.79904900 & -0.76033600 \\
\hline 6 & 2. 30744400 & -2.55001500 & 0.52029600 \\
\hline 1 & 3. 14194200 & -3.23415400 & 0.66770700 \\
\hline 6 & 1. 33275900 & -2.51408100 & 1. 67367500 \\
\hline 6 & 1. 37895900 & -3.27634700 & 2. 83063500 \\
\hline 1 & 2. 16079800 & -4.01649000 & 2. 97192500 \\
\hline 6 & 0.40085900 & -3.07431500 & 3. 80599900 \\
\hline 1 & 0.41604200 & -3.66757200 & 4. 71454800 \\
\hline 6 & -0.58938300 & -2.11278400 & 3. 62310900 \\
\hline 1 & -1.34301600 & -1.96082400 & 4. 38862000 \\
\hline 6 & -0.62317600 & -1.33676700 & 2. 46300200 \\
\hline 1 & -1.39596600 & -0.58761800 & 2. 32223200 \\
\hline 6 & 0.33977000 & -1.55075100 & 1. 48402000 \\
\hline 6 & -1.96795500 & 0.70075000 & 0.07266900 \\
\hline
\end{tabular}

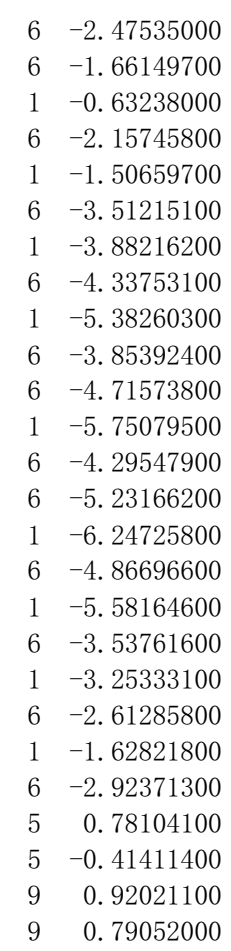

78

2_1'

7 2. 10668100

$7 \quad 2.15798000$

$7 \quad 2.12981000$

63.37976500

$6 \quad 4.46854000$

63.46578600

$6 \quad 5.67525500$

$6 \quad 4.67782800$

$6 \quad 5.78195200$

14.36835000

$1 \quad 2.59919200$

16.52877300

14.75748500

16.72379800

$6 \quad 0.11614300$

$6 \quad 1.30890800$

$6 \quad 1.87941200$

$1 \quad 1.39737600$

$6 \quad 3.02142500$

13.43374400

$6 \quad 3.68507200$

14.58967200

63.17951900

13.66828600

$6 \quad 1.98444100$

61.46919800

$1 \quad 1.99214700$

$6 \quad 0.29037800$

$\begin{array}{ll}6 & -0.22765200\end{array}$

$1 \quad 0.32098700$

$\begin{array}{ll}6 & -1.37468300\end{array}$

$1-1.76122900$

$\begin{array}{ll}6 & -2.07351000\end{array}$

$1-2.99115600$

$\begin{array}{ll}6 & -1.60719800\end{array}$

$1-2.16523600$

$\begin{array}{ll}6 & -0.40709000\end{array}$

$\begin{array}{ll}6 & -2.01980400\end{array}$

$\begin{array}{ll}6 & -2.94487900\end{array}$

$\begin{array}{ll}6 & -2.60276500\end{array}$
2. 01736900

3. 05975900

2. 84770600

4. 31159500

5. 07372500

4. 63226100

5. 63621200

3. 66591500

3. 87454500

2. 34064600

1. 34396000

1. 59504700

0. 02285100

$-0.98645500$

$-0.67751000$

$-2.29652400$

$-3.05981100$

$-2.66237300$

$-3.71025900$

$-1.71746200$

$-2.05102200$

$-0.32016400$

1. 64108100

0. 53193300

2. 97774300

1. 50344800

0. 31349200

0. 85654700

1. 10781800

1. 08741700

1. 50435800

0. 79518100

0.97694600

0. 30434400

0. 09190600

0. 07353100

$-0.37899200$

$-0.60302400$

$-0.50762000$

$-0.89852900$

$-1.13114800$

$-0.95956800$

$-1.24974700$

$-0.61011100$

$-0.60698100$

$-0.26687600$

0. 01946000

$-0.22942800$

$-0.64933200$

$-0.04508000$

$-0.30775300$

$-2.05147600$

$-1.07431400$

$-1.11364200$

$-0.03440000$

0.90493000

$-1.62536900$

$-0.79456700$

$-3.00678200$

$-1.36708300$

$-3.56100500$

$-2.74825700$

0. 28482000

$-3.62474000$

$-0.72773400$

$-4.63721400$

$-3.18993000$

1. 15331200

1. 35369300

0. 31863000

$-0.65452600$

0. 52285400

$-0.28564600$

1. 78314900

1. 92935400

2. 79818300

3. 76851200

2. 62070600

3. 65831000

4. 61192900

3. 50195200

4. 57864900

5. 51665400

4. 43215000

5. 25403800

3. 19070200

3. 08099700

2. 14124400

1. 21364300

2. 24255600

$-0.58827700$

$-1.10026700$

$-1.27221900$
$-1.80229000$

$-2.41419000$

$-0.72846900$

$-0.47506400$

$-0.58763100$

$-0.08875500$

$-0.18924300$

0. 05688200

$-0.55844100$

$-0.79005700$

0.11143000

$-0.07693500$

0. 01618700

0. 75956100

1. 57384500

1. 61491100

2. 29462700

2. 89017900

2. 26439100

2. 84637200

1. 50733900

1. 47679400

0. 74004000

$-0.03637500$

$-0.06685300$

$-0.76235000$

$-1.55057900$

$-1.55848800$

$-2.26944200$

$-2.86373200$

$-2.24171100$

$-2.81108600$

$-1.50545500$

$-1.50032000$

$-0.72584200$

0. 29626100

$-0.64705700$

$-2.02946600$
0. 36512100 


$\begin{array}{rr}1 & -1.59455700 \\ 6 & -3.51200100 \\ 1 & -3.22130400 \\ 6 & -4.84406400 \\ 1 & -5.55400300 \\ 6 & -5.21976800 \\ 1 & -6.23381600 \\ 6 & -4.29449400 \\ 6 & -4.68438200 \\ 1 & -5.70326600 \\ 6 & -3.79452300 \\ 6 & -4.20167700 \\ 1 & -5.22167900 \\ 6 & -3.33597500 \\ 1 & -3.65367300 \\ 6 & -2.00220300 \\ 1 & -1.31646300 \\ 6 & -1.57955300 \\ 1 & -0.55919800 \\ 6 & -2.45086900 \\ 5 & 0.43267600 \\ 5 & -0.51910600 \\ 9 & 0.08190600 \\ 9 & 0.81003000 \\ 64 & \end{array}$

\begin{abstract}
$-1.03832000$
$-1.72533100$

$-1.85031700$

$-2.03867800$

$-2.39892700$

$-1.88147400$

$-2.10934100$

$-1.40512600$

$-1.22536400$

$-1.46869200$

$-0.73713500$

$-0.56206600$

$-0.82612700$

$-0.08377700$

0. 04327800

0. 25134800

0. 63405300

0. 09569900

0. 35857100

$-0.40032900$

$-1.66927700$

$-0.29056200$

$-2.37797800$

$-2.48633900$
\end{abstract}

64

\section{An2B2Br2}

$6 \quad 1.93223300$

$6 \quad 1.88056100$

$6 \quad 0.68916900$

$1-0.20625500$

$6 \quad 0.65315800$

$1-0.26348200$

$6 \quad 1.81216100$

$1 \quad 1.76473300$

$6 \quad 2.96941300$

13.85951200

63.04464800

64.21970300

$1 \quad 5.10198100$

$6 \quad 4.28761400$

$6 \quad 5.49117700$

$1 \quad 6.36192800$

$6 \quad 5.54411900$

16.46158900

$6 \quad 4.39215000$

14.44732100

63.22789200

$1 \quad 2.35996600$

$6 \quad 3.12561100$

$6-1.93221800$

$\begin{array}{ll}6 & -3.12523900\end{array}$

$6-3.22681600$

$1 \quad-2.35858200$

$\begin{array}{ll}6 & -4.39078200\end{array}$

$1-4.44543000$

$6-5.54311700$

$1-6.46034400$

$6-5.49080800$

$1-6.36182300$

$6-4.28759500$

$6-4.22039400$

$1-5.10296500$

$\begin{array}{ll}6 & -3.04571900\end{array}$

$\begin{array}{ll}6 & -2.97119200\end{array}$

$1-3.86160700$

$\begin{array}{ll}6 & -1.81425400\end{array}$

$1-1.76735700$

$-1.81254900$
-2. 35579500

$-2.94023800$

$-3.97856100$

$-2.54266400$

$-3.28045600$

$-1.24244100$

$-0.92441400$

$-0.26018900$

1. 06629000

1. 36014100

2. 02135100

3. 38236700

3. 64887600

4. 31860100

5. 34870900

3. 94479400

4. 69407600

2. 65738700

2. 40511900

1. 63222500

$-0.59586200$

-0. 10450300

$-1.71327700$

0. 44228100

0. 00754700

1. 32773200

1. 87432900

1. 25771700

3. 15661600

3. 55390600

3. 98504600

5. 00161400

3. 50244900

4. 12341300

2. 16515600

1. 65721400

2. 29162100

0. 35483600

$-0.16101300$

0. 48772400

$-1.43116900$

$-2.27010300$

$-3.27900600$

$-1.81485200$

$-2.46940300$

$-0.48755300$

0.00742100

$-0.48833900$

$-1.81579700$

$-2.46993700$

-2. 27171200

-3. 28073400

$-1.43329700$

$-1.81524700$

$-0.16298200$

0. 48538400

0. 35353200

1. 65602900

2. 29001100

2. 16458000

3. 50198400

4. 12248200

3. 98521200

5. 00186700
0. 44026300

0. 93267700

1. 57463700

1. 97379000

2. 39741600

1. 90385900

1. 36931500

1. 31514200

0. 86605900

0. 31012300

$-0.18707100$

$-0.76418000$

$-0.80254600$

$-1.25508400$

$-1.69162400$

-1. 19904200

$-0.65365500$

$-0.62116200$

$-0.12412500$

$-0.44015400$

0. 12444200

0. 65366900

0. 62092900

1. 19913600

1. 59483500

1. 25559000

1. 69214000

0. 76503100

0. 80375100

0. 18779300

$-0.30919600$

$-0.25938100$

$-0.86536700$

$-1.36842200$

$-1.31405500$

$-1.90307500$

$-2.27972900$
1. 51120300

2. 28069100

0. 26060100

$-1.59498000$

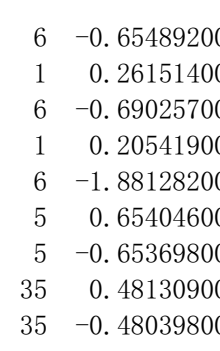
3. 15730400
3. 55509900
1. 87489900
1. 25868300
1. 32766700
$-0.87820900$
$-0.87781200$
$-2.17020500$
$-2.16862300$

64

3_1

62.39946000

63.56237400

$6 \quad 3.65929700$

12.82439000

64.78420100

14.82681500

$6 \quad 5.90689300$

$1 \quad 6.78787300$

$6 \quad 5.86745900$

16.71438700

64.71127900

64.69057700

15.56091300

$6 \quad 3.58612400$

63.58614600

14.47743500

$6 \quad 2.50371900$

$1 \quad 2.51321800$

$6 \quad 1.35181100$

10.49208000

$6 \quad 1.32358600$

$1 \quad 0.44268300$

62.42980100

$6-1.95000900$

$6-2.91920500$

$6-2.61754100$

$1-1.60753300$

$6-3.56932400$

$1-3.29436400$

$6-4.91601600$

$1-5.65630800$

$6-5.26136800$

$1-6.28442900$

$6-4.29260400$

$6-4.68334600$

$1-5.72066200$

$6-3.78109800$

$6-4.22585700$

$1-5.26726700$

$6-3.37763900$

$1-3.72327400$

$6-2.02490200$

$1-1.34826200$

$6-1.56438800$

$1-0.52614100$

$6-2.40397200$

$5 \quad 1.04135600$

$5-0.39508900$

$35 \quad 1.08816500$

$35 \quad 0.35324600$

$7-0.23992300$

$7 \quad 0.79748400$

$7 \quad 1.71647200$

$6-1.18577700$

$6-1.84730100$

$6-1.41843700$

$\begin{array}{ll}6 & -2.77094100\end{array}$

$-0.15749500$

$-0.54143500$

$-1.77574600$

$-2.46403300$

$-2.10760100$

$-3.05420400$

$-1.23214000$

$-1.51259300$

$-0.05129600$

0. 62930000

0. 32438600

1. 52946900

2. 18117000

1. 90587500

3. 12842800

3. 74911000

3. 50352500

4. 43145100

2. 66526200

2. 96189800

1. 49074300

0. 86233600

1. 05225200

$-0.64631900$

$-0.48604800$

0. 06922200

0. 38492200

0. 22021900

0. 65071200

$-0.17973800$

$-0.06107200$

$-0.70035000$

$-1.00335500$

$-0.85348900$

$-1.34385800$

$-1.63130700$

$-1.43638500$

$-1.86764100$

$-2.15884400$

$-1.89334100$

$-2.21313300$

$-1.48603600$

$-1.49001500$

$-1.10174600$ 


\begin{tabular}{|c|c|c|c|}
\hline 6 & -2.32900100 & 4. 27912500 & 1. 20241400 \\
\hline 6 & -3.00786100 & 4. 34759600 & -0.01082500 \\
\hline 1 & -1.65891800 & 1. 62994200 & -1.55457200 \\
\hline 1 & -0.90716900 & 3. 17868200 & 2. 39164000 \\
\hline 1 & -3.30096900 & 3. 44187200 & -1.94256700 \\
\hline 1 & -2.51413200 & 5.01429200 & 1. 97796600 \\
\hline 1 & -3.72614100 & 5. 14143000 & -0.18548500 \\
\hline \multicolumn{4}{|c|}{64} \\
\hline \multicolumn{4}{|c|}{$31^{\prime}$} \\
\hline 6 & -1.85342300 & 0.95338800 & 0.12106600 \\
\hline 6 & -3.02598500 & 0.62156400 & 0.83952700 \\
\hline 6 & -3.00050100 & 0.25134800 & 2. 22478700 \\
\hline 1 & -2.05257300 & 0.23278600 & 2. 75046700 \\
\hline 6 & -4.14268700 & -0.08466800 & 2. 89125700 \\
\hline 1 & -4.09252100 & -0.35859400 & 3. 94042000 \\
\hline 6 & -5.40417900 & -0.08262800 & 2. 22861000 \\
\hline 1 & -6.30030600 & -0.34941000 & 2. 77984900 \\
\hline 6 & -5.47743900 & 0.24965600 & 0.90974900 \\
\hline 1 & -6.42924700 & 0.25009100 & 0.38533600 \\
\hline 6 & -4.30095400 & 0.60241500 & 0.17499900 \\
\hline 6 & -4.37426100 & 0.91236800 & -1.18218900 \\
\hline 1 & -5.34023500 & 0.89164100 & -1.68237000 \\
\hline 6 & -3.23167000 & 1. 22609400 & -1.91699100 \\
\hline 6 & -3.30770900 & 1. 47492500 & -3.32387600 \\
\hline 1 & -4.28696700 & 1. 45458800 & -3.79521600 \\
\hline 6 & -2.18562000 & 1. 71928600 & -4.05911800 \\
\hline 1 & -2.25423100 & 1. 89934200 & -5.12710700 \\
\hline 6 & -0.91267700 & 1. 74754500 & -3.41822900 \\
\hline 1 & -0.02071500 & 1. 95068000 & -4.00315400 \\
\hline 6 & -0.80757700 & 1. 53387500 & -2.07299000 \\
\hline 1 & 0.17700900 & 1. 57666800 & -1.61776400 \\
\hline 6 & -1.95484000 & 1. 24882600 & -1.25952800 \\
\hline 6 & 2. 36854100 & -0.04231000 & 0.08118100 \\
\hline 6 & 3. 19354200 & -1.20861700 & 0.03686200 \\
\hline 6 & 2. 68821300 & -2.54863600 & 0.17672300 \\
\hline 1 & 1. 63309800 & -2.71147300 & 0.34234400 \\
\hline 6 & 3.50075200 & -3.64428000 & 0.11575800 \\
\hline 1 & 3. 06781800 & -4.63382000 & 0.22522200 \\
\hline 6 & 4. 90290800 & -3.51261700 & -0.08113600 \\
\hline 1 & 5. 53202000 & -4.39600300 & -0.11640600 \\
\hline 6 & 5. 43590200 & -2.26914500 & -0.22693600 \\
\hline 1 & 6.50198400 & -2.13188700 & -0.38676400 \\
\hline 6 & 4. 61061600 & -1.10091000 & -0.18630500 \\
\hline 6 & 5. 18470700 & 0.15324500 & -0.37628200 \\
\hline 1 & 6. 25861900 & 0.22978100 & -0.53259700 \\
\hline 6 & 4. 40879900 & 1. 30527000 & -0.39737700 \\
\hline 6 & 5.02674800 & 2.57265000 & -0.64844200 \\
\hline 1 & 6. 10411200 & 2. 59213100 & -0.78937900 \\
\hline 6 & 4. 29023000 & 3. 71391000 & -0.71936000 \\
\hline 1 & 4. 76439200 & 4. 67071900 & -0.91249500 \\
\hline 6 & 2. 87964500 & 3. 63984500 & -0.54208200 \\
\hline 1 & 2. 28223600 & 4. 54401300 & -0.60658400 \\
\hline 6 & 2.26657500 & 2. 44939900 & -0.28348100 \\
\hline 1 & 1. 19821500 & 2. 45615300 & -0.14538500 \\
\hline 6 & 2. 98502200 & 1. 21067500 & -0.18447000 \\
\hline 5 & -0.42579000 & 0.96842400 & 0.78066200 \\
\hline 5 & 0.79432000 & -0.18072900 & 0.42753100 \\
\hline 35 & -0.16243500 & 2. 35307600 & 2. 16832700 \\
\hline 35 & 0.51153000 & -1.19725500 & 2. 26358900 \\
\hline 7 & 0.15513000 & -1.19462000 & -0.79760000 \\
\hline 7 & 0.81757000 & -1.13893800 & -1.86976200 \\
\hline 7 & 1. 43496700 & -1.05823500 & -2.79798200 \\
\hline 6 & -1.10636100 & -1.91278100 & -0.96827200 \\
\hline 6 & -1.54165500 & -2.78153400 & 0.02604800 \\
\hline 6 & -1.83911900 & -1.72280100 & -2.13846800 \\
\hline 6 & -2.75147500 & -3.44836800 & -0.15370400 \\
\hline 6 & -3.03493600 & -2.40954400 & -2.30756000 \\
\hline 6 & -3.49820100 & -3.26737100 & -1.31300400 \\
\hline
\end{tabular}

$\begin{array}{rrr}1 & -0.95547200 & -2.92930100 \\ 1 & -1.49593100 & -1.02499500 \\ 1 & -3.10258800 & -4.11923000 \\ 1 & -3.61031700 & -2.25157900 \\ 1 & -4.43865000 & -3.79240600\end{array}$

0. 92257400

$-2.89836400$

0.62307500

$-3.21396100$

64

3_2P

$6 \quad 1.92955300$

0. 14543300

$-0.55197400$

$-0.42758700$

0.17176600

$-1.08594000$

$-0.99395300$

$-1.86179100$

$-2.37593800$

$-1.96804800$

$-2.54777100$

$-1.29177500$

$-1.18407200$

$-1.78499400$

0. 30094100

0. 90288100

0.31549100

2. 27866700

2. 77006700

3. 01312600

4. 08021100

2. 39887000

2. 98509000

1. 03581200

0. 39398500

$-0.50692700$

$-1.81957000$

$-2.12223100$

$-2.68066500$

$-3.67099100$

$-2.29077500$

$-2.98674600$

$-1.04955900$

$-0.73675200$

$-0.12349600$

1. 14075800

1. 42660400

2. 04104800

3. 32707800

3. 59789500

4. 18808200

5. 16081400

3. 81304500

4. 50438200

2. 59876800

2. 32737000

1. 66243900

0. 61066000

0.06639400

2. 41193600

$-0.87437400$

$-0.95849700$

$-0.94296900$

$-1.63197300$

$-1.90752400$

$-1.44109400$

$-3.27025000$

$-2.36180400$

$-4.17949900$

$-3.72564400$

$-0.37324200$

$-3.60139300$
$-1.44296300$

$-0.07425700$

$-0.63839800$

$-1.93705400$

$-2.66576600$

$-2.28114600$

$-3.29151800$

$-1.34929500$

$-1.63851200$

$-0.03950700$

0. 70295900

0. 30000900

1. 68085400

2. 44066500

2. 01370100

3. 12801600

3. 84961600

3. 30354500

4. 16773500

2. 37895700

2. 52272600

1. 25864400

0. 54146300

1. 07444800

$-0.19476400$

$-0.63339400$

$-1.11359800$

$-1.16352800$

$-1.52096600$

$-1.87614100$

$-1.49544600$

$-1.83366900$

$-1.05373900$

$-1.03208900$

$-0.60460200$

$-0.13306000$

$-0.11305600$

0. 32405300

0. 83531900

0.84170100

1. 30708500

1. 69283600

1. 29848100

1. 68056800

0. 81091400

0. 81310100

0. 29394700

$-1.05221700$

-0. 20237700

$-1.93574200$

$-2.55444400$

0. 70483600

0. 79696500

1. 61077700

1. 52532300

2. 51363200

1. 27251100

3. 25945900

2. 03005600

3. 02120400

2. 68105700

0. 48470800 


\begin{tabular}{|c|c|c|c|}
\hline 1 & -3.08664300 & -2.00806100 & 4. 02633900 \\
\hline 1 & -1.29147900 & -5.24248200 & 1. 83958000 \\
\hline 1 & -2.83894400 & -4.43862900 & 3. 60508200 \\
\hline \multicolumn{4}{|l|}{64} \\
\hline \multicolumn{4}{|c|}{ 3_2a } \\
\hline 6 & -1.89913100 & 1. 20146900 & -0.26776500 \\
\hline 6 & -1.20681800 & 2. 40666700 & -0.50883000 \\
\hline 6 & 0. 19681800 & 2. 42794700 & -0.79903800 \\
\hline 1 & 0.75467500 & 1. 49270800 & -0.83326000 \\
\hline 6 & 0.85307200 & 3. 59864800 & -1.03664500 \\
\hline 1 & 1. 91929100 & 3. 58560200 & -1.24065000 \\
\hline 6 & 0.15211700 & 4. 84044700 & -1.01362700 \\
\hline 1 & 0.69094300 & 5. 76222400 & -1.20861100 \\
\hline 6 & -1.18489700 & 4. 86714000 & -0.75116600 \\
\hline 1 & -1.73056200 & 5. 80675000 & -0.73367900 \\
\hline 6 & -1.90733800 & 3. 65908100 & -0.49086500 \\
\hline 6 & -3.27647600 & 3. 67096600 & -0.22387300 \\
\hline 1 & -3.80674300 & 4. 62042100 & -0.19836500 \\
\hline 6 & -3.98160200 & 2. 48930700 & 0.00808200 \\
\hline 6 & -5.38642700 & 2. 50399700 & 0.28072900 \\
\hline 1 & -5.89354400 & 3. 46455900 & 0.31265900 \\
\hline 6 & -6.07229500 & 1. 34613200 & 0.49546400 \\
\hline 1 & -7.13734100 & 1. 36928400 & 0.70259100 \\
\hline 6 & -5.39349700 & 0.09349400 & 0.44170900 \\
\hline 1 & -5.95273600 & -0.82389100 & 0.59784100 \\
\hline 6 & -4.05393800 & 0.03985400 & 0. 18922200 \\
\hline 1 & -3.55802000 & -0.92579700 & 0. 13150600 \\
\hline 6 & -3.28812700 & 1. 23176300 & -0.02911700 \\
\hline 6 & 2. 86789200 & -0.92413300 & 0.37462800 \\
\hline 6 & 3. 51644800 & 0.27098800 & 0.73274300 \\
\hline 6 & 3. 92140200 & 0.54360700 & 2. 07680100 \\
\hline 1 & 3. 75335400 & -0.21404600 & 2. 83505100 \\
\hline 6 & 4. 51281000 & 1. 73092300 & 2. 39411100 \\
\hline 1 & 4. 82497600 & 1. 92358100 & 3. 41552800 \\
\hline 6 & 4. 72381400 & 2. 73020200 & 1. 39818600 \\
\hline 1 & 5. 18762500 & 3. 67099400 & 1. 67672700 \\
\hline 6 & 4. 35434800 & 2. 50209200 & 0.10652800 \\
\hline 1 & 4. 52215800 & 3. 25139100 & -0.66254800 \\
\hline 6 & 3. 74837500 & 1. 26314900 & -0.27570300 \\
\hline 6 & 3. 38825900 & 1. 00032700 & -1.59940100 \\
\hline 1 & 3. 55796300 & 1.75819200 & -2.36090000 \\
\hline 6 & 2. 85187400 & -0.23372600 & -1.97319800 \\
\hline 6 & 2. 56420200 & -0.53873200 & -3.34164300 \\
\hline 1 & 2. 72913200 & 0.23640700 & -4.08455600 \\
\hline 6 & 2. 12059800 & -1.77462200 & -3.70663100 \\
\hline 1 & 1. 91773700 & -1.99842100 & -4.74888800 \\
\hline 6 & 1. 93681800 & -2.78797800 & -2.72117900 \\
\hline 1 & 1. 61204300 & -3.77758000 & -3.02645900 \\
\hline 6 & 2. 15463500 & -2.52344900 & -1.40178800 \\
\hline 1 & 2. 02740600 & -3.30345800 & -0.66212700 \\
\hline 6 & 2. 60029400 & -1.23554800 & -0.97537300 \\
\hline 5 & -1.08373000 & -0.12984200 & -0.28127900 \\
\hline 5 & 0.01357600 & -0.63346200 & 0.94527000 \\
\hline 35 & -1.18339200 & -1.20875100 & -1.89618800 \\
\hline 35 & 0.55971400 & 0.70027100 & 2. 26066700 \\
\hline 7 & 0.43219900 & -1.99601000 & 1. 12340600 \\
\hline 7 & 1. 63402000 & -2.43317200 & 1. 73213500 \\
\hline 7 & 2. 70194400 & -1.90575500 & 1. 40148500 \\
\hline 6 & -0.54476800 & -3.02802900 & 1. 06375300 \\
\hline 6 & -1.87139800 & -2.73224000 & 1. 39381300 \\
\hline 6 & -0.19995500 & -4.32922100 & 0.68976000 \\
\hline 6 & -2.85921300 & -3.70242500 & 1. 25908200 \\
\hline 6 & -1.18845800 & -5.30125600 & 0.59080900 \\
\hline 6 & -2.52201300 & -4.98906800 & 0.85060900 \\
\hline 1 & -2.12471100 & -1.75152100 & 1. 79016200 \\
\hline 1 & 0.83401900 & -4.58084200 & 0.48289200 \\
\hline 1 & -3.88759300 & -3.45469400 & 1. 50389600 \\
\hline 1 & -0.91388500 & -6.30951000 & 0. 29799300 \\
\hline
\end{tabular}

$$
\begin{aligned}
& 1 \\
& 64 \\
& 3 \_2 b
\end{aligned}
$$$$
\text { 3_2b }
$$$$
62.00270700
$$$$
\begin{array}{lll}
6 & 3.12418500 & -1.43366300
\end{array}
$$$$
\begin{array}{lll}
6 & 3.13297300 & -2.56703300
\end{array}
$$$$
1 \quad 2.25444200 \quad-3.20256800
$$$$
\begin{array}{lll}
6 & 4.22724200 & -2.87110200
\end{array}
$$$$
\begin{array}{lll}
6 & 4.20548200 & -3.73479900
\end{array}
$$$$
\begin{array}{lll}
6 & 5.40383800 & -2.06984500
\end{array}
$$$$
1 \quad 6.26199700 \quad-2.32685800
$$$$
\begin{array}{lll}
6 & 5.44918200 & -0.99709200
\end{array}
$$$$
\begin{array}{lll}
1 & 6.34335000 & -0.38367700
\end{array}
$$$$
\begin{array}{lll}
6 & 4.32429500 & -0.65050800
\end{array}
$$$$
\begin{array}{lll}
6 & 4.37701600 & 0.43042700
\end{array}
$$$$
\begin{array}{lll}
1 & 5.28954500 & 1.01951700
\end{array}
$$$$
\begin{array}{lll}
6 & 3.28931400 & 0.77330900
\end{array}
$$$$
\begin{array}{lll}
6 & 3.36479400 & 1.87069500
\end{array}
$$$$
\begin{array}{lll}
1 & 4.29554000 & 2.42898800
\end{array}
$$$$
\begin{array}{lll}
6 & 2.29853400 & 2.20752900
\end{array}
$$$$
\begin{array}{lll}
1 & 2.36408200 & 3.03690600
\end{array}
$$$$
\begin{array}{lll}
6 & 1.07820900 & 1.47911700
\end{array}
$$$$
\begin{array}{lll}
1 & 0.22677700 & 1.76285500
\end{array}
$$$$
\begin{array}{lll}
6 & 0.96787000 & 0.42851600
\end{array}
$$$$
\begin{array}{lll}
1 & 0.01832400 & -0.09609100
\end{array}
$$$$
\begin{array}{lll}
6 & 2.07601900 & 0.01165300
\end{array}
$$$$
\begin{array}{lll}
6 & -2.71100900 & 0.79165000
\end{array}
$$$$
\begin{array}{lll}
6 & -3.40289500 & 0.21442900
\end{array}
$$$$
\begin{array}{lll}
6 & -2.84447800 & 0.16562800
\end{array}
$$$$
\begin{array}{lll}
1 & -1.86420000 & 0.60646000
\end{array}
$$$$
\begin{array}{lll}
6 & -3.52617100 & -0.41451600
\end{array}
$$$$
1-3.08787100 \quad-0.44261600
$$$$
6-4.81647500 \quad-0.98445900
$$$$
1-5.33986700 \quad-1.44273800
$$$$
\begin{array}{lll}
6 & -5.38661300 & -0.95220300
\end{array}
$$$$
\begin{array}{lll}
1 & -6.36982100 & -1.38169600
\end{array}
$$$$
\begin{array}{lll}
6 & -4.70371500 & -0.35300400
\end{array}
$$$$
6-5.26926400 \quad-0.31536300
$$$$
\begin{array}{lll}
1 & -6.25373800 & -0.75176800
\end{array}
$$$$
\begin{array}{lll}
6 & -4.60528000 & 0.26755000
\end{array}
$$$$
\begin{array}{lll}
6 & -5.18971100 & 0.30921300
\end{array}
$$$$
1-6.17514900 \quad-0.12747600
$$$$
\begin{array}{lll}
6 & -4.52718100 & 0.88272900
\end{array}
$$$$
\begin{array}{lll}
1 & -4.97792200 & 0.91117700
\end{array}
$$$$
\begin{array}{lll}
6 & -3.23564100 & 1.45542100
\end{array}
$$$$
1-2.72645300 \quad 1.91862400
$$$$
\begin{array}{lll}
1 & -2.64452800 & 1.43206700
\end{array}
$$$$
1-1.66145200 \quad 1.87478000
$$$$
\begin{array}{lll}
6 & -3.30220200 & 0.83387600
\end{array}
$$$$
\begin{array}{lll}
5 & 0.72115000 & -1.96378400
\end{array}
$$$$
5-1.30183200 \quad 1.33287000
$$$$
\begin{array}{lll}
35 & 0.25663800 & -3.12463300
\end{array}
$$$$
\begin{array}{lll}
35 & -0.44165400 & -1.99116700
\end{array}
$$$$
\begin{array}{lll}
7 & -0.14164000 & 1.76429500
\end{array}
$$$$
7-1.51012900 \quad 3.80830800
$$$$
\begin{array}{lll}
7 & -0.73186100 & 4.56580000
\end{array}
$$$$
6 \quad 1.10237500 \quad 2.30292100
$$$$
\begin{array}{lll}
6 & 2.00123300 & 1.74238800
\end{array}
$$$$
6 \quad 1.48785300 \quad 3.42970400
$$$$
\begin{array}{lll}
6 & 3.25942800 & 2.30557300
\end{array}
$$$$
\begin{array}{lll}
6 & 2.75056600 & 3.98292600
\end{array}
$$$$
\begin{array}{lll}
6 & 3.64140100 & 3.42619700
\end{array}
$$$$
1 \quad 1.69684800 \quad 0.86505200
$$$$
1 \quad 0.78632000 \quad 3.84790200
$$$$
1 \quad 3.95048200 \quad 1.85993600
$$$$
1 \quad 3.03876900 \quad 4.85318400
$$$$
14.62567400 \quad 3.86239000
$$

$-0.49252800$

0. 29653600

1. 17413000

1. 23681000

1. 93015700

2. 58702600

1. 86552300

2. 47791000

1. 02603200

0. 95393300

0.21317200

$-0.66758200$

$-0.73058900$

$-1.46928200$

$-2.38460800$

$-2.43828900$

$-3.16224800$

$-3.85903600$

$-3.04717200$

$-3.65887200$

$-2.18264900$

$-2.10347400$

$-1.37550900$

0. 26092400

1. 34622500

2. 66284300

2. 83010300

3. 69264000

4. 68519400

3. 48160200

4. 31447500

2. 24391600

2. 07236500

1. 13852200

$-0.13769700$

$-0.29296100$

$-1.21901700$

$-2.52432200$

$-2.66133500$

$-3.56828300$

$-4.55501700$

$-3.37016400$

$-4.20977800$

$-2.14057900$

$-1.99171100$

$-1.01874500$

-0. 43120600

0. 46459000

$-1.90741100$

1. 10817700

0. 62454000

2. 43882900

2. 27176800

0. 79929200

1. 72000700

0. 06005600

1. 89251500

0. 23943600

1. 15425000

2. 28230700

$-0.65509200$

2. 60194900

$-0.34264500$

1. 29269600 
3_2c

$6 \quad 2.46260200$

63.16633900

6 2. 87406000

2. 09028900

63.56971500

13.33093000

64.61559000

$1 \quad 5.15638200$

64.93826500

$1 \quad 5.73960100$

64.23739500

64.58833800

$1 \quad 5.39632700$

63.94156300

$6 \quad 4.34182700$

15.15375700

$6 \quad 3.73231200$

14.04865600

$6 \quad 2.67955900$

$1 \quad 2.20628200$

62.26218200

11.46905100

62.86596300

$\begin{array}{ll}6 & -2.49624600\end{array}$

$\begin{array}{ll}6 & -2.35475200\end{array}$

$6-1.08800400$

$1-0.19200000$

$6-0.99838300$

$1-0.02855700$

$6-2.16459200$

$1-2.06956800$

$6-3.38785300$

$1-4.28308600$

$\begin{array}{ll}6 & -3.52954700\end{array}$

$6-4.77761700$

$1-5.66384100$

$\begin{array}{ll}6 & -4.91351400\end{array}$

$\begin{array}{ll}6 & -6.19084500\end{array}$

$1-7.06716700$

$6-6.30536400$

$1-7.27882600$

$6-5.14426300$

$1-5.25032500$

$\begin{array}{ll}6 & -3.90778700\end{array}$

$1-3.02774200$

$6-3.74814700$

$5 \quad 1.23733100$

$5-0.34748100$

$35 \quad 1.59019000$

$35-0.75628200$

$\begin{array}{ll}7 & -1.32753800\end{array}$

$7 \quad 1.40442200$

$7 \quad 1.08083000$

$\begin{array}{ll}6 & -1.14094600\end{array}$

$\begin{array}{ll}6 & -0.93475600\end{array}$

$6-1.14461400$

$6-0.69186400$

$6-0.90264800$

$6-0.67169000$

$1-0.95910300$

$1-1.31628100$

$1-0.52477300$

$1-0.88063500$

$1-0.48302400$ 44

Mes2B2C12

$6-1.85499300$
$-0.21287000$

$-1.38475600$

$-2.66428400$

$-2.74619900$

$-3.78165900$

$-4.73605900$

$-3.70925900$

$-4.60970900$

$-2.51284000$

$-2.43894300$

$-1.31698400$

$-0.08684800$

$-0.04011400$

1. 08917000

2. 35616200

2. 37607200

3. 50802800

4. 46507400

3. 46010400

4. 38113400

2. 26816400

2. 26332400

1. 03009200

$-0.11817400$

$-0.45256600$

$-0.46262200$

$-0.20723700$

$-0.78063300$

$-0.77873500$

$-1.11817800$

$-1.37255500$

$-1.12065900$

$-1.37513900$

$-0.78611400$

$-0.77222700$

$-1.02137300$

$-0.44773400$

$-0.43996200$

$-0.68763600$

$-0.13422500$

$-0.13198700$

0. 17731800

0. 40578400

0. 18415800

0. 41204400

$-0.11863200$

$-0.30135200$

$-0.63297700$

$-0.21127500$

$-2.55910100$

0. 26516800

1. 52079300

2. 56037800

1. 68028000

2. 19641400

2. 52900300

3. 55837600

3. 88673100

4. 40479600

1. 52871000

2. 11944200

3. 95497600

4. 53849700

5. 46562100

0. 29338200
$-0.40744900$

$-0.04880600$

$-0.62546000$

$-1.37220000$

$-0.26774100$

$-0.72596600$

0. 69813300

0. 97116900

1. 26375000

1. 99406500

0. 90758200

1. 46070100

2. 18759200

1. 08317700

1. 61392400

2. 33613000

1. 21529600

1. 61777600

0. 25562600

$-0.06973400$

$-0.26240900$

$-1.00504500$

0. 13108800

0. 31874900

1. 67536400

2. 34304500

1. 78466000

3. 66642400

4. 15466500

4. 41576200

5. 46646600

3. 81506100

4. 37573400

2. 43057600

1. 80732800

2. 38626800

0. 45667500

$-0.18749900$

0. 40494200

$-1.51081200$

$-1.99059800$

$-2.27841100$

-3. 33416700

$-1.70247300$

$-2.29356800$

$-0.31379500$

$-1.38527100$

$-0.87307100$

$-3.30624900$

$-0.81213400$

$-0.42859600$

3. 05201200

2. 90182200

$-0.59875700$

$-1.87838700$

0. 50911200

$-2.04492900$

0. 33476200

$-0.93958800$

$-2.73441400$

1. 49976500

$-3.04116300$

1. 20243100

$-1.07002800$

$-0.35091500$ $\begin{array}{ll}6 & -2.85523600\end{array}$

$6-3.88794100$

0. 53339300

$-0.39147600$

$-1.55727600$

$-1.78556500$

$-0.87706200$

0. 17927600

$-1.07144100$

$-2.03930400$

$-1.80504800$

$-0.56449800$

0. 43305000

1. 27154300

1. 26670200

2. 43399400

2. 56978100

1. 76409100

1. 64655600

2. 65645400

1. 96248700

$-1.14939300$

$-0.90182500$

$-2.20307200$

$-0.55650100$

$-2.69405500$

$-0.20008800$

$-2.53774700$

-2. 40348600

$-3.56943700$

-2. 40804700

$-2.87994500$

$-3.23155600$

$-3.74769900$

$-2.51788700$

$-1.37192700$

$-1.63456400$

$-2.20981300$

$-0.51406700$

1. 78081300

1. 72695000

2. 17099100

2. 51727000

$-3.00125400$

$-0.36271200$

60

$1-4.86157400$

8_1(4_1)

$6-2.32403400$

$-0.75916300$

$-1.56340800$

$-1.09153300$

$6-4.39964600$

0. 15246100

0.90878500

0. 47359900

1. 00629100

1. 91455100

3. 28930100

3. 82263300

2. 93372300

1. 55789800

$-1.22688900$

$-0.60569500$

$-2.43830400$

$-1.37300400$

$-2.95919900$

$-3.04738500$

$-3.67894600$

$-3.26313600$

1. 34906100

0. 80017100

2. 19361500

0. 60554600

0. 76215200

$-0.00083200$

$-0.94115300$

$-1.13236400$

0. 35372800

0.98137100

0. 84492700

0. 09337600

$-0.52367300$

$-0.39886400$

$-0.51408100$

0. 47050500

$-1.86766400$

1. 67440800

1. 47757900

2. 27968400

0. 90665500 


\begin{tabular}{|c|c|c|c|}
\hline 1 & -2.70408800 & 1. 75416200 & 2. 61699200 \\
\hline 1 & -4.45046800 & 1. 86458300 & 0.85888500 \\
\hline 1 & -5.02167400 & -1.71423300 & -1.41866700 \\
\hline 6 & -6.22555500 & 0.64722700 & -0.82046300 \\
\hline 1 & -6.99722500 & -0.11611700 & -0.68247200 \\
\hline 1 & -6.52900600 & 1. 54076100 & -0.26951000 \\
\hline 1 & -6.20778800 & 0.90108900 & -1.88572200 \\
\hline 6 & 1. 20746300 & 5. 30295800 & 0.35544200 \\
\hline 1 & 0.71211800 & 5. 85790300 & -0.44483600 \\
\hline 1 & 0.83739400 & 5. 68504800 & 1. 31209800 \\
\hline 1 & 2. 27777200 & 5. 53012100 & 0.30944700 \\
\hline 6 & -0.85418000 & 1. 50512900 & -2.14855200 \\
\hline 1 & -1.43471400 & 2. 36780300 & -2.48665200 \\
\hline 1 & -0.26577400 & 1. 14469500 & -2.99746500 \\
\hline 1 & -1.55394100 & 0.71181400 & -1.87958700 \\
\hline 6 & 1. 63964900 & 0.72741000 & 2. 28841700 \\
\hline 1 & 1. 57485900 & 1. 33423500 & 3. 19571000 \\
\hline 1 & 0.99916300 & -0.14585000 & 2. 44481200 \\
\hline 1 & 2. 67250800 & 0.36793600 & 2. 21938400 \\
\hline 1 & -0.18498300 & 3. 96588400 & -1.57357300 \\
\hline 1 & 1.91510800 & 3. 32536800 & 2. 08503200 \\
\hline 14 & 3. 51064200 & -0.69940200 & -0.80328800 \\
\hline 7 & 1. 98317800 & -1.13980800 & 0.18617400 \\
\hline 7 & 2. 17773500 & -2.06374000 & 1. 00139800 \\
\hline 7 & 2. 39686000 & -2.87173800 & 1. 74626900 \\
\hline 6 & 4. 78808500 & -0.28216100 & 0.49215400 \\
\hline 6 & 3. 93044200 & -2.28509600 & -1.68929600 \\
\hline 6 & 3. 13490200 & 0.72689800 & -1.92697100 \\
\hline 1 & 5.77625800 & -0.23062300 & 0.02154500 \\
\hline 1 & 4. 59252300 & 0.69079900 & 0.95227800 \\
\hline 1 & 4. 85017800 & -1.03530100 & 1. 28516700 \\
\hline 1 & 3. 13151600 & -2.56357900 & -2.38191800 \\
\hline 1 & 4. 85672200 & -2.16528900 & -2.26113700 \\
\hline 1 & 4. 08316900 & -3.11447900 & -0.98987500 \\
\hline 1 & 2. 98639300 & 1. 65884200 & -1.37602900 \\
\hline 1 & 4. 00016000 & 0.85191200 & -2.58986300 \\
\hline 1 & 2. 25520000 & 0.53779900 & -2.54593600 \\
\hline \multicolumn{4}{|c|}{46} \\
\hline \multicolumn{4}{|c|}{ 8_2（4_2) } \\
\hline 6 & -3.34051400 & -1.86365700 & 1. 68379400 \\
\hline 6 & -4.10931100 & -2.55086300 & 0.70831400 \\
\hline 6 & -5.46972600 & -2.75924200 & 0.92378900 \\
\hline 6 & -6.10933500 & -2.33818900 & 2. 08913500 \\
\hline 6 & -5.35304500 & -1.66540700 & 3. 04494700 \\
\hline 6 & -3.99680600 & -1.40714300 & 2. 85410400 \\
\hline 6 & -1.08471900 & 1. 24983600 & 1. 63591300 \\
\hline 6 & -1.48635400 & 1. 49884200 & 0.30095900 \\
\hline 6 & -1.62273100 & 2. 80459400 & -0.16468600 \\
\hline 6 & -1.39499400 & 3. 90093300 & 0.66417600 \\
\hline 6 & -1.02042600 & 3. 65815900 & 1. 98387900 \\
\hline 6 & -0.84958600 & 2. 36532600 & 2. 48033800 \\
\hline 5 & -1.81510800 & -1.57775600 & 1. 54878600 \\
\hline 5 & -0.93568500 & -0.22825900 & 2. 13388100 \\
\hline 17 & -0.73221500 & -2.83809300 & 0.85085900 \\
\hline 6 & -3.53270000 & -3.06684400 & -0.58971200 \\
\hline 1 & -2.80521400 & -2.38128300 & -1.03056300 \\
\hline 1 & -3.02498500 & -4.02478300 & -0.44333000 \\
\hline 1 & -4.33306300 & -3.22210100 & -1.31725100 \\
\hline 6 & -3.26579300 & -0.65505000 & 3. 94323300 \\
\hline 1 & -2.42098000 & -1.22392800 & 4. 34547800 \\
\hline 1 & -2.88930200 & 0.30749000 & 3. 57631000 \\
\hline 1 & -3.93711300 & -0.43986700 & 4. 77772600 \\
\hline 1 & -5.83244600 & -1.32636800 & 3. 96034800 \\
\hline 1 & -6.04939800 & -3.27325800 & 0.15972500 \\
\hline 6 & -7.57912000 & -2.59113000 & 2. 28940500 \\
\hline 1 & -8.17766000 & -1.95854400 & 1. 62543100 \\
\hline 1 & -7.83328800 & -3.63079700 & 2. 06318900 \\
\hline 1 & -7.88310400 & -2.37962200 & 3. 31707100 \\
\hline
\end{tabular}

$\begin{array}{rr}6 & -1.52277300 \\ 1 & -1.82904200 \\ 1 & -0.56372300 \\ 1 & -2.25288400 \\ 6 & -1.77020600 \\ 1 & -2.66709700 \\ 1 & -1.95623100 \\ 1 & -0.93588300 \\ 6 & -0.43389200 \\ 1 & -0.53129900 \\ 1 & -1.03870600 \\ 1 & 0.60558300 \\ 1 & -1.91957700 \\ 1 & -0.85184500 \\ 7 & 0.07165300 \\ 7 & 0.25059500 \\ 7 & 0.49863700\end{array}$

5. 30669500

5. 99741800

5. 66146200

5. 36501500

0. 37276500

$-0.18362500$

0.76050900

$-0.33175200$

2. 23378200

3. 19723700

1. 50150700

1. 90624300

2. 97269900

4. 50293400

-0. 46472900

$-1.58635200$

$-2.55159800$

46

8_3 ( 4_3

$6-3.52011100-2.29774400$

$6-3.65268700$

$\begin{array}{ll}6 & -4.87805100\end{array}$

$\begin{array}{ll}6 & -5.98293900\end{array}$

$\begin{array}{ll}6 & -5.83654500\end{array}$

$\begin{array}{ll}6 & -4.62554800\end{array}$

$\begin{array}{ll}6 & -1.27763900\end{array}$

$\begin{array}{ll}6 & -2.22328500\end{array}$

$6-1.90748300$

$\begin{array}{ll}6 & -0.67946200\end{array}$

$\begin{array}{ll}6 & 0.24507000\end{array}$

$\begin{array}{ll}6 & -0.03259500\end{array}$

$5-2.14689200$

$5-1.60304900$

$17-0.82990100$

$\begin{array}{rr}6 & -2.46977000\end{array}$

$1-1.84774500$

$1-1.83238700$

$1-2.79282000$

$6-4.49380000$

$1-3.73620800$

$1-4.18860300$

$1-5.43766800$

$1-6.68391700$

$1-4.97400500$

$\begin{array}{ll}6 & -7.29679100\end{array}$

$1-7.76639800$

$1-7.15704400$

$\begin{array}{ll}1 & -7.99690500\end{array}$

$\begin{array}{ll}6 & -0.35073100\end{array}$

10.36502000

$1 \quad 0.09580000$

$1-1.24770400$

$\begin{array}{ll}6 & -3.54974200\end{array}$

$1-4.06505700$

$1-4.20480600$

$1-3.41733800$

6.96984100
1

$1 \quad 1.93303900$

$1 \quad 0.62138200$

11.13059800

$1-2.63236700$

$1 \quad 1.20097400$

$\begin{array}{ll}7 & -1.87179500\end{array}$

$\begin{array}{ll}7 & -3.56486700\end{array}$

$\begin{array}{ll}7 & -3.39270700\end{array}$ 46

$-3.29449700$

$-3.93882500$

$-3.62214300$

$-2.63555300$

$-1.96664100$

2. 38561700

3. 18875700

4. 51709800

5. 06424200

4. 25449400

2. 92069100

$-1.58202100$

0. 94249900

$-2.54355600$

$-3.71237200$

$-2.85736000$

$-4.41010500$

$-4.21261200$

$-0.93533800$

$-1.23851900$

0. 03665300

$-0.80309200$

$-2.38187600$

$-4.70989800$

$-4.33048200$

$-4.02279400$

$-5.41464800$

$-4.11205700$

6. 49694800

6. 91070200

6. 57446000

7. 12118600

2. 61465500

2. 13618100

3. 39038800

1. 85082900

2. 07043300

2. 57840400

1. 84493400

1. 11277900

5. 14388100

4. 67517600

$-0.25809000$

$-4.57994400$

$-5.32305100$

8_3'
0. 14246500

0. 93233300

$-0.25072500$

$-0.66882100$

$-0.66509300$

$-0.36690800$

$-1.66956500$

$-0.73623000$

3. 92799500

4. 43453000

4. 47007300

4. 01475700

$-1.19772300$

2. 64853900

3. 15826300

3. 64562000

4. 16825100

2. 35265700

1. 37047600

1. 20473200

1. 99705500

2. 97090500

3. 15566700

1. 66300400

0. 99181100

0. 72484900

1. 10501100

1. 76773200

2. 05363200

2. 59592300

1. 98837400

3. 38224400

0. 53183100

0. 24672700

1. 08683800

$-0.38454300$

4. 24966000

4. 98219600

3. 84978700

4. 78361300

3. 60461800

0. 44214400

1. 79225900

0. 85197900

1. 74638800

2. 60216100

0. 78303700

1. 49736500

$-0.21435400$

0. 79023300

0. 56598100

1. 40497600

0. 16384100

$-0.20748600$

2. 79067700

2. 87262000

3. 80431800

2. 28588300

0. 21082800

2. 07072800

2. 27816400

4. 62717900

3. 83585500 


\begin{tabular}{|c|c|c|c|}
\hline 6 & -4.33555100 & -0.76304100 & 0.83969600 \\
\hline 6 & -4.74851700 & -1.05165200 & -0.48510700 \\
\hline 6 & -6.08354400 & -0.87689700 & -0.84272800 \\
\hline 6 & -7.04399500 & -0.45064500 & 0.07372700 \\
\hline 6 & -6.63340500 & -0.17243200 & 1. 37604100 \\
\hline 6 & -5.30365100 & -0.30376200 & 1. 76874800 \\
\hline 6 & -0.85260800 & 2. 16119900 & 3. 56038800 \\
\hline 6 & -0.93063300 & 3. 47630600 & 3. 05941400 \\
\hline 6 & -0.27661900 & 4. 49029100 & 3. 75136000 \\
\hline 6 & 0.44194500 & 4. 24141800 & 4. 92362800 \\
\hline 6 & 0.49520800 & 2. 93225300 & 5. 39935800 \\
\hline 6 & -0.13930500 & 1. 88140000 & 4. 73866400 \\
\hline 5 & -2.86347600 & -0.89215600 & 1. 31009100 \\
\hline 5 & -2.07551800 & 0.25382000 & 2. 24226500 \\
\hline 17 & -1.86001000 & -2.29607200 & 0.86221400 \\
\hline 6 & -3.79243600 & -1.51319800 & -1.56052000 \\
\hline 1 & -2.84894700 & -0.96210000 & -1.54340500 \\
\hline 1 & -3.55112900 & -2.57427700 & -1.45100400 \\
\hline 1 & -4.24274600 & -1.37432600 & -2.54608900 \\
\hline 6 & -4.95237000 & 0.04081200 & 3. 19864100 \\
\hline 1 & -4.34008300 & -0.72844100 & 3. 67720400 \\
\hline 1 & -4.39932300 & 0.98484700 & 3. 25893600 \\
\hline 1 & -5.86102700 & 0.15595700 & 3. 79438800 \\
\hline 1 & -7.36837400 & 0.16500800 & 2. 10346800 \\
\hline 1 & -6.38411900 & -1.08057300 & -1.86832000 \\
\hline 6 & -8.48821500 & -0.31941900 & -0.32700300 \\
\hline 1 & -8.58442400 & -0.00537000 & -1.36949100 \\
\hline 1 & -9.00428300 & -1.28049400 & -0.22424900 \\
\hline 1 & -9.01135300 & 0.40484000 & 0.30215100 \\
\hline 6 & 1. 12541900 & 5. 36873400 & 5. 65290000 \\
\hline 1 & 1. 72306200 & 4. 99650600 & 6. 48831400 \\
\hline 1 & 1. 78904200 & 5. 92808700 & 4. 98623500 \\
\hline 1 & 0.39554800 & 6. 07955900 & 6. 05456100 \\
\hline 6 & -1.70442500 & 3. 75206600 & 1. 80149900 \\
\hline 1 & -2.74211900 & 3. 41471300 & 1. 89581700 \\
\hline 1 & -1.70731300 & 4. 81952000 & 1. 57076600 \\
\hline 1 & -1.27637100 & 3. 21597100 & 0.94765200 \\
\hline 6 & -0.08922800 & 0.47427000 & 5. 26120000 \\
\hline 1 & 0.51451900 & 0.41434200 & 6. 16949300 \\
\hline 1 & -1.09603600 & 0. 10967700 & 5. 49263000 \\
\hline 1 & 0.33412200 & -0.20817400 & 4. 51640500 \\
\hline 1 & -0.33007200 & 5.50670400 & 3. 36598200 \\
\hline 1 & 1. 04835000 & 2. 71791300 & 6. 31121300 \\
\hline 7 & -1.48257100 & 1. 14540200 & 2. 88603500 \\
\hline 7 & -2.10318400 & -2.25345100 & 4. 19147700 \\
\hline 7 & -1.14695400 & -2.67773300 & 4. 52710200 \\
\hline \multicolumn{4}{|l|}{88} \\
\hline \multicolumn{4}{|c|}{ 8_3-Di（4_3-Di } \\
\hline 6 & 2. 99423500 & -1.85172500 & -0.48361600 \\
\hline 6 & 3. 72672800 & -2.37934500 & 0.59411100 \\
\hline 6 & 5. 08317100 & -2.08026200 & 0.71559500 \\
\hline 6 & 5. 73943600 & -1.27993800 & -0.22070700 \\
\hline 6 & 5. 01457000 & -0.80883400 & -1.31332000 \\
\hline 6 & 3. 65477900 & -1.08216500 & -1.45713500 \\
\hline 6 & -2.15940300 & -1.32502000 & -0.23724000 \\
\hline 6 & -3.01870000 & -1.01147400 & -1.30453000 \\
\hline 6 & -4.30038200 & -1.55743100 & -1.33805200 \\
\hline 6 & -4.74993700 & -2.41417700 & -0.33406700 \\
\hline 6 & -3.87226800 & -2.74907400 & 0.69890700 \\
\hline 6 & -2.58176800 & -2.22538400 & 0.75706300 \\
\hline 5 & 1.43368100 & -1.98285300 & -0.50682000 \\
\hline 5 & -0.82163800 & -0.55583400 & -0.05395700 \\
\hline 17 & 0.64684900 & -3.51309300 & -1.02002300 \\
\hline 6 & 3. 05758700 & -3.25763300 & 1. 62453700 \\
\hline 1 & 2. 05114700 & -2.90468100 & 1. 87890300 \\
\hline 1 & 2. 95078200 & -4.28270900 & 1. 25288700 \\
\hline 1 & 3. 63863300 & -3.29744300 & 2. 54966400 \\
\hline 6 & 2. 92653900 & -0.56453500 & -2.67272300 \\
\hline
\end{tabular}

$\begin{array}{lr}1 & 3.02297200 \\ 1 & 1.85604900 \\ 1 & 3.33314600 \\ 1 & 5.51292300 \\ 1 & 5.63769400 \\ 6 & 7.17822700 \\ 1 & 7.24304600 \\ 1 & 7.75270600 \\ 1 & 7.66047300\end{array}$

$6-6.16075400$

$1-6.19098600$

$1-6.78092600$

$1-6.62457300$

$\begin{array}{ll}6 & -2.54949800\end{array}$

$1-2.05975200$

$1-3.38310800$

$1-1.82452100$

$\begin{array}{ll}6 & -1.65684000\end{array}$

$1-2.21886000$

$1-1.04403200$

$1-0.97031300$

$1-4.96625700$

$1-4.20662000$

$7 \quad 0.60011500$

$\begin{array}{ll}6 & -2.99062100\end{array}$

$\begin{array}{ll}6 & -3.74502300\end{array}$

$\begin{array}{ll}6 & -5.09350200\end{array}$

$\begin{array}{ll}6 & -5.71753900\end{array}$

$\begin{array}{ll}6 & -4.97038600\end{array}$

$\begin{array}{ll}6 & -3.62105000\end{array}$

$\begin{array}{ll}6 & 2.16395300\end{array}$

$6 \quad 2.48114900$

6 3. 75744900

64.72395000

$6 \quad 4.37903100$

63.11430400

$\begin{array}{ll}5 & -1.43337200\end{array}$

$5 \quad 0.83196700$

$\begin{array}{ll}17 & -0.66271900\end{array}$

$\begin{array}{ll}6 & -3.12303700\end{array}$

$1-2.06471900$

$1-3.18171000$

$1-3.63450600$

$\begin{array}{ll}6 & -2.84935100\end{array}$

$1-2.72196700$

$1-1.84501900$

$1-3.36529300$

$1-5.44555900$

$1-5.66528000$

$6 \quad-7.14520200$

$1-7.18089200$

$1-7.70587800$

$1-7.66254000$

$6 \quad 6.11598900$

$1 \quad 6.66330800$

1 6.68584500

$1 \quad 6.09616500$

$6 \quad 1.45327100$

10.72514700

$1 \quad 1.91984400$

10.89597500

$6 \quad 2.76195700$

13.64296700

12.32842400

$1 \quad 2.02494400$

14.00864200

15.11440800

$7-0.59036200$
$-1.26869900$

$-0.43265600$

0. 39754300

-0. 19340100

$-2.46557800$

$-0.88369000$

0.00124600

$-1.68145400$

$-0.63469700$

$-2.94199500$

$-3.99935700$

$-2.39794200$

$-2.83515500$

$-0.12956800$

$-0.72930100$

0. 40381700

0. 61595400

$-2.63747500$

$-2.93231300$

$-3.49372800$

$-1.83534300$

$-1.29762800$

$-3.42803900$

$-0.90906500$

1. 82969800

1. 05181700

0. 81399600

1. 33314000

2. 14166300

2. 40224100

1. 30535000

2. 19522600

2. 74975900

2. 46323700

1. 62056400

1. 03787500

1. 93069200

0. 50793600

3. 44091500

0. 49006200

0. 25088800

1. 21752300

$-0.42092200$

3. 28750500

4. 29225700

2. 89698400

3. 38708900

2. 56551400

0. 19060600

0. 97959600

0.11599200

0.71607000

1. 80495800

3. 02601300

2. 94174800

2. 48922400

4. 08068000

2. 55668400

3. 26593900

3. 02530800

1. 68224700

0. 17015400

$-0.33780500$

0.77704100

$-0.59534100$

3. 41786000

1. 40067000

0. 85266900
$-3.50717700$

$-2.48776500$

$-2.99760900$

$-2.06045000$

1. 56968600

$-0.02088100$

0. 62390600

0. 45792300

$-0.96981000$

$-0.33763800$

$-0.05934700$

0. 38416900

$-1.32162500$

$-2.43345500$

$-3.20958700$

$-2.89786300$

$-2.09025500$

1. 87759500

2. 76788000

1. 57316800

2. 17141400

$-2.15916600$

1. 48154400

$-0.12055500$

0. 54271600

1. 43846800

1. 17645700

0. 04346700

$-0.81394400$

$-0.57636900$

0. 30314500

$-0.74084400$

$-0.80081800$

0. 16630300

1. 22138700

1. 30168900

0. 68255400

0. 23174800

1. 27647500

2. 69271000

2. 55087500

3. 51064300

3. 01534700

$-1.52605100$

$-1.10837500$

$-1.72949100$

$-2.48463100$

$-1.69698300$

1. 86163600

$-0.27910500$

$-0.95418100$

0. 62159600

$-0.77589900$

0. 04534500

0. 98769900

$-0.72182800$

$-0.24423300$

$-1.78617100$

$-1.37569500$

$-2.65652600$

$-2.14156900$

2. 48286500

2. 88393000

3. 28631300

2. 21784400

$-1.62256700$

1. 99339500

0. 33065800 
60

8_4 (4_4

62.97194700

$6 \quad 3.20521300$

64.28420700

65.14746700

$6 \quad 4.90176200$

63.83184200

$6-1.76819200$

$6-1.42522000$

$6-2.30184600$

$6-3.51153200$

$6-3.82381400$

$6-2.96564400$

$5 \quad 1.72658300$

$5-0.78616200$

$17 \quad 2.10231800$

62.29984600

$1 \quad 1.28849200$

12.21365400

12.68183200

$6 \quad 3.61509700$

13.95196500

12.55528200

14.16567800

15.55573500

14.45759100

$6 \quad 6.32349700$

16.73101200

16.04327400

17.12937800

$6-4.43528400$

$1-5.37566300$

$1-4.66834800$

$1-3.97347900$

$6-0.11525000$

$1-0.07780300$

$1 \quad 0.05359200$

10.72690600

$6-3.31234300$

$1-2.80529200$

$1-2.99596100$

$1-4.38761300$

$1-2.03830000$

$1-4.75081700$

$7 \quad 0.43551600$

$7-1.45017400$

$7 \quad-0.83758700$

$7-0.24683900$

$14-2.73084100$

$6-2.32064600$

$1 \quad-2.96341300$

$1-1.28221700$

$1-2.49567700$

$6 \quad-2.45709100$

$1-3.15684300$

$1-2.63826100$

$1-1.44626200$

$6-4.40893100$

$1-5.17189400$

$1-4.57324100$

$1-4.55961900$

60

8_5 (4_5

$6 \quad 6.88447000$

$6 \quad 6.70423700$

$6 \quad 5.91669600$

$6 \quad 5.31479200$
0. 03469000

1. 05547600

0.96260000

$-0.13422600$

$-1.14683500$

$-1.07665500$

$-1.08612800$

$-1.85399300$

$-2.83394100$

$-3.09657700$

$-2.35917600$

$-1.36561700$

0. 11665800

0. 04042600

0. 34709200

2. 26306600

1. 97967100

2. 74247200

3. 00540500

$-2.19850400$

$-1.91112000$

$-2.46228300$

$-3.09530700$

$-2.01671200$

1. 76216900

$-0.20016200$

-1. 21257900

0. 11149800

0. 46302700

$-4.16782300$

$-4.18731100$

$-4.01073000$

$-5.15672800$

$-1.62439600$

$-2.17190500$

$-0.56333400$

$-1.95000300$

$-0.65302800$

$-1.13143900$

0. 39414700

$-0.68151100$

$-3.41224300$

$-2.56556500$

0. 05916500

1. 53002200

2. 42344600

3. 15424100

2. 08824500

1. 25203700

1. 66680500

1. 43356300

0. 17358800

3. 93461900

4. 37432900

4. 42215700

4. 19228300

1. 65283700

2. 09992100

0. 57191900

2. 04728600

2. 25127600

3. 50806800

4. 46583500

4. 21023300
0. 52341000

$-0.41831600$

$-1.29528300$

$-1.27588700$

$-0.35175900$

0. 54377900

0. 10960100

$-1.02443800$

$-1.48355200$

$-0.83326400$

0. 30592100

0. 78851100

1. 49480600

0. 59386300

3. 28974600

$-0.48146900$

$-0.79971600$

0. 49973700

$-1.18715800$

1. 53058900

2. 53192100

1. 61157000

1. 23414600

$-0.32871100$

$-2.01385700$

$-2.21570700$

$-2.27373200$

$-3.22633500$

$-1.88255800$

$-1.35135600$

$-0.79536600$

$-2.40904000$

$-1.26493300$

$-1.73736700$

$-2.68247500$

$-1.94861300$

-1. 11788600

2. 07366200

2. 91869400

2. 06587900

2. 27059100

$-2.36725400$

0. 83739200

1. 09869700

0. 35383800

0. 98858700

1. 59153100

$-0.87213000$

$-2.47879500$

$-3.26384000$

$-2.77231300$

$-2.43613800$

$-0.93514400$

$-1.65385900$

0. 02843800

$-1.26857200$

$-0.19948100$

$-0.84757400$

$-0.18572700$

0. 80976100

$-0.42593700$

0. 17790200

$-0.46004300$

$-1.69221400$
$6 \quad 5.55307200$

$6 \quad 6.33166100$

64.28424000

63.75113100

62.81782900

62.38505000

$6 \quad 2.88139500$

63.81699800

57.62134000

$5 \quad 5.44516500$

$17 \quad 9.39467800$

67.35719700

$1 \quad 7.38015100$

8. 39570100

6. 83250600

6. 59022900

6. 65422000

5. 79885100

7. 54048500

5. 10558100

5. 75557700

64.38467300

$1 \quad 3.37674900$

1. 41682300

14.30656900

$6 \quad 1.43553600$

10.71093600

0. 88466300

1. 98080000

4. 14922200

5. 17264000

14.07979800

3. 49109100

64.28726200

14.33207000

15.27612700

$1 \quad 3.59488500$

12.42719400

$1 \quad 2.53836100$

$7 \quad 6.87597900$

$7 \quad 5.41317600$

$7 \quad 6.70479600$

$7 \quad 7.53558000$

$14 \quad 4.13997500$

$6 \quad 2.49033500$

12.34242700

$1 \quad 2.36654000$

$1 \quad 1.68921000$

64.31356900

13.59390000

$1 \quad 5.31791200$

14.13927500

64.46907000

13.72950400

14.43438700

$1 \quad 5.45973100$

120

8_P

$6 \quad 1.93499000$

63.11281100

63.83282700

63.44636400

62.30764800

$6 \quad 1.57079500$

63.75206600

64.27458900

$6 \quad 5.57785100$

$6 \quad 6.38547900$

2. 98069600

2. 00158100

0. 51574900

1. 77453100

2. 36903700

1. 73431500

0. 46201500

$-0.15807200$

1. 12869400

$-0.10899400$

1. 04888700

3. 83234100

2. 97074900

4. 14950400

4. 64209200

0. 69269700

$-0.14223200$

0. 46383600

0. 72804100

2. 77158800

5. 42791200

5. 21651900

5. 11598000

6. 24129600

5. 07356900 


\begin{tabular}{|c|c|c|c|}
\hline 6 & 5. 86244300 & -0.13178200 & -0.35552000 \\
\hline 6 & 4. 56146100 & 0.22367900 & -0.72784800 \\
\hline 5 & 0.97326400 & -1.01378700 & 0.38578700 \\
\hline 5 & 2. 27181900 & 1. 28170800 & -0.15191300 \\
\hline 17 & 1. 11413500 & -0.99480200 & 2. 28114900 \\
\hline 6 & 3. 71216200 & -2.33691300 & 1. 52972000 \\
\hline 1 & 3. 73877400 & -1.26661200 & 1. 73018300 \\
\hline 1 & 3. 15079100 & -2.80626300 & 2. 34196100 \\
\hline 1 & 4. 73778800 & -2.71293900 & 1. 57085800 \\
\hline 6 & 0.39970900 & -1.91134100 & -2.42422800 \\
\hline 1 & 0.30465100 & -0.83757000 & -2.26520600 \\
\hline 1 & 0.52298800 & -2.06332800 & -3.49960700 \\
\hline 1 & -0.55656500 & -2.37354200 & -2.14479300 \\
\hline 1 & 1. 98722100 & -3.75126300 & -3.36499100 \\
\hline 1 & 4. 73425400 & -4.03286400 & -0.11549400 \\
\hline 6 & 4. 25423300 & -5.05675700 & -2.58956100 \\
\hline 1 & 4. 95211900 & -4.56259400 & -3.27465500 \\
\hline 1 & 4. 84296600 & -5.68854600 & -1.91952300 \\
\hline 1 & 3. 61036900 & -5.70309300 & -3.19241200 \\
\hline 6 & 7. 79610900 & -0.15769400 & 1. 26396800 \\
\hline 1 & 7. 87449400 & -0.38440300 & 2. 33066000 \\
\hline 1 & 8. 14096000 & -1.02934200 & 0.70217400 \\
\hline 1 & 8. 48598500 & 0.66672400 & 1. 05257000 \\
\hline 6 & 3. 42067300 & 2. 03294000 & 2. 43605400 \\
\hline 1 & 2.54186200 & 1. 44175400 & 2. 71759700 \\
\hline 1 & 3. 98138900 & 2. 25813800 & 3. 34633900 \\
\hline 1 & 3. 05753000 & 2. 98267100 & 2. 02618400 \\
\hline 6 & 4. 07535100 & -0.15995400 & -2.10335100 \\
\hline 1 & 4. 10671300 & -1.24631700 & -2.23713500 \\
\hline 1 & 3. 04758500 & 0.16568600 & -2.28743500 \\
\hline 1 & 4. 71137200 & 0.28915400 & -2.87411900 \\
\hline 1 & 5. 97209400 & 1. 20680500 & 2. 74904200 \\
\hline 1 & 6. 47659900 & -0.69433700 & -1.05685100 \\
\hline 7 & 1. 10588000 & 0.44663100 & -0.12070600 \\
\hline 7 & 1. 70312700 & 2.57069900 & -0.48150300 \\
\hline 7 & 0.37956500 & 2. 45650400 & -0.60840900 \\
\hline 7 & 0.04795900 & 1. 25325300 & -0.38771800 \\
\hline 14 & 2. 31404200 & 4. 26405900 & -0.74823900 \\
\hline 6 & 4. 17385000 & 4. 19568000 & -0.64562400 \\
\hline 1 & 4. 53432300 & 3. 89894700 & 0.34296400 \\
\hline 1 & 4. 59392200 & 3.50089700 & -1.37907800 \\
\hline 1 & 4. 57661500 & 5. 19136700 & -0.86314200 \\
\hline 6 & 1. 51688100 & 5. 28680300 & 0.59491900 \\
\hline 1 & 1. 73373200 & 6.35154900 & 0.46064000 \\
\hline 1 & 0.42937800 & 5. 15826200 & 0.56745000 \\
\hline 1 & 1. 86435500 & 4. 99700500 & 1. 59150400 \\
\hline 6 & 1. 70972100 & 4. 74514800 & -2.44465600 \\
\hline 1 & 2. 00955600 & 5. 76812600 & -2.69382100 \\
\hline 1 & 2. 11690300 & 4. 07738100 & -3.21015900 \\
\hline 1 & 0.61806200 & 4. 69055300 & -2.49541600 \\
\hline 6 & -2.44976100 & 1. 93254900 & 0.15759800 \\
\hline 6 & -2.08560900 & 2. 34496900 & 1. 46634400 \\
\hline 6 & -2.82476800 & 3. 30601500 & 2. 15875900 \\
\hline 6 & -3.96498800 & 3. 88415200 & 1. 61560300 \\
\hline 6 & -4.35151000 & 3. 45229800 & 0.35208900 \\
\hline 6 & -3.63007800 & 2.50632700 & -0.38546500 \\
\hline 6 & -4.26630000 & -1.07873700 & -0.38655600 \\
\hline 6 & -5.07602000 & -0.38161300 & 0.52312900 \\
\hline 6 & -6.37663100 & -0.02699400 & 0.15258800 \\
\hline 6 & -6.90064600 & -0.36915100 & -1.09050100 \\
\hline 6 & -6.09372800 & -1.08693300 & -1.97774100 \\
\hline 6 & -4.78915400 & -1.44232200 & -1.64630900 \\
\hline 5 & -1.48750600 & 0.85829800 & -0.59320700 \\
\hline 5 & -2.78575300 & -1.43764700 & -0.05623100 \\
\hline 17 & -1.62889400 & 0.83934300 & -2.48851600 \\
\hline 6 & -0.91226000 & 1. 75923600 & 2. 21530400 \\
\hline 1 & -0.81333800 & 0.68594700 & 2. 05569700 \\
\hline 1 & 0.04217300 & 2. 22529200 & 1. 93585400 \\
\hline
\end{tabular}

\begin{tabular}{|c|c|c|c|}
\hline 1 & -1.03581400 & 1. 91007100 & 3. 29081900 \\
\hline 6 & -4.22836900 & 2. 17897700 & -1.73660600 \\
\hline 1 & -4.25521700 & 1. 10863000 & -1.93684000 \\
\hline 1 & -5.25381600 & 2. 55543100 & -1.77874400 \\
\hline 1 & -3.66616700 & 2. 64790600 & -2.54847600 \\
\hline 1 & -5.25489900 & 3. 86975200 & -0.08841500 \\
\hline 1 & -2.50362900 & 3. 59496200 & 3. 15775900 \\
\hline 6 & -4.78104800 & 4. 89290300 & 2. 38003100 \\
\hline 1 & -5.62036100 & 4. 41014600 & 2. 89305800 \\
\hline 1 & -4.17875600 & 5. 39898400 & 3. 13889000 \\
\hline 1 & -5.19961100 & 5. 65107000 & 1. 71248600 \\
\hline 6 & -8.31253800 & 0.00392300 & -1.46032500 \\
\hline 1 & -8.58396500 & 0.98049900 & -1.05024300 \\
\hline 1 & -8.44332700 & 0.03944500 & -2.54471200 \\
\hline 1 & -9.02642900 & -0.72689000 & -1.06468200 \\
\hline 6 & -4.58810000 & 0.00002900 & 1. 89864800 \\
\hline 1 & -3.56085900 & -0.32783100 & 2. 08174700 \\
\hline 1 & -4.61692800 & 1. 08635000 & 2. 03328500 \\
\hline 1 & -5.22436600 & -0.44832900 & 2. 66961100 \\
\hline 6 & -3.93627400 & -2.18587400 & -2.64455200 \\
\hline 1 & -4.49824700 & -2.41046800 & -3.55421400 \\
\hline 1 & -3.05828300 & -1.59385500 & -2.92687500 \\
\hline 1 & -3.57193000 & -3.13584700 & -2.23630600 \\
\hline 1 & -6.99124600 & 0.53343400 & 0.85542400 \\
\hline 1 & -6.48812500 & -1.35968300 & -2.95441100 \\
\hline 7 & -1.62000700 & -0.60223200 & -0.08682500 \\
\hline 7 & -2.21679300 & -2.72678100 & 0.27224500 \\
\hline 7 & -0.89328800 & -2.61236300 & 0.39939300 \\
\hline 7 & -0.56198500 & -1.40884500 & 0.17970100 \\
\hline 14 & -2.82719800 & -4.42067900 & 0.53675200 \\
\hline 6 & -4.68708000 & -4.35261100 & 0.43530100 \\
\hline 1 & -5.10693800 & -3.65865700 & 1. 16966800 \\
\hline 1 & -5.04808400 & -4.05491700 & -0.55280000 \\
\hline 1 & -5.08952300 & -5.34860500 & 0.65200400 \\
\hline 6 & -2.22166800 & -4.90423400 & 2. 23203300 \\
\hline 1 & -2.52075100 & -5.92781500 & 2. 47963600 \\
\hline 1 & -1.13001700 & -4.84905700 & 2. 28230500 \\
\hline 1 & -2.62885800 & -4.23799000 & 2. 99885800 \\
\hline 6 & -2.03065900 & -5.44113300 & -0.80853900 \\
\hline 1 & -2.24688800 & -6.50617900 & -0.67562700 \\
\hline 1 & -2.37911700 & -5.15005600 & -1.80440900 \\
\hline 1 & -0.94320000 & -5.31205500 & -0.78175200 \\
\hline \multicolumn{4}{|l|}{64} \\
\hline \multicolumn{4}{|c|}{ TS1_1 } \\
\hline 6 & -1.75049100 & -1.04746300 & 0.61212600 \\
\hline 6 & -2.51424000 & 0.02151800 & 1. 12789300 \\
\hline 6 & -3.91403200 & -0.05161300 & 1. 17884100 \\
\hline 6 & -4.54227400 & -1.19717600 & 0.70535700 \\
\hline 1 & -5.62926900 & -1.25188700 & 0.72468400 \\
\hline 6 & -3.81905900 & -2.28476400 & 0.22343800 \\
\hline 6 & -2.41492000 & -2.22796400 & 0. 19678700 \\
\hline 6 & 2. 41921200 & 0.26453200 & -0.20367800 \\
\hline 6 & 2. 76492900 & 0.65560500 & 1. 13235200 \\
\hline 6 & 4. 04329900 & 0.41956700 & 1. 63729800 \\
\hline 6 & 5. 00209600 & -0.13415300 & 0.78689900 \\
\hline 1 & 5. 99574500 & -0.33546000 & 1. 18362500 \\
\hline 6 & 4. 76321200 & -0.37166400 & -0.56386500 \\
\hline 6 & 3. 47956400 & -0.14360900 & -1.07729900 \\
\hline 5 & -0.17645100 & -0.88315300 & 0.51998100 \\
\hline 5 & 0.83600800 & 0.05457700 & -0.51192400 \\
\hline 35 & 0.81274200 & -2.09783400 & 1. 77334400 \\
\hline 35 & 0.45454200 & -1.45860500 & -1.99882800 \\
\hline 6 & -1.69986900 & -3.46552100 & -0.29076900 \\
\hline 1 & -2.05076000 & -4.34402600 & 0.26216000 \\
\hline 1 & -0.62090600 & -3.41495200 & -0.17735700 \\
\hline 1 & -1.91061100 & -3.65027000 & -1.35092900 \\
\hline 6 & -4.55433000 & -3.51195900 & -0.25158000 \\
\hline 1 & -4.33626600 & -4.38326400 & 0.37617400 \\
\hline
\end{tabular}




\begin{tabular}{|c|c|c|c|}
\hline 1 & -4.27491900 & -3.78264800 & -1.27545200 \\
\hline 1 & -5.63452700 & -3.34899600 & -0.22952000 \\
\hline 6 & -4.73119600 & 1. 09982000 & 1. 70302800 \\
\hline 1 & -4.60272100 & 1. 99319200 & 1. 07942800 \\
\hline 1 & -4.43998900 & 1. 37666600 & 2. 72211200 \\
\hline 1 & -5.79464700 & 0.84883700 & 1. 71412400 \\
\hline 6 & -1.86062000 & 1. 27164800 & 1. 65590900 \\
\hline 1 & -1.90855000 & 1. 31053600 & 2. 75159100 \\
\hline 1 & -2.35121400 & 2. 17355500 & 1. 27417500 \\
\hline 1 & -0.80997400 & 1. 32377700 & 1. 37584000 \\
\hline 6 & 1. 76015900 & 1. 36599400 & 2. 00073900 \\
\hline 1 & 2. 26083100 & 2. 03910800 & 2. 70014700 \\
\hline 1 & 1. 15715400 & 0.66736900 & 2. 59135100 \\
\hline 1 & 1. 08099000 & 1. 97807800 & 1. 40246300 \\
\hline 6 & 4. 40296000 & 0.73753000 & 3. 06622600 \\
\hline 1 & 3. 67413100 & 0.31639900 & 3. 76551100 \\
\hline 1 & 4. 44342200 & 1. 81756900 & 3. 24605100 \\
\hline 1 & 5. 38338700 & 0.32531000 & 3. 31535200 \\
\hline 6 & 5.88701300 & -0.84443300 & -1.44921400 \\
\hline 1 & 6.75436500 & -1.13101900 & -0.85037200 \\
\hline 1 & 6. 20859800 & -0.06037400 & -2.14411600 \\
\hline 1 & 5. 59215100 & -1.70878900 & -2.05173900 \\
\hline 6 & 3. 31220600 & -0.22905100 & -2.57234700 \\
\hline 1 & 3. 30865300 & -1.26357000 & -2.93085800 \\
\hline 1 & 4. 15216500 & 0.28186000 & -3.05502700 \\
\hline 1 & 2. 39208500 & 0.23919600 & -2.91861100 \\
\hline 7 & 0.31524700 & 1. 47425000 & -1.08470400 \\
\hline 7 & 1. 28860900 & 2. 30823700 & -1.26154800 \\
\hline 7 & 2. 42526400 & 2. 33234600 & -1.05941000 \\
\hline 6 & -0.99715400 & 1. 98537300 & -1.35353800 \\
\hline 6 & -1.25329300 & 3. 34890700 & -1.18350000 \\
\hline 6 & -2.01093700 & 1. 11331500 & -1.73391300 \\
\hline 6 & -2.53513200 & 3. 83674600 & -1.39934600 \\
\hline 6 & -3.29788200 & 1. 61309300 & -1.92219800 \\
\hline 6 & -3.56587800 & 2. 96825500 & -1.75880900 \\
\hline 1 & -0.45593400 & 4. 01783700 & -0.87282900 \\
\hline 1 & -1.80548300 & 0.05907600 & -1.86767900 \\
\hline 1 & -2.73133200 & 4. 89614200 & -1.27047500 \\
\hline 1 & -4.09154100 & 0.92719900 & -2.19977100 \\
\hline 1 & -4.57013600 & 3. 34931600 & -1.91267900 \\
\hline \\
\hline \multicolumn{4}{|c|}{ TS1_3 } \\
\hline 6 & 1. 11080000 & -0.51470000 & -0.53822400 \\
\hline 6 & 2. 48899500 & -0.77805300 & -0.74360200 \\
\hline 6 & 3. 43954800 & 0.24328500 & -0.60212700 \\
\hline 6 & 3. 01943100 & 1. 50670600 & -0.19010400 \\
\hline 1 & 3. 76030500 & 2. 29441200 & -0.06157600 \\
\hline 6 & 1. 68923000 & 1. 76914200 & 0.12499400 \\
\hline 6 & 0.72640100 & 0.75730700 & -0.05614300 \\
\hline 6 & -1.48539600 & 1. 96342800 & -2.56588500 \\
\hline 6 & -0.16932000 & 2. 35750200 & -2.84609300 \\
\hline 6 & 0.11006300 & 3. 70426800 & -2.58547200 \\
\hline 6 & -0.90961500 & 4. 52881700 & -2.10534100 \\
\hline 1 & -0.66989300 & 5. 57208400 & -1.91133900 \\
\hline 6 & -2.21430500 & 4. 09596200 & -1.83385600 \\
\hline 6 & -2.53128600 & 2. 75396800 & -2.05026700 \\
\hline 5 & 0.12998500 & -1.73305100 & -0.82369800 \\
\hline 5 & -0.91879000 & -2.56088400 & 0.13655000 \\
\hline 35 & 0. 18690800 & -2.35560300 & -2.79252200 \\
\hline 35 & -0.95347600 & -3.99467900 & 1. 52396800 \\
\hline 6 & -0.69208900 & 1. 05974100 & 0. 35918700 \\
\hline 1 & -1.40978200 & 0.41581500 & -0.14513300 \\
\hline 1 & -0.95760600 & 2. 10627200 & 0.18085600 \\
\hline 1 & -0.81913800 & 0.87611900 & 1. 43384400 \\
\hline 6 & 1. 32008200 & 3. 11706100 & 0.69364500 \\
\hline 1 & 0.86826900 & 3. 01987400 & 1. 68691900 \\
\hline 1 & 0.59624100 & 3. 65159300 & 0. 06770900 \\
\hline 1 & 2. 20701700 & 3. 74843900 & 0.79236700 \\
\hline
\end{tabular}

$\begin{array}{rrr}6 & 4.90445800 & -0.01073000 \\ 1 & 5.07446100 & -0.49745400 \\ 1 & 5.33653500 & -0.66305500 \\ 1 & 5.46591100 & 0.92667300 \\ 6 & 2.98876300 & -2.17020400 \\ 1 & 3.91700600 & -2.37094600 \\ 1 & 3.19494000 & -2.30649000 \\ 1 & 2.27159400 & -2.94018200 \\ 6 & 0.87408900 & 1.42270600 \\ 1 & 0.52362700 & 0.39294400 \\ 1 & 1.74056800 & 1.41061200 \\ 1 & 1.21475000 & 1.76480400 \\ 6 & 1.50463200 & 4.22802500 \\ 1 & 1.84230500 & 4.10138200 \\ 1 & 2.20575900 & 3.68030500 \\ 1 & 1.56151700 & 5.28830600 \\ 6 & -3.23486500 & 5.05824500 \\ 1 & -4.11664200 & 5.11593900 \\ 1 & -2.81130100 & 6.06082900 \\ 1 & -3.57249600 & 4.75392100 \\ 6 & -3.84764700 & 2.12354200 \\ 1 & -3.72368600 & 1.42382000 \\ 1 & -4.26314100 & 1.55674900 \\ 1 & -4.58112100 & 2.87277300 \\ 7 & -1.91739700 & -1.78526700 \\ 7 & -2.11579700 & -0.43826000 \\ 7 & -1.80498100 & 0.62212800 \\ 6 & -3.21040100 & -1.36687400 \\ 6 & -3.58700600 & -0.63252100 \\ 6 & -4.19611100 & -1.58998600 \\ 6 & -4.87879100 & -0.13483100 \\ 6 & -5.48468600 & -1.08887800 \\ 6 & -5.83559500 & -0.34656000 \\ 1 & -2.84575400 & -0.48025000 \\ 1 & -3.92991500 & -2.19093100 \\ 1 & -5.14088300 & 0.42140700 \\ 1 & -6.22586900 & -1.28697400 \\ 1 & -6.84222900 & 0.04246900 \\ 64 & & \end{array}$

$-0.85263700$

$-1.81816800$

$-0.08521400$

$-0.84254400$

$-1.05943200$

$-0.51651200$

$-2.12689400$

$-0.76608300$

$-3.37701400$

$-3.47332500$

$-2.70671300$

$-4.36021500$

$-2.77801100$

$-3.81161400$

$-2.13657800$

$-2.52535400$

$-1.28555500$

$-1.93045800$

$-1.20441900$

-0. 29025000

$-1.69995600$

$-0.86092400$

$-2.53965900$

$-1.40622800$

$-0.32032800$

$-2.79673800$

$-2.72775000$

$-0.13346300$

1. 01166500

$-1.11992700$

1. 14999000

$-0.97077300$

0. 15754000

1. 79003100

$-1.98463300$

2. 04580300

$-1.74038400$

0. 27039200

64

TS1_4

$\begin{array}{llll}6 & 0.94290600 & -2.00855100 & 0.18994700\end{array}$

$\begin{array}{llll}6 & 0.04097900 & -2.11612700 & 1.27078400\end{array}$

$\begin{array}{llll}6 & -0.86423800 & -3.18717600 & 1.33222800\end{array}$

$\begin{array}{llll}6 & -0.82650100 & -4.15343900 & 0.32811700\end{array}$

$\begin{array}{llll}6 & -1.53127700 & -4.98233800 & 0.37087000\end{array}$

$\begin{array}{llll}6 & 0.08829300 & -4.10249700 & -0.72089800\end{array}$

$\begin{array}{llll}6 & 0.98618800 & -3.02363800 & -0.78794000\end{array}$

$\begin{array}{rrrr}6 & -2.70897400 & 0.84741800 & 0.00016500\end{array}$

$\begin{array}{llll}6 & -3.40904000 & -0.19279500 & -0.64621700\end{array}$

$\begin{array}{llll}6 & -4.80578100 & -0.17830800 & -0.53787900\end{array}$

$\begin{array}{llll}6 & -5.42012500 & 0.85225400 & 0.17787900\end{array}$

$\begin{array}{llll}1 & -6.50594100 & 0.85714400 & 0.24327700\end{array}$

$\begin{array}{llll}6 & -4.71643300 & 1.87246600 & 0.81340400\end{array}$

$\begin{array}{llll}6 & -3.31710800 & 1.87286300 & 0.74298300\end{array}$

$\begin{array}{llll}5 & 1.88039800 & -0.73938400 & 0.00309200\end{array}$

$5 \quad 1.98970100 \quad 0.28575500 \quad-1.25230100$

$\begin{array}{llll}35 & 3.57005500 & -0.62489900 & 1.15720500\end{array}$

$\begin{array}{llll}35 & 2.64384900 & 1.22439800 & -2.78031700\end{array}$

$\begin{array}{llll}6 & 1.98196900 & -2.96764500 & -1.92422700\end{array}$

$\begin{array}{llll}6 & 2.26159700 & -3.97060300 & -2.25552500\end{array}$

$1 \quad 2.89378900 \quad-2.44385300 \quad-1.63482900$

$\begin{array}{llll}1 & 1.56933300 & -2.44064300 & -2.79446800\end{array}$

$6 \quad 0.08830600 \quad-5.19577600 \quad-1.76009700$

$1 \quad 1.00810000-5.78982300 \quad-1.72182600$

$\begin{array}{llll}1 & 0.00849200 & -4.79330600 & -2.77525400\end{array}$

$1-0.75073400 \quad-5.87784200 \quad-1.60183800$

$\begin{array}{llll}6 & -1.88243700 & -3.30956600 & 2.43998700\end{array}$

$\begin{array}{llll}1 & -2.49108100 & -2.40353600 & 2.53332600\end{array}$ 


\begin{tabular}{|c|c|c|c|}
\hline 1 & -1.41065000 & -3.48379600 & 3. 41329900 \\
\hline 1 & -2.55793700 & -4.14657500 & 2. 24722500 \\
\hline 6 & 0.05308300 & -1.08123400 & 2. 36863100 \\
\hline 1 & -0.43047700 & -1.44505600 & 3. 27681600 \\
\hline 1 & -0.45951900 & -0.15443900 & 2. 07757300 \\
\hline 1 & 1. 08233600 & -0.81994100 & 2. 62730200 \\
\hline 6 & -2.68613500 & -1.26620300 & -1.41050200 \\
\hline 1 & -1.93802400 & -1.76907800 & -0.78671700 \\
\hline 1 & -2.15012500 & -0.84781500 & -2.26940200 \\
\hline 1 & -3.37814400 & -2.02125200 & -1.78113800 \\
\hline 6 & -5.65871100 & -1.24319500 & -1.18024400 \\
\hline 1 & -5.42296400 & -2.23803500 & -0.78958700 \\
\hline 1 & -5.51750600 & -1.27475400 & -2.26494300 \\
\hline 1 & -6.71592300 & -1.05150400 & -0.98616700 \\
\hline 6 & -5.45096500 & 2.94944400 & 1. 56623400 \\
\hline 1 & -6.52919100 & 2. 85551500 & 1. 42151400 \\
\hline 1 & -5.14902700 & 3. 94756300 & 1. 23360700 \\
\hline 1 & -5.25105800 & 2. 89373800 & 2. 64181100 \\
\hline 6 & -2.51708500 & 2. 95120200 & 1. 42176800 \\
\hline 1 & -1.47756700 & 2. 65829200 & 1. 57235600 \\
\hline 1 & -2.95383700 & 3. 19549700 & 2. 39342200 \\
\hline 1 & -2.52068700 & 3. 87037200 & 0.82445400 \\
\hline 7 & 1. 13307900 & 0.74536600 & -0.15919900 \\
\hline 7 & -0.54617100 & 0.24035500 & -0.64703300 \\
\hline 7 & -1.28904100 & 0.91296100 & -0.03700500 \\
\hline 6 & 1. 09941000 & 2. 00334300 & 0.47576500 \\
\hline 6 & 0.75870500 & 3. 13206600 & -0.28087500 \\
\hline 6 & 1. 31358500 & 2. 12332900 & 1. 85140100 \\
\hline 6 & 0.65438400 & 4. 37383000 & 0. 33441500 \\
\hline 6 & 1. 21404300 & 3. 37207700 & 2. 45728300 \\
\hline 6 & 0.88059400 & 4. 49665100 & 1. 70459700 \\
\hline 1 & 0.57801400 & 3. 02142100 & -1.34580600 \\
\hline 1 & 1. 58527400 & 1. 24597400 & 2. 42467400 \\
\hline 1 & 0.39322500 & 5. 24627700 & -0.25595400 \\
\hline 1 & 1. 39675400 & 3. 46484200 & 3. 52300500 \\
\hline 1 & 0.80045900 & 5. 46725500 & 2. 18361600 \\
\hline \multicolumn{4}{|c|}{64} \\
\hline \multicolumn{4}{|c|}{ TS1_5 } \\
\hline 6 & -0.46761100 & 1. 94585100 & -0.19820300 \\
\hline 6 & -1.77097900 & 1. 62663000 & -0.62242000 \\
\hline 6 & -2.86840800 & 2. 02035300 & 0.16086600 \\
\hline 6 & -2.63267200 & 2. 69174300 & 1. 35985300 \\
\hline 1 & -3.48466500 & 2. 99303800 & 1. 96696200 \\
\hline 6 & -1.34731800 & 2. 99488600 & 1. 81031400 \\
\hline 6 & -0.25204200 & 2. 62175800 & 1. 01824500 \\
\hline 6 & -1.62865600 & -1.90075500 & -0.16782400 \\
\hline 6 & -2.40961600 & -1.43626500 & 0.91932700 \\
\hline 6 & -3.79954000 & -1.60375200 & 0.83708500 \\
\hline 6 & -4.35231900 & -2.25154200 & -0.27014000 \\
\hline 1 & -5.43365100 & -2.36465300 & -0.31910700 \\
\hline 6 & -3.58099400 & -2.76473700 & -1.30785800 \\
\hline 6 & -2.19151000 & -2.58733000 & -1.26392300 \\
\hline 5 & 0.79418100 & 1. 55581800 & -1.03714500 \\
\hline 5 & 2. 01609800 & 0.47475500 & -0.53901200 \\
\hline 35 & 1. 03745400 & 2. 39863200 & -2.78307500 \\
\hline 35 & 3. 91041900 & 0.88315400 & -0.85473200 \\
\hline 6 & 1. 15763600 & 2. 91813500 & 1. 47759100 \\
\hline 1 & 1. 20469400 & 3. 81696200 & 2. 09663800 \\
\hline 1 & 1.84306100 & 3. 08220100 & 0. 63909800 \\
\hline 1 & 1. 56109200 & 2. 08859700 & 2. 07356600 \\
\hline 6 & -1.15823400 & 3. 70458000 & 3. 12698900 \\
\hline 1 & -0.71170400 & 4. 69611000 & 2. 99350700 \\
\hline 1 & -0.50008800 & 3. 14214800 & 3. 79760100 \\
\hline 1 & -2.11665500 & 3. 83909200 & 3. 63386400 \\
\hline 6 & -4.28174000 & 1. 75331900 & -0.29025200 \\
\hline 1 & -4.48226600 & 0.67993400 & -0.38327000 \\
\hline 1 & -4.47588100 & 2. 20031900 & -1.27143600 \\
\hline 1 & -5.00176700 & 2. 17321800 & 0.41674700 \\
\hline
\end{tabular}

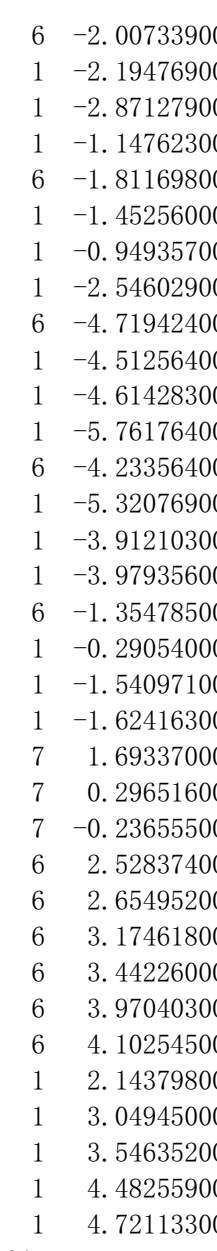

64

\section{TS1_P1}

$6-1.55576300$

$6-1.64820500$

$6-2.89942200$

$6-4.05042900$

$1-5.01565900$

$6 \quad-4.00798400$

$6-2.76193000$

$6-1.34317900$

$6-2.27484500$

$6-3.41898700$

$6-3.54988500$

$1-4.43592100$

$6-2.58031400$

$6-1.45498900$

$5-0.16704100$

$5 \quad 1.40624000$

$35 \quad 2.80929300$

$35 \quad 1.31389400$

$6-2.79095500$

$1-3.36773300$

$1-3.27938900$

$1-1.79633200$

$\begin{array}{ll}6 & -5.28824700\end{array}$

$1-5.43303400$

$1-5.29426500$

$1-6.15123900$

$6-3.00647700$

$1-2.71224800$

$1 \quad-4.03317000$

$1-2.35776400$

$6-0.46497800$

$1-0.69329400$
1. 55894600

0. 20805400

0. 25381600

$-0.80062000$

0. 21184300

$-1.37000300$

$-0.75222300$

$-1.06373000$

$-0.00769600$

$-1.60640400$

$-1.14716400$

$-3.46863200$

$-3.45937800$

$-4.51365500$

$-2.99300100$

$-3.08963200$

$-3.04832700$

$-2.49289100$

$-4.12186000$

$-0.70687400$

$-0.89224700$

$-1.66651000$

$-1.76218800$

$-1.96235600$

$-2.59963700$

$-3.00936300$

$-3.63668400$

$-3.84448400$

$-1.29292800$

$-2.42641200$

$-3.16839800$

$-4.28688700$

$-4.65682000$

$-1.91029800$

$-2.74612700$

$-1.82692100$

$-2.18460800$

2. 14768300

1. 93978000

2. 50271000

2. 95185000

1. 90455000

2. 10867400

2. 85003100

1. 58803400

$-2.46773300$

$-2.36518100$

$-2.53607100$

$-3.42099200$

$-2.41154400$

$-2.19253400$

$-3.31288600$

$-2.65482600$

0. 12246800

0. 47189800

$-0.32402900$

0. 60315500

1. 97722600

$-0.30208700$

2. 44490800

0. 17508800

1. 54593300

2. 66278600

-1. 36609700

3. 51341700

$-0.52687800$

1. 91368000

$-0.98482300$

$-1.75508000$

$-1.98599700$

$-1.52018000$

$-1.67756000$

$-0.93422300$

$-0.69609200$

2. 07623500

2. 34973600

3. 08930300

3. 57152000

4. 14760000

3. 35949000

2. 58702200

$-0.30250300$

$-0.20420600$

$-1.64411200$

0. 30816500

$-0.25414200$

0. 66483300

$-1.03339500$

$-0.10906300$

$-0.56449300$

$-1.14866400$

0. 49182400

$-0.74163800$

$-2.74258000$

$-3.79188800$

$-2.72584100$

$-2.31675500$

$-2.45856200$

$-3.52918900$
0. 40399700

1. 59009600

2. 19286500

1. 56833600

2. 04689600

0. 30502900

$-0.29326900$

$-0.76046100$

0. 24755200

$-0.10126000$

$-1.39960900$

$-1.65715200$

$-2.38111200$

$-2.06416800$

0. 00755800

0. 74536500

0. 40271900

2. 75904000

$-1.73768100$

$-1.88176100$

$-2.33819500$

$-2.15457000$

$-0.39894800$

$-1.31535700$

$-0.69291000$

0. 24730900

3. 49139900

3. 37332900

3. 86498200

4. 26366600

2. 20289500

2. 27167400 


\begin{tabular}{|c|c|c|c|}
\hline 1 & -0.23874100 & -2.11062100 & 3. 21429400 \\
\hline 1 & 0.43655800 & -2.36358400 & 1. 60254700 \\
\hline 6 & -2.09486000 & 1. 90238900 & 1. 67294800 \\
\hline 1 & -2.77206100 & 1. 07736000 & 1. 92272500 \\
\hline 1 & -2.31961200 & 2. 72965600 & 2. 35331100 \\
\hline 1 & -1.07731800 & 1. 57248500 & 1. 88094800 \\
\hline 6 & -4.49074800 & 3. 36156700 & 0.92150700 \\
\hline 1 & -4.13734700 & 4. 04247100 & 1. 70410200 \\
\hline 1 & -4.81030000 & 2. 44069200 & 1. 42084100 \\
\hline 1 & -5.36628200 & 3. 81798800 & 0.45393300 \\
\hline 6 & -2.79140000 & 3. 92494300 & -3.76388300 \\
\hline 1 & -2.83586800 & 3. 13625900 & -4.52228900 \\
\hline 1 & -1.98671800 & 4. 60940400 & -4.05127700 \\
\hline 1 & -3.73045000 & 4. 48095900 & -3.80855100 \\
\hline 6 & -0.43260700 & 2. 24356800 & -3.11622400 \\
\hline 1 & -0.49175100 & 1. 18067400 & -3.38448000 \\
\hline 1 & 0.58622700 & 2. 44782200 & -2.77908900 \\
\hline 1 & -0.59297500 & 2. 81347700 & -4.03110000 \\
\hline 7 & 1. 87337500 & 1. 14520800 & 0.06210500 \\
\hline 7 & 0.91150200 & 1. 83312200 & -0.41901700 \\
\hline 7 & -0.23210200 & 1. 20091200 & -0.44106600 \\
\hline 6 & 3. 16585000 & 1. 75206600 & -0.01313700 \\
\hline 6 & 4. 12446900 & 1. 48046900 & 0.96287500 \\
\hline 6 & 3. 46342700 & 2. 59051800 & -1.08898700 \\
\hline 6 & 5. 37608400 & 2. 07888200 & 0.86567200 \\
\hline 6 & 4. 71739800 & 3. 18405100 & -1.16987200 \\
\hline 6 & 5. 67696800 & 2. 93195000 & -0.19322500 \\
\hline 1 & 3. 88830300 & 0.82477600 & 1. 79138100 \\
\hline 1 & 2. 71725500 & 2. 76831700 & -1.85547200 \\
\hline 1 & 6. 11967400 & 1.87596800 & 1. 62924500 \\
\hline 1 & 4. 94619200 & 3. 83541100 & -2.00694800 \\
\hline 1 & 6. 65718700 & 3. 39232900 & -0.26137500 \\
\hline 7 & 0.33212700 & -1.14241600 & -1.73478400 \\
\hline 7 & 1. 23736700 & -0.51696900 & -2.32584000 \\
\hline 7 & 2. 01783700 & 0.12164300 & -2.82170300 \\
\hline 6 & 0.29732300 & -2.55422000 & -2.04549100 \\
\hline 6 & 1. 41642100 & -3.20607200 & -2.56355400 \\
\hline 6 & -0.89115100 & -3.24219500 & -1.81893700 \\
\hline 6 & 1. 33559500 & -4.56029200 & -2.86492800 \\
\hline 6 & -0.95033500 & -4.60134600 & -2.11384700 \\
\hline 6 & 0.15473800 & -5.26311600 & -2.63961700 \\
\hline 1 & 2. 34908400 & -2.67167200 & -2.71541600 \\
\hline 1 & -1.75157200 & -2.72485000 & -1.41609300 \\
\hline 1 & 2. 20657700 & -5.06630500 & -3.26808600 \\
\hline 1 & -1.87550200 & -5.13906900 & -1.93450400 \\
\hline 1 & 0.09874100 & -6.32148700 & -2.87126300 \\
\hline \multicolumn{4}{|l|}{78} \\
\hline \multicolumn{4}{|c|}{ TS1_P2 } \\
\hline 6 & -1.56084400 & -1.49394100 & 0.28626000 \\
\hline 6 & -1.64367000 & -2.24949700 & 1. 48454100 \\
\hline 6 & -2.87824900 & -2.43640300 & 2. 13289200 \\
\hline 6 & -4.03982600 & -1.94499500 & 1. 54750900 \\
\hline 1 & -4.99201000 & -2.07599700 & 2. 05867300 \\
\hline 6 & -4.01966800 & -1.36135800 & 0.28595800 \\
\hline 6 & -2.78399500 & -1.15902300 & -0.35289700 \\
\hline 6 & -1.16218200 & 1. 58763800 & -0.88342400 \\
\hline 6 & -2.12633600 & 1. 84704000 & 0.10222800 \\
\hline 6 & -3.22568900 & 2. 65211500 & -0.24287000 \\
\hline 6 & -3.29244600 & 3. 21315500 & -1.51455300 \\
\hline 1 & -4.14551200 & 3. 83713700 & -1.77199200 \\
\hline 6 & -2.28409600 & 3. 03019200 & -2.45867300 \\
\hline 6 & -1.19524900 & 2. 20223000 & -2.14724600 \\
\hline 5 & -0.13256400 & -0.95942300 & -0.29756100 \\
\hline 5 & 1. 47956000 & -0.87103900 & 0.56800600 \\
\hline 35 & 2. 92001900 & -2.30189300 & 0.52850000 \\
\hline 35 & 1. 23036000 & -0.23841500 & 2. 54757600 \\
\hline 6 & -2.86525800 & -0.65684600 & -1.77516500 \\
\hline 1 & -3.54500900 & 0.19539200 & -1.87619800 \\
\hline
\end{tabular}

$\begin{array}{ll}1 & -3.23800000 \\ 1 & -1.91333400 \\ 6 & -5.31323200 \\ 1 & -5.47865700 \\ 1 & -5.32645900 \\ 1 & -6.16293500 \\ 6 & -2.96222900 \\ 1 & -2.28327700 \\ 1 & -2.69271600\end{array}$

$1-3.97685900$

$6-0.47592200$

$1-0.74503000$

$1-0.21431300$

$1 \quad 0.42008700$

$\begin{array}{ll}6 & -2.00998000\end{array}$

$1-2.82144200$

$1-2.07128200$

$1-1.06740100$

$6-4.31320000$

$1-3.94355500$

$1-4.70495600$

$1-5.14293600$

$\begin{array}{ll}6 & -2.37417300\end{array}$

$1-2.44182400$

$1-1.49438800$

$1-3.25659800$

$6-0.11813400$

$1 \quad 0.41377300$

$1-0.54808600$

$1 \quad 0.63063300$

$7 \quad 1.99719900$

$7 \quad 1.06083700$

$\begin{array}{ll}7 & -0.10533200\end{array}$

63.30358700

$6 \quad 4.22118800$

63.64812500

$6 \quad 5.48260800$

$6 \quad 4.90897300$

$6 \quad 5.83205100$

$1 \quad 3.94492800$

$1 \quad 2.92527400$

16.19498300

15.17202200

$1 \quad 6.81896700$

$7 \quad 0.45092200$

$7-0.17441900$

$\begin{array}{ll}7 & -0.11720600\end{array}$

$\begin{array}{ll}6 & 0.68485600\end{array}$

$6 \quad 1.82080200$

$\begin{array}{ll}6 & -0.16322200\end{array}$

$\begin{array}{ll}6 & 2.12606600\end{array}$

$\begin{array}{ll}6 & 0.16024900\end{array}$

$6 \quad 1.29826100$

12.46424800

$1-1.05772800$

13.01249000

$1-0.48750100$

$1 \quad 1.54048300$

64

TS1_1a

$6-2.23438900$

$\begin{array}{ll}6 & -2.55990600\end{array}$

$\begin{array}{ll}6 & -3.38165100\end{array}$

$\begin{array}{ll}6 & -3.78964600\end{array}$

$1-4.33142200$

$\begin{array}{ll}6 & -4.06094800\end{array}$

$\begin{array}{ll}6 & -3.31383800\end{array}$

$\begin{array}{ll}6 & 2.02270300\end{array}$
$-1.45259200$

$-0.32092000$

$-0.94951700$

$-1.47819800$

0. 12263100

$-1.16248000$

$-3.17941200$

$-2.75952100$

$-4.23578800$

$-3.13773000$

$-3.01518900$

$-4.07857400$

$-2.69930100$

$-2.94489500$

1. 35057300

0. 66018200

2. 19876800

0. 84159400

2. 92006300

3. 51870200

1. 98909500

3. 46303500

3. 70812600

2. 98258100

4. 32998400

4. 34971500

2. 01761200

2. 96169000

1. 72375100

1. 27895700

0. 43431300

1. 19588500

0.64132400

1. 01265700

0. 78742300

1. 80325500

1. 36878500

2. 38397600

2. 16675300

0. 18158600

1. 95548900

1. 19818100

2. 99840500

2. 61471300

$-1.62589600$

$-1.28814400$

$-0.95570900$

$-3.04690600$

$-3.42871300$

$-4.00268400$

$-4.77434300$

$-5.35040300$

$-5.73520300$

$-2.65819500$

$-3.69309800$

$-5.07392700$

-6. 10020800

-6. 78855700

$-0.51441500$

$-0.27982300$

0.77699200

1. 57270400

2. 49434200

0. 89545900

$-0.21327800$

$-0.43502900$
-2. 43408400

$-2.16415100$

$-0.37045500$

$-1.31635600$

$-0.60193600$

0. 28262400

3. 44145300

4. 19072300

3. 32650700

3. 84525000

2. 05645700

2. 11095100

3. 06975000

1. 44453700

1. 51698600

1. 77119500

2. 20831100

1. 70217500

0. 76394700

1. 60413000

1. 18799200

0. 30521600

$-3.80071000$

$-4.61925900$

$-3.99800300$

$-3.85107200$

$-3.18717500$

$-3.35292200$

$-4.15125100$

$-2.90603200$

$-0.14079100$

$-0.57309200$

$-0.58394000$

$-0.18717200$

0. 83880000

$-1.28490500$

0. 75840200

$-1.34784300$

$-0.32817600$

1. 69251300

$-2.07923800$

1. 55887100

$-2.20278900$

$-0.38159100$

$-1.59457400$

$-2.98307000$

$-4.03895200$

$-1.71302900$

$-2.43250500$

$-1.15630500$

$-2.57269300$

$-1.28739800$

$-1.99122500$

$-2.84672600$

$-0.62980300$

$-3.12134300$

$-0.84549800$

$-2.09164800$

0. 21294100

1. 62945100

1. 90887700

0. 77856200

0. 97725500

$-0.46581100$

$-0.72729600$

0. 10430900 


$\begin{array}{rr}6 & 2.55379500 \\ 6 & 3.92713700 \\ 6 & 4.75726000 \\ 1 & 5.82200300 \\ 6 & 4.26838300 \\ 6 & 2.89612500 \\ 5 & -0.85263100 \\ 5 & 0.46341400 \\ 35 & -0.78650900 \\ 35 & -0.47720500 \\ 6 & -3.60988500 \\ 1 & -3.31958900 \\ 1 & -4.68872700 \\ 1 & -3.09967900 \\ 6 & -5.07606500 \\ 1 & -6.07777000 \\ 1 & -4.84622100 \\ 1 & -5.11950600 \\ 6 & -3.61788400 \\ 1 & -2.69159200 \\ 1 & -3.99410000 \\ 1 & -4.34884800 \\ 6 & -1.84841500 \\ 1 & -2.51613200 \\ 1 & -0.98395600 \\ 1 & -1.50261800 \\ 6 & 1.68533800 \\ 1 & 1.94081800 \\ 1 & 1.80893200 \\ 1 & 0.63044600 \\ 6 & 4.50077700 \\ 1 & 4.08662900 \\ 1 & 4.28253700 \\ 1 & 5.58603400 \\ 6 & 5.21465500 \\ 1 & 5.20209400 \\ 1 & 4.95063800 \\ 1 & 6.23999400 \\ 6 & 2.42812500 \\ 1 & 2.68452100 \\ 1 & 2.91709700 \\ 1 & 1.35223600 \\ 7 & -0.00506600 \\ 7 & -1.34325700 \\ 7 & -2.02153500 \\ 6 & 0.77439300 \\ 6 & 0.52351300 \\ 6 & 1.79694100 \\ 6 & 1.30993300 \\ 6 & 2.58716100 \\ 6 & 2.34615300 \\ 1 & -0.26843600 \\ 1 & 1.97778200 \\ 1 & 1.11999900 \\ & \end{array}$

TS2_1

$\begin{array}{rrrr}7 & 0.74772700 & -1.68160600 & -0.53238900 \\ 7 & 0.11890100 & -2.02293000 & -1.60629900 \\ 7 & -0.88784400 & -1.75182500 & -2.10816600 \\ 6 & 1.95306000 & -2.39409400 & -0.25682000 \\ 6 & 2.87555400 & -1.85243100 & 0.63760300 \\ 6 & 2.20587000 & -3.62148100 & -0.87219300 \\ 6 & 4.04641700 & -2.54890700 & 0.91416600 \\ 6 & 3.38749100 & -4.29960200 & -0.59644600 \\ 6 & 4.31180300 & -3.76959700 & 0.29937300\end{array}$

1. 15079000

1. 20507600

0. 21012800

0. 24618300

$-0.82474700$

$-0.87747400$

$-0.23493700$

0. 06562900

0. 14618900

$-2.33115400$

$-1.92472300$

$-2.86938500$

$-1.95582100$

$-1.85889200$

$-1.41337200$

$-1.19278600$

-2. 45242800

$-1.31856100$

3. 30114900

3. 74879300

3. 95938400

3. 30107900

2. 74372300

3. 60135600

3. 09067500

2. 44424200

2. 27723000

3. 22169600

2. 44006700

2. 07849900

2. 32031600

2. 31845300

3. 30424700

2. 22478800

$-1.86809800$

$-1.90191000$

$-2.87172400$

$-1.66184400$

$-2.00156600$

$-2.97504100$

$-1.94441700$

$-2.00200300$

0. 19042600

0. 22910700

0. 36991900

0. 27143000

$-0.61142300$

1. 21077700

$-0.55038600$

1. 25407300

0. 37743600

$-1.34893100$

1. 88522500

$-1.23905300$

1. 98071900

0. 41606700

3. 7300470

5. 60909100

0. 29937300
$1 \quad 2.68128700$

$-0.89566600$

$-4.04380100$

$-2.12385700$

$-5.25158300$

$-4.30393700$

0. 99889700

2. 02468300

2. 00651100

1. 19381700

2. 99868800

2. 95049300

4. 09859900

4. 87199400

4. 17600600

5. 01533200

3. 16969200

3. 29370200

4. 13539300

2. 38698700

2. 59297400

3. 43234300

1. 78845300

1. 96223000

0. 73451100

0. 13185100

0. 48148700

$-0.29949500$

1. 25839000

$-0.39136800$

$-1.29967100$

$-2.51299600$

$-2.79964700$

$-3.36243300$

$-4.27887800$

$-3.06783600$

$-3.74989100$

$-1.93397900$

$-1.69253500$

$-1.04056200$

0. 09195500

0. 29789500

0. 93864400

2. 03003100

2. 19510300

2. 82695000

3. 65175000

2. 55503500

3. 16745200

1. 53151600

1. 34026400

0. 68913200

$-1.00351500$

$-0.41200700$

$-0.54401600$

$-2.02285100$

64

TS2_3

$\begin{array}{lrrr}7 & 2.14021200 & 0.89993000 & -0.37387200 \\ 7 & 3.60735400 & 0.76262300 & -0.42444800 \\ 7 & 4.14902100 & 0.69278500 & 0.64873100 \\ 6 & 1.64802500 & 1.38918600 & -1.61966800 \\ 6 & 0.62112600 & 0.72344000 & -2.28918200 \\ 6 & 2.20768600 & 2.54579500 & -2.16361200 \\ 6 & 0.13609200 & 1.23627100 & -3.48698200 \\ 6 & 1.72990400 & 3.04154400 & -3.37262000 \\ 6 & 0.68843200 & 2.39358700 & -4.03164700 \\ 1 & 0.20830300 & -0.18692700 & -1.86664300 \\ 1 & 3.01026000 & 3.05232800 & -1.63518400\end{array}$

1. 10777700

$-1.56128200$

1. 61290300

$-1.08165000$

0. 51710100

0. 28608800

1. 15951000

1. 73280600

1. 49189200

2. 55509700

2. 95142300

2. 89653300

3. 56312000

2. 36907900

2. 59878700

1. 47708300

0.90572400

1. 17660800

$-0.05563800$

$-0.71867900$

$-0.39828400$

$-1.74122700$

$-2.24650900$

$-2.17273700$

$-3.03009500$

$-1.52954500$

$-1.91863000$

$-0.40492400$

$-0.45001000$

0.71449800

0. 83175400

1. 18442600

1. 68641000

1. 02206400

1. 41238600

0. 35888900

0. 20006000

$-0.15991900$

$-0.88093600$

$-1.01521500$

$-2.28846300$

$-2.39480000$

$-2.92657600$

$-3.54921000$

$-2.78978500$

$-3.32672800$

$-1.99983900$

$-1.93813900$

$-1.28660000$

1. 89334700

0. 26289500

2. 83607000

2. 33995400

$-1.63518400$
0.04033700

$-1.47746300$ 


\begin{tabular}{|c|c|c|c|}
\hline 1 & -0.67035600 & 0.72298200 & -4.00049300 \\
\hline 1 & 2. 16585700 & 3.94178700 & -3.79364700 \\
\hline 1 & 0.30953500 & 2. 78723100 & -4.96927400 \\
\hline 6 & -0.19486600 & 0.60202900 & 0.75414500 \\
\hline 6 & -0.99982200 & -0.55753600 & 0.78414600 \\
\hline 6 & -0.43320300 & -1.87209500 & 0.69257000 \\
\hline 1 & 0.64497300 & -1.98360500 & 0.60436600 \\
\hline 6 & -1.21979100 & -2.98720200 & 0.70564600 \\
\hline 1 & -0.76646000 & -3.97093800 & 0.63746600 \\
\hline 6 & -2.63705500 & -2.87332500 & 0.80557500 \\
\hline 1 & -3.24644600 & -3.77129600 & 0.81272300 \\
\hline 6 & -3.21987800 & -1.64369900 & 0.88665600 \\
\hline 1 & -4.29968900 & -1.54352900 & 0.95826600 \\
\hline 6 & -2.42890200 & -0.45146800 & 0.87681200 \\
\hline 6 & -3.01555300 & 0.81241100 & 0.94927000 \\
\hline 1 & -4.09765800 & 0.89480400 & 1. 02548700 \\
\hline 6 & -2.24208700 & 1. 97299100 & 0.92524000 \\
\hline 6 & -2.84658000 & 3. 26833900 & 1. 00257900 \\
\hline 1 & -3.92860800 & 3. 32591100 & 1. 08589300 \\
\hline 6 & -2.08747500 & 4. 39906000 & 0.97445100 \\
\hline 1 & -2.55578900 & 5.37630100 & 1. 03530700 \\
\hline 6 & -0.66857100 & 4. 30436500 & 0.86549100 \\
\hline 1 & -0.07467200 & 5. 21280900 & 0.84023300 \\
\hline 6 & -0.05753000 & 3. 08769300 & 0.79368900 \\
\hline 1 & 1. 02348800 & 3. 03001100 & 0.71242800 \\
\hline 6 & -0.81203400 & 1. 86884000 & 0.81919100 \\
\hline 6 & 4. 79853800 & 0.80098700 & 1. 83381100 \\
\hline 6 & 4. 56275100 & 1. 95360600 & 2. 62352900 \\
\hline 6 & 3. 66738400 & 2.97679500 & 2. 20319000 \\
\hline 1 & 3. 16079900 & 2. 86992800 & 1. 24790400 \\
\hline 6 & 3. 45279600 & 4. 07674300 & 2. 98366900 \\
\hline 1 & 2. 75456500 & 4. 84330700 & 2. 66270800 \\
\hline 6 & 4. 13830100 & 4. 22156300 & 4. 22347600 \\
\hline 1 & 3. 96166300 & 5. 10457300 & 4. 82921800 \\
\hline 6 & 5. 00492400 & 3. 25778400 & 4. 65083300 \\
\hline 1 & 5.52874000 & 3. 36356200 & 5. 59675500 \\
\hline 6 & 5. 24444100 & 2. 08074600 & 3. 87422300 \\
\hline 6 & 6.08606700 & 1. 05635600 & 4. 31662100 \\
\hline 1 & 6.57912800 & 1. 14932800 & 5. 28055500 \\
\hline 6 & 6.29619500 & -0.08954200 & 3. 54620400 \\
\hline 6 & 7. 14541100 & -1.15078900 & 3. 99411900 \\
\hline 1 & 7. 61510000 & -1.05375100 & 4. 96893200 \\
\hline 6 & 7. 35941900 & -2.25413900 & 3. 22256700 \\
\hline 1 & 8. 00693700 & -3.04979200 & 3. 57705400 \\
\hline 6 & 6. 73968200 & -2.37405400 & 1. 94411800 \\
\hline 1 & 6.93172100 & -3.25386100 & 1. 33865200 \\
\hline 6 & 5. 91276400 & -1.39169500 & 1. 48137800 \\
\hline 1 & 5. 43105500 & -1.48105600 & 0.51213200 \\
\hline 6 & 5. 66966000 & -0.22606600 & 2. 26397400 \\
\hline 5 & 2. 15501100 & -0.32280600 & 2. 03139500 \\
\hline 5 & 1. 37434000 & 0.44716900 & 0.72714500 \\
\hline 9 & 2. 76518600 & -1.50337100 & 1. 89269200 \\
\hline 9 & 2. 11318300 & 0.15185200 & 3. 26975300 \\
\hline \multicolumn{4}{|c|}{78} \\
\hline \multicolumn{4}{|c|}{ TS2_4 } \\
\hline 7 & -0.04579200 & -0.25016700 & 1. 38453400 \\
\hline 7 & 1. 26996600 & -0.14113900 & 1. 30069000 \\
\hline 7 & 1. 69583400 & -0.22658800 & 0. 10980400 \\
\hline 6 & -0.53915100 & -0.22101500 & 2. 72873400 \\
\hline 6 & -0.00770900 & 0.68214500 & 3. 65062400 \\
\hline 6 & -1.56482800 & -1.08917300 & 3. 09567600 \\
\hline 6 & -0.51743400 & 0.71812800 & 4. 94361300 \\
\hline 6 & -2.07655700 & -1.03207700 & 4. 38825100 \\
\hline 6 & -1.55799700 & -0.13048100 & 5. 31397600 \\
\hline 1 & 0.79749700 & 1. 34544900 & 3. 35127900 \\
\hline 1 & -1.95746700 & -1.79555200 & 2. 37228800 \\
\hline 1 & -0.10515000 & 1. 42135200 & 5. 66002500 \\
\hline 1 & -2.88134800 & -1.70225900 & 4. 67200100 \\
\hline
\end{tabular}

$\begin{array}{rrr}1 & -1.96128600 & -0.09024300 \\ 6 & -2.35951000 & -0.60653600 \\ 6 & -3.26362700 & 0.35009300 \\ 6 & -2.84745600 & 1.47644900 \\ 1 & -1.80471200 & 1.59438100 \\ 6 & -3.73355800 & 2.38706600 \\ 1 & -3.37144900 & 3.20956500 \\ 6 & -5.12883400 & 2.26555100 \\ 1 & -5.81892400 & 3.00386500 \\ 6 & -5.58238200 & 1.21012900 \\ 1 & -6.64316100 & 1.08367300 \\ 6 & -4.68064800 & 0.22664200 \\ 6 & -5.17381100 & -0.85780700 \\ 1 & -6.24496200 & -0.94055000\end{array}$

$6-4.32445100 \quad-1.84522800$

$6-4.85445100 \quad-2.96890300$

$1 \quad-5.93087300 \quad-3.02243800$

$6-4.03495700 \quad-3.94431100$

$1 \quad-4.44395100 \quad-4.79540800$

$6-2.62987500 \quad-3.84691800$

$1-1.97838600 \quad-4.63134600$

$6-2.08819100 \quad-2.78625300$

$1-1.01855300 \quad-2.76203100$

$6-2.90460900 \quad-1.72419200$

$6 \quad 3.11661900 \quad-0.21791200$

$6 \quad 3.67079700 \quad 0.76749300$

$6 \quad 2.87327300 \quad 1.70812600$

$11.79497100 \quad 1.60854000$

$\begin{array}{lll}6 & 3.45906400 & 2.66909500\end{array}$

$1 \quad 2.83807700 \quad 3.36260000$

$6 \quad 4.87891800 \quad 2.76328900$

$1 \quad 5.32315800 \quad 3.53927100$

$6 \quad 5.67042800 \quad 1.87512200$

$16.75292300 \quad 1.92910500$

$65.09849100 \quad 0.84544100$

$6 \quad 5.89396100 \quad-0.07037000$

$1 \quad 6.97612300 \quad-0.00022300$

$6 \quad 5.33519800 \quad-1.08039100$

$6 \quad 6.15646400 \quad-2.03382200$

$17.23496900 \quad-1.94426700$

$6 \quad 5.59991600 \quad-3.03122700$

$16.23032000-3.75194300$

$6 \quad 4.18139500 \quad-3.14739000$

$1 \quad 3.75143600 \quad-3.96704600$

$6 \quad 3.36044500 \quad-2.25306600$

$1 \quad 2.28489100 \quad-2.37270000$

$6 \quad 3.90760900 \quad-1.17400500$

$5 \quad 0.56817000 \quad-0.92641200$

$5-0.77287500 \quad-0.41070700$

$9 \quad 0.75535200 \quad-0.39913400$

$9 \quad 0.97049800 \quad-2.26133900$

$7-0.58139300 \quad 1.45826900$

$7 \quad 0.01997100 \quad 2.22398000$

$7 \quad 0.57657900 \quad 2.79990600$

$\begin{array}{lll}6 & -1.02615400 & 2.00717800\end{array}$

$6-1.63378700 \quad 1.13737300$

$6-0.85912500 \quad 3.35882100$

$6-2.08786400 \quad 1.64192200$

$6-1.31552400 \quad 3.84354700$

$\begin{array}{lll}6 & -1.93381500 & 2.99026100\end{array}$

$1-1.72814900 \quad 0.08518500$

$1-0.38473600 \quad 4.03214100$

$1-2.55971200 \quad 0.96727100$

$1-1.18741800 \quad 4.89527500$

$1-2.29073000 \quad 3.37265500$

78

TS2_5

$7^{-}-0.07110100 \quad-0.17514800$
6. 32048900

0. 00724100

0. 54560300

1. 33454700

1. 59356000

1. 83092300

2. 44005800

1. 57438600

1. 96964900

0. 84510500

0. 64532700

0. 32570700

$-0.39502600$

$-0.56793200$

$-0.88549100$

$-1.59553600$

$-1.73585900$

$-2.07608000$

$-2.61144900$

$-1.86530400$

$-2.23775800$

-1. 19772200

$-1.03924700$

$-0.68029900$

$-0.04102400$

$-0.87384200$

$-1.59969800$

$-1.57373700$

$-2.36921500$

$-2.92788400$

$-2.46470300$

$-3.07980200$

$-1.80034500$

$-1.87505000$

$-0.98770400$

$-0.29837800$

$-0.37991100$

0. 48564600

1. 16721900

1. 07066800

1. 90905700

2. 41991100

2. 00450800

2. 57120100

1. 38417200

1. 44324000

0. 61789000

$-0.94742400$

0. 04458900

$-2.20804400$

$-0.90507200$

$-0.59104700$

0. 17684800

0. 96843900

$-1.84090600$

$-2.74220300$

$-2.13849700$

$-3.95481100$

$-3.35982800$

-4. 26880700

$-2.50375100$

$-1.42978300$

$-4.66123800$

$-3.59396400$

$-5.21912400$ 


\begin{tabular}{|c|c|c|c|}
\hline 7 & 1. 22827200 & -0.11614400 & 1. 35362500 \\
\hline 7 & 1. 64443300 & -0.15500800 & 0. 14698800 \\
\hline 6 & -0.56541600 & -0.18509200 & 2. 79572400 \\
\hline 6 & 0.06820300 & 0.58285100 & 3. 77568100 \\
\hline 6 & -1.67275400 & -0.96910900 & 3. 11593700 \\
\hline 6 & -0.42308500 & 0.57185300 & 5. 07608500 \\
\hline 6 & -2.15980700 & -0.96061700 & 4. 41939700 \\
\hline 6 & -1.54208300 & -0.19100600 & 5. 40080800 \\
\hline 1 & 0.93808300 & 1. 17516600 & 3. 51545300 \\
\hline 1 & -2.13818100 & -1.58584000 & 2. 35702800 \\
\hline 1 & 0.06896400 & 1. 17097300 & 5. 83541700 \\
\hline 1 & -3.02406900 & -1.56843300 & 4. 66593200 \\
\hline 1 & -1.92741800 & -0.18868100 & 6. 41507900 \\
\hline 6 & -2.44900900 & -0.45590800 & 0. 12178800 \\
\hline 6 & -3.44924800 & 0.51389900 & 0.38173200 \\
\hline 6 & -3.17457000 & 1. 72750400 & 1. 09608000 \\
\hline 1 & -2.20557300 & 1. 85405700 & 1. 56387400 \\
\hline 6 & -4.13120500 & 2. 67859300 & 1. 30036500 \\
\hline 1 & -3.89220500 & 3.57167900 & 1. 86926300 \\
\hline 6 & -5.45430300 & 2. 49611700 & 0.80291000 \\
\hline 1 & -6.19959700 & 3. 27000300 & 0.95580200 \\
\hline 6 & -5.78373000 & 1. 33246500 & 0.17631300 \\
\hline 1 & -6.79871500 & 1. 15380900 & -0.16919600 \\
\hline 6 & -4.81291300 & 0.29926600 & -0.02294900 \\
\hline 6 & -5.17639400 & -0.92274400 & -0.58369300 \\
\hline 1 & -6.20396100 & -1.08210400 & -0.90359700 \\
\hline 6 & -4.25162300 & -1.95974400 & -0.69746900 \\
\hline 6 & -4.64886100 & -3.24850600 & -1.17522700 \\
\hline 1 & -5.68846100 & -3.39171000 & -1.45811300 \\
\hline 6 & -3.75475100 & -4.27353200 & -1.25638400 \\
\hline 1 & -4.06874100 & -5.25023900 & -1.61082700 \\
\hline 6 & -2.40282000 & -4.06634100 & -0.85878900 \\
\hline 1 & -1.70045200 & -4.89287800 & -0.90114900 \\
\hline 6 & -1.98279000 & -2.84237200 & -0.42368800 \\
\hline 1 & -0.95498700 & -2.70885500 & -0.11484900 \\
\hline 6 & -2.87832800 & -1.72785900 & -0.33377600 \\
\hline 6 & 3. 06549000 & -0.19851700 & -0.02516200 \\
\hline 6 & 3. 66510900 & 0.87336700 & -0.70220500 \\
\hline 6 & 2. 91483000 & 1. 97685600 & -1.21863000 \\
\hline 1 & 1. 83451700 & 1. 96447800 & -1.12334500 \\
\hline 6 & 3.54591400 & 3. 00636200 & -1.85115400 \\
\hline 1 & 2. 96372300 & 3. 83200300 & -2.24852300 \\
\hline 6 & 4. 96389800 & 3. 00935600 & -2.00775000 \\
\hline 1 & 5. 44328300 & 3. 84135100 & -2.51329300 \\
\hline 6 & 5. 70993600 & 1. 97339600 & -1.53087400 \\
\hline 1 & 6. 78998200 & 1. 96353200 & -1.64769300 \\
\hline 6 & 5. 09005300 & 0.86959700 & -0.86408500 \\
\hline 6 & 5.83493900 & -0.19744200 & -0.35997400 \\
\hline 1 & 6.91593000 & -0.19295800 & -0.47920500 \\
\hline 6 & 5. 22739700 & -1.27465600 & 0.28707800 \\
\hline 6 & 5. 99449100 & -2.37447100 & 0.78645200 \\
\hline 1 & 7. 07307300 & -2.34806300 & 0.65889100 \\
\hline 6 & 5. 38732600 & -3.42891200 & 1. 39858600 \\
\hline 1 & 5. 97737200 & -4.26027800 & 1. 77068800 \\
\hline 6 & 3.96853200 & -3.45448300 & 1. 54396300 \\
\hline 1 & 3. 49805400 & -4.31099400 & 2. 01588500 \\
\hline 6 & 3. 19810800 & -2.42427100 & 1. 09203500 \\
\hline 1 & 2. 11970300 & -2.46602000 & 1. 19472700 \\
\hline 6 & 3. 80129500 & -1.29112800 & 0.45809600 \\
\hline 5 & 0.59201000 & -0.64287400 & -0.97587000 \\
\hline 5 & -0.89297900 & -0.03435900 & 0.14569100 \\
\hline 9 & 0.83380500 & -0.03386200 & -2.17070500 \\
\hline 9 & 0.73857400 & -2.00832600 & -1.06167700 \\
\hline 7 & -0.56297200 & 1. 34766600 & -0.43684100 \\
\hline 7 & -0.26345300 & 2. 40762100 & 0.51212800 \\
\hline 7 & 0.42736300 & 2. 62316600 & 1. 37949900 \\
\hline 6 & -1.12497300 & 1.89142500 & -1.63729600 \\
\hline 6 & -1.73370500 & 1. 03593600 & -2.55903000 \\
\hline
\end{tabular}

$\begin{array}{rrr}6 & -1.01512600 & 3.25456100 \\ 6 & -2.22124100 & 1.55063500 \\ 6 & -1.51277500 & 3.75444000 \\ 6 & -2.11873000 & 2.90736700 \\ 1 & -1.80945500 & -0.02459600 \\ 1 & -0.56624400 & 3.93519100 \\ 1 & -2.68595700 & 0.87300100 \\ 1 & -1.42798900 & 4.81692800 \\ 1 & -2.50877200 & 3.30064600\end{array}$

$-1.92501200$

$-3.75442100$

$-3.12208200$

$-4.04470600$

$-2.35592900$

$-1.20860300$

$-4.46295500$

-3. 32587500

$-4.97740300$

TS2_1a

$7 \quad 2.03209200$

$7 \quad 2.18924600$

73.03219800

63.17502200

63.28120200

64.14458000

64.36051500

$6 \quad 5.22247600$

$6 \quad 5.33490000$

12.52899200

14.05828000

14.44347300

15.97502400

16.17767300

$6 \quad 0.13074600$

60.77629300

$6 \quad 0.51640100$

$1-0.21194600$

61.19674400

10.97522600

$6 \quad 2.19391100$

12.72438900

$6 \quad 2.51629300$

13.31269500

$6 \quad 1.80199700$

62.19524800

13.06118500

$6 \quad 1.20381200$

$6 \quad 1.32389200$

12.15668200

$6 \quad 0.37813600$

10.45294900

$6-0.68879100$

$1-1.43252200$

$6-0.79511600$

$1-1.61859600$

$6 \quad 0.14387400$

$6-2.07803700$

$\begin{array}{ll}6 & -2.41898000\end{array}$

$6-1.44577200$

$1-0.41177700$

$6-1.79256900$

$1-1.03268100$

$6-3.14405000$

$1-3.39961700$

$6-4.10817600$

$1-5.14620000$

$\begin{array}{ll}6 & -3.78301500\end{array}$

$6-4.77393400$

$1-5.80344700$

$6-4.47757800$

$6-5.50982700$

$1-6.52410100$

$6-5.23178000$

$1-6.02122900$

$6-3.89648200$

$1-3.68443500$
0. 82108300

$-0.25821600$

$-0.83645600$

1. 65593400

2. 33070800

1. 82276800

3. 17782700

2. 67119800

3. 35209400

2. 17908900

1. 30404700

2. 80011400

4. 01169400

$-2.27562600$

$-2.50987700$

$-1.89748900$

$-3.49954600$

$-3.68789600$

$-4.25374800$

$-5.02695800$

$-3.99343500$

$-4.53962700$

$-3.01418400$

$-2.58365200$

$-3.05780600$

$-2.01980600$

$-2.04565600$

$-2.57689200$

$-1.46277500$

$-0.72360900$

$-0.23857400$

$-0.64759200$

$-0.10464800$

$-1.30959100$

0. 42054100

1. 72129700

2. 60356100

2. 27982500

3. 83420800

4. 48227400

4. 28159800

5. 26560200

3. 47123400

3. 79068300

2. 16955500

1. 32002600

1. 66979400

0. 02355900

$-0.85055000$

$-0.46366100$

$-2.13238500$

$-2.78850700$

$-2.62096500$

$-3.65237100$
3. 69752200

$-1.21610700$

$-1.42030400$
$-0.37286900$

0. 33216700

0. 91544100

$-0.57199600$

$-1.79042900$

0. 41737600

$-2.00725200$

0. 18177900

$-1.02635200$

$-2.55466400$

1. 36603200

$-2.95644200$

0. 95312700

-1. 20544200

0. 28709700

$-0.46613400$

$-1.83605800$

$-2.35799600$

$-2.51095100$

$-3.55621200$

$-1.86218600$

$-2.40844900$

$-0.54899000$

$-0.05219000$

0. 16970800

1. 48099200

1. 93581300

2. 34991200

3. 75294300

4. 20429600

4. 53445000

5. 61633500

3. 93422700

4. 55783700

2. 56301500

2. 11365400

1. 73589900

$-0.28587100$

0. 17051400

0. 74291300

0. 80081500

1. 21928800

1. 16507200

1. 54529600

0. 64523900

0. 60913200

0. 14901600

$-0.34350000$

$-0.38355000$

$-0.76016100$

$-1.22847000$

$-1.27926000$

$-1.59526400$

$-1.94754200$

$-1.50151400$

$-1.76537200$
1. 64413600 


$\begin{array}{rr}6 & -2.88526800 \\ 1 & -1.88192200 \\ 6 & -3.11951900 \\ 5 & 0.65044400 \\ 5 & -0.56602500 \\ 9 & 0.44099600 \\ 9 & 0.85035400\end{array}$

\section{TS3_1}

$6 \quad 2.26324900$

$\begin{array}{ll}6 & 3.37926100\end{array}$

$6 \quad 3.63827200$

13.02705000

$6 \quad 4.68787200$

14.88946300

$6 \quad 5.51323500$

$1 \quad 6.32077300$

$6 \quad 5.31552300$

$1 \quad 5.96737800$

$\begin{array}{ll}6 & 4.28401100\end{array}$

64.14458700

14.77106200

$\begin{array}{ll}6 & 3.38160900\end{array}$

6
6 3.46003900

14.16437800

$6 \quad 2.68121100$

$\begin{array}{ll}1 & 2.75730000\end{array}$

$6 \quad 1.79034900$

$1 \quad 1.20635500$

$6 \quad 1.66518300$

$1 \quad 1.02476300$

$\begin{array}{ll}6 & 2.44323600\end{array}$

$\begin{array}{ll}6 & -1.99944900\end{array}$

$\begin{array}{ll}6 & -2.81647000\end{array}$

$\begin{array}{ll}6 & -2.31016500\end{array}$

$\begin{array}{ll}1 & -1.25371600\end{array}$

$\begin{array}{ll}6 & -3.12079800\end{array}$

$\begin{array}{ll}1 & -2.70281700\end{array}$

$\begin{array}{ll}6 & -4.51015100\end{array}$

$1-5.13732200$

$\begin{array}{ll}6 & -5.04068300\end{array}$

$\begin{array}{ll}1 & -6.09784100\end{array}$

$\begin{array}{ll}6 & -4.22489000\end{array}$

$\begin{array}{ll}6 & -4.78951600\end{array}$

$1-5.85429000$

$\begin{array}{ll}6 & -4.02035900\end{array}$

$6-4.60950000$

$\begin{array}{ll}1 & -5.67306500\end{array}$

$\begin{array}{ll}6 & -3.86297400\end{array}$

$\begin{array}{lll}1 & -4.32071900\end{array}$

$\begin{array}{ll}6 & -2.47310300\end{array}$

$1-1.88662900$

$\begin{array}{ll}6 & -1.87926100\end{array}$

$\begin{array}{ll}1 & -0.82655500\end{array}$

$\begin{array}{ll}6 & -2.61357000\end{array}$

$\begin{array}{ll}5 & 0.86707700\end{array}$

$\begin{array}{ll}5 & -0.43059700\end{array}$

$\begin{array}{ll}35 & 0.66406700\end{array}$

$35 \quad 0.51507600$

$\begin{array}{ll}7 & -0.00983000\end{array}$

$7 \quad 1.31392700$

$7 \quad 2.00677500$

$\begin{array}{ll}6 & -0.84197600\end{array}$

$6-1.71628400$

$\begin{array}{ll}6 & -0.76093100\end{array}$

$\begin{array}{ll}6 & -2.54554700\end{array}$

$\begin{array}{ll}6 & -1.58296300\end{array}$

$\begin{array}{lr}6 & -2.47836800\end{array}$

$\begin{array}{rr}-1.80878100 & -1.07778500 \\ -2.20961100 & -1.00125600 \\ -0.44730000 & -0.70040100 \\ 0.96817200 & -1.20831400 \\ 0.02388800 & -0.36376200 \\ 2.32903100 & -1.36449300 \\ 0.36881700 & -2.46413500\end{array}$

0. 26171700

0. 48533600

$-0.01654600$

1. 27485600

1. 40096700

1. 92248800

2. 56465600

1. 70618700

2. 15693200

0. 83314500

0. 49595800

0. 99439600

$-0.61959400$

$-1.14520000$

$-0.69941900$

$-2.20893400$

$-2.62351400$

$-2.78326400$

$-3.65447100$

-2. 26613700

$-2.75335200$

$-1.15604200$

$-0.21076900$

0. 83646100

2. 14444100

2. 34238900

3. 13988900

4. 11912000

2. 90941400

3. 71050900

1. 68521600

1. 48753000

0. 62284400

$-0.61345100$

$-0.76894100$

$-1.64228000$

$-2.89084300$

$-3.02974900$

$-3.87353700$

$-4.81430800$

$-3.66315600$

$-4.44415300$

$-2.49209900$

$-2.33349300$

$-1.43409400$

$-0.71560500$

$-0.05285000$

$-2.54039400$

0. 78610800

0.93088800

1. 15874100

1. 83154400

1. 69008900

0. 99351000

3. 07854400

1. 71081800

3. 78255200

3. 10070100
0. 22253300

0. 69243300

2. 07085900

2. 80834900

2. 47143100

3. 53022300

1. 52269600

1. 85922900

0. 18447100

$-0.55819100$

$-0.25025400$

$-1.61362100$

$-2.35048400$

$-2.00074000$

$-3.31868300$

$-4.01522500$

$-3.69016500$

$-4.68977100$

$-2.75307600$

$-3.03365600$

$-1.48588900$

$-0.76196300$

$-1.06746000$

0. 77696900

1. 07823600

0. 94470500

1. 53982200

1. 75208700

1. 75528100

2. 13346800

1. 48371300

1. 63937500

0. 97917300

0. 66952800

0. 82970900

0. 13186700

$-0.24627000$

$-0.07114400$

$-0.82393900$

$-1.11298500$

$-1.06060000$

$-1.53503800$

$-0.69187900$

$-0.88964800$

$-0.06709800$

1. 01175400

0. 11783300

1. 90175400

2. 49269900

$-0.89625500$

$-1.09202900$

$-1.69935400$

$-1.80663600$

$-2.63280900$

$-1.83568400$

$-3.49026800$

$-2.70907200$

$-3.53042200$
0.28391900 $\begin{array}{ll}1 & -1.74706900 \\ 1 & -0.06899300 \\ 1 & -3.23797100 \\ 1 & -1.53072700 \\ 1 & -3.12336300 \\ 4 & \end{array}$

\section{TS3_1a}

$6 \quad 0.34582300$

$6-0.91494200$

$6-1.84558500$

$1-1.54664200$

$\begin{array}{ll}6 & -3.07302700\end{array}$

$\begin{array}{ll}6 & -3.75109900\end{array}$

$6-3.46436300$

$1-4.44623700$

$\begin{array}{ll}6 & -2.59662800\end{array}$

$1-2.87032400$

$\begin{array}{ll}6 & -1.29596300\end{array}$

$6-0.38832000$

$1-0.67759900$

$6 \quad 0.89267500$

$6 \quad 1.83989200$

$1 \quad 1.53215100$

63.09464200

13.80725700

$6 \quad 3.47886800$

14.48256800

$6 \quad 2.59814400$

$1 \quad 2.91409200$

$6 \quad 1.26849000$

$\begin{array}{ll}6 & -1.32763000\end{array}$

$\begin{array}{ll}6 & -2.35383000\end{array}$

$6-2.08777900$

$1-1.07999500$

$\begin{array}{ll}6 & -3.08798700\end{array}$

$1-2.84369500$

$6-4.43824600$

$1-5.20994500$

$\begin{array}{ll}6 & -4.75302400\end{array}$

$1-5.78227400$

$\begin{array}{ll}6 & -3.73669000\end{array}$

$\begin{array}{ll}6 & -4.09506700\end{array}$

$1-5.14271900$

$\begin{array}{ll}6 & -3.15590100\end{array}$

$6-3.56817200$

$1-4.62709100$

$\begin{array}{ll}6 & -2.65613200\end{array}$

$1-2.96843000$

$6-1.28630700$

$1-0.56116700$

$\begin{array}{ll}6 & -0.86063500\end{array}$

10.19424500

$\begin{array}{ll}6 & -1.76965000\end{array}$

$5 \quad 0.71566400$

$5 \quad 0.22138300$

$35 \quad 1.90458300$

$35 \quad 0.76792200$

$7 \quad 1.11401300$

$7 \quad 0.40871100$

$7-0.70638300$

6
6

$6 \quad 3.45974000$

$\begin{array}{ll}6 & 2.88618600\end{array}$

$6 \quad 4.80178300$

64.23095300

$6 \quad 5.19212000$

13.15374700

$1 \quad 2.12767900$

$-0.09085400$

3. 59933900

1. 17720100

4. 86568100

3. 65539200

1. 88621700

2. 33766700

1. 46924800

0. 45002700

1. 90767500

1. 22488400

3. 26150900

3. 59459800

4. 13524200

5. 17673000

3. 71348100

4. 61376000

5. 65464500

4. 20793600

5. 14568900

6. 18324200

4. 75160200

5. 47119200

3. 38623900

3. 08278000

2. 46368500

1. 42983700

2. 82877000

$-1.33368800$

$-1.14730000$

$-1.10529300$

$-1.27047800$

$-0.91553700$

$-0.90936200$

$-0.74555400$

$-0.57919400$

$-0.84040900$

$-0.76674900$

$-1.07977200$

$-1.30604100$

$-1.23165700$

$-1.69335100$

$-2.01519700$

$-1.95625600$ 


\begin{tabular}{|c|c|c|c|}
\hline 1 & 5.54529200 & -1.00856600 & -0.86832400 \\
\hline 1 & 4. 52420800 & -4.66315100 & 1. 14409000 \\
\hline 1 & 6. 24067600 & -3.03155400 & 0.39194100 \\
\hline \multicolumn{4}{|l|}{64} \\
\hline \multicolumn{4}{|c|}{ TS3_1b } \\
\hline 6 & 1. 95210000 & -0.07966800 & -0.79636700 \\
\hline 6 & 3. 00114300 & -1.03102200 & -0.89445300 \\
\hline 6 & 2. 80989400 & -2.34750500 & -1.43518800 \\
\hline 1 & 1. 84775800 & -2.60419600 & -1.85856700 \\
\hline 6 & 3. 82726500 & -3.25523000 & -1.49180000 \\
\hline 1 & 3. 65204600 & -4.23192800 & -1.93216300 \\
\hline 6 & 5. 12519800 & -2.93213100 & -1.00172100 \\
\hline 1 & 5.91624900 & -3.67487300 & -1.03962400 \\
\hline 6 & 5. 37277400 & -1.68011600 & -0.52755300 \\
\hline 1 & 6.36621000 & -1.39613600 & -0.19005100 \\
\hline 6 & 4. 34037400 & -0.68824500 & -0.49191100 \\
\hline 6 & 4. 63096300 & 0.61539100 & -0.09360400 \\
\hline 1 & 5. 63899200 & 0.86636700 & 0.23059500 \\
\hline 6 & 3. 66489400 & 1. 61590500 & -0.17601000 \\
\hline 6 & 3. 99421200 & 2. 97839700 & 0.11127800 \\
\hline 1 & 5. 01486100 & 3. 20483200 & 0.41025200 \\
\hline 6 & 3. 06829000 & 3.97138600 & -0.01703200 \\
\hline 1 & 3. 33509600 & 5. 00455900 & 0.18292500 \\
\hline 6 & 1. 74764400 & 3. 64935700 & -0.43946000 \\
\hline 1 & 1. 01914600 & 4. 44287800 & -0.57721300 \\
\hline 6 & 1. 39261700 & 2. 35266400 & -0.68102900 \\
\hline 1 & 0.38636700 & 2. 13644600 & -1.01494900 \\
\hline 6 & 2.32171100 & 1. 26898600 & -0.55454000 \\
\hline 6 & -2.42808100 & 0.05624100 & 0.07500600 \\
\hline 6 & -3.19824400 & -0.73518700 & 0.97201600 \\
\hline 6 & -2.62220200 & -1.52281200 & 2. 02803300 \\
\hline 1 & -1.54572900 & -1.54036800 & 2. 15138700 \\
\hline 6 & -3.39167100 & -2.25957000 & 2. 87988200 \\
\hline 1 & -2.91658200 & -2.83687700 & 3. 66693300 \\
\hline 6 & -4.80971600 & -2.29041000 & 2. 74685700 \\
\hline 1 & -5.40289500 & -2.89546800 & 3. 42468400 \\
\hline 6 & -5.40671800 & -1.55414800 & 1. 77039100 \\
\hline 1 & -6.48676700 & -1.55437800 & 1. 65142400 \\
\hline 6 & -4.63313400 & -0.74792200 & 0.87481000 \\
\hline 6 & -5.26568700 & 0.04077500 & -0.08319400 \\
\hline 1 & -6.35139300 & 0.02608600 & -0.15123500 \\
\hline 6 & -4.54029800 & 0.86870500 & -0.93655400 \\
\hline 6 & -5.20970700 & 1. 71799700 & -1.87308600 \\
\hline 1 & -6.29492600 & 1. 68057000 & -1.91241900 \\
\hline 6 & -4.50662800 & 2.55538800 & -2.68537500 \\
\hline 1 & -5.02058000 & 3. 19931500 & -3.39179600 \\
\hline 6 & -3.08451600 & 2.58433700 & -2.60809100 \\
\hline 1 & -2.52594400 & 3. 24811500 & -3.26034600 \\
\hline 6 & -2.41689700 & 1. 77701600 & -1.73443600 \\
\hline 1 & -1.33587000 & 1. 79482900 & -1.71888400 \\
\hline 6 & -3.10512000 & 0.87760300 & -0.85829800 \\
\hline 5 & 0.39302600 & -0.45744000 & -0.92780000 \\
\hline 5 & -0.84585200 & 0.08378700 & 0. 18033500 \\
\hline 35 & -0.19366800 & -0.51295300 & -2.88274800 \\
\hline 35 & -0.10958100 & -2.38090200 & -0.05210400 \\
\hline 7 & -0.33637800 & 0.71724800 & 1. 38026900 \\
\hline 7 & -1.47900500 & 1. 13492200 & 2. 36941100 \\
\hline 7 & -2.05514200 & 1. 84123200 & 2. 99481200 \\
\hline 6 & 0.88099400 & 0.79548500 & 2. 10780400 \\
\hline 6 & 1. 64339200 & -0.35389900 & 2. 31773400 \\
\hline 6 & 1. 32967800 & 2. 05652400 & 2. 51407100 \\
\hline 6 & 2. 90065200 & -0.22381600 & 2. 89959800 \\
\hline 6 & 2.57110900 & 2. 16736200 & 3. 12089500 \\
\hline 6 & 3. 35836900 & 1. 02815800 & 3. 30245800 \\
\hline 1 & 1. 27308700 & -1.31752900 & 1. 98675300 \\
\hline 1 & 0.71937300 & 2. 93319400 & 2. 31827000 \\
\hline 1 & 3. 51977500 & -1.10402800 & 3. 03489200 \\
\hline 1 & 2. 93620900 & 3. 14061000 & 3. 42969800 \\
\hline
\end{tabular}

64

TS3_1c

$\begin{array}{lllr}6 & 2.25174600 & -0.20018300 & -0.53704800 \\ 6 & 3.43482000 & -0.74866200 & 0.07043200 \\ 6 & 3.69459100 & -2.14528100 & 0.11647800\end{array}$

$13.01243300 \quad-2.82170100 \quad-0.37930200$

$\begin{array}{llll}6 & 4.82370300 & -2.63947300 & 0.72134700\end{array}$

$\begin{array}{llll}1 & 5.01160300 & -3.70821700 & 0.70950200\end{array}$

$\begin{array}{llll}6 & 5.75146900 & -1.77568800 & 1.35105100\end{array}$

$\begin{array}{llll}1 & 6.62488600 & -2.18877200 & 1.84515500\end{array}$

$\begin{array}{llll}6 & 5.56223700 & -0.42178000 & 1.29408700\end{array}$

$\begin{array}{llll}1 & 6.28565500 & 0.26403700 & 1.72559500\end{array}$

$\begin{array}{llll}6 & 4.43461800 & 0.11916700 & 0.61587900\end{array}$

$\begin{array}{llll}6 & 4.31857400 & 1.50754700 & 0.43208000\end{array}$

$\begin{array}{llll}1 & 5.04520100 & 2.16900000 & 0.89955000\end{array}$

$\begin{array}{llll}6 & 3.37060500 & 2.03809200 & -0.44628900\end{array}$

$\begin{array}{llll}6 & 3.36855300 & 3.42678000 & -0.77104300\end{array}$

$14.13145700 \quad 4.06087200 \quad-0.32790500$

$\begin{array}{llll}6 & 2.44190500 & 3.94200600 & -1.63424100\end{array}$

$\begin{array}{llll}1 & 2.45093700 & 4.99630500 & -1.88939100\end{array}$

$\begin{array}{llll}6 & 1.48814500 & 3.07724700 & -2.22765700\end{array}$

$\begin{array}{llll}1 & 0.79339000 & 3.47001700 & -2.96440900\end{array}$

$\begin{array}{llll}6 & 1.44353200 & 1.74387800 & -1.90162400\end{array}$

$\begin{array}{llll}1 & 0.76394900 & 1.08188500 & -2.41979200\end{array}$

$\begin{array}{llll}6 & 2.35814800 & 1.17423600 & -0.97761400\end{array}$

$\begin{array}{llll}6 & -2.05719700 & -0.33864700 & -0.14385800\end{array}$

$\begin{array}{llll}6 & -2.80855400 & -0.97107100 & 0.87804800\end{array}$

$\begin{array}{llll}6 & -2.24352200 & -1.37765200 & 2.13353300\end{array}$

$\begin{array}{llll}1 & -1.18869300 & -1.21211300 & 2.31154900\end{array}$

$\begin{array}{llll}6 & -2.99698100 & -1.97964700 & 3.09865300\end{array}$

$\begin{array}{llll}1 & -2.53485300 & -2.27347200 & 4.03618000\end{array}$

$\begin{array}{llll}6 & -4.38159200 & -2.24110900 & 2.88936700\end{array}$

$\begin{array}{llll}1 & -4.96073600 & -2.73171300 & 3.66526800\end{array}$

$6-4.96597300 \quad-1.87501100 \quad 1.71561400$

$\begin{array}{llll}1 & -6.02050800 & -2.06406800 & 1.53360200\end{array}$

$\begin{array}{llll}6 & -4.21293700 & -1.22373400 & 0.68661600\end{array}$

$6-4.83608200 \quad-0.81938700 \quad-0.49241600$

$\begin{array}{llll}6 & -5.89652200 & -1.01844200 & -0.63105200\end{array}$

$\begin{array}{llll}6 & -4.13160100 & -0.13994200 & -1.48327900\end{array}$

$\begin{array}{llll}6 & -4.78294400 & 0.32501600 & -2.66998600\end{array}$

$1-5.84014200 \quad 0.10553400 \quad-2.79304300$

$\begin{array}{llll}6 & -4.10230300 & 1.03150600 & -3.61562100\end{array}$

$\begin{array}{llll}1 & -4.60718000 & 1.38258200 & -4.50996300\end{array}$

$\begin{array}{llll}6 & -2.71962400 & 1.31919300 & -3.42673700\end{array}$

$\begin{array}{llll}1 & -2.18365600 & 1.89149600 & -4.17762600\end{array}$

$\begin{array}{llll}6 & -2.06868600 & 0.87397300 & -2.31423600\end{array}$

$\begin{array}{llll}1 & -1.02342700 & 1.10879900 & -2.16853800\end{array}$

$\begin{array}{llll}6 & -2.73016400 & 0.11448100 & -1.29949500\end{array}$

$\begin{array}{llll}5 & 0.84666900 & -0.97752100 & -0.72553700\end{array}$

$\begin{array}{rrrr}5 & -0.47995500 & -0.12888600 & 0.01777300\end{array}$

$\begin{array}{llll}35 & 0.53380100 & -1.62449000 & -2.64207500\end{array}$

$\begin{array}{llll}35 & 0.58530800 & -2.62234800 & 0.58630100\end{array}$

$\begin{array}{llll}7 & -0.20096200 & 0.73891400 & 1.08746000\end{array}$

$\begin{array}{llll}7 & 1.42010800 & 0.88019900 & 1.40119500\end{array}$

$7 \begin{array}{llll}7 & 2.04940700 & 1.30785600 & 2.22055100\end{array}$

$\begin{array}{llll}6 & -0.83356200 & 1.82853200 & 1.70739800\end{array}$

$\begin{array}{llll}6 & -1.19451900 & 2.93979100 & 0.93761300\end{array}$

$\begin{array}{llll}6 & -1.10130300 & 1.78343300 & 3.07978600\end{array}$

$\begin{array}{llll}6 & -1.86598200 & 3.99469600 & 1.54139300\end{array}$

$\begin{array}{llll}6 & -1.76170900 & 2.84948700 & 3.67512200\end{array}$

$\begin{array}{llll}6 & -2.14483100 & 3.95012300 & 2.90711000\end{array}$

$\begin{array}{llll}1 & -0.94783500 & 2.95142300 & -0.12066600\end{array}$

$\begin{array}{llll}1 & -0.80187200 & 0.91164100 & 3.65317600\end{array}$

$\begin{array}{llll}1 & -2.16469600 & 4.85414200 & 0.95094400\end{array}$

$\begin{array}{llll}1 & -1.98541400 & 2.82205900 & 4.73614800\end{array}$

$\begin{array}{llll}1 & -2.66173000 & 4.77977000 & 3.37869500\end{array}$ 
TS8 1

\begin{tabular}{|c|c|c|c|}
\hline & & & \\
\hline 6 & -1.96776900 & -1.34306000 & 0.27775300 \\
\hline 6 & -2.67436400 & -2.09294700 & -0.68886200 \\
\hline 6 & -4.05367900 & -1.95305900 & -0.79067500 \\
\hline 6 & -4.77089600 & -1.10268900 & 0.05755400 \\
\hline 6 & -4.06800200 & -0.38792700 & 1. 02082500 \\
\hline 6 & -2.67915100 & -0.49165500 & 1. 13769200 \\
\hline 6 & 0.15988700 & 1. 40091300 & -0.11402800 \\
\hline 6 & -0.70187600 & 1. 73603200 & -1.18661400 \\
\hline 6 & -1.22844100 & 3. 02506800 & -1.30347200 \\
\hline 6 & -0.94246400 & 4. 02588700 & -0.38351900 \\
\hline 6 & -0.12005500 & 3. 69171700 & 0.68928000 \\
\hline 6 & 0.42594500 & 2. 41530600 & 0.84224100 \\
\hline 5 & -0.40948400 & -1.39375600 & 0.37608900 \\
\hline 5 & 0.69884900 & -0.10466400 & 0.03550100 \\
\hline 17 & 0.32331400 & -2.95644400 & 0.89425200 \\
\hline 17 & 1. 60786000 & -0.68313600 & -1.69047000 \\
\hline 6 & -1.95193600 & -3.03316100 & -1.62440400 \\
\hline 1 & -1.01467500 & -2.60625500 & -2.00034800 \\
\hline 1 & -1.69593500 & -3.97094400 & -1.12096000 \\
\hline 1 & -2.57419500 & -3.27699900 & -2.48909900 \\
\hline 6 & -1.98477700 & 0.30051100 & 2. 22105300 \\
\hline 1 & -0.93670900 & 0.00497600 & 2. 34314200 \\
\hline 1 & -2.00701600 & 1. 37416900 & 2. 00640900 \\
\hline 1 & -2.47330800 & 0.14167000 & 3. 18755800 \\
\hline 1 & -4.60792200 & 0.26846600 & 1. 70113300 \\
\hline 1 & -4.58968900 & -2.51898700 & -1.55057200 \\
\hline 6 & -6.26281700 & -0.96009800 & -0.09096500 \\
\hline 1 & -6.51245200 & -0.38165800 & -0.98699500 \\
\hline 1 & -6.74630500 & -1.93643700 & -0.19035800 \\
\hline 1 & -6.70217800 & -0.44831500 & 0.76858800 \\
\hline 6 & -1.48720400 & 5. 42040100 & -0.54516800 \\
\hline 1 & -1.62314900 & 5. 91008600 & 0.42284800 \\
\hline 1 & -0.80316300 & 6.04133500 & -1.13428500 \\
\hline 1 & -2.44981400 & 5. 41306700 & -1.06339900 \\
\hline 6 & -1.09860700 & 0.76346000 & -2.27498000 \\
\hline 1 & -2.10961900 & 0.98851000 & -2.62601600 \\
\hline 1 & -0.42317500 & 0.83847300 & -3.13445400 \\
\hline 1 & -1.09679100 & -0.27562800 & -1.94809400 \\
\hline 6 & 1. 25085500 & 2. 19641100 & 2. 09313200 \\
\hline 1 & 1. 21760000 & 3. 08860900 & 2. 72271600 \\
\hline 1 & 0.86956900 & 1. 37052700 & 2. 70388900 \\
\hline 1 & 2. 30177300 & 1. 99270200 & 1. 87009100 \\
\hline 1 & -1.88879100 & 3. 24551900 & -2.14057100 \\
\hline 1 & 0.09760700 & 4. 44824000 & 1. 44086000 \\
\hline 14 & 3. 75386700 & -0.46935300 & -0.53569200 \\
\hline 7 & 2. 07342200 & -0.27167700 & 0.79765000 \\
\hline 7 & 2. 10793500 & -0.66283100 & 1. 96298500 \\
\hline 7 & 2. 18838300 & -0.99100800 & 3. 03828100 \\
\hline 6 & 4. 07451500 & 1. 36406500 & -0.37124500 \\
\hline 6 & 4. 53590100 & -1.62548100 & 0.72414700 \\
\hline 6 & 4. 50384900 & -1.06811400 & -2.16261300 \\
\hline 1 & 4. 91453400 & 1. 66696400 & -1.00380800 \\
\hline 1 & 3. 19381300 & 1. 94081200 & -0.67263500 \\
\hline 1 & 4. 30638300 & 1. 62335200 & 0.66630100 \\
\hline 1 & 3. 95924000 & -2.55272600 & 0.82244800 \\
\hline 1 & 5.53485800 & -1.90361000 & 0.37501500 \\
\hline 1 & 4. 63877300 & -1.18412100 & 1. 71974600 \\
\hline 1 & 4. 19290600 & -0.46577900 & -3.02042100 \\
\hline 1 & 5.59563900 & -0.99656300 & -2.07524900 \\
\hline & 4. 25960300 & -2.11442200 & -2.37122100 \\
\hline & & & \\
\hline & -1.96244200 & 0.31939600 & -0.22070500 \\
\hline 6 & -2.96044600 & -0.24835200 & 0.61632500 \\
\hline 6 & -3.79897900 & -1.23966800 & 0.11243600 \\
\hline & -3.71655700 & -1.68212500 & -1.20769700 \\
\hline & -2.73962600 & -1.12214800 & -2.02455600 \\
\hline
\end{tabular}

\begin{tabular}{rrrr}
6 & -1.85609100 & -0.15233500 & -1.55117000 \\
6 & 1.74525900 & 0.25557000 & 0.04256000 \\
6 & 1.73486500 & -0.53776700 & 1.21356500 \\
6 & 2.68193200 & -1.54033500 & 1.38380200 \\
6 & 3.64780200 & -1.80438100 & 0.40743900 \\
6 & 3.63343500 & -1.04037800 & -0.75279800 \\
6 & 2.70215700 & -0.01432900 & -0.95400400 \\
5 & -0.98791500 & 1.43799000 & 0.26473300 \\
5 & 0.73423800 & 1.44432000 & -0.08186000 \\
17 & -1.63013200 & 2.76615700 & 1.26617000 \\
6 & -3.16805300 & 0.14722700 & 2.06139600 \\
1 & -2.23196500 & 0.34499500 & 2.58839400 \\
1 & -3.77753200 & 1.05217100 & 2.14055000 \\
1 & -3.68928900 & -0.65274800 & 2.59283100 \\
6 & -0.80195900 & 0.35870600 & -2.50362700 \\
1 & -0.71638700 & 1.44842900 & -2.49667000 \\
1 & 0.17740200 & -0.05691400 & -2.24151700 \\
1 & -1.02667400 & 0.04623600 & -3.52626800 \\
1 & -2.65409800 & -1.45400500 & -3.05671900 \\
1 & -4.54721600 & -1.67752500 & 0.77039500 \\
6 & -4.65585500 & -2.74113500 & -1.71885900 \\
1 & -4.55257200 & -3.66637300 & -1.14298200 \\
1 & -5.69752200 & -2.41734400 & -1.62875100 \\
1 & -4.46216500 & -2.97249500 & -2.76862100 \\
6 & 4.67526500 & -2.88369000 & 0.62382500 \\
1 & 5.21663400 & -3.11011300 & -0.29774700 \\
1 & 5.40956700 & -2.57714200 & 1.37648800 \\
1 & 4.20977100 & -3.80630900 & 0.98263500 \\
6 & 0.69833800 & -0.30629000 & 2.28582100 \\
1 & -0.29551000 & -0.60486700 & 1.93056300 \\
1 & 0.91605900 & -0.89552800 & 3.17987900 \\
1 & 0.64871900 & 0.74650700 & 2.58515400 \\
6 & 2.77672200 & 0.72658200 & -2.27139700 \\
1 & 2.81629700 & 0.01203700 & -3.09965000 \\
1 & 1.92306400 & 1.38495100 & -2.43997700 \\
1 & 3.68875400 & 1.33127000 & -2.33078300 \\
1 & 2.67115600 & -2.13517000 & 2.29495400 \\
1 & 4.36538000 & -1.24283900 & -1.53276100 \\
7 & 0.83226100 & 2.75099100 & -0.42041900 \\
7 & 2.43897200 & 3.24224300 & -0.74163400 \\
& 3.20744200 & 4.01692300 & -0.89705300 \\
\hline
\end{tabular}
46

\section{TS8_2'}

0. 00639100

$-0.51704500$

$-1.59489200$

$-2.16538100$

$-1.64150700$

$-0.58495300$

0. 18285100

$-0.51253400$

$-1.54636400$

$-1.91584800$

$-1.21533600$

$-0.15893800$

1. 20099100

1. 33559000

2. 64635000

0. 03019700

0. 32757000

0. 91076100

$-0.72393700$

$-0.09586800$

0. 99529700

$-0.43269200$

$-0.49548800$

$-2.06887800$

-0.03420600
0.88250500
0.50554700
-0.76526100
-1.66489800
-1.31544200
0.04991000
1.27891500
1.42900500
0.38881400
-0.80880900
-0.99438700
0.29243300
-0.04598200
1.10686500
2.27888100
2.75167800
2.27259600
2.91474100
-2.34256700
-2.42564800
-2.08578800
-3.33171500
-2.66229800

$-3.4635550$

$1-0.74979600$

$1-1.00344300$

$1-2.52482000$

\section{.}

. 


\begin{tabular}{|c|c|c|c|}
\hline 1 & -4.31164000 & -1.99991100 & 1. 22356400 \\
\hline 6 & -4.41134000 & -3.32266700 & -1.13757000 \\
\hline 1 & -4.14087500 & -4.21884400 & -0.56955200 \\
\hline 1 & -5.45951400 & -3.10141900 & -0.91524500 \\
\hline 1 & -4.33088700 & -3.56082200 & -2.20062700 \\
\hline 6 & 4. 70108500 & -3.05324200 & 0.57194400 \\
\hline 1 & 5. 44223800 & -3.07855400 & -0.23029500 \\
\hline 1 & 5. 23127400 & -2.96834700 & 1. 52503300 \\
\hline 1 & 4. 17761100 & -4.01538100 & 0.57604500 \\
\hline 6 & 1. 02939000 & -0.16049100 & 2. 41415000 \\
\hline 1 & 0.00135700 & -0.46265000 & 2. 17415800 \\
\hline 1 & 1. 31268800 & -0.67869200 & 3. 33339400 \\
\hline 1 & 1. 02704200 & 0.91512400 & 2. 62104300 \\
\hline 6 & 2. 79882500 & 0.57764800 & -2.31263600 \\
\hline 1 & 3. 03623300 & -0.11049800 & -3.12900500 \\
\hline 1 & 1. 86091400 & 1. 08422800 & -2.54175400 \\
\hline 1 & 3. 57608500 & 1. 34873400 & -2.29089000 \\
\hline 1 & 2. 92254800 & -2.08114400 & 2. 37562900 \\
\hline 1 & 4. 32334300 & -1.48312500 & -1.62491700 \\
\hline 7 & 1. 58254100 & 2. 38663300 & -0.26879900 \\
\hline 7 & 0.72341200 & 3. 92511800 & -0.40623800 \\
\hline 7 & 0.75751200 & 5. 01925100 & -0.52860600 \\
\hline \multicolumn{4}{|c|}{60} \\
\hline \multicolumn{4}{|c|}{ TS8_3 } \\
\hline 6 & 3. 15891700 & -0.00092300 & 0.35481700 \\
\hline 6 & 3. 26359500 & 0.58957900 & -0.91735300 \\
\hline 6 & 4. 25997100 & 0.16460300 & -1.79650200 \\
\hline 6 & 5. 15411400 & -0.84806600 & -1.45091100 \\
\hline 6 & 5. 03324400 & -1.43443900 & -0.19138000 \\
\hline 6 & 4. 05601700 & -1.02217800 & 0.71469300 \\
\hline 6 & -1.68701600 & -1.19835700 & 0.48327700 \\
\hline 6 & -1.63178000 & -2.06387800 & -0.62857100 \\
\hline 6 & -2.71116500 & -2.90482400 & -0.88523000 \\
\hline 6 & -3.84247200 & -2.91156600 & -0.06338200 \\
\hline 6 & -3.88057300 & -2.04757800 & 1. 03140700 \\
\hline 6 & -2.81646200 & -1.19218400 & 1. 32040900 \\
\hline 5 & 1. 99906100 & 0.41715000 & 1. 33268500 \\
\hline 5 & -0.49256900 & -0.28302400 & 0.76521900 \\
\hline 17 & 2. 43375800 & 1. 33089600 & 2. 83950500 \\
\hline 6 & 2. 30995200 & 1.68357800 & -1.33336200 \\
\hline 1 & 1. 27644000 & 1. 31723500 & -1.36410900 \\
\hline 1 & 2. 33801600 & 2. 52063700 & -0.62717400 \\
\hline 1 & 2. 56234500 & 2. 07056600 & -2.32371100 \\
\hline 6 & 3. 96989600 & -1.68011800 & 2. 07100100 \\
\hline 1 & 4. 36552200 & -1.02390500 & 2. 85317300 \\
\hline 1 & 2.93440200 & -1.91511800 & 2. 34003700 \\
\hline 1 & 4. 54118900 & -2.61159000 & 2. 09334900 \\
\hline 1 & 5.71459600 & -2.23510000 & 0.09075800 \\
\hline 1 & 4. 33815400 & 0.63058000 & -2.77709500 \\
\hline 6 & 6.23714200 & -1.28331900 & -2.40364100 \\
\hline 1 & 6. 43298700 & -2.35588500 & -2.32030200 \\
\hline 1 & 5.96662000 & -1.06491100 & -3.44007900 \\
\hline 1 & 7. 17763900 & -0.76215400 & -2.19308900 \\
\hline 6 & -4.98155500 & -3.85318100 & -0.35013200 \\
\hline 1 & -5.87131700 & -3.59184000 & 0.22720100 \\
\hline 1 & -5.24521600 & -3.84353400 & -1.41163200 \\
\hline 1 & -4.70579300 & -4.88152500 & -0.09321200 \\
\hline 6 & -0.44107300 & -2.04333000 & -1.55272500 \\
\hline 1 & -0.49221900 & -2.85447800 & -2.28254700 \\
\hline 1 & -0.39793300 & -1.09739500 & -2.10583000 \\
\hline 1 & 0.50006000 & -2.13374800 & -1.00130400 \\
\hline 6 & -2.86805700 & -0.28383500 & 2. 52196600 \\
\hline 1 & -2.22495000 & -0.66379500 & 3. 32325600 \\
\hline 1 & -2.51240600 & 0.72175300 & 2. 27287700 \\
\hline 1 & -3.88345900 & -0.20221900 & 2. 91744400 \\
\hline 1 & -2.67472900 & -3.57042300 & -1.74504800 \\
\hline 1 & -4.75645500 & -2.04312000 & 1. 67663800 \\
\hline 7 & 0.67522500 & 0.10991500 & 1. 10144700 \\
\hline
\end{tabular}

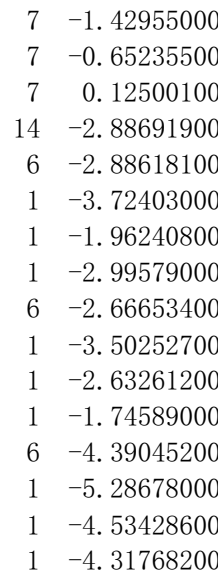

88

TS8_3-Di

$6-3.25122000$

$\begin{array}{ll}6 & -3.23778800\end{array}$

$6-4.37096700$

$\begin{array}{ll}6 & -5.52810600\end{array}$

$\begin{array}{ll}6 & -5.53497300\end{array}$

$\begin{array}{ll}6 & -4.42114400\end{array}$

62.03282400

63.01194500

64.33632900

6
6

$6 \quad 3.71471400$

$6 \quad 2.38417300$

$\begin{array}{ll}5 & -1.94814400\end{array}$

$5 \quad 0.57669200$

$\begin{array}{ll}17 & -2.01887000\end{array}$

$\begin{array}{ll}6 & -2.01944200\end{array}$

$1-1.17333100$

$1-1.68462900$

$1-2.24256700$

$\begin{array}{ll}6 & -4.52009400\end{array}$

$1-4.78446700$

$1-3.58070600$

$1-5.29059300$

$1-6.42873000$

$1-4.35150600$

$6-6.72564600$

$1-6.46211900$

$1-7.11934500$

$1-7.53023400$

$6 \quad 6.13825300$

16.28649600

16.43226500

$1 \quad 6.81200300$

$6 \quad 2.64896300$

12.29893300

13.51366800

$1 \quad 1.86083500$

$6 \quad 1.33633900$

11.74844200

10.48455000

10.95523000

15.10046500

$1 \quad 3.99652300$

$\begin{array}{ll}7 & -0.68254100\end{array}$

$6 \quad 3.06498700$

$6 \quad 4.28325700$

65.41009000

$\begin{array}{ll}6 & 5.37616500\end{array}$

64.17078600

$6 \quad 3.01867400$
1. 65803300

2. 54294400

3. 27444000

2. 04049100

0. 83334100

1. 04706800

0. 90902300

$-0.19702500$

3. 80794000

4. 10662400

4. 50871000

3. 92994700

1. 84265600

2. 16178800

0. 79763500

2. 44951900

1. 44026300

1. 28919200

0. 80944500

0. 46518600

0. 63684300

1. 12638500

1. 96242600

1. 23771100

1. 66704300

2. 78854300

3. 49748500

3. 10592900

1. 92827200

1. 57976800

3. 44207100

1. 65467500

0. 98659100

2. 67479600

1. 60416000

1. 30061000

2. 33214400

1. 06780700

0. 64276100

0. 37805000

0. 69410500

$-0.08796700$

$-0.99252400$

0. 63378000

$-0.34154800$

3. 23226500

4. 15998900

3. 43145600

2. 47716700

0.02709300

0. 32206100

$-0.63122200$

$-0.55317700$

3. 85999700

4. 77729400

4. 13633900

3. 24931300

1. 10006900

4. 36745700

1. 30569500

$-1.74336000$

$-1.05350000$

$-1.46995200$

$-2.57818500$

-3. 26600700

$-2.86247600$
$-0.00100500$

0. 34662000

0. 71018700

$-0.99321800$

-2. 41219500

$-3.08513600$

$-2.99411800$

$-2.05910500$

$-1.56520800$

$-2.20629400$

$-0.72440500$

$-2.14545400$

0. 09374700

$-0.45004900$

0. 38577400

1. 00131700

0. 07545600

1. 48030400

2. 13103300

1. 42828000

0. 04773200

$-0.63865600$

$-0.52137000$

$-1.22775200$

$-1.16208100$

-0. 41994700

0. 27462900

0. 23721500

$-0.63466800$

$-0.55394500$

$-1.62691300$

2. 29823400

2. 10068300

2. 07757000

3. 36681600

$-2.13524800$

$-2.39239200$

$-2.64386000$

$-2.54711100$

$-0.51707400$

3. 21341500

2. 15465300

2. 71284000

2. 87760200

1. 45989600

$-0.34607500$

$-0.90908200$

0. 68859700

$-0.75696500$

$-2.04445900$

$-3.04061300$

$-2.16469800$

$-1.55460300$

1. 01539100

1. 44053500

0. 38394600

1. 84151500

$-1.68827600$

0. 86319300

$-0.51287800$

0. 77048700

0.92685600

0. 21699100

$-0.63006300$

$-0.76318800$

$-0.08591000$ 


$\begin{array}{rr}6 & -1.83544700 \\ 6 & -2.17631200 \\ 6 & -3.42071600 \\ 6 & -4.33433600 \\ 6 & -3.96455900 \\ 6 & -2.73030900 \\ 5 & 1.73519800 \\ 5 & -0.51291500 \\ 17 & 1.66764000 \\ 6 & 4.39710100 \\ 1 & 3.57418700 \\ 1 & 4.37551400 \\ 1 & 5.33444100 \\ 6 & 1.73796200 \\ 1 & 1.17215500 \\ 1 & 1.07633500 \\ 1 & 1.94946000 \\ 1 & 4.12516600 \\ 1 & 6.34128700 \\ 6 & 6.61721800 \\ 1 & 7.23989000 \\ 1 & 7.22824300 \\ 1 & 6.36898300 \\ 6 & -5.69824200 \\ 1 & -5.86649900 \\ 1 & -5.82815100 \\ 1 & -6.47925200 \\ 6 & -1.24705000 \\ 1 & -0.19695000 \\ 1 & -1.38294600 \\ 1 & -1.43193900 \\ 6 & -2.36253300 \\ 1 & -3.23013700 \\ 1 & -1.57588100 \\ 1 & -1.98205500 \\ 1 & -3.68544900 \\ 1 & -4.66610700 \\ 7 & 0.59581600 \\ 60 & \end{array}$

$-1.38474900$

$-1.36250000$

$-2.81660100$

60

TS8_4

$6 \quad 1.73623000$

$6 \quad 1.37953300$

$6 \quad 1.33899000$

$\begin{array}{ll}6 & 1.64088700\end{array}$

$\begin{array}{ll}6 & 1.99296800\end{array}$

$\begin{array}{ll}6 & 2.04855600\end{array}$

$6-1.47467600$

$\begin{array}{ll}6 & -1.37528600\end{array}$

$\begin{array}{ll}6 & -2.19407500\end{array}$

$\begin{array}{ll}6 & -3.10438800\end{array}$

$\begin{array}{ll}6 & -3.17511400\end{array}$

$\begin{array}{ll}6 & -2.36791900\end{array}$

$\begin{array}{ll}5 & 1.75342300\end{array}$

$5-0.56747000$

$17 \quad 3.39830700$

$6 \quad 1.06687700$

$1 \quad 0.27789400$

$1 \quad 1.94950100$

$1 \quad 0.74180900$

$\begin{array}{ll}6 & 2.45753700\end{array}$

13.44787800

$1 \quad 1.76017100$

12.49451700

12.23027200

$1 \quad 1.06685300$

$6 \quad 1.59426500$

11.34733200

$1 \quad 2.55843000$
$-1.85402500$

$-2.35801600$

$-2.41125500$

$-1.94466500$

$-1.25172200$

$-0.81209300$

$-1.15637000$

0.14267800

0. 85010600

$-0.16358200$

0. 67728700

$-3.63087000$

$-3.77632000$

$-3.10104200$

$-4.61472900$

$-4.13638700$

$-0.91693400$

$-3.03737500$

$-2.18985400$

$-3.67776700$

$-3.61379200$

$-2.82931000$

$-2.65732800$

$-3.89859000$

$-2.30189700$

$-0.79506100$

$-0.89694800$

$-1.30745800$

0. 27265600

$-2.05665300$

$-2.35289400$

$-2.80523600$

$-1.11371400$

$-1.82770400$

$-0.92472900$

$-0.41946500$

$-1.78193700$

-2. 18240800

$-1.26133200$

0. 08529900

0. 52192800

1. 45501900

0. 11563700

3. 27641100

1. 84037500

1. 68813500

2. 89089100

1. 66255300

$-0.31642900$

0.60855900

$-1.01205200$

$-0.74278900$

$-1.41522600$

0.33194700

$-1.34996200$

$-1.65000400$

$-0.70433500$

$-2.24478600$

$-1.68804200$

$-2.75389000$

$-1.49192700$

$-1.12974700$

$-2.82641100$

-2. 53640900

$-3.78263600$

$-2.99794500$

1. 98016300

2. 57492100

2. 12751000

2. 38311400

$-3.23784900$

0. 81232900

0. 76291900

$-1.41385500$

$-2.77278100$

$-3.50579900$

$-2.91937300$

$-1.56830100$

$-0.81321900$

$-1.19129700$

$-1.46169200$

$-2.43620000$

$-3.16356300$

$-2.90282700$

$-1.93299300$

$-0.58907000$

$-0.09032400$

$-0.23907800$

$-3.44868500$

$-2.92251100$

$-3.46970300$

$-4.48110400$

0.63817700

0.78381300

1. 27310800

1. 00104900

$-1.09383800$

$-4.55905500$

$-3.71305800$

$-4.76022700$

$-3.68275500$
2. 88158500

2. 84967400

4. 03871100

5. 26584000

5. 28465800

4. 11450200

$-0.36014700$

$-1.73612200$

$-2.30527500$

$-1.53503100$

$-0.16666300$

0. 43158600

1. 53897000

0. 30573800

0. 81397700

1. 53588600

0. 98668800

0. 88610500

1. 68818300

4. 17070300

3. 72603100

3. 61314900

5. 20094300

6. 23572700

4. 00411400

6. 54614400

6. 35543100

7. 06362200
10.84501400

$6-3.97656300$

$1-4.67874800$

$1-4.55439000$

$1-3.37249900$

$6-0.36544700$

$1-0.63424800$

$1-0.26805900$

0. 62680700

$6-2.42968600$

$1-1.52127100$

$1-2.49773500$

$1-3.28895500$

$1-2.11913600$

$1-3.87099800$

$7 \quad 0.63664000$

$7-1.20548800$

$7 \quad-0.36332700$

$7 \quad 0.67101900$

$14 \quad-2.79949800$

$\begin{array}{ll}6 & -2.89705700\end{array}$

$1 \quad-3.80497900$

$1-2.04435700$

$1-2.95553000$

$6-2.69295400$

$1-3.60624400$

$1-2.59245200$

$1-1.85046900$

$6-4.17558300$

$1-5.13495900$

$1-4.19849800$

$1-4.09453900$

120

TS8_5

$6 \quad 2.34964300$

63.14294900

64.00073900

64.12204400

63.36257100

$6 \quad 2.49365500$

64.42912100

65.00843500

$6 \quad 6.24265400$

$6 \quad 6.94137300$

$6 \quad 6.37880400$

65.13350100

$5 \quad 1.41218500$

$5 \quad 2.99389400$

$17 \quad 0.46519400$

63.16035200

13.12871300

12.30919800

14.07650000

$6 \quad 1.73852600$

$1 \quad 1.51652800$

$1 \quad 2.31643000$

$1 \quad 0.78191400$

13.45200000

14.60919900

65.08922200

16.11617500

15.05682400

14.87169400

$6 \quad 8.25857600$

18.11304300

18.75574000

18.93582500

$6 \quad 4.32118700$

$-3.31037400$

$-4.21181100$

$-4.63946700$

$-3.79189100$

$-5.02883200$

$-0.72868400$

$-0.75758500$

0. 31986900

$-1.18021000$

$-1.69566400$

$-2.06738400$

$-0.62760400$

$-2.19718000$

$-2.63815500$

$-3.46958600$

$-0.12851800$

1. 35365200

2. 15567400

2. 14318800

2. 00767400

1. 68601300

2. 15938200

2. 11409000

0. 61763900

3. 83172800

4. 33301000

4. 01911900

4. 30910600

1. 17002800

1. 58667200

0. 09367300

1. 33289100

$-1.73079600$

$-2.52953500$

$-3.49210100$

$-3.70244600$

$-2.90122400$

$-1.92106400$

0. 84380000

0. 64239300

0. 00963800

$-0.41790600$

$-0.17921600$

0. 44380300

$-0.59334000$

1. 46910100

$-0.79828900$

$-2.37836100$

$-1.33425600$

$-2.88206100$

$-2.81794200$

$-1.12846500$

$-0.11044000$

$-1.08483400$

$-1.60607300$

$-3.03091700$

$-4.08396400$

$-4.72084900$

$-4.34161600$ 


\begin{tabular}{|c|c|c|}
\hline 3. 24603900 & 0.94555500 & 2. 69289100 \\
\hline 4. 75312800 & 0.71392500 & 3. 59403300 \\
\hline 4. 42860300 & 2. 24429900 & 2. 77253200 \\
\hline 4. 59181100 & 0.68076400 & -2.35398200 \\
\hline 4.50633800 & -0.26237300 & -2.90337100 \\
\hline 3. 60338500 & 1. 15043400 & -2.33620900 \\
\hline 5. 26134100 & 1. 33076100 & -2.92880900 \\
\hline 6.67690800 & -0.15244500 & 2. 53695300 \\
\hline 6.91278000 & -0.49296100 & -1.72631600 \\
\hline 1. 72981400 & 0.80620300 & 0.35076300 \\
\hline 2.59338300 & 2.84415700 & 0.13744900 \\
\hline 1. 23888300 & 2. 90875000 & 0.36631000 \\
\hline 0.75654800 & 1. 75981400 & 0.51018500 \\
\hline 3. 39694200 & 4. 42217200 & -0.13080400 \\
\hline 5. 21439600 & 4. 06609300 & -0.37220600 \\
\hline 5. 65556000 & 3.55807000 & 0.49064200 \\
\hline 5. 38685300 & 3. 43801700 & -1.25176100 \\
\hline 5. 75675500 & 5. 00634700 & -0.52242700 \\
\hline 3. 07306300 & 5. 47769100 & 1. 37688000 \\
\hline 3.50195700 & 6.47814600 & 1. 25648900 \\
\hline 1. 99749800 & 5.59009200 & 1. 54450100 \\
\hline 3.50780400 & 5. 03180200 & 2. 27695400 \\
\hline 2. 62151200 & 5. 16398800 & -1.66131000 \\
\hline 3. 02124700 & 6. 16164000 & -1.87076300 \\
\hline 2. 80830300 & 4.53293600 & -2.53641200 \\
\hline 1. 53734800 & 5. 25020800 & -1.53776000 \\
\hline-2.99463500 & 2. 03440600 & -0.43700500 \\
\hline-3.08363100 & 2. 66333800 & 0.82102400 \\
\hline-4.25469200 & 3. 33782200 & 1. 16710100 \\
\hline-5.35124900 & 3. 39670300 & 0.30807900 \\
\hline-5.24164700 & 2. 79024200 & -0.94128300 \\
\hline-4.08072000 & 2. 12395800 & -1.33009100 \\
\hline-4.22958200 & -0.89303500 & 0.08484600 \\
\hline-4.77198000 & -0.32353200 & 1. 24874400 \\
\hline-6.12824200 & 0.00351300 & 1. 28925100 \\
\hline-6.96600000 & -0.22308700 & 0. 19968900 \\
\hline-6.42628000 & -0.83316200 & -0.93460600 \\
\hline-5.07848000 & -1.18079000 & -1.00319800 \\
\hline-1.76348600 & 1. 16120600 & -0.84684800 \\
\hline-2.69507600 & -1.14181200 & -0.05442200 \\
\hline-0.69507900 & 1. 67411300 & -2.17867600 \\
\hline-1.93121200 & 2. 64931400 & 1. 79216800 \\
\hline-1.46531900 & 1. 66297500 & 1. 86972000 \\
\hline
\end{tabular}
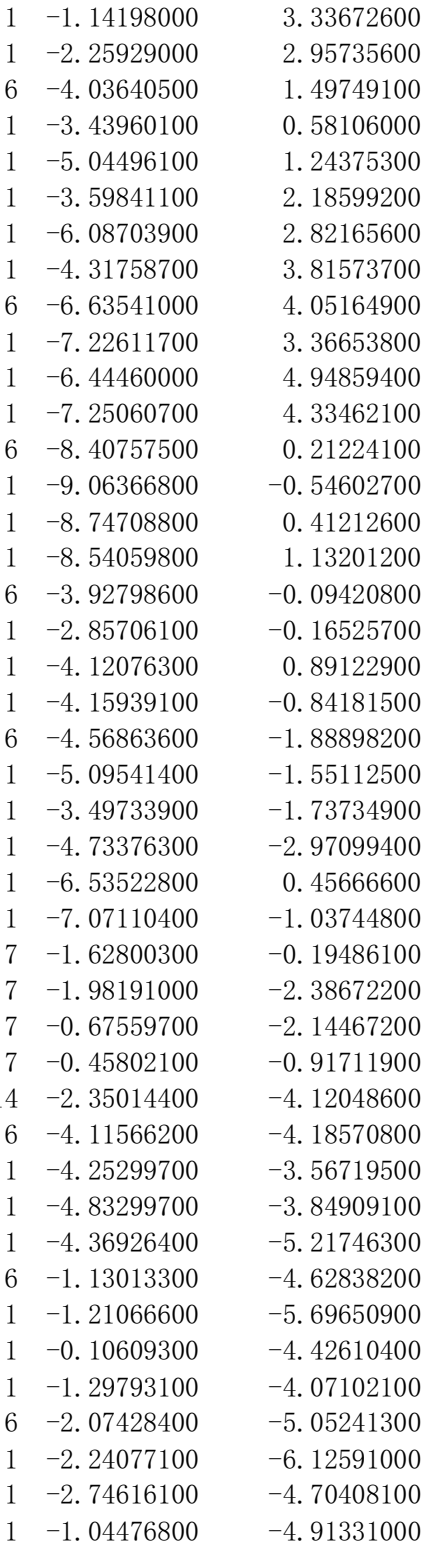

\section{References}

(1) Kwok, S. W.; Fotsing, J. R.; Fraser, R. J.; Rodionov, V. O.; Fokin, V. V., Transition-MetalFree Catalytic Synthesis of 1,5-Diaryl-1,2,3-triazoles. Org. Lett. 2010, 12, 4217-4219.

(2) Hunold, R. Verbindungen mit partieller Bor-Kohlenstoff-Dreifachbindung und ein Methylenboran hoher Lewis-Acidität, Doctoral Thesis, Philipps-Universität Marburg, Marburg, Germany, 1988.

(3) Hunold, R.; Allwohn, J.; Baum, G.; Massa, W.; Berndt, A., Verbindungen mit partieller BorKohlenstoff-Dreifachbindung. Angew. Chem. 1988, 100, 961-963.

(4) Braunschweig, H.; Damme, A.; Dewhurst, R. D.; Vargas, A., Bond-strengthening $\pi$ backdonation in a transition-metal $\pi$-diborene complex. Nat. Chem. 2012, 5, 115-121.

(5) Wang, S. R.; Arrowsmith, M.; Böhnke, J.; Braunschweig, H.; Dellermann, T.; Dewhurst, R. D.; Kelch, H.; Krummenacher, I.; Mattock, J. D.; Müssig, J. H.; Thiess, T.; Vargas, A.; Zhang, J., Engineering a Small HOMO-LUMO Gap and Intramolecular $\mathrm{C}-\mathrm{H}$ Borylation by Diborene/Anthracene Orbital Intercalation. Angew. Chem. Int. Ed. 2017, 56, 8009-8013.

(6) Müssig, J. H.; Prieschl, D.; Deißenberger, A.; Dewhurst, R. D.; Dietz, M.; Jiménez-Halla, J. O. C.; Trumpp, A.; Wang, S. R.; Brunecker, C.; Haefner, A.; Gärtner, A.; Thiess, T.; Böhnke, J.; Radacki, K.; Bertermann, R.; Marder, T. B.; Braunschweig, H., Reactivity of Tetrahalo- and 
Difluorodiboranes(4) toward Lewis Basic Platinum(0): Bis(boryl), Borylborato, and Doubly BorylBridged Platinum Complexes. J. Am. Chem. Soc. 2018, 140, 13056-13063.

(7) Sheldrick, G., SHELXT - Integrated space-group and crystal-structure determination. Acta Crystallogr. Sect. A: Found. Crystallogr. 2015, 71, 3-8.

(8) Sheldrick, G., A short history of SHELX. Acta Crystallogr. Sect. A: Found. Crystallogr. 2008, $64,112-122$.

(9) Zhao, Y.; Truhlar, D. G., The M06 suite of density functionals for main group thermochemistry, thermochemical kinetics, noncovalent interactions, excited states, and transition elements: two new functionals and systematic testing of four M06-class functionals and 12 other functionals. Theor. Chem. Acc. 2008, 120, 215-241.

(10) Marenich, A. V.; Cramer, C. J.; Truhlar, D. G., Universal Solvation Model Based on Solute Electron Density and on a Continuum Model of the Solvent Defined by the Bulk Dielectric Constant and Atomic Surface Tensions. J. Phys. Chem. B 2009, 113, 6378-6396.

(11) Hay, P. J.; Wadt, W. R., Ab initio effective core potentials for molecular calculations. Potentials for the transition metal atoms Sc to Hg. J. Chem. Phys. 1985, 82, 270-283.

(12) Hay, P. J.; Wadt, W. R., Ab initio effective core potentials for molecular calculations. Potentials for K to Au including the outermost core orbitals. J. Chem. Phys. 1985, 82, 299-310.

(13) Höllwarth, A.; Böhme, M.; Dapprich, S.; Ehlers, A. W.; Gobbi, A.; Jonas, V.; Köhler, K. F.; Stegmann, R.; Veldkamp, A.; Frenking, G., A set of d-polarization functions for pseudo-potential basis sets of the main group elements $\mathrm{Al}$ - $\mathrm{Bi}$ and f-type polarization functions for $\mathrm{Zn}, \mathrm{Cd}, \mathrm{Hg}$. Chem. Phys. Lett. 1993, 208, 237-240.

(14) Fukui, K., Formulation of the reaction coordinate. J. Phys. Chem. 1970, 74, 4161-4163.

(15) Fukui, K., The path of chemical reactions - the IRC approach. Acc. Chem. Res. 1981, 14, 363368.

(16) Frisch, M. J.; Trucks, G. W.; Schlegel, H. B.; Scuseria, G. E.; Robb, M. A.; Cheeseman, J. R.; Scalmani, G.; Barone, V.; Mennucci, B.; Petersson, G. A.; Nakatsuji, H.; Caricato, M.; Li, X.; Hratchian, H. P.; Izmaylov, A. F.; Bloino, J.; Zheng, G.; Sonnenberg, J. L.; Hada, M.; Ehara, M.; Toyota, K.; Fukuda, R.; Hasegawa, J.; Ishida, M.; Nakajima, T.; Honda, Y.; Kitao, O.; Nakai, H.; Vreven, T.; Montgomery, J. J. A.; Peralta, J. E.; Ogliaro, F.; Bearpark, M.; Heyd, J. J.; Brothers, E.; Kudin, K. N.; Staroverov, V. N. K., R.; Normand, J.; Raghavachari, K.; Rendell, A.; Burant, J. C.; Iyengar, S. S.; Tomasi, J.; Cossi, M.; Rega, N.; Millam, N. J.; Klene, M.; Knox, J. E.; Cross, J. B.; Bakken, V.; Adamo, C.; Jaramillo, J.; Gomperts, R.; Stratmann, R. E.; Yazyev, O.; Austin, A. J.; Cammi, R.; Pomelli, C.; Ochterski, J. W.; Martin, R. L.; Morokuma, K.; Zakrzewski, V. G.; Voth, G. A.; Salvador, P.; Dannenberg, J. J.; Dapprich, S.; Daniels, A. D.; Farkas, Ö.; Foresman, J. B.; Ortiz, J. V.; Cioslowski, J.; Fox, D. J. Gaussian 09, Revision A.02; Gaussian, Inc.: Wallingford CT, 2009. 